\title{
Near-Capacity Variable Length Coding
}

L. Hanzo, R. G. Maunder, J. Wang. L-L. Yang

March 10, 2010 


\section{Contents}

Chapter 1 Information Theory Basics 11

1.1 Issues in Information Theory . . . . . . . . . . . . . . . . . . 11

1.2 Additive White Gaussian Noise Channel . . . . . . . . . . . . . . . . . . 15

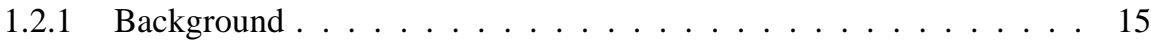

1.2.2 Practical Gaussian Channels . . . . . . . . . . . . . . 15

1.2 .3 Gaussian Noise . . . . . . . . . . . . . . . . . . . . . . 16

1.3 Information of a Source . . . . . . . . . . . . . . . . 18

1.4 Average Information of Discrete Memoryless Sources . . . . . . . . . . . . . 20

1.4.1 Maximum Entropy of a Binary Source . . . . . . . . . . . . . . . . 21

1.4.2 Maximum Entropy of a $q$-ary Source . . . . . . . . . . . . . . . 22

1.5 Source Coding for a Discrete Memoryless Source . . . . . . . . . . . . . 23

1.5.1 Shannon-Fano Coding .................. 24

1.5.2 Huffman Coding . . . . . . . . . . . . . . . . . . . 26

1.6 Entropy of Discrete Sources Exhibiting Memory _ . . . . . . . . . . . 30

1.6.1 Two-State Markov Model for Discrete Sources Exhibiting Memory . 30

1.6.2 N-State Markov Model for Discrete Sources Exhibiting Memory . . 31

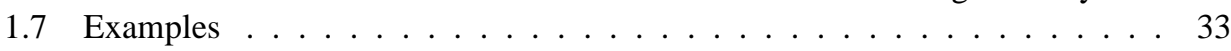

1.7.1 Two-State Markov Model Example . . . . . . . . . . . . . . . . 33

1.7.2 Four-State Markov Model for a 2-Bit Quantizer . . . . . . . . . . . . 35

1.8 Generating Model Sources . . . . . . . . . . . . . . . . . 36

1.8.1 Autoregressive Model . . . . . . . . . . . . . . . . 36

1.8.2 AR Model Properties . . . . . . . . . . . . . . . . . . . . . . . . . . . . . . . . 37

1.8.3 First-Order Markov Model . . . . . . . . . . . . . . . . . . . 37

1.9 Run-Length Coding for Discrete Sources Exhibiting Memory . . . . . . . . . 39

1.9.1 Run-Length Coding Principle . . . . . . . . . . . . . . . . . . . 39

1.9.2 Run-Length Coding Compression Ratio . . . . . . . . . . . . . . . . 40

1.10 Information Transmission via Discrete Channels . . . . . . . . . . . . . . . . 42

1.10.1 Binary Symmetric Channel Example . . . . . . . . . . . . . . . . 42

1.10 .2 Bayes' Rule . . . . . . . . . . . . . . . . . . . . . . . . . . . . . . . . . . . . 44

1.10 .3 Mutual Information . . . . . . . . . . . . . . . . . . 47 
1.10.4 Mutual Information Example . . . . . . . . . . . . . . . . . . . 48

1.10.5 Information Loss via Imperfect Channels . . . . . . . . . . . . . 50

1.10.6 Error Entropy via Imperfect Channels . . . . . . . . . . . . . . . 52

1.11 Capacity of Discrete Channels . . . . . . . . . . . . . . . . . . 59

1.12 Shannon's Channel Coding Theorem . . . . . . . . . . . . . . . . . 61

1.13 Capacity of Continuous Channels . . . . . . . . . . . . . . . . . 64

1.13.1 Practical Evaluation of the Shannon-Hartley Law . . . . . . . . . . 68

1.13.2 Shannon's Ideal Communications System for Gaussian Channels . . . 71

1.14 Shannon's Message for Wireless Channels . . . . . . . . . . . . . . . . . . 72

1.15 Summary and Conclusions . . . . . . . . . . . . . . . . . . . . . . . . . . . . . 74

1.16 Structure and novel aspects of the book . . . . . . . . . . . . 75

Chapter 4 Ingredients of Irregular Variable Length Codes 130

4.1 Applications of irregular variable length coding . . . . . . . . . . . . . . 130

4.1.1 Near-capacity operation . . . . . . . . . . . . . . . . . 130

4.1.2 Joint source and channel coding . . . . . . . . . . . . . . . . . . 132

4.1.3 Unequal error protection . . . . . . . . . . . . . . . . . . 133

4.2 Variable length coding revisited . . . . . . . . . . . . . . . . . . . . . . . . . . . . . . . . . . .

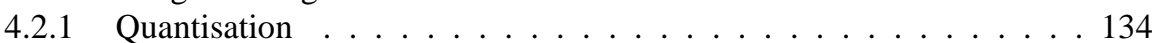

4.2 .2 Entropy . . . . . . . . . . . . . . . . . . 135

4.2.3 Shannon-Fano coding . . . . . . . . . . . . . . 136

4.2.4 Huffman coding . . . . . . . . . . . . . . . . . . 137

4.2.5 Reversible variable length coding . . . . . . . . . . . . . . . . 138

4.2.6 Variable length error correction coding . . . . . . . . . . . . . 140

4.2.6.1 Bit-based VLEC trellis . . . . . . . . . . . . . . . 140

4.2.6.2 ML VLEC sequence estimation . . . . . . . . . . . . . . 142

4.2.6.3 Symbol-based VLEC trellis . . . . . . . . . . . . . 143

4.2.6.4 VLEC error correction capability . . . . . . . . . . . . 145

4.3 The turbo principle . . . . . . . . . . . . . . . . . . . 147

4.3.1 Hard and soft information . . . . . . . . . . . . . . . . . 147

4.3.2 Soft VLEC decoders . . . . . . . . . . . . . . . . . . . . . . 148

4.3.2.1 ML SIHO VLEC sequence estimation . . . . . . . . . . . 148

4.3.2.2 SISO VLEC algorithms . . . . . . . . . . . . . . . 149

4.3.2.3 MAP SIHO VLEC sequence estimation . . . . . . . . . 151

4.3.2.4 Computational complexity and other implementational issues 152

4.3.3 Concatenated codes . . . . . . . . . . . . . . . . . . 154

4.3.3.1 Parallel concatenation . . . . . . . . . . . . . . 154

4.3.3.2 Serial concatenation . . . . . . . . . . . . . 156

4.3.4 Iterative decoding convergence . . . . . . . . . . . . . . . . . 158

4.3.4.1 Mutual information . . . . . . . . . . . . . . . . . . . . . . . . . . . . . . . . .

4.3.4.2 EXIT function . . . . . . . . . . . . . . . . . . . . . . . . . . . . . . . .

4.3.4.3 EXIT chart ... . . . . . . . . . . . 162

4.3.4.4 Area properties of EXIT charts . . . . . . . . . . 167

4.4 Irregular coding . . . . . . . . . . . . . . . . . . 169 
6.1 Introduction . . . . . . . . . . . . . . . . . . 228

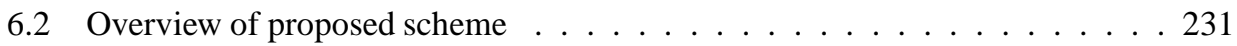

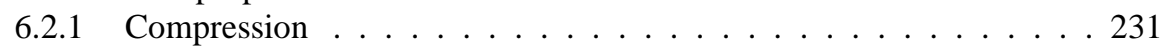

6.2.2 VDVQ/RVLC decomposition ................... 234

6.2.3 Serial concatenation and iterative decoding . . . . . . . . . . . 234

6.3 Transmission frame structure . . . . . . . . . . . . . . . 235

6.3.1 Frame difference decomposition . . . . . . . . . . . . 236

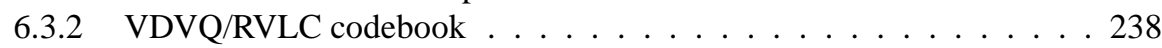

6.3.3 VDVQ/RVLC-induced code constraints . . . . . . . . . . . . 239

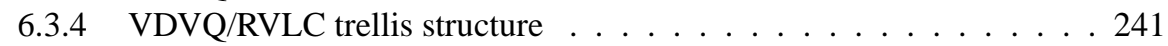

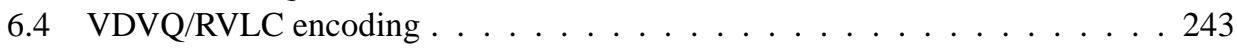

6.5 APP SISO VDVQ/RVLC decoding . . . . . . . . . . . . . . . . . . . . . . . . . . . . . . . . . . . . . .

6.6 Simulation results . . . . . . . . . . . . . . . . . . . . . . . . . . . . . . . . . . . . . . . . . . . . .

6.7 Summary and Conclusions . . . . . . . . . . . . . . . 253

Chapter 7 Irregular Variable Length Codes for EXIT Chart Matching 255

7.1 Introduction . . . . . . . . . . . . . . . . 255

7.2 Overview of proposed schemes . . . . . . . . . . . . . . . . . . . . . . . . . . . . . 58

7.2.1 Joint source and channel coding . . . . . . . . . . . . . . . 259

7.2 .2 Iterative decoding . . . . . . . . . . . . . . . . . . . . . . . 262

7.3 Parameter design for the proposed schemes . . . . . . . . . . . . . . . 264

7.3.1 Scheme hypothesis and parameters . . . . . . . . . . . . . 264

7.3.2 EXIT chart analysis and optimisation . . . . . . . . . . . . . . . . . . . . . . . . . . . . . . . . . . .

7.4 Simulation results . . . . . . . . . . . . . . . . . . . . . . . . . . . . . . . . . . . . . . .

7.4.1 IrCC-based benchmarker . . . . . . . . . . . . . . . 272

7.4.2 Iterative decoding convergence performance . . . . . . . . . . . 272

7.4.3 Interleaver length and latency . . . . . . . . . . . . . . . . 276

7.4.4 Performance during iterative decoding . . . . . . . . . . . . . . . . 277

7.4 .5 Complexity analysis . . . . . . . . . . . . . . . . 278

7.4.6 Unequal error protection performance . . . . . . . . . . . 281

7.5 Summary and Conclusions . . . . . . . . . . . . . . . . 283

Chapter 8 Genetic Algorithm Aided Design of Irregular Variable Length Coding Components 286

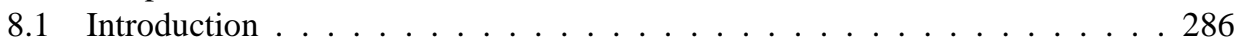

8.2 The free distance metric . . . . . . . . . . . . . . . . . . . . 290

8.3 Overview of the proposed genetic algorithm . . . . . . . . . . . . . . . . . . . . . . . . . . . . . .

8.4 Overview of proposed scheme . . . . . . . . . . . . . . . . . . . 299

8.4.1 Joint source and channel coding . . . . . . . . . . . . . . 301

8.4 .2 Iterative decoding . . . . . . . . . . . . . . . . . . . . . . . . . 302

8.5 Parameter design for the proposed scheme . . . . . . . . . . . . . 303

8.5.1 Design of IrVLC component VLEC codebook suites . . . . . . . . . 303

8.5.2 Characterisation of component VLEC codebooks . . . . . . . . . . . 307

8.5.3 Suitability of IrVLC component codebook suites . . . . . . . . . . . 311 
8.5.4 Parameterisations of the proposed scheme . . . . . . . . . . . 315

8.5 .5 Interleaver length . . . . . . . . . . . . . . . . . . . . . . . . . . . . . . . . . . . . . .

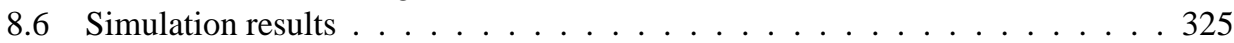

8.7 Summary and Conclusions . . . . . . . . . . . . . . . . . . 329

Chapter 9 Joint EXIT Chart Matching of Irregular Variable Length Coding and Irreg-

ular Unity Rate Coding 333

9.1 Introduction . . . . . . . . . . . . . . . . . . 333

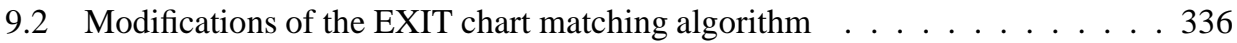

9.3 Joint EXIT chart matching . . . . . . . . . . . . . . . . . . . . . . . . . . . . . . . . . . . . .

9.4 Overview of the transmission scheme considered . . . . . . . . . . . 339

9.4.1 Joint source and channel coding . . . . . . . . . . . . . . . 339

9.4 .2 Iterative decoding . . . . . . . . . . . . . . . . . . . . . . . . . . . . . . . . . . 341

9.5 System parameter design . . . . . . . . . . . . . . . . . . . . . . . . . . . . . . . . . . . . . . . .

9.5.1 Component VLEC codebooks . . . . . . . . . . . . . . . . 343

9.5.2 Component URC codes . . . . . . . . . . . . . . . . . 346

9.5.3 EXIT chart matching . . . . . . . . . . . . . . . 350

9.5.4 Parameterisations of the proposed scheme . . . . . . . . . . . . 352

9.6 Simulation results . . . . . . . . . . . . . . . . . . . . . . . . . . . . . . . . . . . . . . . . . . . .

9.7 Summary and Conclusions . . . . . . . . . . . . . . . . 364

Chapter 10 Conclusions and Future Research 367

10.1 Chapter 1: Introduction . . . . . . . . . . . . . . . . . . . . . . . . . . . . . . . . . . . . . . .

10.2 Chapter 1: Information Theory Basics . . . . . . . . . . . . . . . . . . . . . . . . . . . . . . . . . . .

10.3 Chapter ??: Sources and Source Codes . . . . . . . . . . . . . . . . . . . . . . . . . . 368

10.4 Chapter ??: Iterative Source/Channel Decoding . . . . . . . . . . . . . . 370

10.5 Chapter ??: Three-Stage Serially Concatenated Turbo Equalisation . . . . . . 372

10.6 Chapter 6: Joint source and channel coding . . . . . . . . . . . . . . 375

10.7 Chapters 7 - 9: EXIT chart matching . . . . . . . . . . . . . 376

10.8 Chapter 8: GA-aided Design of Irregular VLC Components . . . . . . . . . . 379

10.9 Chapter 9: Joint EXIT Chart Matching of IRVLCs and IRURCs . . . . . . . 382

10.10Chapter ??: Iteratively Decoded VLC Space-Time Coded Modulation . . . . 389

10.11Chapter ??: Iterative Detection of Three-Stage Concatenated IrVLC FFH-

MFSK . . . . . . . . . . . . . . . . . . . . 389

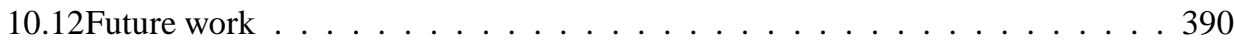

10.13 Closing remarks . . . . . . . . . . . . . . . . . 393

$\begin{array}{ll}\text { Bibliography } & 394\end{array}$

Author Index $\quad 409$

$\begin{array}{ll}\text { Glossary } & 409\end{array}$

$\begin{array}{ll}\text { Index } & 412\end{array}$ 


\section{List of Symbols}

\section{Schematics}

$\mathbf{f}_{n}$ Current video frame.

$\hat{\mathbf{f}}_{n}$ Quantised current video frame.

$\tilde{\mathbf{f}}_{n}$ Reconstructed current video frame.

$\hat{\mathbf{f}}_{n-1}$ Quantised previous video frame.

$\tilde{\mathbf{f}}_{n-1}$ Reconstructed previous video frame.

e Source sample frame.

ê Quantised source sample frame.

ẽ Reconstructed source sample frame.

s Source symbol frame.

s Reconstructed source symbol frame.

u Transmission frame.

$\hat{\mathbf{u}}$ Received transmission frame.

$\tilde{\mathbf{u}}$ Reconstructed transmission frame.

$\pi$ Interleaving.

$\pi^{-1}$ De-interleaving.

$\mathbf{u}^{\prime}$ Interleaved transmission frame.

$\mathbf{v}$ Encoded frame.

$\mathbf{v}^{\prime}$ Interleaved encoded frame.

$L_{a}(\cdot)$ A priori Logarithmic Likelihood Ratios (LLRs)/logarithmic A Posteriori Probabilities (Log-APPs) pertaining to the specified bits/symbols.

$L_{p}(\cdot)$ A posteriori LLRs/Log-APPs pertaining to the specified bits/symbols.

$L_{e}(\cdot)$ Extrinsic LLRs pertaining to the specified bits/symbols.

$\mathrm{x}$ Channel's input symbols.

y Channel's output symbols.

\section{Channel}

$\eta$ Effective throughput.

$E_{c} / N_{0}$ Channel Signal to Noise Ratio (SNR).

$E_{b} / N_{0}$ Channel SNR per bit of source information.

\section{Video blocks (VBs)}

$J_{x}^{\mathrm{MB}}$ Number of VB columns in each Macro-Block (MB).

$J_{y}^{\mathrm{MB}}$ Number of VB rows in each MB.

$J^{\mathrm{MB}}$ Number of VBs in each MB. 


\section{Sub-frames}

$M$ Number of sub-frames.

$m$ Sub-frame index.

$\mathbf{e}^{m}$ Source sample sub-frame.

$\hat{\mathbf{e}}^{m}$ Quantised source sample sub-frame.

$\tilde{\mathbf{e}}^{m}$ Reconstructed source sample sub-frame.

$\mathbf{u}^{m}$ Transmission sub-frame.

$\tilde{\mathbf{u}}^{m}$ Reconstructed transmission sub-frame.

$\mathbf{s}^{m}$ Source symbol sub-frame.

$\tilde{\mathbf{s}}^{m}$ Reconstructed source symbol sub-frame.

\section{Source sample sub-frames}

$J$ Number of source samples that are comprised by each source sample sub-frame.

$J^{\text {sum }}$ Number of source samples that are comprised by each source sample frame.

$j$ Source sample index.

$e_{j}^{m}$ Source sample.

$\hat{e}_{j}^{m}$ Quantised source sample.

$\tilde{e}_{j}^{m}$ Reconstructed source sample.

\section{Transmission sub-frames}

$I$ Number of bits that are comprised by each transmission sub-frame.

$I^{\text {sum }}$ Number of bits that are comprised by each transmission frame.

$I_{\min }$ Minimum number of bits that may be comprised by each transmission sub-frame.

$I_{\max }$ Maximum number of bits that may be comprised by each transmission sub-frame.

$i$ Transmission sub-frame bit index.

$u_{i}^{m}$ Transmission sub-frame bit.

$b$ Binary value.

\section{Codebooks}

$K$ Number of entries in the codebook.

$k$ Codebook entry index.

\section{VQ codebook}

VQ Vector Quantisation (VQ) codebook.

$\mathbf{V Q}^{k}$ VQ tile.

$J^{k}$ Number of VBs that are comprised by the VQ tile $\mathbf{V Q}^{k}$.

$j^{k}$ VQ tile VB index.

$V Q_{j^{k}}^{k}$ VQ tile VB. 


\section{VLC codebook}

VLC Variable Length Coding (VLC) codebook.

VLC $^{k}$ VLC codeword.

$I^{k}$ Number of bits that are comprised by the VLC codeword $\mathbf{V L C}^{k}$.

$I_{b}^{k}$ Number of bits in the VLC codeword $\mathbf{V L C}^{k}$ assuming a value $b \in\{0,1\}$.

$i^{k}$ VLC codeword bit index.

$V L C_{i^{k}}^{k}$ VLC codeword bit.

\section{VLC codebook parameters}

$E$ Entropy.

$L($ VLC) VLC codebook average codeword length.

$R$ (VLC) VLC coding rate.

$E(\mathbf{V L C})$ VLC-encoded bit entropy.

$T$ (VLC) VLC trellis complexity.

$O^{\mathrm{APP}}(\mathbf{V L C})$ Average number of Add, Compare and Select (ACS) operations performed per source symbol during A Posteriori Probability (APP) Soft-In Soft-Out (SISO) VLC decoding.

$O^{\mathrm{MAP}}(\mathbf{V L C})$ Average number of Add, Compare and Select (ACS) operations performed per source symbol during Maximum A posteriori Probability (MAP) VLC sequence estimation.

$d_{\text {free }}($ VLC) VLC codebook free distance.

$d_{b_{\min }}($ VLC) VLC codebook minimum block distance.

$d_{d_{\min }}(\mathbf{V L C})$ VLC codebook minimum divergence distance.

$d_{c_{\min }}(\mathbf{V L C})$ VLC codebook minimum convergence distance.

$\bar{d}_{\text {free }}($ VLC) VLC codebook free distance lower bound.

$D$ (VLC) VLC codebook Real-Valued Free Distance Metric (RV-FDM)

\section{Irregular Variable Length Coding (IrVLC)}

$N$ Component VLC codebook count.

$n$ Component VLC codebook index.

$\mathbf{u}^{n}$ Transmission sub-frame.

$\mathbf{s}^{n}$ Source symbol sub-frame.

$C^{n}$ Component VLC codebook source symbol frame fraction.

$\alpha^{n}$ Component VLC codebook transmission frame fraction.

\section{IrVLC codebooks}

VLC $^{n}$ Component VLC codebook.

VLC $^{n, k}$ Component VLC codeword.

$I^{n, k}$ Number of bits that are comprised by the component VLC codeword VLC ${ }^{n, k}$.

$i^{n, k}$ Component VLC codeword bit index.

$V L C_{i^{n, k}}^{k}$ Component VLC codeword bit. 
Irregular Unity Rate Coding (IrURC)

$R$ Component Unity Rate Code (URC) count.

$r$ Component URC index.

$\mathbf{u}^{\prime r}$ Interleaved transmission sub-frame.

$\mathbf{v}^{r}$ Encoded sub-frame.

URC $^{r}$ Component URC.

\section{Code parameters}

$R_{(\cdot)}$ Coding rate.

$M_{(\cdot)}$ Number of modulation constellation points.

$L_{(\cdot)}$ Coding memory.

\section{EXtrinsic Information Transfer (EXIT) chart}

$I_{a}$ A priori mutual information.

$I_{e}$ Extrinsic mutual information.

$y$ Importance of seeking a reduced computational complexity during EXIT chart matching.

\section{Trellises}

$\ddot{i}$ Bit state index.

$\ddot{j}$ Symbol state index.

$\ddot{n}$ Node state index.

$S_{(i, j)}$ Symbol-based trellis state.

$S_{(i, \ddot{n})}$ Bit-based trellis state.

\section{Trellis transitions}

$T$ Trellis transition.

$k^{T}$ Codebook entry index associated with the symbol-based trellis transition $T$.

$b^{T}$ Bit value represented by the bit-based trellis transition $T$.

$i^{T}$ Index of bit considered by the bit-based trellis transition $T$.

$\ddot{i}^{T}$ Bit state index of the trellis state that the symbol-based trellis transition $T$ emerges from.

$\ddot{j}^{T}$ Symbol state index of the trellis state that the symbol-based trellis transition $T$ emerges from.

$\ddot{n}^{T}$ Node state index of the trellis state that the bit-based trellis transition $T$ emerges from.

\section{Trellis sets}

en $\left(u_{i}^{m}\right)$ The set of all trellis transitions that encompasses the transmission sub-frame bit $u_{i}^{m}$. en $\left(u_{i}^{m}=b\right)$ The sub-set of en $\left(u_{i}^{m}\right)$ that maps the binary value $b$ to the transmission subframe bit $u_{i}^{m}$.

en $\left(\hat{e}_{j}^{m}\right)$ The set of all trellis transitions that encompasses the $\operatorname{VB} \hat{e}_{j}^{m}$.

en $\left(\hat{e}_{j}^{m}=V Q_{j^{k}}^{k}\right)$ The sub-set of en $\left(\hat{e}_{j}^{m}\right)$ that maps the VQ tile $V Q_{j^{k}}^{k}$ to the VB $\hat{e}_{j}^{m}$. 
$\operatorname{fr}(S)$ The set of all transitions that emerge from the trellis state $S$.

to $(S)$ The set of all transitions that merge to the trellis state $S$.

$\operatorname{fr}(T)$ The state that the transition $T$ emerges from.

to $(T)$ The state that the transition $T$ merges to.

$\operatorname{nr}\left(\hat{e}_{j}^{m}\right)$ The set of all VBs that immediately surround the $\operatorname{VB} \hat{e}_{j}^{m}$.

\section{Viterbi algorithm}

$d(T)$ The distortion of the trellis transition $T$.

$D(T)$ The minimum cumulative distortion of all trellis paths between the trellis state $S_{(0,0)}$ and the trellis transition $T$.

$D(S)$ The minimum cumulative distortion of all trellis paths to the state $S$.

$m(T)$ The Viterbi algorithm metric of the trellis transition $T$.

$M(T)$ The maximum cumulative Viterbi algorithm metric of all trellis paths between the trellis state $S_{(0,0)}$ and the trellis transition $T$.

$M(S)$ The maximum cumulative Viterbi algorithm metric of all trellis paths to the state $S$.

$M(S)$ The maximum cumulative Viterbi algorithm metric of all trellis paths to the sate $S$.

\section{Bahl-Cocke-Jelinek-Raviv (BCJR) algorithm}

$P_{a}\left(u_{i}^{m}=b\right)$ A priori probability of the transmission sub-frame bit $u_{i}^{m}$ taking the value $b$.

$P(k)$ Probability of occurrence of the codebook entry with index $k$.

$P(S)$ Probability of occurrence of the trellis state $S$.

$P(T \mid \operatorname{fr}(T))$ Conditional probability of the occurrence of the trellis transition $T$ given the occurrence of the trellis state that it emerges from.

$P_{p}(T)$ A posteriori trellis transition probability.

$C_{1}$ A posteriori trellis transition probability normalisation factor.

$\gamma(T)$ A priori trellis transition probability.

$\gamma^{\prime}(T)$ Weighted a priori trellis transition probability.

$C_{2}(S)$ A priori trellis transition probability normalisation factor used for all trellis transitions that emerge from the trellis state $S$.

$\alpha(S)$ Alpha value obtained for the trellis state $S$.

$\beta(S)$ Beta value obtained for the trellis state $S$.

$C_{L_{a}}$ BCJR algorithm LLR pruning threshold.

$C_{\gamma}$ BCJR algorithm a priori probability pruning threshold.

$C_{\alpha}$ BCJR algorithm forward recursion pruning threshold.

$C_{\beta}$ BCJR algorithm backwards recursion pruning threshold.

\section{Genetic Algorithm (GA) for VLC codebook design}

L List of candidate VLC codebooks.

$L^{\text {tar }}$ Target GA list length.

$M$ (VLC) GA VLC quality metric.

$D^{\lim }$ GA VLC RV-FDM limit.

$R^{\lim }$ GA VLC coding rate limit. 
$\alpha^{D}$ GA VLC RV-FDM importance.

$\alpha^{R}$ GA VLC coding rate importance.

$\alpha^{E}$ GA VLC bit entropy importance.

$\alpha^{T}$ GA VLC trellis complexity importance.

$\beta^{D}$ GA VLC RV-FDM increase/decrease constant.

$\beta^{R}$ GA VLC coding rate increase/decrease constant.

$D^{\text {best }}$ Most desirable RV-FDM of VLC codebooks admitted to the GA list.

$R^{\text {best }}$ Most desirable coding rate of VLC codebooks admitted to the GA list.

$E^{\text {best }}$ Most desirable bit entropy of VLC codebooks admitted to the GA list.

$T^{\text {best }}$ Most desirable trellis complexity of VLC codebooks admitted to the GA list.

$P^{\max }$ Maximum number of GA mutations. 


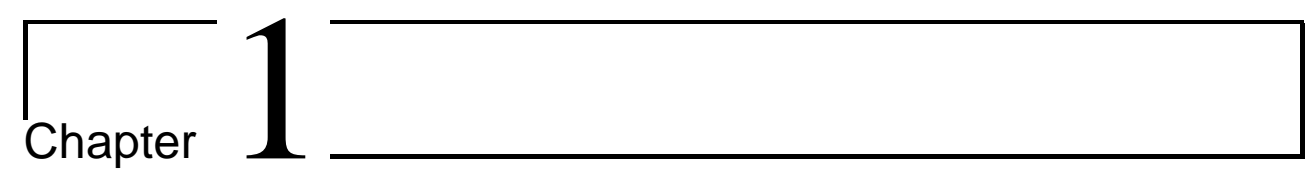

\section{Information Theory Basics}

\subsection{Issues in Information Theory}

The ultimate aim of telecommunications is to communicate information between two geographically separated locations via a communications channel with adequate quality. The theoretical foundations of information theory accrue from Shannon's pioneering work [24-27], and hence most tutorial interpretations of his work over the past fifty years rely fundamentally on [24-27]. This chapter is no exception in this respect. Throughout this chapter we make frequent references to Shannon's seminal papers and to the work of various authors offering further insights into Shannonian information theory. Since this monograph aims to provide an all-encompassing coverage of video compression and communications, we begin by addressing the underlying theoretical principles using a light-hearted approach, often relying on worked examples.

Early forms of human telecommunications were based on smoke, drum or light signals, bonfires, semaphores, and the like. Practical information sources can be classified as analog and digital. The output of an analog source is a continuous function of time, such as, for example, the air pressure variation at the membrane of a microphone due to someone talking. The roots of Nyquist's sampling theorem are based on his observation of the maximum achievable telegraph transmission rate over bandlimited channels [28]. In order to be able to satisfy Nyquist's sampling theorem the analogue source signal has to be bandlimited before sampling. The analog source signal has to be transformed into a digital representation with the aid of time- and amplitude-discretization using sampling and quantization.

The output of a digital source is one of a finite set of ordered, discrete symbols often referred to as an alphabet. Digital sources are usually described by a range of characteristics, such as the source alphabet, the symbol rate, the symbol probabilities, and the probabilistic interdependence of symbols. For example, the probability of $u$ following $q$ in the English language is $p=1$, as in the word "equation." Similarly, the joint probability of all pairs of consecutive symbols can be evaluated.

In recent years, electronic telecommunications have become prevalent, although most information sources provide information in other forms. For electronic telecommunications, 
the source information must be converted to electronic signals by a transducer. For example, a microphone converts the air pressure waveform $p(t)$ into voltage variation $v(t)$, where

$$
v(t)=c \cdot p(t-\tau),
$$

and the constant $c$ represents a scaling factor, while $\tau$ is a delay parameter. Similarly, a video camera scans the natural three-dimensional scene using optics and converts it into electronic waveforms for transmission.

The electronic signal is then transmitted over the communications channel and converted back to the required form, which may be carried out, for example, by a loudspeaker. It is important to ensure that the channel conveys the transmitted signal with adequate quality to the receiver in order to enable information recovery. Communications channels can be classified according to their ability to support analog or digital transmission of the source signals in a simplex, duplex, or half-duplex fashion over fixed or mobile physical channels constituted by pairs of wires, Time Division Multiple Access (TDMA) time-slots, or a Frequency Division Multiple Access (FDMA) frequency slot.

The channel impairments may include superimposed, unwanted random signals, such as thermal noise, crosstalk via multiplex systems from other users, man-made interference from car ignition, fluorescent lighting, and other natural sources such as lightning. Just as the natural sound pressure wave between two conversing persons will be impaired by the acoustic background noise at a busy railway station, similarly the reception quality of electronic signals will be affected by the above unwanted electronic signals. In contrast, distortion manifests itself differently from additive noise sources, since no impairment is explicitly added. Distortion is more akin to the phenomenon of reverberating loudspeaker announcements in a large, vacant hall, where no noise sources are present.

Some of the channel impairments can be mitigated or counteracted; others cannot. For example, the effects of unpredictable additive random noise cannot be removed or "subtracted" at the receiver. Its effects can be mitigated by increasing the transmitted signal's power, but the transmitted power cannot be increased without penalties, since the system's nonlinear distortion rapidly becomes dominant at higher signal levels. This process is similar to the phenomenon of increasing the music volume in a car parked near a busy road to a level where the amplifier's distortion becomes annoyingly dominant.

In practical systems, the Signal-to-Noise Ratio (SNR) quantifying the wanted and unwanted signal powers at the channel's output is a prime channel parameter. Other important channel parameters are its amplitude and phase response, determining its usable bandwidth $(B)$, over which the signal can be transmitted without excessive distortion. Among the most frequently used statistical noise properties are the probability density function (PDF), cumulative density function (CDF), and power spectral density (PSD).

The fundamental communications system design considerations are whether a high-fidelity (HI-FI) or just acceptable video or speech quality is required from a system, which predetermines, among other factors, its cost, bandwidth requirements, as well as the number of channels available, and has implementational complexity ramifications. Equally important are the issues of robustness against channel impairments, system delay, and so on. The required transmission range and worldwide roaming capabilities, the maximum available transmission speed in terms of symbols/sec, information confidentiality, reception reliability, convenient 


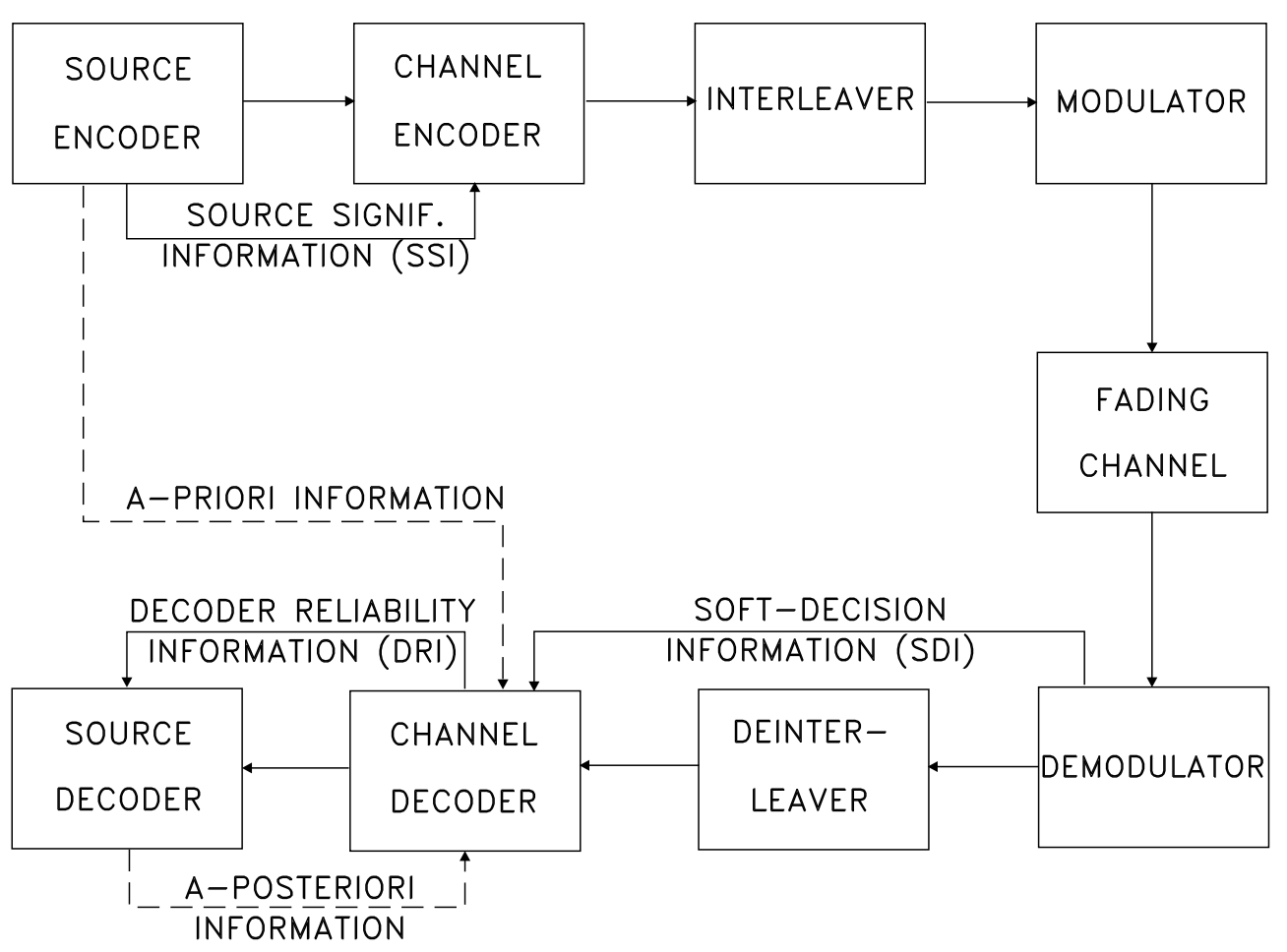

Figure 1.1: Basic transmission model of information theory.

lightweight, solar-charged design, are similarly salient characteristics of a communications system.

Information theory deals with a variety of problems associated with the performance limits of the information transmission system, such as that depicted in Figure 1.1. The components of this system constitute the subject of this monograph; hence they will be treated in greater depth later in this volume. Suffice it to say at this stage that the transmitter seen in Figure 1.1 incorporates a source encoder, a channel encoder, an interleaver, and a modulator and their inverse functions at the receiver. The ideal source encoder endeavors to remove as much redundancy as possible from the information source signal without affecting its source representation fidelity (i.e., distortionlessly), and it remains oblivious of such practical constraints as a finite delay and limited signal processing complexity. In contrast, a practical source encoder will have to retain a limited signal processing complexity and delay while attempting to reduce the source representation bit rate to as low a value as possible. This operation seeks to achieve better transmission efficiency, which can be expressed in terms of bit-rate economy or bandwidth conservation.

The channel encoder re-inserts redundancy or parity information but in a controlled manner in order to allow error correction at the receiver. Since this component is designed to ensure the best exploitation of the re-inserted redundancy, it is expected to minimize the error 
probability over the most common channel, namely, the so-called Additive White Gaussian Noise (AWGN) channel, which is characterized by a memoryless, random distribution of channel errors. However, over wireless channels, which have recently become prevalent, the errors tend to occur in bursts due to the presence of deep received signal fades induced by the distructively superimposed multipath phenomena. This is why our schematic of Figure 1.1 contains an interleaver block, which is included in order to randomize the bursty channel errors. Finally, the modulator is designed to ensure the most bandwidth-efficient transmission of the source- and channel encoded, interleaved information stream, while maintaining the lowest possible bit error probability. The receiver simply carries out the corresponding inverse functions of the transmitter. Observe in the figure that besides the direct interconnection of the adjacent system components there are a number of additional links in the schematic, which will require further study before their role can be highlighted. Thus, at the end of this chapter we will return to this figure and guide the reader through its further intricate details.

Some fundamental problems transpiring from the schematic of Figure 1.1, which were addressed in depth by a range of references due to Shannon [24-27], Nyquist [28], Hartley [29], Abramson [30], Carlson [31], Raemer [32], and Ferenczy [33] and others are as follows:

- What is the true information generation rate of our information sources? If we know the answer, the efficiency of coding and transmission schemes can be evaluated by comparing the actual transmission rate used with the source's information emission rate. The actual transmission rate used in practice is typically much higher than the average information delivered by the source, and the closer these rates are, the better is the coding efficiency.

- Given a noisy communications channel, what is the maximum reliable information transmission rate? The thermal noise induced by the random motion of electrons is present in all electronic devices, and if its power is high, it can seriously affect the quality of signal transmission, allowing information transmission only at low-rates.

- Is the information emission rate the only important characteristic of a source, or are other message features, such as the probability of occurrence of a message and the joint probability of occurrence for various messages, also important?

- In a wider context, the topic of this whole monograph is related to the blocks of Figure 1.1 and to their interactions, but in this chapter we lay the theoretical foundations of source and channel coding as well as transmission issues and define the characteristics of an ideal Shannonian communications scheme.

Although numerous excellent treatises are available on these topics, which treat the same subjects with a different flavor [31,33,34], our approach is similar to that of the above classic sources; since the roots are in Shannon's work, references [24-27,35,36] are the most pertinent and authoritative sources.

In this chapter we consider mainly discrete sources, in which each source message is associated with a certain probability of occurrence, which might or might not be dependent on previous source messages. Let us now give a rudimentary introduction to the characteristics of the AWGN channel, which is the predominant channel model in information theory due to its simplicity. The analytically less tractable wireless channels will be modeled mainly by simulations in this monograph 


\subsection{Additive White Gaussian Noise Channel}

\subsubsection{Background}

In this section, we consider the communications channel, which exists between the transmitter and the receiver, as shown in Figure 1.1. Accurate characterization of this channel is essential if we are to remove the impairments imposed by the channel using signal processing at the receiver. Here we initially consider only fixed communications links whereby both terminals are stationary, although mobile radio communications channels, which change significantly with time, are becoming more prevalent.

We define fixed communications channels to be those between a fixed transmitter and a fixed receiver. These channels are exemplified by twisted pairs, cables, wave guides, optical fiber and point-to-point microwave radio channels. Whatever the nature of the channel, its output signal differs from the input signal. The difference might be deterministic or random, but it is typically unknown to the receiver. Examples of channel impairments are dispersion, nonlinear distortions, delay, and random noise.

Fixed communications channels can often be modeled by a linear transfer function, which describes the channel dispersion. The ubiquitous additive Gaussian noise (AWGN) is a fundamental limiting factor in communications via linear time-invariant (LTI) channels. Although the channel characteristics might change due to factors such as aging, temperature changes, and channel switching, these variations will not be apparent over the course of a typical communication session. It is this inherent time invariance that characterizes fixed channels.

An ideal, distortion-free communications channel would have a flat frequency response and linear phase response over the frequency range of $-\infty \ldots+\infty$, although in practice it is sufficient to satisfy this condition over the bandwidth $(B)$ of the signals to be transmitted, as seen in Figure 1.2. In this figure, $A(\omega)$ represents the magnitude of the channel response at frequency $w$, and $\phi(w)=w T$ represents the phase shift at frequency $w$ due to the circuit delay $T$.

Practical channels always have some linear distortions due to their bandlimited, nonflat frequency response and nonlinear phase response. In addition, the group-delay response of the channel, which is the derivative of the phase response, is often given.

\subsubsection{Practical Gaussian Channels}

Conventional telephony uses twisted copper wire pairs to connect subscribers to the local exchange. The bandwidth is approximately $3.4 \mathrm{kHz}$, and the waveform distortions are relatively benign.

For applications requiring a higher bandwidth, coaxial cables can be used. Their attenuation increases approximately with the square root of the frequency. Hence, for wideband, long-distance operation, they require channel equalization. Typically, coaxial cables can provide a bandwidth of about $50 \mathrm{MHz}$, and the transmission rate they can support is limited by the so-called skin effect.

Point-to-point microwave radio channels typically utilize high-gain directional transmit and receive antennas in a line-of-sight scenario, where free-space propagation conditions may be applicable. 


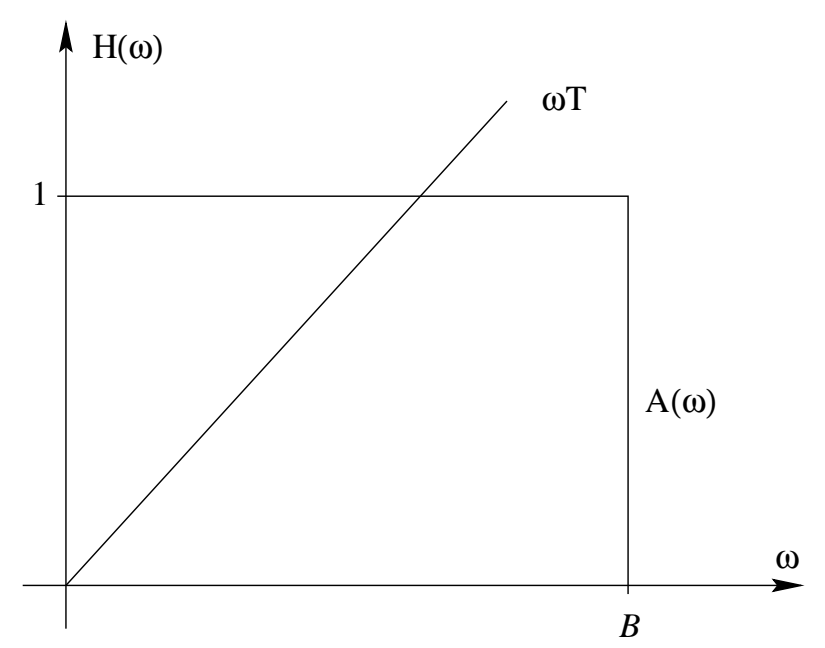

Figure 1.2: Ideal, distortion-free channel model having a linear phase and a flat magnitude response.

\subsubsection{Gaussian Noise}

Regardless of the communications channel used, random noise is always present. Noise can be broadly classified as natural or man-made. Examples of man-made noise are those due to electrical appliances, and fluorescent lighting, and the effects of these sources can usually be mitigated at the source. Natural noise sources affecting radio transmissions include galactic star radiations and atmospheric noise. There exists a low-noise frequency window in the range of $1-10 \mathrm{GHz}$, where the effects of these sources are minimized.

Natural thermal noise is ubiquitous. This is due to the random motion of electrons, and it can be reduced by reducing the temperature. Since thermal noise contains practically all frequency components up to some $10^{13} \mathrm{~Hz}$ with equal power, it is often referred to as white noise (WN) in an analogy to white light containing all colors with equal intensity. This WN process can be characterized by its uniform power spectral density (PSD) $N(\omega)=N_{0} / 2$ shown together with its autocorrelation function (ACF) in Figure 1.3.

The power spectral density of any signal can be conveniently measured by the help of a selective narrowband power meter tuned across the bandwidth of the signal. The power measured at any frequency is then plotted against the measurement frequency. The autocorrelation function $R(\tau)$ of the signal $x(t)$ gives an average indication of how predictable the signal $x(t)$ is after a period of $\tau$ seconds from its present value. Accordingly, it is defined as follows:

$$
R(\tau)=\lim _{T \rightarrow \infty} \frac{1}{T} \int_{-\infty}^{\infty} x(t) x(t+\tau) d t
$$

For a periodic signal $x(t)$, it is sufficient to evaluate the above equation for a single period $T_{0}$, yielding:

$$
R(\tau)=\frac{1}{T_{0}} \int_{-T_{0} / 2}^{T_{0} / 2} x(t) x(t+\tau) d t
$$




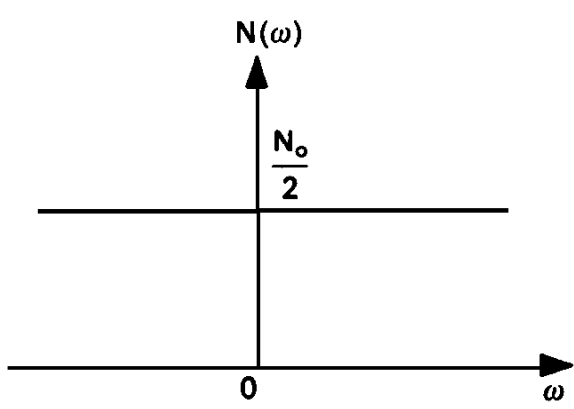

(a)

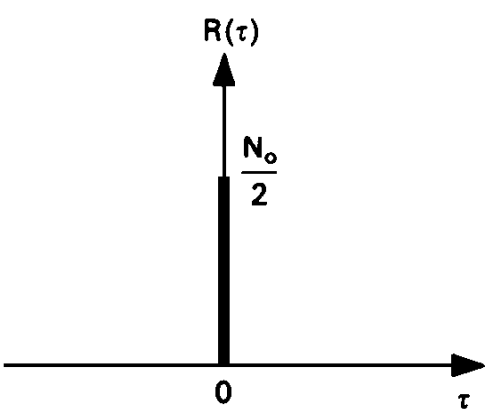

(b)

Figure 1.3: Power spectral density and autocorrelation of WN.

The basic properties of the ACF are:

- The ACF is symmetric: $R(\tau)=R(-\tau)$.

- The ACF is monotonously decreasing: $R(\tau) \leq R(0)$.

- For $\tau=0$ we have $R(0)=\overline{x^{2}}(t)$, which is the signal's power.

- The ACF and the PSD form a Fourier transform pair, which is formally stated as the Wiener-Khintchine theorem, as follows:

$$
\begin{aligned}
R(\tau) & =\frac{1}{2 \pi} \int_{-\infty}^{\infty} N(\omega) e^{j \omega \tau} d \omega \\
& =\frac{1}{2 \pi} \int_{-\infty}^{\infty} \frac{N_{0} e^{j \omega \tau}}{2} d \omega \\
& =\frac{1}{2 \pi} \frac{N_{0}}{2} \int_{-\infty}^{\infty} e^{j \omega \tau} d \omega=\frac{N_{0}}{2} \delta(\tau),
\end{aligned}
$$

where $\delta(\tau)$ is the Dirac delta function. Clearly, for any timed-domain shift $\tau>0$, the noise is uncorrelated.

Bandlimited communications systems bandlimit not only the signal but the noise as well, and this filtering limits the rate of change of the time-domain noise signal, introducing some correlation over the interval of $\pm 1 / 2 B$. The stylized PSD and ACF of bandlimited white noise are displayed in Figure 1.4. 

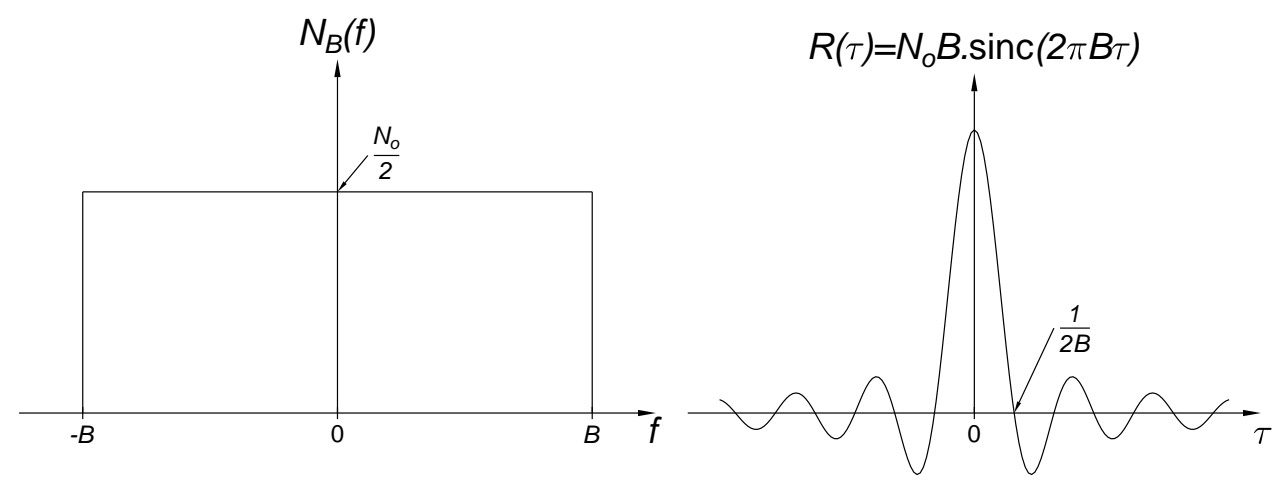

Figure 1.4: Power spectral density and autocorrelation of bandlimited WN.

After bandlimiting, the autocorrelation function becomes:

$$
\begin{aligned}
R(\tau) & =\frac{1}{2 \pi} \int_{-B}^{B} \frac{N_{0}}{2} e^{j \omega \tau} d \omega=\frac{N_{0}}{2} \int_{-B}^{B} e^{j 2 \pi f \tau} d f \\
& =\frac{N_{0}}{2}\left[\frac{e^{j 2 \pi f \tau}}{j 2 \pi \tau}\right]_{-B}^{B} \\
& =\frac{1}{j 2 \pi \tau}[\cos 2 \pi B \tau+j \sin 2 \pi B \tau-\cos 2 \pi B \tau+j \sin 2 \pi B \tau] \\
& =N_{0} B \frac{\sin (2 \pi B \tau)}{2 \pi B \tau}
\end{aligned}
$$

which is the well-known sinc-function seen in Figure 1.4.

In the time-domain, the amplitude distribution of the white thermal noise has a normal or Gaussian distribution, and since it is inevitably added to the received signal, it is usually referred to as additive white Gaussian noise (AWGN). Note that AWGN is therefore the noise generated in the receiver. The probability density function (PDF) is the well-known bell-shaped curve of the Gaussian distribution, given by

$$
p(x)=\frac{1}{\sigma \sqrt{2 \pi}} e^{-(x-m) / 2 \sigma^{2}}
$$

where $m$ is the mean and $\sigma^{2}$ is the variance. The effects of AWGN can be mitigated by increasing the transmitted signal power and thereby reducing the relative effects of noise. The signal-to-noise ratio (SNR) at the receiver's input provides a good measure of the received signal quality. This SNR is often referred to as the channel SNR.

\subsection{Information of a Source}

Based on Shannon's work [24-27,35,36], let us introduce the basic terms and definitions of information theory by considering a few simple examples. Assume that a simple 8-bit 
analog-to-digital (ADC) converter emits a sequence of mutually independent source symbols that can take the values $i=1,2, \ldots 256$ with equal probability. One may wonder, how much information can be inferred upon receiving one of these samples? It is intuitively clear that this inferred information is definitely proportional to the "uncertainty" resolved by the reception of one such symbol, which in turn implies that the information conveyed is related to the number of levels in the ADC. More explicitly, the higher the number of legitimate quantization levels, the lower the relative frequency or probability of receiving any one of them and hence the more "surprising," when any one of them is received. Therefore, less probable quantized samples carry more information than their more frequent, more likely counterparts.

Not suprisingly, one could resolve this uncertainty by simply asking a maximum of 256 questions, such as "Is the level 1?" "Is it 2? ..." "Is it 256?" Following Hartley's approach [29], a more efficient strategy would be to ask eight questions, such as: "Is the level larger than 128?" No. "Is it larger than 64?" No. ... "Is it larger than 2?" No. "Is it larger than 1?" No. Clearly, the source symbol emitted was of magnitude one, provided that the zero level was not used. We could therefore infer that $\log _{2} 256=8$ "Yes/No" binary answers were needed to resolve any uncertainty as regards the source symbol's level.

In more general terms, the information carried by any one symbol of a $q$-level source, where all the levels are equiprobable with probabilities of $p_{i}=1 / q, i=1 \ldots q$, is defined as

$$
I=\log _{2} q
$$

Rewriting Equation 1.7 using the message probabilities $p_{i}=\frac{1}{q}$ yields a more convenient form:

$$
I=\log _{2} \frac{1}{p_{i}}=-\log _{2} p_{i}
$$

which now is also applicable in case of arbitrary, unequal message probabilities $p_{i}$, again, implying the plausible fact that the lower the probability of a certain source symbol, the higher the information conveyed by its occurrence. Observe, however, that for unquantized analog sources, where as regards to the number of possible source symbols we have $q \rightarrow \infty$ and hence the probability of any analog sample becomes infinitesimally low, these definitions become meaningless.

Let us now consider a sequence of $N$ consecutive $q$-ary symbols. This sequence can take $q^{N}$ number of different values, delivering $q^{N}$ different messages. Therefore, the information carried by one such sequence is:

$$
I_{N}=\log _{2}\left(q^{N}\right)=N \log _{2} q,
$$

which is in perfect harmony with our expectation, delivering $N$ times the information of a single symbol, which was quantified by Equation 1.7. Doubling the sequence length to $2 \mathrm{~N}$ carries twice the information, as suggested by:

$$
I_{2 N}=\log _{2}\left(q^{2 N}\right)=2 N \cdot \log _{2} q .
$$

Before we proceed, let us briefly summarize the basic properties of information following Shannon's work [24-27, 35, 36]: 
- If for the probability of occurrences of the symbols $j$ and $k$ we have $p_{j}<p_{k}$, then as regards the information carried by them we have: $I(k)<I(j)$.

- If in the limit we have $p_{k} \rightarrow 1$, then for the information carried by the symbol $k$ we have $I(k) \rightarrow 0$, implying that symbols, whose probability of occurrence tends to unity, carry no information.

- If the symbol probability is in the range of $0 \leq p_{k} \leq 1$, then as regards the information carried by it we have $I(k) \geq 0$.

- For independent messages $k$ and $j$, their joint information is given by the sum of their information: $I(k, j)=I(k)+I(j)$. For example, the information carried by the statement "My son is 14 years old and my daughter is 12 " is equivalent to that of the sum of these statements: "My son is 14 years old" and "My daughter is 12 years old."

- In harmony with our expectation, if we have two equiprobable messages 0 and 1 with probabilities, $p_{1}=p_{2}=\frac{1}{2}$, then from Equation 1.8 we have $I(0)=I(1)=1$ bit.

\subsection{Average Information of Discrete Memoryless Sources}

Following Shannon's approach [24-27, 35, 36], let us now consider a source emitting one of $q$ possible symbols from the alphabet $s=s_{1}, s_{2}, \ldots s_{i} \ldots s_{q}$ having symbol probabilities of $p_{i}, i=1,2, \ldots q$. Suppose that a long message of $N$ symbols constituted by symbols from the alphabet $s=s_{1}, s_{2}, \ldots s_{q}$ having symbol probabilities of $p_{i}$ is to be transmitted. Then the symbol $s_{i}$ appears in every $N$-symbol message on the average $p_{i} \cdot N$ number of times, provided the message length is sufficiently long. The information carried by symbol $s_{i}$ is $\log _{2} 1 / p_{i}$ and its $p_{i} \cdot N$ occurrences yield an information contribution of

$$
I(i)=p_{i} \cdot N \cdot \log _{2} \frac{1}{p_{i}}
$$

Upon summing the contributions of all the $q$ symbols, we acquire the total information carried by the $N$-symbol sequence:

$$
I=\sum_{i=1}^{q} p_{i} N \cdot \log _{2} \frac{1}{p_{i}}[\text { bits }] .
$$

Averaging this over the $N$ symbols of the sequence yields the average information per symbol, which is referred to as the source's entropy [25] :

$$
H=\frac{I}{N}=\sum_{i=1}^{q} p_{i} \cdot \log _{2} \frac{1}{p_{i}}=-\sum_{i=1}^{q} p_{i} \log _{2} p_{i}[\text { bit/symbol }]
$$

Then the average source information rate can be defined as the product of the information carried by a source symbol, given by the entropy $H$ and the source emission rate $R_{s}$ :

$$
\left.R=R_{s} \cdot H \text { [bits/sec }\right] .
$$


Observe that Equation 1.13 is analogous to the discrete form of the first moment or in other words the mean of a random process with a probability density function (PDF) of $p(x)$, as in

$$
\bar{x}=\int_{-\infty}^{\infty} x \cdot p(x) d x,
$$

where the averaging corresponds to the integration, and the instantaneous value of the random variable $x$ represents the information $\log _{2} p_{i}$ carried by message $i$, which is weighted by its probability of occurrence $p_{i}$ quantified for a continuous variable $x$ by $p(x)$.

\subsubsection{Maximum Entropy of a Binary Source}

Let us assume that a binary source, for which $q=2$, emits two symbols with probabilities $p_{1}=p$ and $p_{2}=(1-p)$, where the sum of the symbol probabilities must be unity. In order to quantify the maximum average information of a symbol from this source as a function of the symbol probabilities, we note from Equation 1.13 that the entropy is given by:

$$
H(p)=-p \cdot \log _{2} p-(1-p) \cdot \log _{2}(1-p) .
$$

As in any maximization problem, we set $\partial H(p) / \partial p=0$, and upon using the differentiation chain rule of $(u \cdot v)^{\prime}=u^{\prime} \cdot v+u \cdot v^{\prime}$ as well as exploiting that $\left(\log _{a} x\right)^{\prime}=\frac{1}{x} \log _{a} e$ we arrive at:

$$
\begin{aligned}
\frac{\partial H(p)}{\partial p} & =-\log _{2} p-\frac{p}{p} \cdot \log _{2} e+\log _{2}(1-p)+\frac{(1-p)}{(1-p)} \log _{2} e=0 \\
\log _{2} p & =\log _{2}(1-p) \\
p & =(1-p) \\
p & =0.5
\end{aligned}
$$

This result suggests that the entropy is maximum for equiprobable binary messages. Plotting Equation 1.16 for arbitrary $p$ values yields Figure 1.5, in which Shannon suggested that the average information carried by a symbol of a binary source is low, if one of the symbols has a high probability, while the other a low probability.

Example: Let us compute the entropy of the binary source having message probabilities of $p_{1}=\frac{1}{8}, p_{2}=\frac{7}{8}$.

The entropy is expressed as:

$$
H=-\frac{1}{8} \log _{2} \frac{1}{8}-\frac{7}{8} \log _{2} \frac{7}{8} .
$$

Exploiting the following equivalence:

$$
\log _{2}(x)=\log _{10}(x) \cdot \log _{2}(10) \approx 3.322 \cdot \log _{10}(x)
$$

we have:

$$
H \approx \frac{3}{8}-\frac{7}{8} \cdot 3.322 \cdot \log _{10} \frac{7}{8} \approx 0.54[\mathrm{bit} / \mathrm{symbol}]
$$




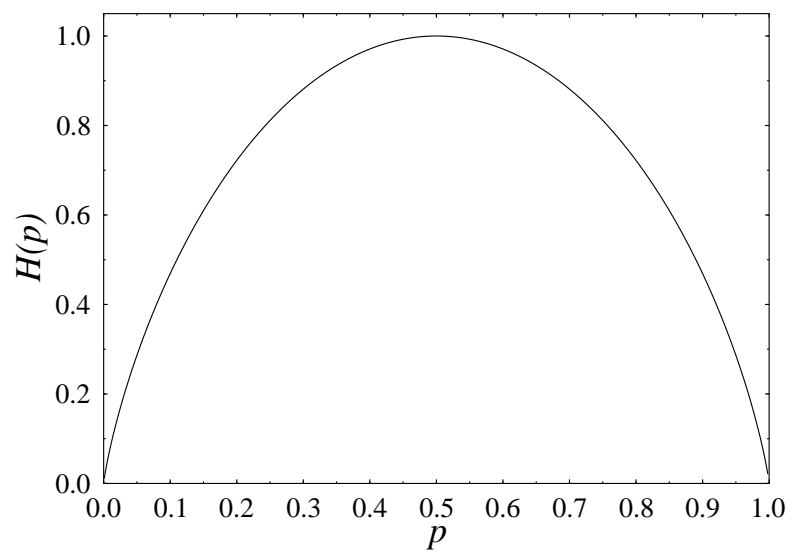

Figure 1.5: Entropy versus message probability $p$ for a binary source. (C)Shannon [25], BSTJ, 1948.

again implying that if the symbol probabilities are rather different, the entropy becomes significantly lower than the achievable $1 \mathrm{bit} / \mathrm{symbol}$. This is because the probability of encountering the more likely symbol is so close to unity that it carries hardly any information, which cannot be compensated by the more "informative" symbol's reception. For the even more unbalanced situation of $p_{1}=0.1$ and $p_{2}=0.9$ we have:

$$
\begin{aligned}
H & =-0.1 \log _{2} 0.1-0.9 \cdot \log _{2} 0.9 \\
& \approx-\left(0.3322 \cdot \log _{10} 0.1+0.9 \cdot 3.322 \cdot \log _{10} 0.9\right) \\
& \approx 0.3322+0.1368 \\
& \approx 0.47[\mathrm{bit} / \text { symbol] } .
\end{aligned}
$$

In the extreme case of $p_{1}=0$ or $p_{2}=1$ we have $H=0$. As stated before, the average source information rate is defined as the product of the information carried by a source symbol, given by the entropy $H$ and the source emission rate $R_{s}$, yielding $R=R_{s} \cdot H$ [bits/sec]. Transmitting the source symbols via a perfect noiseless channel yields the same received sequence without loss of information.

\subsubsection{Maximum Entropy of a $q$-ary Source}

For a $q$-ary source the entropy is given by:

$$
H=-\sum_{i=1}^{q} p_{i} \log _{2} p_{i},
$$

where, again, the constraint $\sum p_{i}=1$ must be satisfied. When determining the extreme value of the above expression for the entropy $H$ under the constraint of $\sum p_{i}=1$, the 
following term has to be maximized:

$$
D=\sum_{i=1}^{q}-p_{i} \log _{2} p_{i}+\lambda \cdot\left[1-\sum_{i=1}^{q} p_{i}\right],
$$

where $\lambda$ is the so-called Lagrange multiplier. Following the standard procedure of maximizing an expression, we set:

$$
\frac{\partial D}{\partial p_{i}}=-\log _{2} p_{i}-\frac{p_{i}}{p_{i}} \cdot \log _{2} e-\lambda=0
$$

leading to

$$
\log _{2} p_{i}=-\left(\log _{2} e+\lambda\right)=\text { Constant for } i=1 \ldots q,
$$

which implies that the maximum entropy of a $q$-ary source is maintained, if all message probabilities are identical, although at this stage the value of this constant probability is not explicit. Note, however, that the message probabilites must sum to unity, and hence:

$$
\sum_{i=1}^{q} p_{i}=1=q \cdot a,
$$

where $a$ is a constant, leading to $a=1 / q=p_{i}$, implying that the entropy of any $q$-ary source is maximum for equiprobable messages. Furthermore, $H$ is always bounded according to:

$$
0 \leq H \leq \log _{2} q
$$

\subsection{Source Coding for a Discrete Memoryless Source}

Interpreting Shannon's work [24-27,35,36] further, we see that source coding is the process by which the output of a $q$-ary information source is converted to a binary sequence for transmission via binary channels, as seen in Figure 1.1. When a discrete memoryless source generates $q$-ary equiprobable symbols with an average information rate of $R=R_{s} \log _{2} q$, all symbols convey the same amount of information, and efficient signaling takes the form of binary transmissions at a rate of $R$ bps. When the symbol probabilities are unequal, the minimum required source rate for distortionless transmission is reduced to

$$
R=R_{s} \cdot H<R_{s} \log _{2} q .
$$

Then the transmission of a highly probable symbol carries little information and hence assigning $\log _{2} q$ number of bits to it does not use the channel efficiently. What can be done to improve transmission efficiency? Shannon's source coding theorem suggests that by using a source encoder before transmission the efficiency of the system with equiprobable source symbols can be arbitrarily approached. 
Algorithm 1 (Shannon-Fano Coding) This algorithm summarizes the Shannon-Fano coding steps. (See also Figure 1.6 and Table 1.1.)

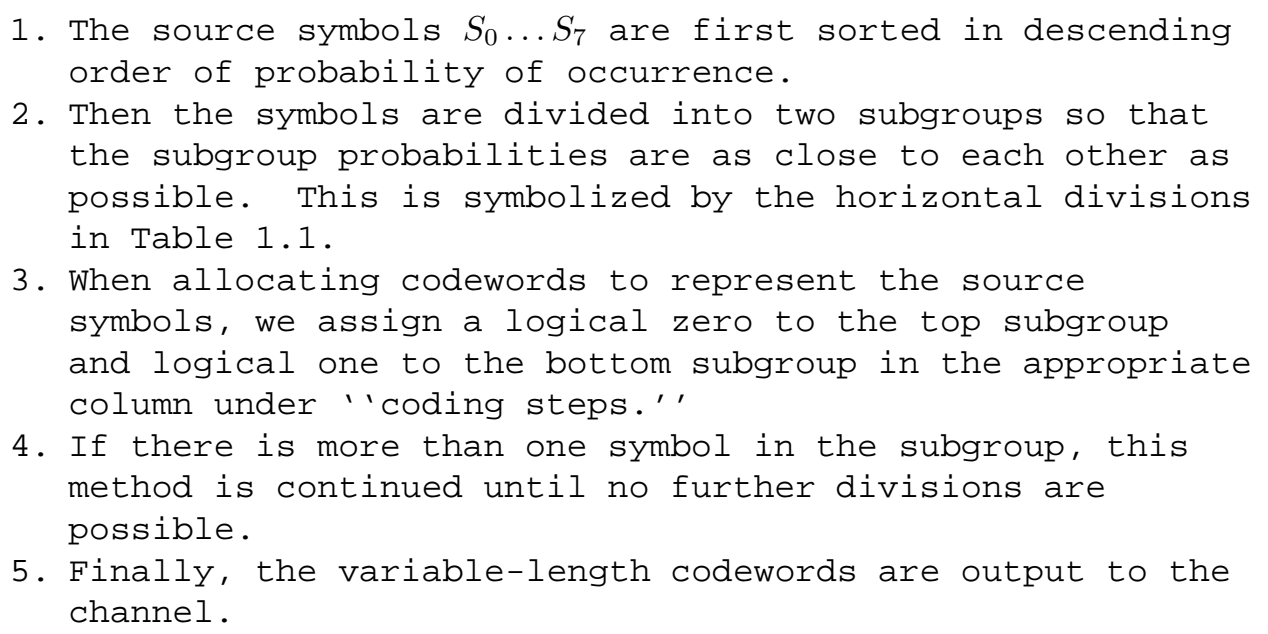

Coding efficiency can be defined as the ratio of the source information rate and the average output bit rate of the source encoder. If this ratio approaches unity, implying that the source encoder's output rate is close to the source information rate, the source encoder is highly efficient. There are many source encoding algorithms, but the most powerful approach suggested was Shannon's method [24], which is best illustrated by means of the following example, portrayed in Table 1.1, Algorithm 1, and Figure 1.6.

\subsubsection{Shannon-Fano Coding}

The Shannon-Fano coding algorithm is based on the simple concept of encoding frequent messages using short codewords and infrequent ones by long codewords, while reducing the average message length. This algorithm is part of virtually all treatises dealing with information theory, such as, for example, Carlson's work [31]. The formal coding steps listed in Algorithm 1 and in the flowchart of Figure 1.6 can be readily followed in the context of a simple example in Table 1.1. The average codeword length is given by weighting the length of any codeword by its probability, yielding:

$$
(0.27+0.2) \cdot 2+(0.17+0.16) \cdot 3+2 \cdot 0.06 \cdot 4+2 \cdot 0.04 \cdot 4 \approx 2.73 \text { [bit] }
$$




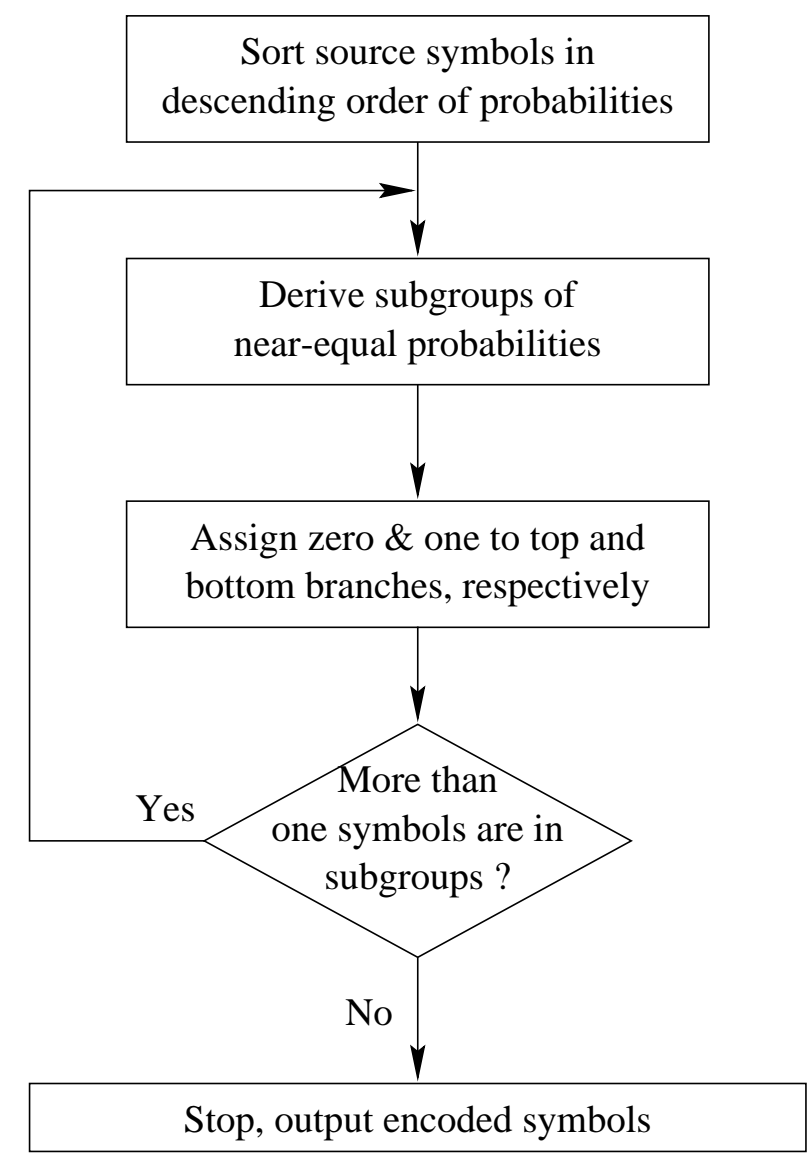

Figure 1.6: Shannon-Fano Coding Algorithm (see also Table 1.1 and Algorithm 1).

\begin{tabular}{|c|c|c|c|c|c|c|}
\hline Symb. & Prob. & \multicolumn{3}{|c|}{ Coding Steps } & Codeword \\
\cline { 3 - 5 } & & 1 & 2 & 3 & 4 & \\
\hline$S_{0}$ & 0.27 & 0 & 0 & & & 00 \\
$S_{1}$ & 0.20 & 0 & 1 & & & 01 \\
$S_{2}$ & 0.17 & 1 & 0 & 0 & & 100 \\
$S_{3}$ & 0.16 & 1 & 0 & 1 & & 101 \\
\cline { 4 - 5 }$S_{4}$ & 0.06 & 1 & 1 & 0 & 0 & 1100 \\
$S_{5}$ & 0.06 & 1 & 1 & 0 & 1 & 1101 \\
$S_{6}$ & 0.04 & 1 & 1 & 1 & 0 & 1110 \\
$S_{7}$ & 0.04 & 1 & 1 & 1 & 1 & 1111 \\
\hline
\end{tabular}

Table 1.1: Shannon-Fano Coding Example Based on Algorithm 1 and Figure 1.6 
Algorithm 2 (Huffman Coding) This algorithm summarizes the Huffman coding steps.

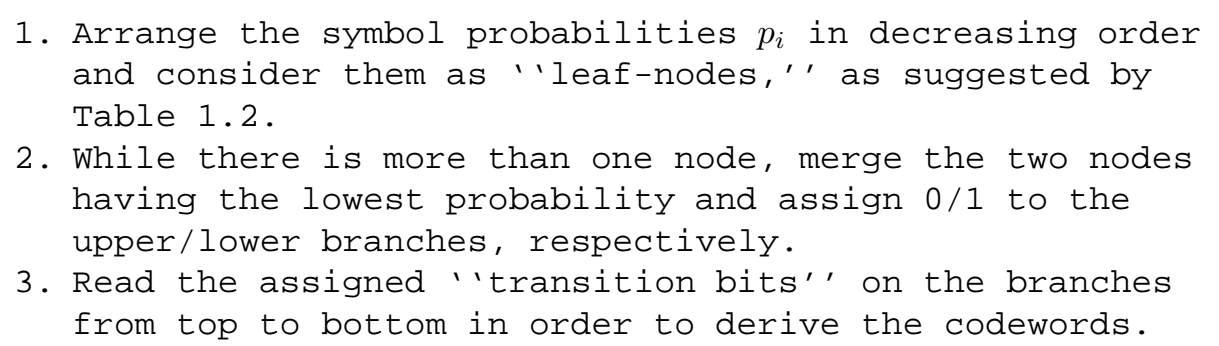

The entropy of the source is:

$$
\begin{aligned}
H= & -\sum_{i} p_{i} \log _{2} p_{i} \\
= & -\left(\log _{2} 10\right) \sum_{i} p_{i} \log _{10} p_{i} \\
\approx & -3.322 \cdot\left[0.27 \cdot \log _{10} 0.27+0.2 \cdot \log _{10} 0.2\right. \\
& +0.17 \cdot \log _{10} 0.17+0.16 \cdot \log _{10} 0.16 \\
& \left.+2 \cdot 0.06 \cdot \log _{10} 0.06+2 \cdot 0.04 \cdot \log _{10} 0.04\right] \\
\approx & 2.691[\mathrm{bit} / \text { symbol }] .
\end{aligned}
$$

Since the average codeword length of $2.73 \mathrm{bit} / \mathrm{symbol}$ is very close to the entropy of 2.691 bit/symbol, a high coding efficiency is predicted, which can be computed as:

$$
E \approx \frac{2.691}{2.73} \approx 98.6 \%
$$

The straightforward 3 bit/symbol binary coded decimal (BCD) assignment gives an efficiency of:

$$
E \approx \frac{2.691}{3} \approx 89.69 \% \text {. }
$$

In summary, Shannon-Fano coding allowed us to create a set of uniquely invertible mappings to a set of codewords, which facilitate a more efficient transmission of the source symbols, than straightforward BCD representations would. This was possible with no coding impairment (i.e., losslessly). Having highlighted the philosophy of the Shannon-Fano noiseless or distortionless coding technique, let us now concentrate on the closely related Huffman coding principle.

\subsubsection{Huffman Coding}

The Huffman Coding (HC) algorithm is best understood by referring to the flowchart of Figure 1.7 and to the formal coding description of Algorithm 2, while a simple practical example is portrayed in Table 1.2, which leads to the Huffman codes summarized in Table 1.3. Note that we used the same symbol probabilities as in our Shannon-Fano coding example, 


\begin{tabular}{|c|c|c|c|c|c|c|c|}
\hline \multirow[t]{2}{*}{ Symb. } & \multirow[t]{2}{*}{ Prob. } & \multicolumn{2}{|c|}{ Step $1 \& 2$} & \multicolumn{2}{|c|}{ Step 3 \& 4} & \multirow[t]{2}{*}{ Group } & \multirow[t]{2}{*}{ Code } \\
\hline & & Code & Prob. & Code & Prob. & & \\
\hline$S_{0}$ & 0.27 & & & & & $S_{0}$ & - \\
\hline$S_{1}$ & 0.20 & & & & & $S_{1}$ & - \\
\hline$\overline{S_{2}}$ & 0.17 & & & 0 & 0.33 & $S_{23}$ & 0 \\
\hline$\overline{S_{3}}$ & 0.16 & & & 1 & & & 1 \\
\hline$S_{4}$ & 0.06 & 0 & 0.12 & 0 & & & 00 \\
\hline$S_{5}$ & 0.06 & 1 & & 0 & 0.20 & $S_{4567}$ & 01 \\
\hline$S_{6}$ & 0.04 & 0 & 0.08 & 1 & & & 10 \\
\hline$S_{7}$ & 0.04 & 1 & & 1 & & & 11 \\
\hline
\end{tabular}

\begin{tabular}{|c|c|c|c|c|c|c|}
\hline \multirow[t]{2}{*}{ Symb. } & \multirow[t]{2}{*}{ Prob. } & \multicolumn{2}{|c|}{ Step 5\& 6} & \multicolumn{2}{|c|}{ Step 7} & \multirow[t]{2}{*}{ Codeword } \\
\hline & & Code & Prob. & Code & Prob. & \\
\hline$S_{23}$ & 0.33 & 0 & \multirow[t]{2}{*}{0.6} & \multirow[t]{2}{*}{0} & \multirow{4}{*}{1.0} & \multirow{4}{*}{$\begin{array}{l}00 \\
01 \\
10 \\
11\end{array}$} \\
\hline$S_{0}$ & 0.27 & 1 & & & & \\
\hline$S_{1}$ & 0.20 & $\overline{0}$ & \multirow[t]{2}{*}{0.4} & \multirow[t]{2}{*}{1} & & \\
\hline$S_{4567}$ & 0.20 & 1 & & & & \\
\hline
\end{tabular}

Table 1.2: Huffman Coding Example Based on Algorithm 2 and Figure 1.7 (for final code assignment see Table 1.3)

\begin{tabular}{|c|c|c|l|}
\hline Symbol & Probability & BCD & Huffman Code \\
\hline \hline$S_{0}$ & 0.27 & 000 & 01 \\
\hline$S_{1}$ & 0.20 & 001 & 10 \\
\hline$S_{2}$ & 0.17 & 010 & 000 \\
\hline$S_{3}$ & 0.16 & 011 & 001 \\
\hline$S_{4}$ & 0.06 & 100 & 1100 \\
\hline$S_{5}$ & 0.06 & 101 & 1101 \\
\hline$S_{6}$ & 0.04 & 110 & 1110 \\
\hline$S_{7}$ & 0.04 & 111 & 1111 \\
\hline
\end{tabular}

Table 1.3: Huffman Coding Example Summary of Table 1.2 


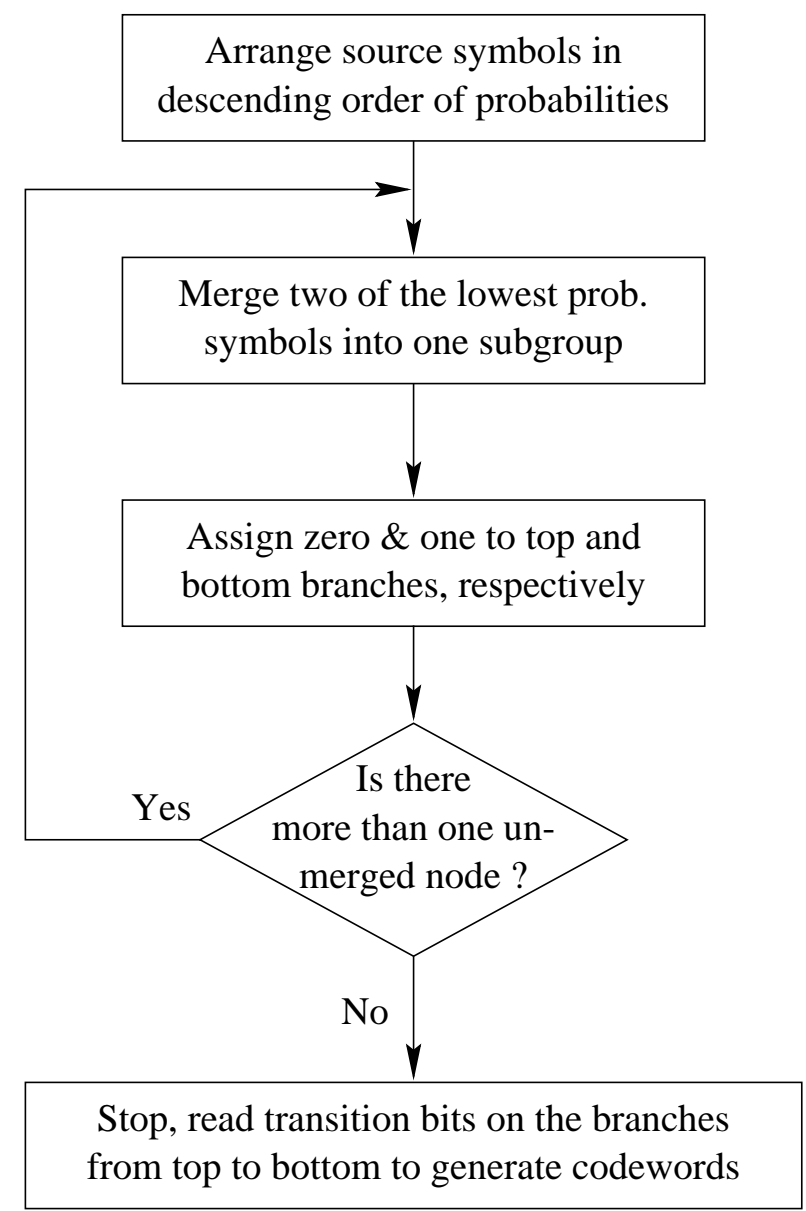

Figure 1.7: Huffman coding algorithm (see also Algorithm 2 and Table 1.2).

but the Huffman algorithm leads to a different codeword assignment. Nonetheless, the code's efficiency is identical to that of the Shannon-Fano algorithm.

The symbol-merging procedure can also be conveniently viewed using the example of Figure 1.8, where the Huffman codewords are derived by reading the associated 1 and 0 symbols from the end of the tree backward, that is, toward the source symbols $S_{0} \ldots S_{7}$. Again, these codewords are summarized in Table 1.3.

In order to arrive at a fixed average channel bit rate, which is convenient in many communications systems, a long buffer might be needed, causing storage and delay problems. Observe from Table 1.3 that the Huffman coding algorithm gives codewords that can be uniquely decoded, which is a crucial prerequisite for its practical employment. This is because no codeword can be a prefix of any longer one. For example, for the following sequence 


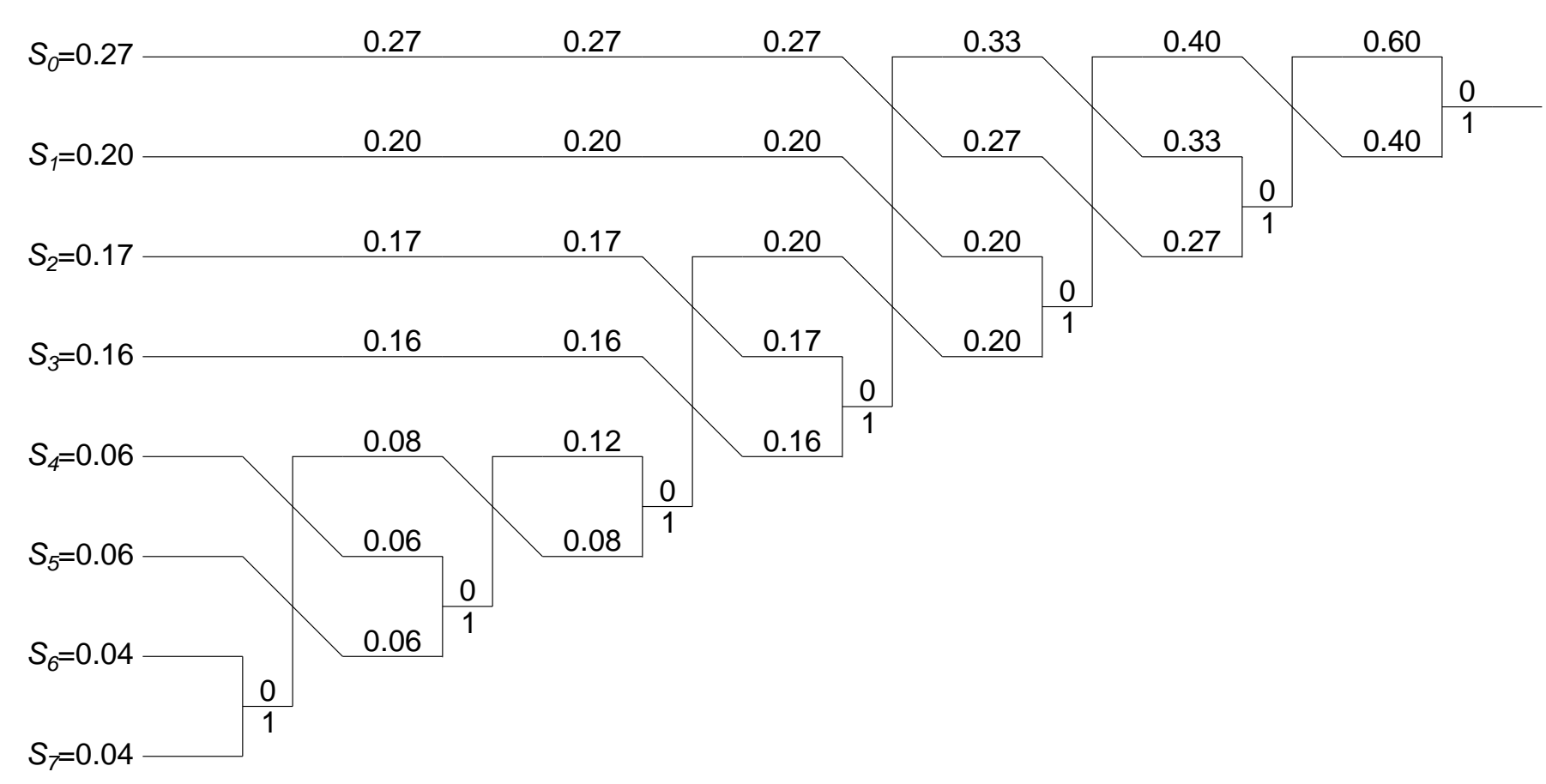


of codewords ..., 00, 10, 010, 110,1111, .. the source sequence of $\ldots S_{0}, S_{1}, S_{2}, S_{3}, S_{8} \ldots$ can be uniquely inferred from Table 1.3 .

In our discussions so far, we have assumed that the source symbols were completely independent of each other. Such a source is usually referred to as a memoryless source. By contrast, sources where the probability of a certain symbol also depends on what the previous symbol was are often termed sources exhibiting memory. These sources are typically bandlimited sample sequences, such as, for example, a set of correlated or "similar-magnitude" speech samples or adjacent video pixels. Let us now consider sources that exhibit memory.

\subsection{Entropy of Discrete Sources Exhibiting Memory}

Let us invoke Shannon's approach $[24-27,35,36]$ in order to illustrate sources with and without memory. Let us therefore consider an uncorrelated random white Gaussian noise (WGN) process, which was passed through a low-pass filter. The corresponding autocorrelation functions (ACF) and power spectral density (PSD) functions were portrayed in Figures 1.3 and 1.4. Observe in the figures that through low-pass filtering a WGN process introduces correlation by limiting the rate at which amplitude changes are possible, smoothing the amplitude of abrupt noise peaks. This example suggests that all bandlimited signals are correlated over a finite interval. Most analog source signals, such as speech and video, are inherently correlated, owing to physical restrictions imposed on the analog source. Hence all practical analog sources possess some grade of memory, a property that is also retained after sampling and quantization. An important feature of sources with memory is that they are predictable to a certain extent, hence, they can usually be more efficiently encoded than unpredictable sources having no memory.

\subsubsection{Two-State Markov Model for Discrete Sources Exhibiting Memory}

Let us now introduce a simple analytically tractable model for treating sources that exhibit memory. Predictable sources that have memory can be conveniently modeled by Markov processes. A source having a memory of one symbol interval directly "remembers" only the previously emitted source symbol and depending on this previous symbol it emits one of its legitimate symbols with a certain probability, which depends explicitly on the state associated with this previous symbol. A one-symbol-memory model is often referred to as a first-order model. For example, if in a first-order model the previous symbol can take only two different values, we have two different states, and this simple two-state first-order Markov model is characterized by the state transition diagram of Figure 1.9. Previously, in the context of Shannon-Fano and Huffman coding of memoryless information sources, we used the notation of $S_{i}, i=0,1, \ldots$ for the various symbols to be encoded. In this section, we are dealing with sources exhibiting memory and hence for the sake of distinction we use the symbol notation of $X_{i}, i=1,2, \ldots$. If, for the sake of illustration, the previous emitted symbol was $X_{1}$, the state machine of Figure 1.9 is in state $X_{1}$, and in the current signaling interval it can generate one of two symbols, namely, $X_{1}$ and $X_{2}$, whose probability depends explicitly on the previous state $X_{1}$. However, not all two-state Markov models are as simple as that of Figure 1.9, since the transitions from state $X_{1}$ to $X_{2}$ are not necessarily associated with emitting the same symbol as the transitions from state $X_{2}$ to $X_{1}$. Thus more elaborate example will be considered later in this chapter. 
Observe in Figure 1.9 that the corresponding transition probabilities from state $X_{1}$ are given by the conditional probabilities $p_{12}=P\left(X_{2} / X_{1}\right)$ and $p_{11}=P\left(X_{1} / X_{1}\right)=1-$ $P\left(X_{2} / X_{1}\right)$. Similar findings can be observed as regards state $X_{2}$. These dependencies can also be stated from a different point of view as follows. The probability of occurrence of a particular symbol depends not only on the symbol itself, but also on the previous symbol emitted. Thus, the symbol entropy for state $X_{1}$ and $X_{2}$ will now be characterized by means of the conditional probabilities associated with the transitions merging in these states. Explicitly, the symbol entropy for state $X_{i}, i=1,2$ is given by:

$$
\begin{aligned}
H_{i} & =\sum_{j=1}^{2} p_{i j} \cdot \log _{2} \frac{1}{p_{i j}} i=1,2 \\
& =p_{i 1} \cdot \log _{2} \frac{1}{p_{i 1}}+p_{i 2} \cdot \log _{2} \frac{1}{p_{i 2}}
\end{aligned}
$$

yielding the symbol entropies, that is, the average information carried by the symbols emitted in states $X_{1}$ and $X_{2}$, respectively, as:

$$
\begin{aligned}
& H_{1}=p_{11} \cdot \log _{2} \frac{1}{p_{11}}+p_{12} \cdot \log _{2} \frac{1}{p_{12}} \\
& H_{2}=p_{21} \cdot \log _{2} \frac{1}{p_{21}}+p_{22} \cdot \log _{2} \frac{1}{p_{22}}
\end{aligned}
$$

Both symbol entropies, $H_{1}$ and $H_{2}$, are characteristic of the average information conveyed by a symbol emitted in state $X_{1}$ and $X_{2}$, respectively. In order to compute the overall entropy $H$ of this source, they must be weighted by the probability of occurrence, $P_{1}$ and $P_{2}$, of these states:

$$
\begin{aligned}
H & =\sum_{i=1}^{2} P_{i} H_{i} \\
& =\sum_{i=1}^{2} P_{i} \sum_{j=1}^{2} p_{i j} \log _{2} \frac{1}{p_{i j}}
\end{aligned}
$$

Assuming a highly predictable source having high adjacent sample correlation, it is plausible that once the source is in a given state, it is more likely to remain in that state than to traverse into the other state. For example, assuming that the state machine of Figure 1.9 is in state $X_{1}$ and the source is a highly correlated, predictable source, we are likely to observe long runs of $X_{1}$. Conversely, once in state $X_{2}$, long strings of $X_{2}$ symbols will typically follow.

\subsubsection{N-State Markov Model for Discrete Sources Exhibiting Memory}

In general, assuming $N$ legitimate states, (i.e., $N$ possible source symbols) and following similar arguments, Markov models are characterised by their state probabilities $P\left(X_{i}\right), i=1 \ldots N$, where $N$ is the number of states, as well as by the transition probabilities $p_{i j}=P\left(X_{i} / X_{j}\right)$, 


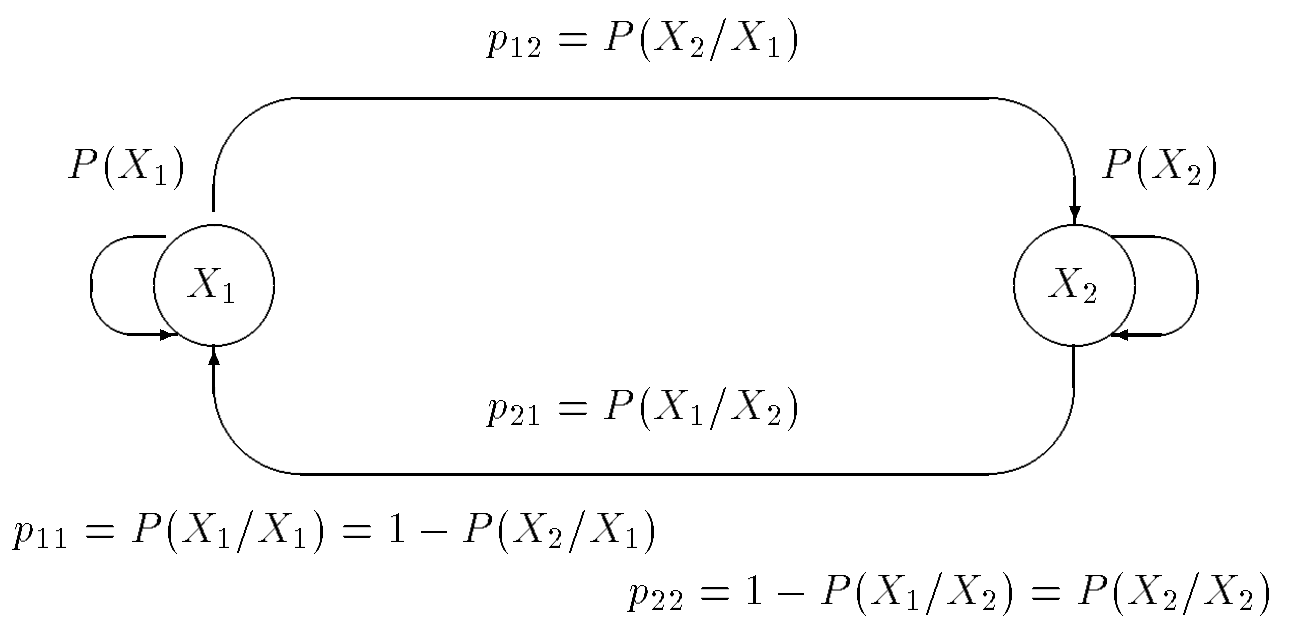

Figure 1.9: Two-state first-order Markov model.

where $p_{i j}$ explicitly indicates the probability of traversing from state $X_{j}$ to state $X_{i}$. Their further basic feature is that they emit a source symbol at every state transition, as will be shown in the context of an example presented in Section 1.7. Similarly to the two-state model, we define the entropy of a source having memory as the weighted average of the entropy of the individual symbols emitted from each state, where weighting is carried out taking into account the probability of occurrence of the individual states, namely $P_{i}$. In analytical terms, the symbol entropy for state $X_{i}, i=1 \ldots N$ is given by:

$$
H_{i}=\sum_{j=1}^{N} p_{i j} \cdot \log _{2} \frac{1}{p_{i j}} i=1 \ldots N .
$$

The averaged, weighted symbol entropies give the source entropy:

$$
\begin{aligned}
H & =\sum_{i=1}^{N} P_{i} H_{i} \\
& =\sum_{i=1}^{N} P_{i} \sum_{j=1}^{N} p_{i j} \log _{2} \frac{1}{p_{i j}} .
\end{aligned}
$$

Finally, assuming a source symbol rate of $v_{s}$, the average information emission rate $R$ of the source is given by:

$$
R=v_{s} \cdot H[\mathrm{bps}]
$$




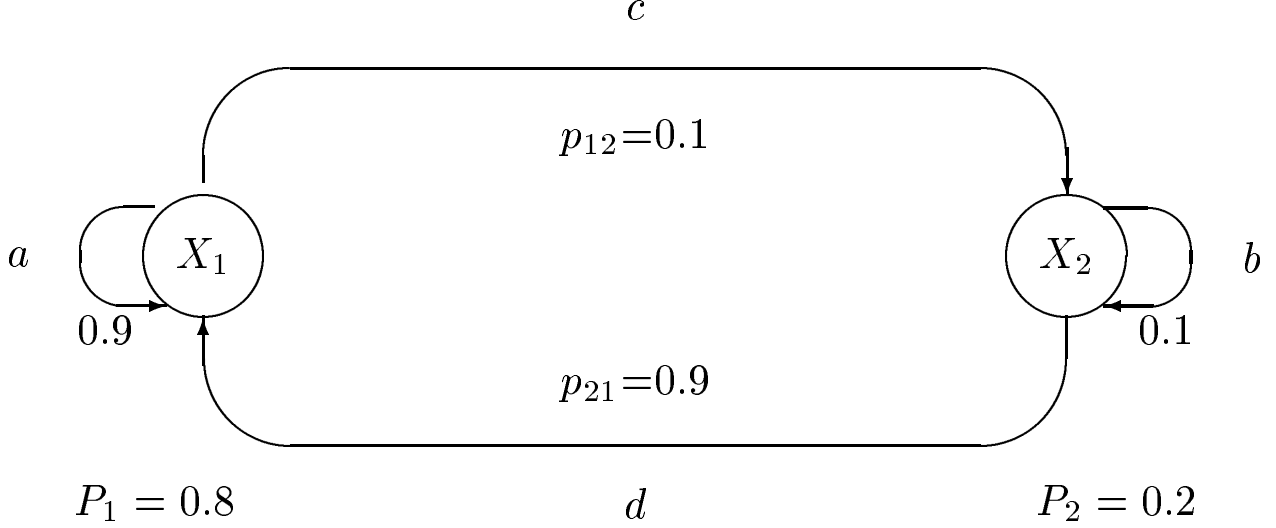

Figure 1.10: Two-state Markov model example.

\subsection{Examples}

\subsubsection{Two-State Markov Model Example}

As mentioned in the previous section, we now consider a slightly more sophisticated Markov model, where the symbols emitted upon traversing from state $X_{1}$ to $X_{2}$ are different from those when traversing from state $X_{2}$ to $X_{1}$. More explicitly:

- Consider a discrete source that was described by the two-state Markov model of Figure 1.9 , where the transition probabilities are

$$
\begin{array}{ll}
p_{11}=P\left(X_{1} / X_{1}\right)=0.9 & p_{22}=P\left(X_{2} / X_{2}\right)=0.1 \\
p_{12}=P\left(X_{1} / X_{2}\right)=0.1 & p_{21}=P\left(X_{2} / X_{1}\right)=0.9,
\end{array}
$$

while the state probabilities are

$$
P\left(X_{1}\right)=0.8 \text { and } P\left(X_{2}\right)=0.2 .
$$

The source emits one of four symbols, namely, $a, b, c$, and $d$, upon every state transition, as seen in Figure 1.10. Let us find

(a) the source entropy and

(b) the average information content per symbol in messages of one, two, and three symbols.

\section{- Message Probabilities}

Let us consider two sample sequences $a c b$ and $a a b$. As shown in Figure 1.10, the transitions leading to $a c b$ are $(1 \leadsto 1),(1 \leadsto 2)$, and $(2 \leadsto 2)$. The probability of encountering this sequence is $0.8 \cdot 0.9 \cdot 0.1 \cdot 0.1=0.0072$. The sequence $a a b$ has a probability of zero because the transition from $a$ to $b$ is illegal. Further path (i.e., message) probabilities are tabulated in Table 1.4 along with the information of $I=-\log _{2} P$ of all the legitimate messages. 


\begin{tabular}{|l|l|}
\hline Message Probabilities & $\begin{array}{c}\text { Information conveyed } \\
\text { (bit/message) }\end{array}$ \\
\hline$P_{a}=0.9 \times 0.8=0.72$ & $I_{a}=0.474$ \\
$P_{b}=0.1 \times 0.2=0.02$ & $I_{b}=5.644$ \\
$P_{c}=0.1 \times 0.8=0.08$ & $I_{c}=3.644$ \\
$P_{d}=0.9 \times 0.2=0.18$ & $I_{d}=2.474$ \\
\hline$P_{a a}=0.72 \times 0.9=0.648$ & $I_{a a}=0.626$ \\
$P_{a c}=0.72 \times 0.1=0.072$ & $I_{a c}=3.796$ \\
$P_{c b}=0.08 \times 0.1=0.008$ & $I_{c b}=6.966$ \\
$P_{c d}=0.08 \times 0.9=0.072$ & $I_{c d}=3.796$ \\
$P_{b b}=0.02 \times 0.1=0.002$ & $I_{b b}=8.966$ \\
$P_{b d}=0.02 \times 0.9=0.018$ & $I_{b d}=5.796$ \\
$P_{d a}=0.18 \times 0.9=0.162$ & $I_{d a}=2.626$ \\
$P_{d c}=0.18 \times 0.1=0.018$ & $I_{d c}=5.796$ \\
\hline
\end{tabular}

Table 1.4: Message Probabilities of Example

- Source Entropy

- According to Equation 1.27, the entropy of symbols $X_{1}$ and $X_{2}$ is computed as follows:

$$
\begin{aligned}
H_{1} & =-p_{12} \cdot \log _{2} p_{12}-p_{11} \cdot \log _{2} p_{11} \\
& =0.1 \cdot \log _{2} 10+0.9 \cdot \log _{2} \frac{1}{0.9} \\
& \approx 0.469 \mathrm{bit} / \mathrm{symbol} \\
H_{2} & =-p_{21} \cdot \log _{2} p_{21}-p_{22} \cdot \log _{2} p_{22} \\
& \approx 0.469 \mathrm{bit} / \mathrm{symbol}
\end{aligned}
$$

- Then their weighted average is calculated using the probability of occurrence of each state in order to derive the average information per message for this source:

$$
H \approx 0.8 \cdot 0.469+0.2 \cdot 0.469 \approx 0.469 \mathrm{bit} / \mathrm{symbol} .
$$

- The average information per symbol $I_{2}$ in two-symbol messages is computed from the entropy $h_{2}$ of the two-symbol messages as follows:

$$
\begin{aligned}
h_{2} & =\sum_{1}^{8} P_{\text {symbol }} \cdot I_{\text {symbol }} \\
& =P_{a a} \cdot I_{a a}+P_{a c} \cdot I_{a c}+\ldots+P_{d c} \cdot I_{d c} \\
& \approx 1.66 \mathrm{bits} / 2 \mathrm{symbols},
\end{aligned}
$$

giving $I_{2}=h_{2} / 2 \approx 0.83$ bits/symbol information on average upon receiving a two-symbol message. 
- There are eight two-symbol messages; hence, the maximum possible information conveyed is $\log _{2} 8=3$ bits $/ 2$ symbols, or 1.5 bits/symbol. However, since the symbol probabilities of $P_{1}=0.8$ and $P_{2}=0.2$ are fairly different, this scheme has a significantly lower conveyed information per symbol, namely, $I_{2} \approx 0.83$ bits/symbol.

- Similarly, one can find the average information content per symbol for arbitrarily long messages of concatenated source symbols. For one-symbol messages we have:

$$
\begin{aligned}
I_{1}=h_{1} & =\sum_{1}^{4} P_{\text {symbol }} \cdot I_{\text {symbol }} \\
& =P_{a} \cdot I_{a}+\ldots+P_{d} \cdot I_{d} \\
& \approx 0.72 \times 0.474+\ldots+0.18 \times 2.474 \\
& \approx 0.341+0.113+0.292+0.445 \\
& \approx 1.191 \mathrm{bit} / \mathrm{symbol} .
\end{aligned}
$$

We note that the maximum possible information carried by one-symbol messages is $h_{1 \max }=\log _{2} 4=2 \mathrm{bit} /$ symbol, since there are four one-symbol messages in Table 1.4.

- Observe the important tendency, in which, when sending longer messages of dependent sources, the average information content per symbol is reduced. This is due to the source's memory, since consecutive symbol emissions are dependent on previous ones and hence do not carry as much information as independent source symbols. This becomes explicit by comparing $I_{1} \approx 1.191$ and $I_{2} \approx 0.83$ bits/symbol.

- Therefore, expanding the message length to be encoded yields more efficient coding schemes, requiring a lower number of bits, if the source has a memory. This is the essence of Shannon's source coding theorem.

\subsubsection{Four-State Markov Model for a 2-Bit Quantizer}

Let us now augment the previously introduced two-state Markov-model concepts with the aid of a four-state example. Let us assume that we have a discrete source constituted by a 2-bit quantizer, which is characterized by Figure 1.11. Assume further that due to bandlimitation only transitions to adjacent quantization intervals are possible, since the bandlimitation restricts the input signal's rate of change. The probability of the signal samples residing in intervals $1-4$ is given by:

$$
P(1)=P(4)=0.1, \quad P(2)=P(3)=0.4 .
$$

The associated state transition probabilities are shown in Figure 1.11, along with the quantized samples $a, b, c$, and $d$, which are transmitted when a state transition takes place, that is, when taking a new sample from the analog source signal at the sampling-rate $f_{s}$.

Although we have stipulated a number of simplifying assumptions, this example attempts to illustrate the construction of Markov models in the context of a simple practical problem. 


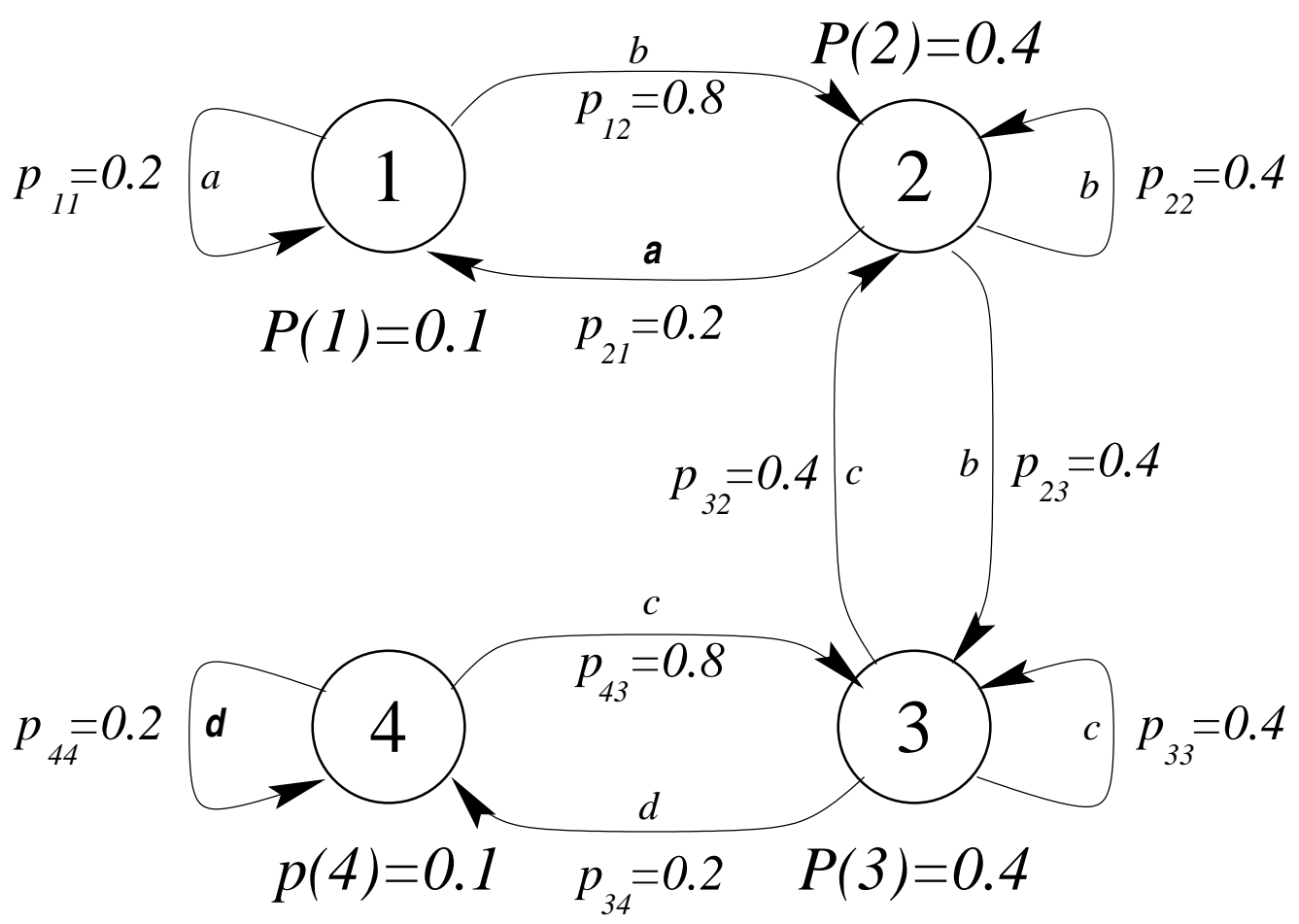

Figure 1.11: Four-state Markov model for a 2-bit quantizer.

Next we construct a simpler example for augmenting the underlying concepts and set aside the above four-state Markov-model example as a potential exercise for the reader.

\subsection{Generating Model Sources}

\subsubsection{Autoregressive Model}

In evaluating the performance of information processing systems, such as encoders and predictors, it is necessary to have "standardized" or easily described model sources. Although a set of semistandardized speech and images test sequences is widely used by researchers in codec performance testing, in contrast to analytical model sources, real speech or image sources cannot be used in analytical studies. A widely used analytical model source is the Autoregressive $(A R)$ model. A zero mean random sequence $y(n)$ is called an AR process of $\operatorname{order} p$, if it is generated as follows:

$$
y(n)=\sum_{k=1}^{p} a_{k} y(n-k)+\varepsilon(n), \forall n,
$$


where $\varepsilon(n)$ is an uncorrelated zero-mean, random input sequence with variance $\sigma^{2}$; that is,

$$
\begin{array}{r}
E\{\varepsilon(n)\}=0 \\
E\left\{\varepsilon^{2}(n)\right\}=\sigma^{2} \\
E\{\varepsilon(n) \cdot y(m)\}=0 .
\end{array}
$$

From Equation 1.35 we surmise that an AR system recursively generates the present output from $p$ number of previous output samples given by $y(n-k)$ and the present random input sample $\varepsilon(n)$.

\subsubsection{AR Model Properties}

AR models are very useful in studying information processing systems, such as speech and image codecs, predictors, and quantizers. They have the following basic properties:

1. The first term of Equation 1.35, which is repeated here for convenience,

$$
\hat{y}(n)=\sum_{k=1}^{p} a_{k} y(n-k)
$$

defines a predictor, giving an estimate $\hat{y}(n)$ of $y(n)$, which is associated with the minimum mean squared error between the two quantities.

2. Although $\hat{y}(n)$ and $y(n)$ depend explicitly only on the past $p$ number of samples of $y(n)$, through the recursive relationship of Equation 1.35 this entails the entire past of $y(n)$. This is because each of the previous $p$ samples depends on their predecessors.

3. Then Equation 1.35 can be written in the form of:

$$
y(n)=\hat{y}(n)+\varepsilon(n),
$$

where $\varepsilon(n)$ is the prediction error and $\hat{y}(n)$ is the minimum variance prediction estimate of $y(n)$.

4. Without proof, we state that for a random Gaussian distributed prediction error sequence $\varepsilon(n)$ these properties are characteristic of a $p^{\text {th }}$ order Markov process portrayed in Figure 1.12. When this model is simplified for the case of $p=1$, we arrive at the schematic diagram shown in Figure 1.13.

5. The power spectral density (PSD) of the prediction error sequence $\varepsilon(n)$ is that of a random "white-noise" sequence, containing all possible frequency components with the same energy. Hence, its autocorrelation function (ACF) is the Kronecker delta function, given by the Wiener-Khintchine theorem:

$$
E\{\varepsilon(n) \cdot \varepsilon(m)\}=\sigma^{2} \delta(n-m) .
$$

\subsubsection{First-Order Markov Model}

A variety of practical information sources are adequately modeled by the analytically tractable first-order Markov model depicted in Figure 1.13, where the prediction order is $p=1$. With 


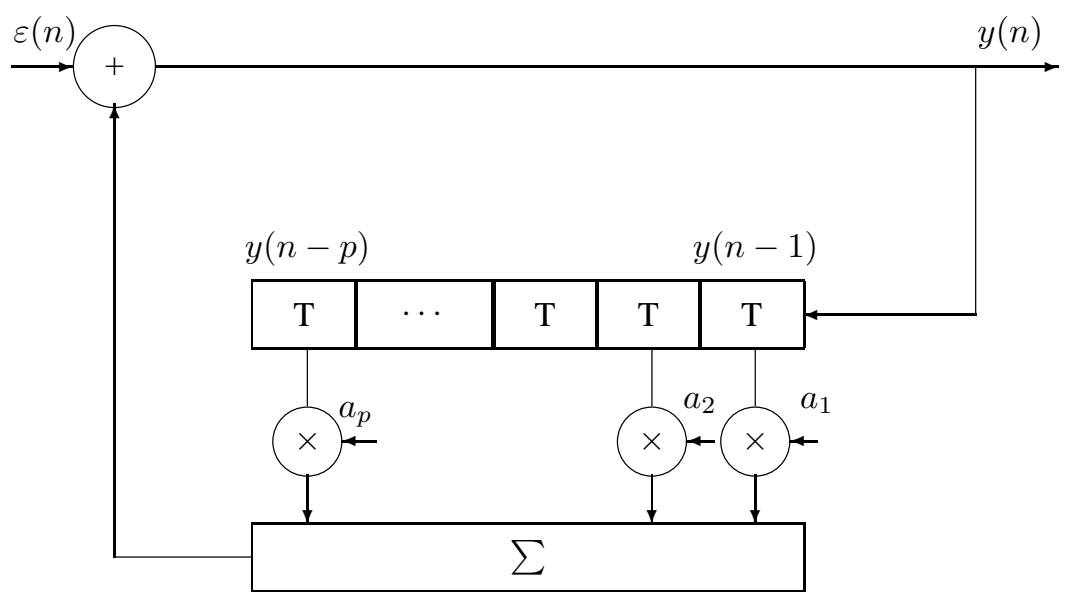

Figure 1.12: Markov model of order $p$.

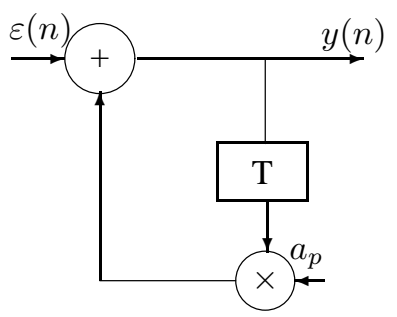

Figure 1.13: First-order Markov model.

the aid of Equation 1.35 we have

$$
y(n)=\varepsilon(n)+a y(n-1),
$$

where $a$ is the adjacent sample correlation of the process $y(n)$. Using the following recursion:

$$
\begin{aligned}
& y(n-1)=\varepsilon(n-1)+a_{1} y(n-2) \\
& \vdots \quad \vdots \quad \vdots \\
& y(n-k)=\varepsilon(n-k)+a_{1} y(n-k-1)
\end{aligned}
$$




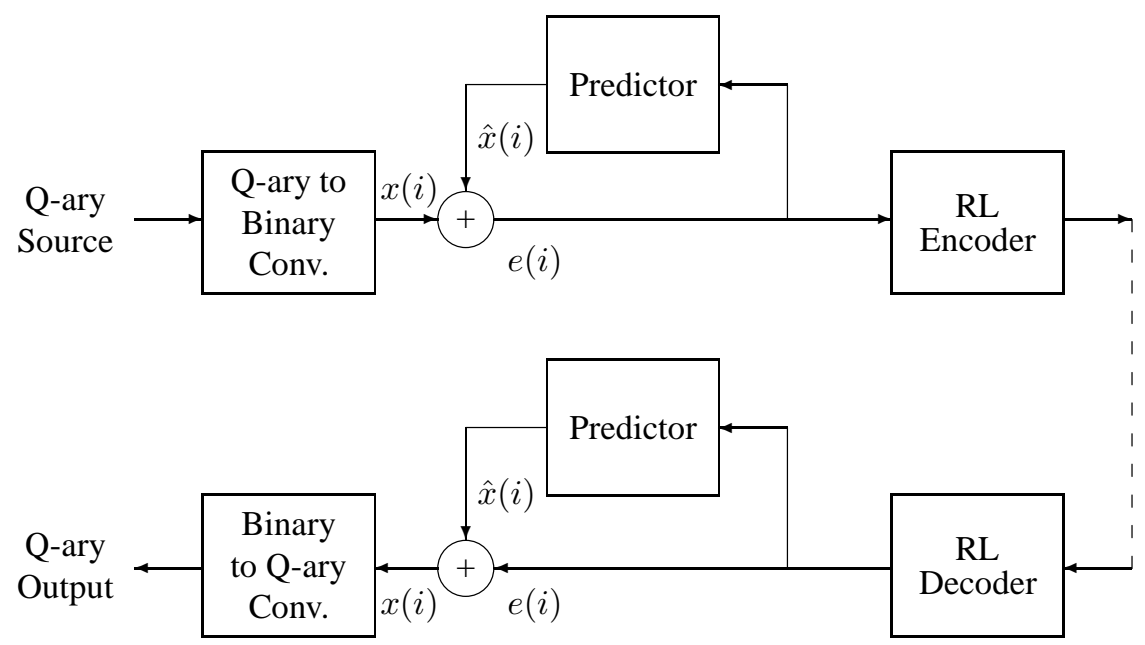

Figure 1.14: Predictive run-length codec scheme. (C)Carlson [31].

we arrive at:

$$
\begin{aligned}
y(n) & =\varepsilon(n)+a_{1}[\varepsilon(n-1)+a y(n-2)] \\
& =\varepsilon(n)+a_{1} \varepsilon(n-1)+a^{2} y(n-2),
\end{aligned}
$$

which can be generalized to:

$$
y(n)=\sum_{j=0}^{\infty} a^{j} \varepsilon(n-j) .
$$

Clearly, Equation 1.40 describes the first-order Markov process by the help of the adjacent sample correlation $a_{1}$ and the uncorrelated zero-mean random Gaussian process $\varepsilon(n)$.

\subsection{Run-Length Coding for Discrete Sources Exhibiting Memory}

\subsubsection{Run-Length Coding Principle [31]}

For discrete sources having memory, (i.e., possessing intersample correlation), the coding efficiency can be significantly improved by predictive coding, allowing the required transmission rate and hence the channel bandwidth to be reduced. Particularly amenable to run-length coding are binary sources with inherent memory, such as black and white documents, where the predominance of white pixels suggests that a Run-Length-Coding (RLC) scheme, which encodes the length of zero runs, rather than repeating long strings of zeros, provides high coding efficiency.

Following Carlson's interpretation [31], a predictive RLC scheme can be constructed according to Figure 1.14. The q-ary source messages are first converted to binary bit format. 


\begin{tabular}{|c|c|c|}
\hline $\begin{array}{c}\text { Length of 0-run } \\
1\end{array}$ & $\begin{array}{c}\text { Encoder Output } \\
\text { (n-bit codeword) }\end{array}$ & Decoder Output \\
\hline 0 & $00 \cdots 000$ & 1 \\
1 & $00 \cdots 001$ & 01 \\
2 & $00 \cdots 010$ & 001 \\
3 & $00 \cdots 011$ & 0001 \\
$\vdots$ & $\vdots$ & $\vdots$ \\
$N-1$ & $11 \cdots 110$ & $00 \cdots 01$ \\
$\geq N=2^{n}-1$ & $11 \cdots 111$ & $00 \cdots 00$ \\
\hline
\end{tabular}

Table 1.5: Run-length Coding Table (C) Carlson, 1975 [31]

For example, if an 8-bit analog-digital converter (ADC) is used, the 8-bit digital samples are converted to binary format. This bit-stream, $x(i)$, is then compared with the output signal of the predictor, $\hat{x}(i)$, which is fed with the prediction error signal $e(i)$. The comparator is a simple mod-2 gate, outputting a logical 1, whenever the prediction fails; that is, the predictor's output is different from the incoming bit $x(i)$. If, however, $x(i)=\hat{x}(i)$, the comparator indicates this by outputting a logical 0 . For highly correlated signals from sources with significant memory the predictions are usually correct, and hence long strings of 0 runs are emitted, interspersed with an occasional 1. Thus, the prediction error signal $e(i)$ can be efficiently run-length encoded by noting and transmitting the length of zero runs.

The corresponding binary run-length coding principle becomes explicit from Table 1.5 and from our forthcoming coding efficiency analysis.

\subsubsection{Run-Length Coding Compression Ratio [37]}

Following Jain's interpretation [37], let us now investigate the RLC efficiency by assuming that a run of $r$ successive logical 0 s is followed by a 1 . Instead of directly transmitting these strings, we represent such a string as an $n$-bit word giving the length of the 0-run between successive logical ones. When a 0 -run longer than $N=2^{n}-1$ bits occurs, this is signaled as the all 1 codeword, informing the decoder to wait for the next RLC codeword before releasing the decoded sequence. Again, the scheme's operation is characterized by Table 1.5. Clearly, data compression is achieved if the average number of 0 data bits per run $d$ is higher than the number of bits, $n$, required to encode the 0-run length. Let us therefore compute the average number of bits per run without RLC. If a run of $r$ logical zeros are followed by a 1, the runlength is $(r+1)$. The expected or mean value of $(r+1)$, namely, $d=\overline{(r+1)}$, is calculated by weighting each specific $(r+1)$ with its probability of occurrence that is, with its discrete PDF $c(r)$ and then averaging the weighted components, in:

$$
d=\overline{(r+1)}=\sum_{r=0}^{N-1}(r+1) \cdot c(r)+N c(N) .
$$



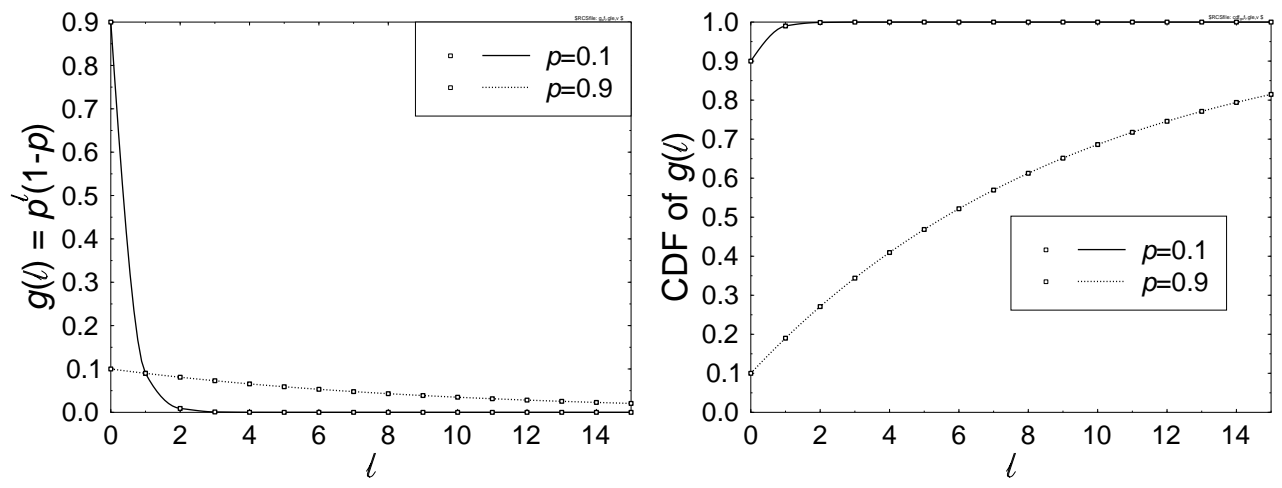

Figure 1.15: CDF and PDF of the geometric distribution of run-length $l$.

The PDF of a run of $r$ zeros followed by a 1 is given by:

$$
c(r)= \begin{cases}p^{r}(1-p) & 0 \leq r \leq N-1 \\ p^{N} & r=N,\end{cases}
$$

since the probability of $N$ consecutive zeros is $p^{N}$ if $r=N$, while for shorter runs the joint probability of $r$ zeros followed by a 1 is given by $p^{r} \cdot(1-p)$. The PDF and CDF of this distribution are shown in Figure 1.15 for $p=0.9$ and $p=0.1$, where $p$ represents the probability of a logical zero bit. Substituting Equation 1.42 in Equation 1.41 gives:

$$
\begin{aligned}
d & =N \cdot p^{N}+\sum_{r=0}^{N-1}(r+1) \cdot p^{r} \cdot(1-p) \\
& =N \cdot p^{N}+1 \cdot p^{0} \cdot(1-p)+2 \cdot p \cdot(1-p)+\ldots+N \cdot p^{N-1} \cdot(1-p) \\
& =N \cdot p^{N}+1+2 p+3 p^{2}+\ldots+N \cdot p^{N-1}-p-2 p^{2} \ldots-N \cdot p^{N} \\
& =1+p+p^{2}+\cdots p^{N-1} .
\end{aligned}
$$

Equation 1.43 is a simple geometric progression, given in closed form as:

$$
d=\frac{1-p^{N}}{1-p}
$$

RLC Example: Using a run-length coding memory of $M=31$ and a zero symbol probability of $p=0.95$, characterize the RLC efficiency.

Substituting $N$ and $p$ into Equation 1.44 for the average run-length we have:

$$
d=\frac{1-0.95^{31}}{1-0.95} \approx \frac{1-0.204}{0.05} \approx 15.92
$$


The compression ratio $C$ achieved by RLC is given by:

$$
C=\frac{d}{n}=\frac{1-p^{N}}{n(1-p)} \approx \frac{15.92}{5} \approx 3.18 .
$$

The achieved average bit rate is

$$
B=\frac{n}{d} \approx 0.314 \mathrm{bit} / \mathrm{pixel},
$$

and the coding efficiency is computed as the ratio of the entropy (i.e., the lowest possible bit rate and the actual bit rate). The source entropy is given by:

$$
\begin{aligned}
H & \approx-0.95 \cdot 3.322 \cdot \log _{10} 0.95-0.05 \cdot 3.322 \cdot \log _{10} 0.05 \\
& \approx 0.286 \mathrm{bit} / \mathrm{symbol},
\end{aligned}
$$

giving a coding efficiency of:

$$
E=H / B \approx 0.286 / 0.314 \approx 91 \% .
$$

This concludes our RLC example.

\subsection{Information Transmission via Discrete Channels}

Let us now return to Shannon's classic references [24-27, 35, 36] and assume that both the channel and the source are discrete, and let us evaluate the amount of information transmitted via the channel. We define the channel capacity characterizing the channel and show that according to Shannon nearly error-free information transmission is possible at rates below the channel capacity via the binary symmetric channel (BSC). Let us begin our discourse with a simple introductory example.

\subsubsection{Binary Symmetric Channel Example}

Let us assume that a binary source is emitting a logical 1 with a probability of $P(1)=$ 0.7 and a logical 0 with a probability of $P(0)=0.3$. The channel's error probability is $p_{e}=0.02$. This scenario is characterized by the binary symmetric channel (BSC) model of Figure 1.16. The probability of error-free reception is given by that of receiving 1 , when a logical 1 is transmitted plus the probability of receiving a 0 when 0 is transmitted, which is also plausible from Figure 1.16. For example, the first of these two component probabilities can be computed with the aid of Figure 1.16 as the product of the probability $P(1)$ of a logical 1 being transmitted and the conditional probability $P(1 / 1)$ of receiving a 1 , given the condition that a 1 was transmitted:

$$
\begin{gathered}
P\left(Y_{1}, X_{1}\right)=P\left(X_{1}\right) \cdot P\left(Y_{1} / X_{1}\right) \\
P(1,1)=P(1) \cdot P(1 / 1)=0.7 \cdot 0.98=0.686 .
\end{gathered}
$$




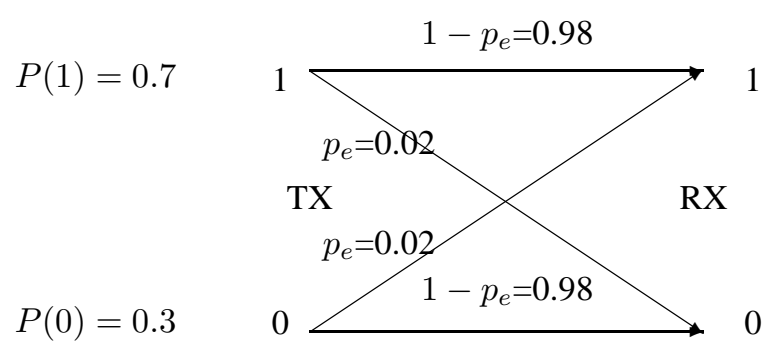

Figure 1.16: The binary symmetric channel. (C)Shannon [26], BSTJ, 1948.

Similarly, the probability of the error-free reception of a logical 0 is given by:

$$
\begin{aligned}
P\left(Y_{0}, X_{0}\right) & =P\left(X_{0}\right) \cdot P\left(Y_{0} / X_{0}\right) \\
P(0,0) & =P(0) \cdot P(0 / 0)=0.3 \cdot 0.98=0.294
\end{aligned}
$$

giving the total probability of error-free reception as:

$$
P_{\text {correct }}=P(1,1)+P(0,0)=0.98 \text {. }
$$

Following similar arguments, the probability of erroneous reception is also given by two components. For example, using Figure 1.16, the probability of receiving a 1 when a 0 was transmitted is computed by multiplying the probability $P(0)$ of a logical 0 being transmitted by the conditional probability $P(1 / 0)$ of receiving a logical 1 , given the fact that a 0 is known to have been transmitted:

$$
\begin{aligned}
P\left(Y_{1}, X_{0}\right) & =P\left(X_{0}\right) \cdot P\left(Y_{1} / X_{0}\right) \\
P(1,0) & =P(0) \cdot P(1 / 0)=0.3 \cdot 0.02=0.006 .
\end{aligned}
$$

Conversely,

$$
\begin{aligned}
P\left(Y_{0}, X_{1}\right) & =P\left(X_{1}\right) \cdot P\left(Y_{0} / X_{1}\right) \\
P(0,1) & =P(1) \cdot P(0 / 1)=0.7 \cdot 0.02=0.014
\end{aligned}
$$

yielding a total error probability of:

$$
P_{\text {error }}=P(1,0)+P(0,1)=0.02 \text {, }
$$

which is constituted by the above two possible error events. 
Viewing events from a different angle, we observe that the total probability of receiving $l$ is that of receiving a transmitted 1 correctly plus a transmitted 0 incorrectly:

$$
\begin{aligned}
P_{1} & =P(1) \cdot\left(1-p_{e}\right)+P(0) \cdot p_{e} \\
& =0.7 \cdot 0.98+0.3 \cdot 0.02=0.686+0.006=0.692 .
\end{aligned}
$$

On the same note, the probability of receiving 0 is that of receiving a transmitted 0 correctly plus a transmitted 1 incorrectly:

$$
\begin{aligned}
P_{0} & =P(0) \cdot\left(1-p_{e}\right)+P(1) \cdot p_{e} \\
& =0.3 \cdot 0.98+0.7 \cdot 0.02=0.294+0.014=0.308 .
\end{aligned}
$$

In the next example, we further study the performance of the BSC for a range of different parameters in order to gain a deeper insight into its behavior.

Example: Repeat the above calculations for $P(1)=1,0.9,0.5$, and $p_{e}=0,0.1,0.2,0.5$ using the BSC model of Figure 1.16. Compute and tabulate the probabilities $P(1,1)$, $P(0,0), P(1,0), P(0,1), P_{\text {correct }}, P_{\text {error }}, P_{1}$, and $P_{0}$ for these parameter combinations, including also their values for the previous example, namely, for $P(1)=0.7$, $P(0)=0.3$ and $p_{e}=0.02$. Here we neglected the details of the calculations and summarized the results in Table 1.6. Some of the above quantities are plotted for further study in Figure 1.17, which reveals the interdependency of the various probabilities for the interested reader.

Having studied the performance of the BSC, the next question that arises is, how much information can be inferred upon reception of a 1 and a 0 over an imperfect (i.e., error-prone) channel. In order to answer this question, let us first generalize the above intuitive findings in the form of Bayes' rule.

\subsubsection{Bayes' Rule}

Let $Y_{j}$ represent the received symbols and $X_{i}$ the transmitted symbols having probabilities of $P\left(Y_{j}\right)$ and $P\left(X_{i}\right)$, respectively. Let us also characterize the forward transition probabilities of the binary symmetric channel as suggested by Figure 1.18 .

Then in general, following from the previous introductory example, the joint probability $P\left(Y_{j}, X_{i}\right)$ of receiving $Y_{j}$, when the transmitted source symbol was $X_{i}$, is computed as the probability $P\left(X_{i}\right)$ of transmitting $X_{i}$, multiplied by the conditional probability $P\left(Y_{j} / X_{i}\right)$ of receiving $Y_{j}$, when $X_{i}$ is known to have been transmitted:

$$
P\left(Y_{j}, X_{i}\right)=P\left(X_{i}\right) \cdot P\left(Y_{j} / X_{i}\right)
$$

a result that we have already intuitively exploited in the previous example. Since for the joint probabilities $P\left(Y_{j}, X_{i}\right)=P\left(X_{i}, Y_{j}\right)$ holds, we have:

$$
\begin{aligned}
P\left(X_{i}, Y_{j}\right) & =P\left(Y_{j}\right) \cdot P\left(X_{i} / Y_{j}\right) \\
& =P\left(X_{i}\right) \cdot P\left(Y_{j} / X_{i}\right)
\end{aligned}
$$




\begin{tabular}{|c|c|c|c|c|c|c|c|c|c|c|}
\hline$p_{e}$ & $P(1)$ & $P(0)$ & $\begin{aligned} & \left(1-p_{e}\right) \\
= & P(1 / 1) \\
= & P(0 / 0)\end{aligned}$ & $P(1,1)$ & $P(0,0)$ & $\begin{aligned} & p_{e} \\
= & P(1 / 0) \\
= & P(0 / 1)\end{aligned}$ & $P(1,0)$ & $P(0,1)$ & $P_{1}$ & $P_{0}$ \\
\hline \multirow{4}{*}{0} & 1 & 0 & 1 & 1 & 0 & 0 & 0 & 0 & 1 & 0 \\
\hline & 0.9 & 0.1 & 1 & 0.9 & 0.1 & 0 & 0 & 0 & 0.9 & 0.1 \\
\hline & 0.7 & 0.3 & 1 & 0.7 & 0.3 & 0 & 0 & 0 & 0.7 & 0.3 \\
\hline & 0.5 & 0.5 & 1 & 0.5 & 0.5 & 0 & 0 & 0 & 0.5 & 0.5 \\
\hline \multirow{4}{*}{0.02} & 1 & 0 & 0.98 & 0.98 & 0 & 0.02 & 0 & 0.02 & 0.98 & 0.02 \\
\hline & 0.9 & 0.1 & 0.98 & 0.882 & 0.098 & 0.02 & 0.002 & 0.018 & 0.884 & 0.116 \\
\hline & 0.7 & 0.3 & 0.98 & 0.686 & 0.294 & 0.02 & 0.006 & 0.014 & 0.692 & 0.308 \\
\hline & 0.5 & 0.5 & 0.98 & 0.49 & 0.49 & 0.02 & 0.01 & 0.01 & 0.491 & 0.509 \\
\hline \multirow{4}{*}{0.1} & 1 & 0 & 0.9 & 0.9 & 0 & 0.1 & 0 & 0.1 & 0.9 & 0.1 \\
\hline & 0.9 & 0.1 & 0.9 & 0.81 & 0.09 & 0.1 & 0.01 & 0.09 & 0.811 & 0.189 \\
\hline & 0.7 & 0.3 & 0.9 & 0.63 & 0.27 & 0.1 & 0.03 & 0.07 & 0.723 & 0.277 \\
\hline & 0.5 & 0.5 & 0.9 & 0.45 & 0.45 & 0.1 & 0.05 & 0.05 & 0.455 & 0.545 \\
\hline \multirow{4}{*}{0.2} & 1 & 0 & 0.8 & 0.8 & 0 & 0.2 & 0 & 0.2 & 0.8 & 0.2 \\
\hline & 0.9 & 0.1 & 0.8 & 0.72 & 0.08 & 0.2 & 0.02 & 0.18 & 0.722 & 0.278 \\
\hline & 0.7 & 0.3 & 0.8 & 0.56 & 0.24 & 0.2 & 0.06 & 0.14 & 0.566 & 0.434 \\
\hline & 0.5 & 0.5 & 0.8 & 0.40 & 0.40 & 0.2 & 0.1 & 0.1 & 0.5 & 0.5 \\
\hline \multirow{4}{*}{0.5} & 1 & 0 & 0.5 & 0.5 & 0 & 0.5 & 0 & 0.5 & 0.5 & 0.5 \\
\hline & 0.9 & 0.1 & 0.5 & 0.45 & 0.05 & 0.5 & 0.05 & 0.45 & 0.5 & 0.5 \\
\hline & 0.7 & 0.3 & 0.5 & 0.35 & 0.15 & 0.5 & 0.15 & 0.35 & 0.5 & 0.5 \\
\hline & 0.5 & 0.5 & 0.5 & 0.25 & 0.25 & 0.5 & 0.25 & 0.25 & 0.5 & 0.5 \\
\hline
\end{tabular}



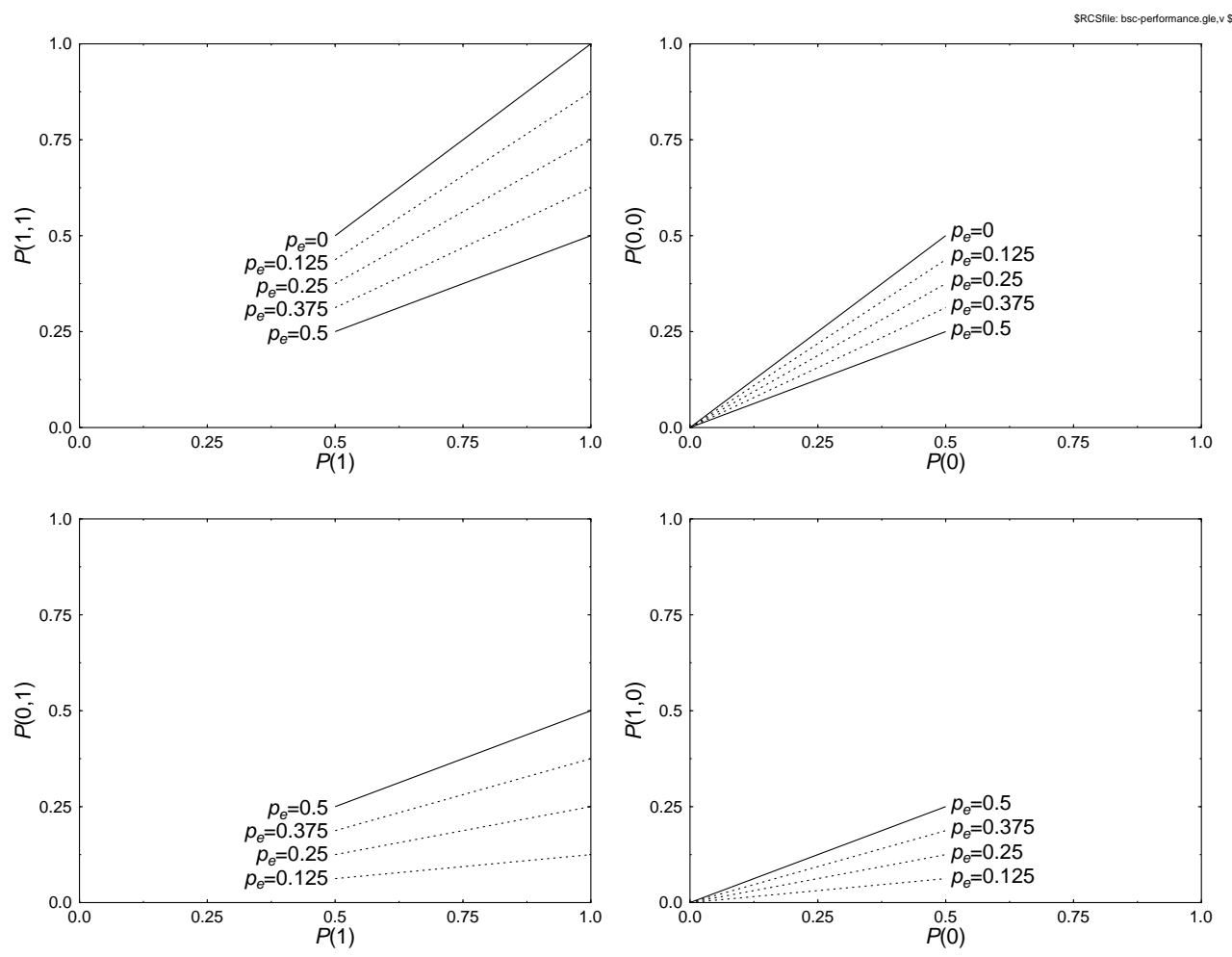

Figure 1.17: BSC performance for $p_{e}=0,0.125,0.25,0.375$, and 0.5 .

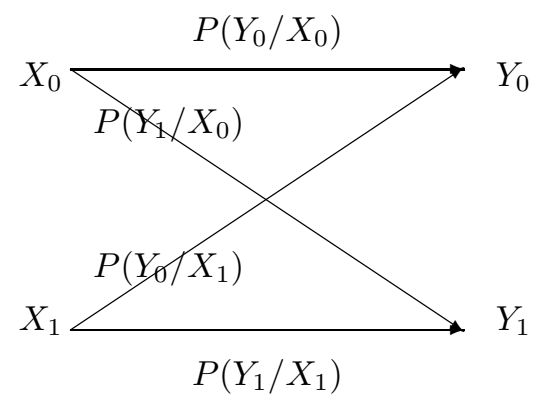

Figure 1.18: Forward transition probabilities of the nonideal binary symmetric channel. 
Equation 1.52 is often presented in the form:

$$
\begin{aligned}
P\left(X_{i} / Y_{j}\right) & =\frac{P\left(X_{i}, Y_{j}\right)}{P\left(Y_{j}\right)} \\
& =\frac{P\left(Y_{j}\right) \cdot P\left(X_{i} / Y_{j}\right)}{P\left(Y_{j}\right)}
\end{aligned}
$$

which is referred to as Bayes' rule.

Logically, the probability of receiving a particular $Y_{j}=Y_{j_{0}}$ is the sum of all joint probabilities $P\left(X_{i}, Y_{j_{0}}\right)$ over the range of $X_{i}$. This corresponds to the probability of receiving the transmitted $X_{i}$ correctly, giving rise to the channel output $Y_{j_{0}}$ plus the sum of the probabilities of all other possible transmitted symbols giving rise to $Y_{j_{0}}$ :

$$
P\left(Y_{j}\right)=\sum_{X} P\left(X_{i}, Y_{j}\right)=\sum_{X} P\left(X_{i}\right) P\left(Y_{j} / X_{i}\right)
$$

Similarly:

$$
P\left(X_{i}\right)=\sum_{Y} P\left(X_{i}, Y_{j}\right)=\sum_{Y} P\left(Y_{j}\right) P\left(X_{i} / Y_{j}\right)
$$

\subsubsection{Mutual Information}

In this section, we elaborate further on the ramifications of Shannon's information theory [24$27,35,36]$. Over nonideal channels impairments are introduced, and the received information might be different from the transmitted information. In this section, we quantify the amount of information that can be inferred from the received symbols over noisy channels. In the spirit of Shannon's fundamental work [24] and Carlson's classic reference [31], let us continue our discourse with the definition of mutual information. We have already used the notation $P\left(X_{i}\right)$ to denote the probability that the source symbol $X_{i}$ was transmitted and $P\left(Y_{i}\right)$ to denote the probability that the symbol $Y_{j}$ was received. The joint probability that $X_{i}$ was transmitted and $Y_{j}$ was received had been quantified by $P\left(X_{i}, Y_{j}\right)$, and $P\left(X_{i} / Y_{j}\right)$ indicated the conditional probability that $X_{i}$ was transmitted, given that $Y_{j}$ was received, while $P\left(Y_{j} / X_{i}\right)$ was used for the conditional probability that $Y_{j}$ was received given that $X_{i}$ was transmitted.

In case of $i=j$, the conditional probabilities $P\left(Y_{j} / X_{j}\right) j=1 \cdots q$ represent the errorfree transmission probabilities of the source symbols $j=1 \cdots q$. For example, in Figure 1.18 the probabilities $P\left(Y_{0} / X_{0}\right)$ and $P\left(Y_{1} / X_{1}\right)$ are the probabilities of the error-free reception of a transmitted $X_{0}$ and $X_{1}$ source symbol, respectively. The probabilities $P\left(Y_{j} / X_{i}\right) j \neq i$, on the other hand, give the individual error probabilities, which are characteristic of error events that corrupted a transmitted symbol $X_{i}$ to a received symbol of $Y_{j}$. The corresponding error probabilities in Figure 1.18 are $P\left(Y_{0} / X_{1}\right)$ and $P\left(Y_{1} / X_{0}\right)$.

Let us define the mutual information of $X_{i}$ and $Y_{j}$ as:

$$
I\left(X_{i}, Y_{j}\right)=\log _{2} \frac{P\left(X_{i} / Y_{j}\right)}{P\left(X_{i}\right)}=\log _{2} \frac{P\left(X_{i}, Y_{j}\right)}{P\left(X_{i}\right) \cdot P\left(Y_{j}\right)}=\log _{2} \frac{P\left(Y_{j} / X_{i}\right)}{P\left(Y_{j}\right)} \text { bits, }
$$


which quantifies the amount of information conveyed, when $X_{i}$ is transmitted and $Y_{j}$ is received. Over a perfect, noiseless channel, each received symbol $Y_{j}$ uniquely identifies a transmitted symbol $X_{i}$ with a probability of $P\left(X_{i} / Y_{j}\right)=1$. Substituting this probability in Equation 1.56 yields a mutual information of:

$$
I\left(X_{i}, Y_{j}\right)=\log _{2} \frac{1}{P\left(X_{i}\right)}
$$

which is identical to the self-information of $X_{i}$ and hence no information is lost over the channel. If the channel is very noisy and the error probability becomes 0.5 , then the received symbol $Y_{j}$ becomes unrelated to the transmitted symbol $X_{i}$, since for a binary system upon its reception there is a probability of 0.5 that $X_{0}$ was transmitted and the probability of $X_{1}$ is also 0.5 . Then formally $X_{i}$ and $Y_{j}$ are independent and hence

$$
P\left(X_{i} / Y_{j}\right)=\frac{P\left(X_{i}, Y_{j}\right)}{P\left(Y_{j}\right)}=\frac{P\left(X_{i}\right) \cdot P\left(Y_{j}\right)}{P\left(Y_{j}\right)}=P\left(X_{i}\right),
$$

giving a mutual information of:

$$
I\left(X_{i}, Y_{j}\right)=\log _{2} \frac{P\left(X_{i}\right)}{P\left(X_{i}\right)}=\log _{2} 1=0
$$

implying that no information is conveyed via the channel. Practical communications channels perform between these extreme values and are usually characterized by the average mutual information defined as:

$$
\begin{aligned}
I(X, Y) & =\sum_{x, y} P\left(X_{i}, Y_{j}\right) \cdot I\left(X_{i}, Y_{j}\right) \\
& =\sum_{x, y} P\left(X_{i}, Y_{j}\right) \cdot \log _{2} \frac{P\left(X_{i} / Y_{j}\right)}{P\left(X_{i}\right)}[\text { bit/symbol } .
\end{aligned}
$$

Clearly, the average mutual information in Equation 1.60 is computed by weighting each component $I\left(X_{i}, Y_{j}\right)$ by its probability of occurrence $P\left(X_{i}, Y_{j}\right)$ and summing these contributions for all combinations of $X_{i}$ and $Y_{j}$. The average mutual information $I(X, Y)$ defined above gives the average amount of source information acquired per received symbol, as distinguished from that per source symbol, which was given by the entropy $H(X)$. Let us now consolidate these definitions by working through the following numerical example.

\subsubsection{Mutual Information Example}

Using the same numeric values as in our introductory example as regards to the binary symmetric channel in Section 1.10.1, and exploiting that from Bayes' rule in Equation 1.53, we have:

$$
P\left(X_{i} / Y_{j}\right)=\frac{P\left(X_{i}, Y_{j}\right)}{P\left(Y_{j}\right)}
$$


The following probabilities can be derived, which will be used at a later stage, in order to determine the mutual information:

$$
P\left(X_{1} / Y_{1}\right)=P(1 / 1)=\frac{P(1,1)}{P_{1}}=\frac{0.686}{0.692} \approx 0.9913
$$

and

$$
P\left(X_{0} / Y_{0}\right)=P(0 / 0)=\frac{P(0,0)}{P_{0}}=\frac{0.294}{0.3080} \approx 0.9545,
$$

where $P_{1}=0.692$ and $P_{0}=0.3080$ represent the total probability of receiving 1 and 0 , respectively, which is the union of the respective events of error-free and erroneous receptions yielding the specific logical value concerned. The mutual information from Equation 1.56 is computed as:

$$
\begin{aligned}
I\left(X_{1}, Y_{1}\right) & =\log _{2} \frac{P\left(X_{1} / Y_{1}\right)}{P\left(X_{1}\right)} \\
& \approx \log _{2} \frac{0.9913}{0.7} \approx 0.502 \mathrm{bit} \\
I\left(X_{0}, Y_{0}\right) & \approx \log _{2} \frac{0.9545}{0.3} \approx 1.67 \mathrm{bit} .
\end{aligned}
$$

These figures must be contrasted with the amount of source information conveyed by the source symbols $X_{0}, X_{1}$ :

$$
I(0)=\log _{2} \frac{1}{0.3} \approx \log _{2} 3.33 \approx 1.737 \mathrm{bit} / \mathrm{symbol}
$$

and

$$
I(1)=\log _{2} \frac{1}{0.7} \approx \log _{2} 1.43 \approx 0.5146 \mathrm{bit} / \mathrm{symbol} .
$$

The amount of information "lost" in the noisy channel is given by the difference between the amount of information carried by the source symbols and the mutual information gained upon inferring a particular symbol at the noisy channel's output. Hence, the lost information can be computed from Equations $1.61,1.62,1.63$, and 1.64, yielding $(1.737-1.67) \approx 0.067$ bit and $(0.5146-0.502) \approx 0.013$ bit, respectively. These values may not seem catastrophic, but in relative terms they are quite substantial and their values rapidly escalate, as the channel error probability is increased. For the sake of completeness and for future use, let us compute the remaining mutual information terms, namely, $I\left(X_{0}, Y_{1}\right)$ and $I\left(X_{1}, Y_{0}\right)$, which necessitate 
the computation of:

$$
\begin{aligned}
P\left(X_{0} / Y_{1}\right) & =\frac{P\left(X_{0}, Y_{1}\right)}{P\left(Y_{1}\right)} \\
P(0 / 1) & =\frac{P(0,1)}{P_{1}}=\frac{0.3 \cdot 0.02}{0.692} \approx 0.00867 \\
P\left(X_{1} / Y_{0}\right) & =\frac{P\left(X_{1}, Y_{0}\right)}{P\left(Y_{0}\right)} \\
P(1 / 0) & =\frac{P(1,0)}{P_{0}}=\frac{0.7 \cdot 0.02}{0.308} \approx 0.04545 \\
I\left(X_{0}, Y_{1}\right) & =\log _{2} \frac{P\left(X_{0} / Y_{1}\right)}{P\left(X_{0}\right)} \approx \log _{2} \frac{0.00867}{0.3} \approx-5.11 \mathrm{bit} \\
I\left(X_{1}, Y_{0}\right) & =\log _{2} \frac{P\left(X_{1} / Y_{0}\right)}{P\left(X_{1}\right)} \approx \log _{2} \frac{0.04545}{0.7} \approx-3.945 \mathrm{bit},
\end{aligned}
$$

where the negative sign reflects the amount of "misinformation" as regards, for example, $X_{0}$ upon receiving $Y_{1}$. In this example we informally introduced the definition of mutual information. Let us now set out to formally exploit the benefits of our deeper insight into the effects of the noisy channel.

\subsubsection{Information Loss via Imperfect Channels}

Upon rewriting the definition of mutual information in Equation 1.56, we have:

$$
\begin{aligned}
I\left(X_{i}, Y_{j}\right) & =\log _{2} \frac{P\left(X_{i} / Y_{j}\right)}{P\left(X_{i}\right)} \\
& =\log _{2} \frac{1}{P\left(X_{i}\right)}-\log _{2} \frac{1}{P\left(X_{i} / Y_{j}\right)} \\
& =I\left(X_{i}\right)-I\left(X_{i} / Y_{j}\right) .
\end{aligned}
$$

Following Shannon's [24-27, 35, 36] and Ferenczy's [33] approach and rearranging Equation 1.67 yields:

$$
\underbrace{I\left(X_{i}\right)}_{\text {Source Inf. Inf. conveyed to rec. }}-\underbrace{I\left(X_{i}, Y_{j}\right)}_{\text {Inf. loss }}=
$$

Briefly returning to figure 1.18 assists the interpretation of $P\left(X_{i} / Y_{j}\right)$ as the probability or certainty/uncertainty that $X_{i}$ was transmitted, given that $Y_{j}$ was received, which justifies the above definition of the information loss. It is useful to observe from this figure that, as it was stated before, $P\left(Y_{j} / X_{i}\right)$ represents the probability of erroneous or error-free reception. Explicitly, if $j=i$, then $P\left(Y_{j} / X_{i}\right)=P\left(Y_{j} / X_{j}\right)$ is the probability of error-free reception, while if $j \neq i$, then $P\left(Y_{j} / X_{i}\right)$ is the probability of erroneous reception. 
With the probability $P\left(Y_{j} / X_{i}\right)$ of erroneous reception in mind, we can actually associate an error information term with it:

$$
I\left(Y_{j} / X_{i}\right)=\log _{2} \frac{1}{P\left(Y_{j} / X_{i}\right)}
$$

Let us now concentrate on the average mutual information's expression in Equation 1.60 and expand it as follows:

$$
\begin{aligned}
I(X, Y) & =\sum_{X, Y} P\left(X_{i}, Y_{j}\right) \cdot \log _{2} \frac{1}{P\left(X_{i}\right)} \\
& -\sum_{X, Y} P\left(X_{i}, Y_{j}\right) \log _{2} \frac{1}{P\left(X_{i} / Y_{j}\right)} .
\end{aligned}
$$

Considering the first term at the right-hand side (rhs) of the above equation and invoking Equation 1.55, we have:

$$
\sum_{X}\left[\sum_{Y} P\left(X_{i}, Y_{j}\right)\right] \log _{2} \frac{1}{P\left(X_{i}\right)}=\sum_{X} P\left(X_{i}\right) \log _{2} \frac{1}{P\left(X_{i}\right)}=H(X) .
$$

Then rearranging Equation 1.70 gives:

$$
H(X)-I(X, Y)=\sum_{X, Y} P\left(X_{i}, Y_{j}\right) \log _{2} \frac{1}{P\left(X_{i} / Y_{j}\right)},
$$

where $H(X)$ is the average source information per symbol and $I(X, Y)$ is the average conveyed information per received symbol.

Consequently, the rhs term must be the average information per symbol lost in the noisy channel. As we have seen in Equation 1.67 and Equation 1.68, the information loss is given by:

$$
I\left(X_{i} / Y_{j}\right)=\log _{2} \frac{1}{P\left(X_{i} / Y_{j}\right)}
$$

The average information loss $H(X / Y)$ equivocation, which Shannon [26] terms is computed as the weighted sum of these components:

$$
H(X / Y)=\sum_{X} \sum_{Y} P\left(X_{i}, Y_{j}\right) \cdot \log _{2} \frac{1}{P\left(X_{i} / Y_{j}\right)} .
$$

Following Shannon, this definition allowed us to express Equation 1.72 as:

$$
\underbrace{H(X)}_{\text {(av. source inf/sym.) }}-\underbrace{I(X, Y)}_{\text {(av. conveyed inf/sym.) }}=\underbrace{H(X / Y)}_{\text {(av. lost inf/sym.) }}
$$




\subsubsection{Error Entropy via Imperfect Channels}

Similarly to our previous approach and using the probability $P\left(Y_{j} / X_{i}\right)$ of erroneous reception associated with the information term of:

$$
I\left(Y_{j} / X_{i}\right)=\log _{2} \frac{1}{P\left(Y_{j} / X_{i}\right)}
$$

we can define the average "error information" or error entropy. Hence, the above error information terms in Equation 1.76 are weighted using the probabilities $P\left(X_{i}, Y_{j}\right)$ and averaged for all $X$ and $Y$ values, defining the error entropy:

$$
H(Y / X)=\sum_{X} \sum_{Y} P\left(X_{i}, Y_{j}\right) \log _{2} \frac{1}{P\left(Y_{j} / X_{i}\right)} .
$$

Using Bayes' rule from Equation 1.52, we have

$$
\begin{aligned}
P\left(X_{i} / Y_{j}\right) \cdot P\left(Y_{j}\right) & =P\left(Y_{j} / X_{i}\right) \cdot P\left(X_{i}\right) \\
\frac{P\left(X_{i} / Y_{j}\right)}{P\left(X_{i}\right)} & =\frac{P\left(Y_{j} / X_{i}\right)}{P\left(Y_{j}\right)} .
\end{aligned}
$$

Following from this, for the average mutual information in Equation 1.56 we have:

$$
I(X, Y)=I(Y, X)
$$

which, after interchanging $X$ and $Y$ in Equation 1.75, gives:

$$
\underbrace{H(Y)}_{\text {nation entropy }}-\underbrace{I(Y, X)}_{\text {conveyed inf }}=\underbrace{H(Y / X)}_{\text {error entropy }} .
$$

Subtracting the conveyed information from the destination entropy gives the error entropy, which is nonzero, if the destination entropy and conveyed information are not equal due to channel errors. Let us now proceed following Ferenczy's approach [33] and summarize the most important definitions for future reference in Table 1.7 before we attempt to augment their physical interpretations using the forthcoming numerical example.

Example Using the BSC model of Figure 1.16, as an extension of the worked examples of Subsections 1.10.1 and 1.10.4 and following Ferenczy's interpretation [33] of Shannon's elaborations [24-27,35,36], let us compute the following range of system characteristics:

(a) The joint information, as distinct from the mutual information introduced earlier, for all possible channel input/output combinations.

(b) The entropy, i.e., the average information of both the source and the sink.

(c) The average joint information $H(X, Y)$.

(d) The average mutual information per symbol conveyed.

(e) The average information loss and average error entropy. 


\begin{tabular}{|l|l|}
\hline Quantity & Definition \\
\hline Source inf. & $I\left(X_{i}\right)=-\log _{2} P\left(X_{i}\right)$ \\
\hline Received inf. & $I\left(Y_{j}\right)=-\log _{2} P\left(Y_{j}\right)$ \\
\hline Joint inf. & $I_{X_{i}, Y_{j}}=-\log _{2} P\left(X_{i}, Y_{j}\right)$ \\
\hline Mutual inf. & $I\left(X_{i}, Y_{j}\right)=\log _{2} \frac{P\left(X_{i} / Y_{j}\right)}{P\left(X_{i}\right)}$ \\
\hline Av. Mut. inf. & $I(X, Y)=\sum_{X} \sum_{Y} P\left(X_{i} Y_{j}\right) \log _{2} \frac{P\left(X_{i} / Y_{j}\right)}{P\left(X_{i}\right)}$ \\
\hline Source entropy & $H(X)=-\sum_{X} P\left(X_{i}\right) \cdot \log _{2} P\left(X_{i}\right)$ \\
\hline Destination entr. & $H(Y)=-\sum_{Y} P\left(Y_{j}\right) \log _{2} P\left(Y_{j}\right)$ \\
\hline Equivocation & $H(X / Y)=-\sum_{X} \sum_{Y} P\left(X_{i}, Y_{j}\right) \log _{2} P\left(X_{i} / Y_{j}\right)$ \\
\hline Error entropy & $H(Y / X)=-\sum_{X} \sum_{Y} P\left(X_{i} Y_{j}\right) \log _{2} P\left(Y_{j} / X_{i}\right)$ \\
\hline
\end{tabular}

Table 1.7: Summary of Definitions (C)Ferenczy [33]

With reference to Figure 1.16 and to our introductory example from Section 1.10.1 we commence by computing further parameters of the BSC. Recall that the source information was:

$$
\begin{aligned}
& I\left(X_{0}\right)=\log _{2} \frac{1}{0.3} \approx 3.322 \log _{10} 3.333 \approx 1.737 \text { bit } \\
& I\left(X_{1}\right)=\log _{2} \frac{1}{0.7} \approx 0.515 \text { bit. }
\end{aligned}
$$

The probability of receiving a logical 0 was 0.308 and that of logical 1 was 0.692 , of whether 0 or 1 was transmitted. Hence, the information inferred upon the reception of 0 and 1 , respectively, is given by:

$$
\begin{aligned}
& I\left(Y_{0}\right)=\log _{2} \frac{1}{0.308} \approx 3.322 \log _{10} 3.247 \approx 1.699 \text { bit } \\
& I\left(Y_{1}\right)=\log _{2} \frac{1}{0.692} \approx 0.531 \text { bit. }
\end{aligned}
$$

Observe that because of the reduced probability of receiving a logical 1 from $0.7 \rightarrow$ 0.692 as a consequence of channel-induced corruption, the probability of receiving a logical 0 is increased from $0.3 \rightarrow 0.308$. This is expected to increase the average destination entropy, since the entropy maximum of unity is achieved, when the symbols are equiprobable. We note, however, that this does not give more information about the source symbols, which must be maximized in an efficient communications system. In our example, the information conveyed increases for the reduced probability logical 1 from 0.515 bit $\rightarrow 0.531$ bit and decreases for the increased probability 0 from 1.737 bit $\rightarrow 1.699$ bit. Furthermore, the average information conveyed is reduced, since the reduction from 1.737 to 1.699 bit is more than the increment from 0.515 to 0.531 . In the extreme case of an error probability of 0.5 we would have $P(0)=P(1)=0.5$, and $I(1)=I(0)=1$ bit, associated with receiving equiprobable random bits, which again would have a maximal destination entropy, but a minimal information concerning the source symbols transmitted. Following the above interesting 
introductory calculations, let us now turn our attention to the computation of the joint information.

a/ The joint information, as distinct from the mutual information introduced earlier in Equation 1.56, of all possible channel input/output combinations is computed from Figure 1.16 as follows:

$$
\begin{aligned}
I_{X_{i}, Y_{j}} & =-\log _{2} P\left(X_{i}, Y_{j}\right) \\
I_{00} & =-\log _{2}(0.3 \cdot 0.98) \approx-3.322 \cdot \log _{10} 0.294 \approx 1.766 \mathrm{bit} \\
I_{01} & =-\log _{2}(0.3 \cdot 0.02) \approx 7.381 \mathrm{bit} \\
I_{10} & =-\log _{2}(0.7 \cdot 0.02) \approx 6.159 \mathrm{bit} \\
I_{11} & =-\log _{2}(0.7 \cdot 0.98) \approx 0.544 \mathrm{bit} .
\end{aligned}
$$

These information terms can be individually interpreted formally as the information carried by the simultaneous occurrence of the given symbol combinations. For example, as it accrues from their computation, $I_{00}$ and $I_{11}$ correspond to the favorable event of error-free reception of a transmitted 0 and 1, respectively, which hence were simply computed by formally evaluating the information terms. By the same token, in the computation of $I_{01}$ and $I_{10}$, the corresponding source probabilities were weighted by the channel error probability rather than the error-free transmission probability, leading to the corresponding information terms. The latter terms, namely, $I_{01}$ and $I_{10}$, represent low-probability, high-information events due to the low channel error probability of 0.02 .

Lastly, a perfect channel with zero error probability would render the probability of the error-events zero, which in turn would assign infinite information contents to the corresponding terms of $I_{01}$ and $I_{10}$, while $I_{00}$ and $I_{11}$ would be identical to the selfinformation of the 0 and 1 symbols. Then, if under zero error probability we evaluate the effect of the individual symbol probabilities on the remaining joint information terms, the less frequently a symbol is emitted by the source, the higher its associated joint information term becomes and vice versa, which is seen by comparing $I_{00}$ and $I_{11}$. Their difference can be equalized by assuming an identical probability of 0.5 for both, which would yield $I_{00}=I_{11}=1$-bit. The unweighted average of $I_{00}$ and $I_{11}$ would then be lower than in case of the previously used probabilities of 0.3 and 0.7 , respectively, since the maximum average would be associated with the case of 0 and 1 , where the associated $\log _{2}$ terms would be 0 and $-\infty$, respectively. The appropriately weighted average joint information terms will be evaluted under paragraph c/ during our later calculations. Let us now move on to evaluate the average information of the source and sink. 
b/ Calculating the entropy, that is, the average information for both the source and the sink, is quite straightforward and ensues as follows:

$$
\begin{aligned}
H(X) & =\sum_{i=1}^{2} P\left(X_{i}\right) \cdot \log _{2} \frac{1}{P\left(X_{i}\right)} \\
& \approx 0.3 \cdot \log _{2} 3.333+0.7 \cdot \log _{2} 1.429 \\
& \approx 0.5211+0.3605 \\
& \approx 0.8816 \mathrm{bit} / \mathrm{symbol} .
\end{aligned}
$$

For the computation of the sink's entropy, we invoke Equations 1.49 and 1.50, yielding:

$$
\begin{aligned}
H(Y) & =0.308 \cdot \log _{2} \frac{1}{0.308}+0.692 \log _{2} \frac{1}{0.692} \\
& \approx 0.5233+0.3676 \\
& \approx 0.8909 \mathrm{bit} / \text { symbol. }
\end{aligned}
$$

Again, the destination entropy $H(Y)$ is higher than the source entropy $H(X)$ due to the more random reception caused by channel errors, approaching $H(Y)=1 \mathrm{bit} / \mathrm{symbol}$ for a channel bit error rate of 0.5. Note, however, that unfortunately this increased destination entropy does not convey more information about the source itself.

c/ Computing the average joint information $H(X, Y)$ gives:

$$
\begin{aligned}
H(X, Y) & =-\sum_{i=1}^{2} \sum_{j=1}^{2} P\left(X_{i}, Y_{j}\right) \log _{2} P\left(X_{i}, Y_{j}\right) \\
& =-\sum_{i=1}^{2} \sum_{j=1}^{2} P\left(X_{i}, Y_{j}\right) I_{X_{i}, Y_{j}} .
\end{aligned}
$$

Upon substituting the $I_{X_{i}, Y_{j}}$ values calculated in Equation 1.81 into Equation 1.84, we have:

$$
\begin{aligned}
H(X, Y) \approx & 0.3 \cdot 0.98 \cdot 1.766+0.3 \cdot 0.02 \cdot 7.381 \\
& +0.7 \cdot 0.02 \cdot 6.159+0.7 \cdot 0.98 \cdot 0.544 \\
\approx & 0.519+0.044+0.086+0.373 \\
\approx & 1.022 \mathrm{bit} / \text { symbol-combination } .
\end{aligned}
$$

In order to interpret $H(X, Y)$, let us again scrutinize the definition given in Equation 1.84, which weights the joint information terms of Equation 1.81 by their probability of occurence. We have argued before that the joint information terms corresponding to erroneous events are high due to the low error probability of 0.02 . Observe, therefore, that these high-information symbol combinations are weighted by their low-probability of occurrence, causing $H(X, Y)$ to become relatively low. It is also instructive to consider the above terms in Equation 1.84 for the extreme cases of 
zero and 0.5 error probabilities and for different source emission probabilities, which are left for the reader to explore. Here we proceed considering the average conveyed mutual information per symbol.

d/ The average conveyed mutual information per symbol was defined in Equation 1.60 in order to quantify the average source information acquired per received symbol, which is repeated here for convenience as follows:

$$
\begin{aligned}
I(X, Y) & =\sum_{X} \sum_{Y} P\left(X_{i}, Y_{j}\right) \log _{2} \frac{P\left(X_{i} / Y_{j}\right)}{P\left(X_{i}\right)} \\
& =\sum_{X} \sum_{Y} P\left(X_{i}, Y_{j}\right) \cdot I\left(X_{i}, Y_{j}\right) .
\end{aligned}
$$

Using the individual mutual information terms from Equations 1.61-1.66 in Section 1.10.4, we get the average mutual information representing the average amount of source information acquired from the received symbols, as follows:

$$
\begin{aligned}
I(X, Y) \approx & 0.3 \cdot 0.98 \cdot 1.67+0.3 \cdot 0.02 \cdot(-5.11) \\
& +0.7 \cdot 0.02 \cdot(-3.945)+0.7 \cdot 0.98 \cdot 0.502 \\
\approx & 0.491-0.03066-0.05523+0.3444 \\
\approx & 0.7495 \mathrm{bit} / \mathrm{symbol} .
\end{aligned}
$$

In order to interpret the concept of mutual information, in Section 1.10.4 we noted that the amount of information "lost" owing to channel errors was given by the difference between the amount of information carried by the source symbols and the mutual information gained upon inferring a particular symbol at the noisy channel's output. These were given in Equations $1.61-1.64$, yielding $(1.737-1.67) \approx 0.067$ bit and $(0.5146-0.502) \approx 0.013$ bit, for the transmission of a 0 and 1 , respectively. We also noted that the negative sign of the terms corresponding to the error-events reflected the amount of misinformation as regards, for example, $X_{0}$ upon receiving $Y_{1}$. Over a perfect channel, the cross-coupling transitions of Figure 1.16 are eliminated, since the associated error probabilities are 0 , and hence there is no information loss over the channel. Consequently, the error-free mutual information terms become identical to the self-information of the source symbols, since exactly the same amount of information can be inferred upon reception of a symbol, as much is carried by its appearance at the output of the source.

It is also instructive to study the effect of different error probabilities and source symbol probabilities in the average mutual information definition of Equation 1.84 in order to acquire a better understanding of its physical interpretation and quantitative power as regards the system's performance. It is interesting to note, for example, that assuming an error probability of zero will therefore result in average mutual information, which is identical to the source and destination entropy computed above under paragraph $\mathbf{b} /$. It is also plausible that $I(X, Y)$ will be higher than the previously computed 0.7495 bits/symbol, if the symbol probabilities are closer to 0.5 , or in general in case of $q$-ary 
sources closer to $1 / q$. As expected, for a binary symbol probability of 0.5 and error probability of 0 , we have $I(X, Y)=1$ bit/symbol.

e/ Lastly, let us determine the average information loss and average error entropy, which were defined in Equations 1.74 and 1.80 and are repeated here for convenience. Again, we will be using some of the previously computed probabilities from Sections 1.10 .1 and 1.10 .4 , beginning with computation of the average information loss of Equation 1.74:

$$
\begin{aligned}
H(X / Y)= & -\sum_{X} \sum_{Y} P\left(X_{i}, Y_{j}\right) \log _{2} P\left(X_{i} / Y_{j}\right) \\
= & -P\left(X_{0}, Y_{0}\right) \log _{2} P\left(X_{0} / Y_{0}\right)-P\left(X_{0}, Y_{1}\right) \log _{2} P\left(X_{0} / Y_{1}\right) \\
& -P\left(X_{1}, Y_{0}\right) \log _{2} P\left(X_{1} / Y_{0}\right)-P\left(X_{1}, Y_{1}\right) \log _{2} P\left(X_{1} / Y_{1}\right) \\
= & P(0,0) \cdot \log _{2} P(0 / 0)+P(0,1) \cdot \log _{2} P(0 / 1) \\
& P(1,0) \cdot \log _{2} P(1 / 0)+P(1,1) \cdot \log _{2} P(1 / 1) \\
\approx & -0.3 \cdot 0.98 \cdot \log _{2} 0.9545-0.3 \cdot 0.02 \cdot \log _{2} 0.00867 \\
& -0.7 \cdot 0.02 \cdot \log _{2} 0.04545-0.7 \cdot 0.98 \cdot \log _{2} 0.9913 \\
\approx & 0.0198+0.0411+0.0624+0.0086 \\
\approx & 0.132 \mathrm{bit} / \mathrm{symbol} .
\end{aligned}
$$

In order to augment the physical interpretation of the above-average information loss expression, let us examine the main contributing factors in it. It is expected to decrease as the error probability decreases. Although it is not straightforward to infer the clear effect of any individual parameter in the equation, experience shows that as the error probability increases, the two middle terms corresponding to the error events become more dominant. Again, the reader may find it instructive to alter some of the parameters on a one-by-one basis and study the way its influence manifests itself in terms of the overall information loss. 
Moving on to the computation of the average error entropy, we find its definition equation is repeated below, and on inspecting Figure 1.16 we have:

$$
\begin{aligned}
H(Y / X)= & -\sum_{X} \sum_{Y} P\left(X_{i}, Y_{j}\right) \cdot \log _{2} P\left(Y_{j} / X_{i}\right) \\
= & -P\left(X_{0}, Y_{0}\right) \log _{2} P\left(Y_{0} / X_{0}\right)-P\left(X_{0}, Y_{1}\right) \log _{2} P\left(Y_{1} / X_{0}\right) \\
& -P\left(X_{1}, Y_{0}\right) \log _{2} P\left(Y_{0} / X_{1}\right)-P\left(X_{1}, Y_{1}\right) \log _{2} P\left(Y_{1} / X_{1}\right) \\
P\left(Y_{0} / X_{0}\right)= & 0.98 \\
P\left(Y_{0} / X_{1}\right)= & 0.02 \\
P\left(Y_{1} / X_{0}\right)= & 0.02 \\
P\left(Y_{1} / X_{1}\right)= & 0.98 \\
H(Y / X)= & P(0,0) \cdot \log _{2} P(0 / 0)+P(0,1) \cdot \log _{2} P(0 / 1) \\
& P(1,0) \cdot \log _{2} P(1 / 0)+P(1,1) \cdot \log _{2} P(1 / 1) \\
= & -0.294 \cdot \log _{2} 0.98-0.014 \cdot \log _{2} 0.02 \\
& -0.006 \cdot \log _{2} 0.02-0.686 \cdot \log _{2} 0.98 \\
\approx & 0.0086+0.079+0.034+0.02 \\
\approx & 0.141 \mathrm{bit} / \operatorname{symbol}
\end{aligned}
$$

The average error entropy in the above expression is expected to fall as the error probability is reduced and vice versa. Substituting different values into its definition equation further augments its practical interpretation. Using our previous results in this section, we see that the average loss of information per symbol or equivocation denoted by $H(X / Y)$ is given by the difference between the source entropy of Equation 1.82 and the average mutual information of Equation 1.85, yielding:

$$
H(X / Y)=H(X)-I(X, Y) \approx 0.8816-0.7495 \approx 0.132 \mathrm{bit} / \mathrm{symbol},
$$

which according to Equation 1.75, is identical to the value of $H(X / Y)$ computed earlier. In harmony with Equation 1.80, the error entropy can also be computed as the difference of the average entropy $H(Y)$ in Equation 1.83 of the received symbols and the mutual information $I(X, Y)$ of Equation 1.85, yielding:

$$
H(Y)-I(X, Y) \approx 0.8909-0.7495 \approx 0.141 \mathrm{bit} / \mathrm{symbol},
$$

as seen above for $H(Y / X)$.

Having defined the fundamental parameters summarized in Table 1.7 and used in the information-theoretical characterization of communications systems, let us now embark on the definition of channel capacity. Initially, we consider discrete noiseless channels, leading to a brief discussion of noisy discrete channels, and then we proceed to analog channels, before exploring the fundamental message of the Shannon-Hartley law. 


\subsection{Capacity of Discrete Channels [26,33]}

Shannon [26] defined the channel capacity $C$ of a channel as the maximum achievable information transmission rate at which error-free transmission can be maintained over the channel.

Every practical channel is noisy, but transmitting at a sufficiently high power the channel error probability $p_{e}$ can be kept arbitrarily low, providing us with a simple initial channel model for our further elaborations. Following Ferenczy's approach [33], assume that the transmission of symbol $X_{i}$ requires a time interval of $t_{i}$, during which an average of

$$
H(X)=\sum_{i=1}^{q} P\left(X_{i}\right) \log _{2} \frac{1}{P\left(X_{i}\right)} \frac{\text { bit }}{\text { symbol }}
$$

information is transmitted, where $q$ is the size of the source alphabet used. This approach assumes that a variable-length coding algorithm, such as the previously described ShannonFano or the Huffman coding algorithm may be used in order to reduce the transmission rate to as low as the source entropy. Then the average time required for the transmission of a source symbol is computed by weighting $t_{i}$ with the probability of occurrence of symbol $X_{i}, i=1 \ldots q$ :

$$
t_{a v}=\sum_{i=1}^{q} P\left(X_{i}\right) t_{i} \frac{\mathrm{sec}}{\text { symbol }} .
$$

Now we can compute the average information transmission rate $v$ by dividing the average information content of a symbol by the average time required for its transmission:

$$
v=\frac{H(X)}{t_{a v}} \frac{\text { bit }}{\mathrm{sec}}
$$

The maximum transmission rate $v$ as a function of the symbol probability $P\left(X_{i}\right)$ must be found. This is not always an easy task, but a simple case occurs when the symbol duration is constant; that is, we have $t_{i}=t_{0}$ for all symbols. Then the maximum of $v$ is a function of $P\left(X_{i}\right)$ only and we have shown earlier that the entropy $H(X)$ is maximized by equiprobable source symbols, where $P\left(X_{i}\right)=\frac{1}{q}$. Then from Equations 1.86 and 1.87 we have an expression for the channel's maximum capacity:

$$
C=v_{\max }=\frac{H(X)}{t_{a v}}=\frac{\log _{2} q}{t_{0}} \frac{\text { bit }}{\mathrm{sec}} .
$$

Shannon [26] characterized the capacity of discrete noisy channels using the previously defined mutual information describing the amount of average conveyed information, given by:

$$
I(X, Y)=H(Y)-H(Y / X),
$$

where $H(Y)$ is the average amount of information per symbol at the channel's output, while $\mathrm{H}(\mathrm{Y} / \mathrm{X})$ is the error entropy. Here a unity symbol-rate was assumed for the sake of simplicity. Hence, useful information is transmitted only via the channel if $H(Y)>H(Y / X)$. Via a channel with $p_{e}=0.5$, where communication breaks down, we have $H(Y)=H(Y / X)$, and the information conveyed becomes $I(X, Y)=0$. The amount of information conveyed 


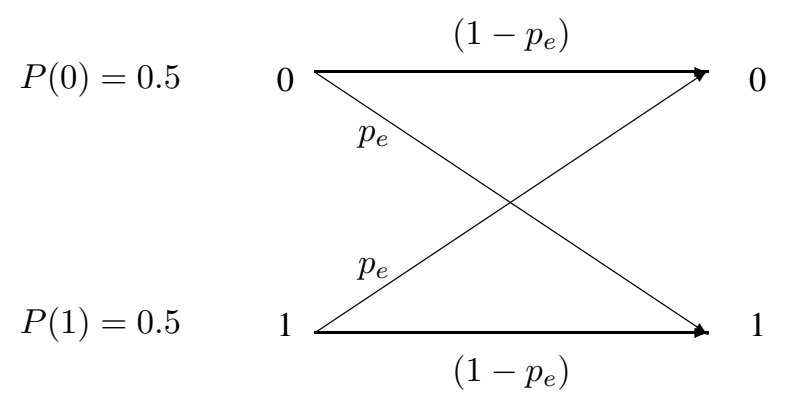

Figure 1.19: BSC model.

is maximum if the error entropy $H(Y / X)=0$. Therefore, Shannon [26] defined the noisy channel's capacity as the maximum value of the conveyed information $I(X, Y)$ :

$$
C=I(X, Y)_{M A X}=[H(Y)-H(Y / X)]_{M A X},
$$

where the maximization of Equation 1.91 is achieved by maximizing the first term and minimizing the second term.

In general, the maximization of Equation 1.91 is an arduous task, but for the BSC seen in Figure 1.19 it becomes fairly simple. Let us consider this simple case and assume that the source probabilities of 1 and 0 are $P(0)=P(1)=0.5$ and the error probability is $p_{e}$. The entropy at the destination is computed as:

$$
H(Y)=-\frac{1}{2} \log _{2} \frac{1}{2}-\frac{1}{2} \log _{2} \frac{1}{2}=1 \mathrm{bit} / \mathrm{symbol},
$$

while the error entropy is given by:

$$
H(Y / X)=-\sum_{X} \sum_{Y} P\left(X_{i}, Y_{j}\right) \cdot \log _{2} P\left(Y_{j} / X_{i}\right)
$$

In order to be able to compute the capacity of the BSC as a function of the channel's error probability, let us substitute the required joint probabilities of:

$$
\begin{aligned}
& P(0,0)=P(0)\left(1-p_{e}\right) \\
& P(0,1)=P(0) p_{e} \\
& P(1,0)=P(1) p_{e} \\
& P(1,1)=P(1)\left(1-p_{e}\right) .
\end{aligned}
$$


and the conditional probabilities of:

$$
\begin{aligned}
& P(0 / 0)=\left(1-p_{e}\right) \\
& P(0 / 1)=p_{e} \\
& P(1 / 0)=p_{e} \\
& P(1 / 1)=\left(1-p_{e}\right) .
\end{aligned}
$$

into Equation 1.92, yielding:

$$
\begin{aligned}
H(Y / X)= & -\left[P(0)\left(1-p_{e}\right) \cdot \log _{2}\left(1-p_{e}\right)+P(0) \cdot p_{e} \log _{2} p_{e}\right. \\
& \left.+P(1) \cdot p_{e} \log _{2} p_{e}+P(1)\left(1-p_{e}\right) \log _{2}\left(1-p_{e}\right)\right] \\
= & -[P(0)+P(1)]\left(1-p_{e}\right) \log _{2}\left(1-p_{e}\right) \\
& +[P(0)+P(1)] p_{e} \log _{2} p_{e} \\
= & -\left(1-p_{e}\right) \cdot \log _{2}\left(1-p_{e}\right)-p_{e} \cdot \log _{2} p_{e} .
\end{aligned}
$$

Finally, upon substituting $H(Y)$ and $H(Y / X)$ from above into Equation 1.91, the BSC's channel capacity becomes:

$$
C=1+\left(1-p_{e}\right) \log _{2}\left(1-p_{e}\right)+p_{e} \log _{2} p_{e}
$$

Following Ferenczy's [33] interpretation of Shannon's lessons [24-27, 35, 36], the graphic representation of the BSC's capacity is depicted in Figure 1.20 using various $p_{e}$ error probabilities.

Observe, for example, that for $p_{e}=10^{-2}$ the channel capacity is $C \approx 0.9 \mathrm{bit} / \mathrm{symbol}$, that is, close to its maximum of $C=1 \mathrm{bit} / \mathrm{symbol}$, but for higher $p_{e}$ values it rapidly decays, falling to $C=0.5 \mathrm{bit} / \mathrm{symbol}$ around $p_{e}=10^{-1}$. If $p_{e}=50 \%$, we have $C=$ 0 bit/symbol; since no useful information transmission takes place, the channel delivers random bits. Notice also that if $P(0) \neq P(1) \neq 0.5$, then $H(Y)<1$ bit/symbol and hence $C<C_{\max }=1 \mathrm{bit} / \mathrm{symbol}$, even if $p_{e}=0$.

\subsection{Shannon's Channel Coding Theorem [30,38]}

In the previous section, we derived a simple expression for the capacity of the noisy BSC in Equation 1.96, which was depicted in Figure 1.20 as a function of the channel's error probability $p_{e}$. In this section, we focus on Shannon's channel coding theorem, which states that as long as the information transmission rate does not exceed the channel's capacity, the bit error rate can be kept arbitrarily low [35,36]. In the context of the BSC channel capacity curve of Figure 1.20, this theorem implies that noise over the channel does not preclude the reliable transmission of information; it only limits the rate at which transmission can take place. Implicitly, this theorem prophesies the existence of an appropriate error correction code, which adds redundancy to the original information symbols. This reduces the system's useful information throughput but simultaneously allows error correction coding. Instead of providing a rigorous proof of this theorem, following the approach suggested by Abramson [30], which was also used by Hey and Allen [38] in their compilation of Feyman's lectures, we will make it plaussible. 


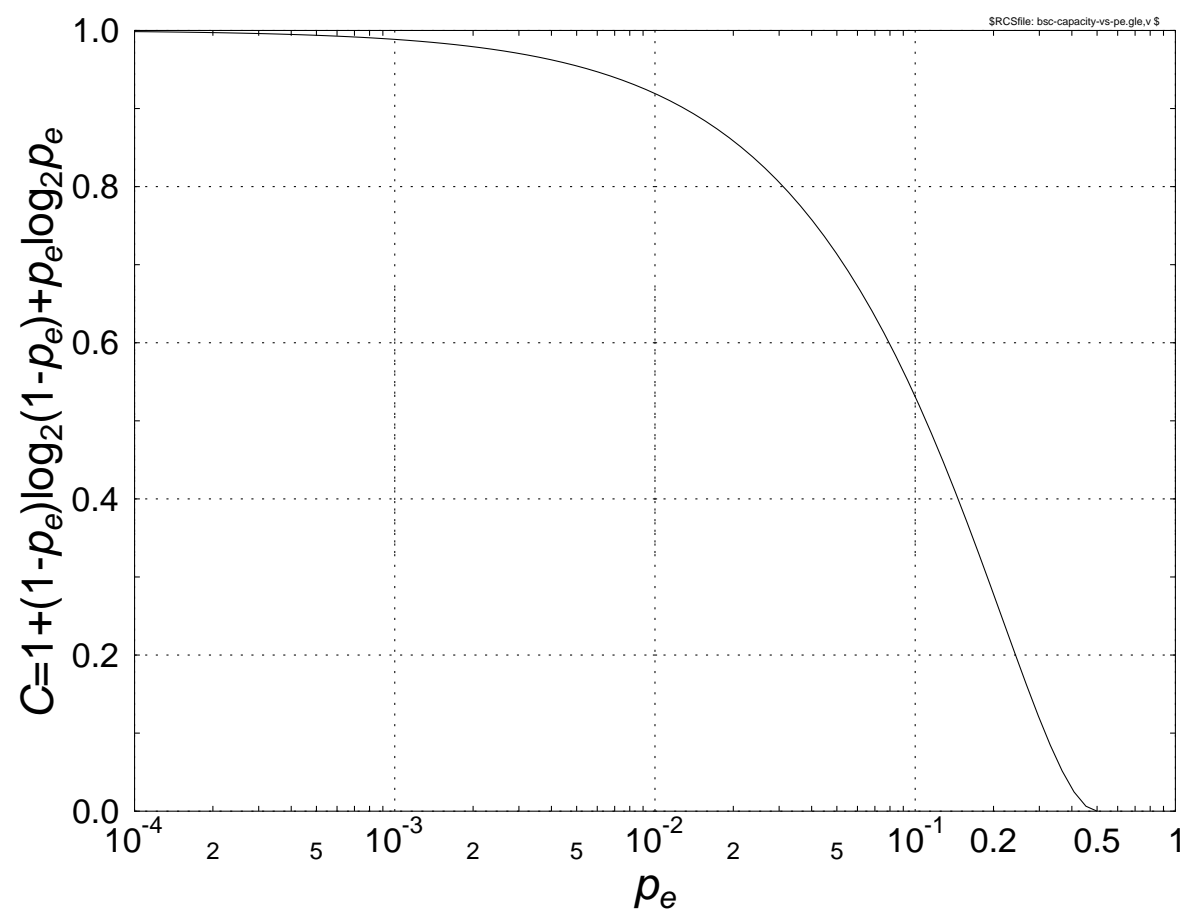

Figure 1.20: Channel capacity versus $p_{e}$ for the BSC.

The theorem is stated more formally as follows. Let us assume that a message of $K$ useful information symbols is transmitted by assigning it to an $N$-symbol so-called block code, where the symbols are binary and the error probability is $p_{e}$. Then, according to Shannon, upon reducing the coding rate $R=\frac{K}{N}$ beyond every limit, the error probability obeys the following relationship:

$$
R=\frac{K}{N} \leq C=1+\left(1-p_{e}\right) \log _{2}\left(1-p_{e}\right)+p_{e} \cdot \log _{2} p_{e} .
$$

As Figure 1.20 shows upon increasing the bit error rate $p_{e}$, the channel capacity reduces gradually toward zero, which forces the channel coding rate $R=\frac{K}{N}$ to zero in the limit. This inequality therefore implies that an arbitrarily low BER is possible only when the coding rate $R$ tends to zero, which assumes an infinite-length block code and an infinite coding delay. By scrutinizing Figure 1.20, we can infer that, for example, for a BER of $10^{-1}$ an approximately $R=\frac{K}{N} \approx \frac{1}{2}$ so-called half-rate code is required in order to achieve asymptotically perfect communications, while for $B E R=10^{-2}$ an approximately $R \approx 0.9$ code is required.

Shannon's channel coding theorem does not specify how to create error correction codes, which can achieve this predicted performance; it merely states their existence. Hence, the 
error correction coding community has endeavored over the years to create such good codes but until 1993 had only limited success. Then in that year Berrou et al. [39] invented the family of iteratively decoded turbo-codes, which are capable of approaching the Shannonian predictions within a fraction of a dB.

Returning to the channel coding theorem, Hey and Feynman [38] offered a witty approach to deepening the physical interpretation of this theorem, which we briefly highlight below. Assuming that the block-coded sequences are long, in each block on the average there are $t=p_{e} \cdot N$ number of errors. In general, $t$ number of errors can be allocated over the block of $N$ positions in

$$
C_{N}^{t}=\left(\begin{array}{c}
N \\
t
\end{array}\right)=\frac{N !}{t !(N-t) !}
$$

different ways, which are associated with the same number of error patterns. The number of additional parity bits added during the coding process is $P=(N-K)$, which must be sufficiently high for identifying all the $C_{N}^{t}$ number of error patterns, in order to allow inverting (i.e., correcting) the corrupted bits in the required positions. Hence, we have [38]:

$$
\frac{N !}{t !(N-t) !} \leq 2^{(N-K)}
$$

Upon exploiting the Stirling formula of

$$
N ! \approx \sqrt{2 \pi N} \cdot\left(\frac{N}{e}\right)^{N}=\sqrt{2 \pi} \cdot \sqrt{N} \cdot N^{N} \cdot e^{-N}
$$

and taking the logarithm of both sides, we have:

$$
\log _{e} N ! \approx \log _{e} \sqrt{2 \pi}+\frac{1}{2} \log _{e} N+N \log _{e} N-N
$$

Furthermore, when $N$ is large, the first and second terms are diminishingly small in comparison to the last two terms. Thus, we have:

$$
\log _{e} N ! \approx N \log _{e} N-N
$$

Then, after taking the logarithm, the factorial expression on the left-hand side (L) of Equation 1.98 can be written as:

$$
L \approx\left[N \log _{e} N-N\right]-\left[t \log _{e} t-t\right]-\left[(N-t) \log _{e}(N-t)-(N-t)\right] .
$$


Now taking into account that $t \approx p_{e} \cdot N$, we have [38]:

$$
\begin{aligned}
L= & {\left[N \log _{e} N-N\right]-\left[p_{e} N \log _{e}\left(p_{e} N\right)-p_{e} N\right] } \\
& -\left[\left(N-p_{e} N\right) \log _{e}\left(N-p_{e} N\right)-\left(N-p_{e} N\right)\right] \\
\approx & {\left[N \log _{e} N-N\right]-\left[p_{e} N \log _{e} p_{e}+p_{e} N \log _{e} N-p_{e} N\right] } \\
& -\left[N \log _{e}\left(N\left(1-p_{e}\right)\right)-p_{e} N \log _{e}\left(N\left(1-p_{e}\right)\right)-\left(N-p_{e} N\right)\right] \\
\approx \quad & {\left[N \log _{e} N-N\right]-\left[p_{e} N \log _{e} p_{e}+p_{e} N \log _{e} N-p_{e} N\right] } \\
& -\left[N \log _{e} N+N \log _{e}\left(1-p_{e}\right)-p_{e} N \log _{e} N\right. \\
& \left.\quad-p_{e} N \log _{e}\left(1-p_{e}\right)-\left(N-p_{e} N\right)\right] \\
\approx \quad & N\left[\log _{e} N-1-p_{e} \log _{e} p_{e}-p_{e} \log _{e} N+p_{e}\right. \\
& \quad-\log _{e} N-\log _{e}\left(1-p_{e}\right)+p_{e} \log _{e} N \\
& \left.\quad+p_{e} \log _{e}\left(1-p_{e}\right)+1-p_{e}\right] \\
\approx & N\left[-p_{e} \log _{e} p_{e}-\log _{e}\left(1-p_{e}\right)+p_{e} \log _{e}\left(1-p_{e}\right)\right] \\
\approx & N\left[-p_{e} \log _{e} p_{e}-\left(1-p_{e}\right) \log _{e}\left(1-p_{e}\right)\right]
\end{aligned}
$$

If we consider that $\log _{e} a=\log _{2} a \cdot \log _{e} 2$, then we can convert the $\log _{e}$ terms to $\log _{2}$ as follows [38]:

$$
L \approx N \log _{e} 2\left[-p_{e} \log _{2} p_{e}-\left(1-p_{e}\right) \log _{2}\left(1-p_{e}\right)\right] .
$$

Finally, upon equating this term with the logarithm of the right-hand side expression of Equation 1.98 , we arrive at:

$$
N \log _{e} 2\left[-p_{e} \log _{2} p_{e}-\left(1-p_{e}\right) \log _{2}\left(1-p_{e}\right)\right] \leq(N-K) \log _{e} 2,
$$

which can be simplified to:

$$
-p_{e} \log _{2} p_{e}-\left(1-p_{e}\right) \log _{2}\left(1-p_{e}\right) \leq 1-\frac{K}{N}
$$

or to a form, identical to Equation 1.97:

$$
\frac{K}{N} \leq 1+\left(1-p_{e}\right) \log _{2}\left(1-p_{e}\right)+p_{e} \log _{2} p_{e} .
$$

\subsection{Capacity of Continuous Channels [27,33]}

During our previous discussions, it was assumed that the source emitted discrete messages with certain finite probabilities, which would be exemplified by an 8-bit analog-to-digital converter emitting one of 256 discrete values with a certain probability. However, after digital source encoding and channel encoding according to the basic schematic of Figure 1.1 the modulator typically converts the digital messages to a finite set of bandlimited analog waveforms, which are chosen for maximum "transmission convenience." In this context, transmission convenience can imply a range of issues, depending on the communications channel. Two typical constraints are predominantly power-limited or bandwidth-limited channels, although in many practical scenarios both of these constraints become important. Because 
of their limited solar power supply, satellite channels tend to be more severely power-limited than bandlimited, while typically the reverse situation is experienced in mobile radio systems.

The third part of Shannon's pioneering paper [27] considers many of these issues. Thus, in what follows we define the measure of information for continuous signals, introduce a concept for the continuous channel capacity, and reveal the relationships among channel bandwidth, channel capacity, and channel signal-to-noise ratio, as stated by the Shannon-Hartley theorem. Finally, the ideal communications system transpiring from Shannon's pioneering work is characterized, before concluding with a brief discussion of the ramifications of wireless channels as regards the applicability of Shannon's results.

Let us now assume that the channel's analog input signal $x(t)$ is bandlimited and hence that it is fully characterized by its Nyquist samples and by its probability density function (PDF) $p(x)$. The analogy of this continuous PDF and that of a discrete source are characterized by $P\left(X_{i}\right) \approx p\left(X_{i}\right) \Delta X$, which reflects the practical way of experimentally determining the histogram of a bandlimited analog signal by observing the relative frequency of events, when its amplitude resides in a $\Delta X$ wide amplitude bin-centered around $X_{i}$. As an analogy to the discrete average information or entropy expression of:

$$
H(X)=-\sum_{i} P\left(X_{i}\right) \cdot \log _{2} P\left(X_{i}\right)
$$

Shannon [27] introduced the entropy of analog sources, as it was also noted and exploited, for example, by Ferenczy [33], as follows:

$$
H(x)=-\int_{-\infty}^{\infty} p(x) \log _{2} p(x) d x .
$$

For our previously used discrete sources, we have shown that the source entropy is maximized for equiprobable messages. The question that arises is whether this is also true for continuous PDFs. Shannon [27] derived the maximum of the analog signal's entropy under the constraints of:

$$
\begin{aligned}
\int_{-\infty}^{\infty} p(x) d x & =1 \\
\sigma_{x}^{2} & =\int_{-\infty}^{\infty} x^{2} \cdot p(x) d x=\text { Constant }
\end{aligned}
$$

based on the calculus of variations. He showed that the entropy of a signal $x(t)$ having a constant variance of $\sigma_{x}^{2}$ is maximum, if $x(t)$ has a Gaussian distribution given by:

$$
p(x)=\frac{1}{\sqrt{2 \pi} \sigma} e^{-\left(x^{2} / 2 \sigma^{2}\right)} .
$$

Then the maximum of the entropy can be derived upon substituting this PDF into the expression of the entropy. Let us first take the natural logarithm of both sides of the PDF and convert it to base two logarithm by taking into account that $\log _{e} a=\log _{2} a \cdot \log _{e} 2$, in order to be able to use it in the entropy's $\log _{2}$ expression. Then the PDF of Equation 1.103 can be 
written as:

$$
-\log _{2} p(x)=+\log _{2} \sqrt{2 \pi} \sigma+\left(x^{2} / 2 \sigma^{2}\right) \cdot \frac{1}{\log _{e} 2},
$$

and upon exploiting that $\log _{e} 2=1 / \log _{2} e$, the entropy is expressed according to Shannon [27] and Ferenczy [33] as:

$$
\begin{aligned}
H_{\text {max }}(x) & =-\int p(x) \cdot \log _{2} p(x) d x \\
& =\int p(x) \cdot \log _{2} \sqrt{2 \pi} \sigma d x+\int p(x) \frac{x^{2} \cdot \log _{2} e}{2 \sigma^{2}} d x \\
& =\log _{2} \sqrt{2 \pi} \sigma \int p(x) d x+\frac{\log _{2} e}{2 \sigma^{2}} \underbrace{\int x^{2} p(x) d x}_{\sigma^{2}} \\
& =\log _{2} \sqrt{2 \pi} \sigma+\frac{\sigma^{2}}{2 \sigma^{2}} \log _{2} e \\
& =\log _{2} \sqrt{2 \pi} \sigma+\frac{\log _{2} e}{2} \\
& =\log _{2} \sqrt{2 \pi} \sigma+\frac{1}{2} \log _{2} e \\
& =\log _{2} \sqrt{2 \pi e} \sigma .
\end{aligned}
$$

Since the maximum of the entropy is proportional to the logarithm of the signal's average power $S_{x}=\sigma_{x}^{2}$, upon quadrupling the signal's power the entropy is increased by one bit because the range of uncertainty as regards where the signal samples can reside is expanded.

We are now ready to formulate the channel capacity versus channel bandwidth and versus channel SNR relationship of analog channels. Let us assume white, additive, signalindependent noise with a power of $N$ via the channel. Then the received (signal+noise) power is given by:

$$
\sigma_{y}^{2}=S+N
$$

By the same argument, the channel's output entropy is maximum if its output signal $y(t)$ has a Gaussian PDF and its value is computed from Equation 1.105 as:

$$
H_{\max }(y)=\frac{1}{2} \log _{2}\left(2 \pi e \sigma_{y}^{2}\right)=\frac{1}{2} \log _{2} 2 \pi e(S+N) .
$$

We proceed by taking into account the channel impairments, reducing the amount of information conveyed by the amount of the error entropy $H(y / x)$ giving:

$$
I(x, y)=H(y)-H(y / x)
$$

where again the noise is assumed to be Gaussian and hence:

$$
H(y / x)=\frac{1}{2} \log _{2}(2 \pi e N)
$$


Upon substituting Equation 1.107 and Equation 1.109 in Equation 1.108, we have:

$$
\begin{aligned}
I(x, y) & =\frac{1}{2} \log _{2}\left(\frac{2 \pi e(S+N)}{2 \pi e N}\right) \\
& =\frac{1}{2} \log _{2}\left(1+\frac{S}{N}\right),
\end{aligned}
$$

where, again, both the channel's output signal and the noise are assumed to have Gaussian distribution.

The analog channel's capacity is then calculated upon multiplying the information conveyed per source sample by the Nyquist sampling rate of $f_{s}=2 \cdot f_{B}$, yielding [35]:

$$
C=f_{B} \cdot \log _{2}\left(1+\frac{S}{N}\right) \frac{\text { bit }}{\mathrm{sec}} .
$$

Equation 1.111 is the well-known Shannon-Hartley law ${ }^{1}$ establishing the relationship among the channel capacity $C$, channel bandwidth $f_{B}$, and channel signal-to-noise ratio (SNR).

Before analyzing the consequences of the Shannon-Hartley law following Shannon's deliberations [35], we make it plausible from a simple practical point of view. As we have seen, the root mean squared (RMS) value of the noise is $\sqrt{N}$, and that of the signal plus noise at the channel's output is $\sqrt{S+N}$. The receiver has to decide from the noisy channel's output signal what signal has been input to the channel, although this has been corrupted by an additive Gaussian noise sample. Over an ideal noiseless channel, the receiver would be able to identify what signal sample was input to the receiver. However, over noisy channels it is of no practical benefit to identify the corrupted received message exactly. It is more beneficial to quantify a discretized version of it using a set of decision threshold values, where the resolution is dependent on how corrupted the samples are. In order to quantify this SNR-dependent receiver dynamic range resolution, let us consider the following argument.

Having very densely spaced receiver detection levels would often yield noise-induced decision errors, while a decision-level spacing of $\sqrt{N}$ according to the RMS noise-amplitude intuitively seems a good compromise between high information resolution and low decision error rate. Then assuming a transmitted sample, which resides at the center of a $\sqrt{N}$ wide decision interval, noise samples larger than $\sqrt{N} / 2$ will carry samples across the adjacent decision boundaries. According to this spacing, the number of receiver reconstruction levels is given by:

$$
q=\frac{\sqrt{S+N}}{\sqrt{N}}=\left(1+\frac{S}{N}\right)^{\frac{1}{2}}
$$

which creates a scenario similar to the transmission of equiprobable $q$-ary discrete symbols via a discrete noisy channel, each conveying $\log _{2} q$ amount of information at the Nyquist sampling rate of $f_{s}=2 \cdot f_{B}$. Therefore, the channel capacity becomes [35]:

$$
C=2 \cdot f_{B} \cdot \log _{2} q=f_{B} \cdot \log _{2}\left(1+\frac{S}{N}\right)
$$

\footnotetext{
${ }^{1}$ Comment by the Authors: Although the loose definition of capacity is due to Hartley, the underlying relationship is entirely due to Shannon.
} 


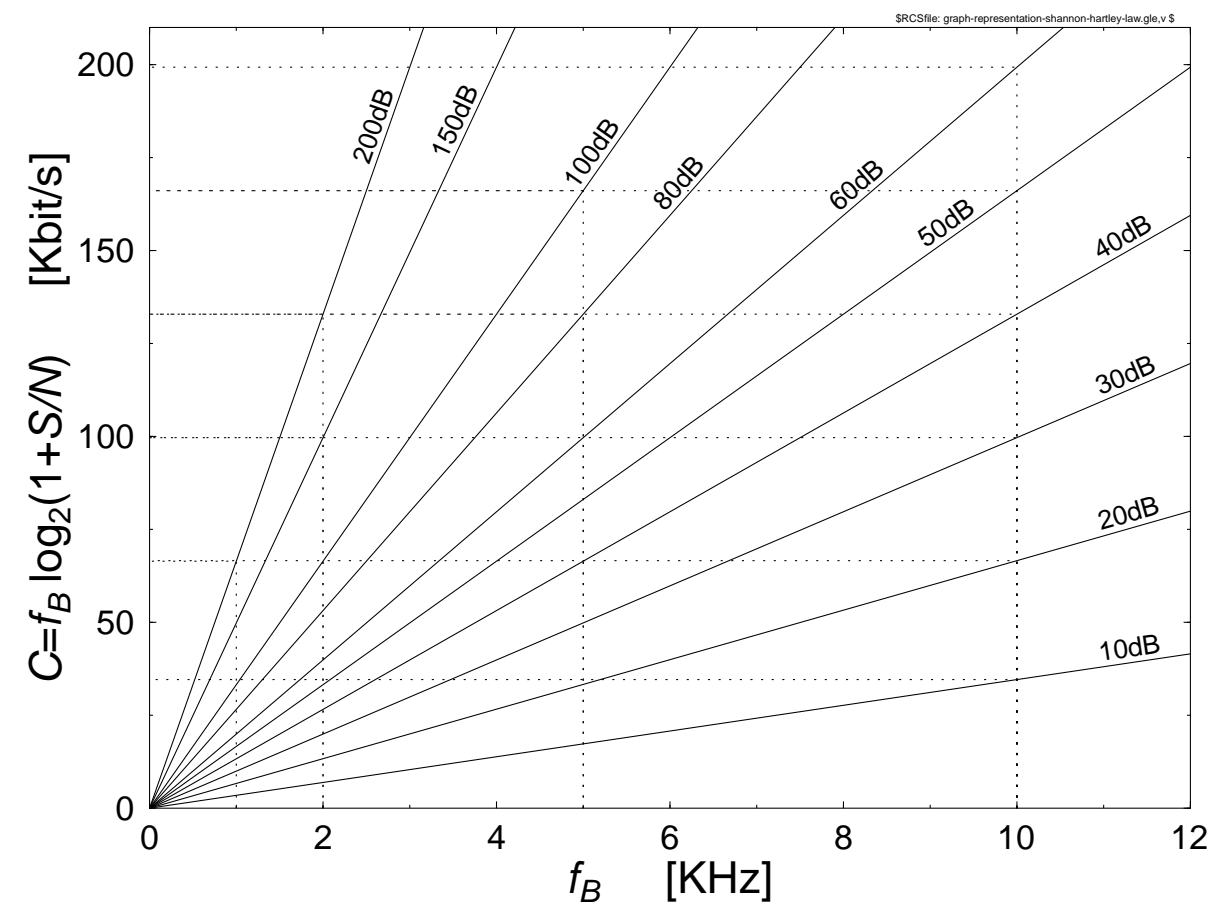

Figure 1.21: Graphical representation of the Shannon-Hartley law. CFerenczy [33].

as seen earlier in Equation 1.111.

\subsubsection{Practical Evaluation of the Shannon-Hartley Law}

The Shannon-Hartley law of Equation 1.111 and Equation 1.113 reveals the fundamental relationship of the SNR, bandwidth, and channel capacity. This relationship can be further studied following Ferenczy's interpretation [33] upon referring to Figure 1.21.

Observe from the figure that a constant channel capacity can be maintained, even when the bandwidth is reduced, if a sufficiently high SNR can be guaranteed. For example, from Figure 1.21 we infer that at $f_{B}=10 \mathrm{KHz}$ and $\mathrm{SNR}=30 \mathrm{~dB}$ the channel capacity is as high as $C=100 \mathrm{kbps}$. Surprisingly, $C \approx 100 \mathrm{kbps}$ can be achieved even for $f_{B}=5 \mathrm{KHz}$, if $\mathrm{SNR}=60 \mathrm{~dB}$ is guaranteed.

Figure 1.22 provides an alternative way of viewing the Shannon-Hartley law, where the SNR is plotted as a function of $f_{B}$, parameterized with the channel capacity $C$. It is important to notice how dramatically the SNR must be increased in order to maintain a constant channel capacity $C$, as the bandwidth $f_{B}$ is reduced below $0.1 \cdot C$, where $C$ and $f_{B}$ are expressed in kbit/s and Hz, respectively. This is due to the $\log _{2}(1+\mathrm{SNR})$ function in Equation 1.111, where a logarithmically increasing SNR value is necessitated to compensate for the linear reduction in terms of $f_{B}$. 


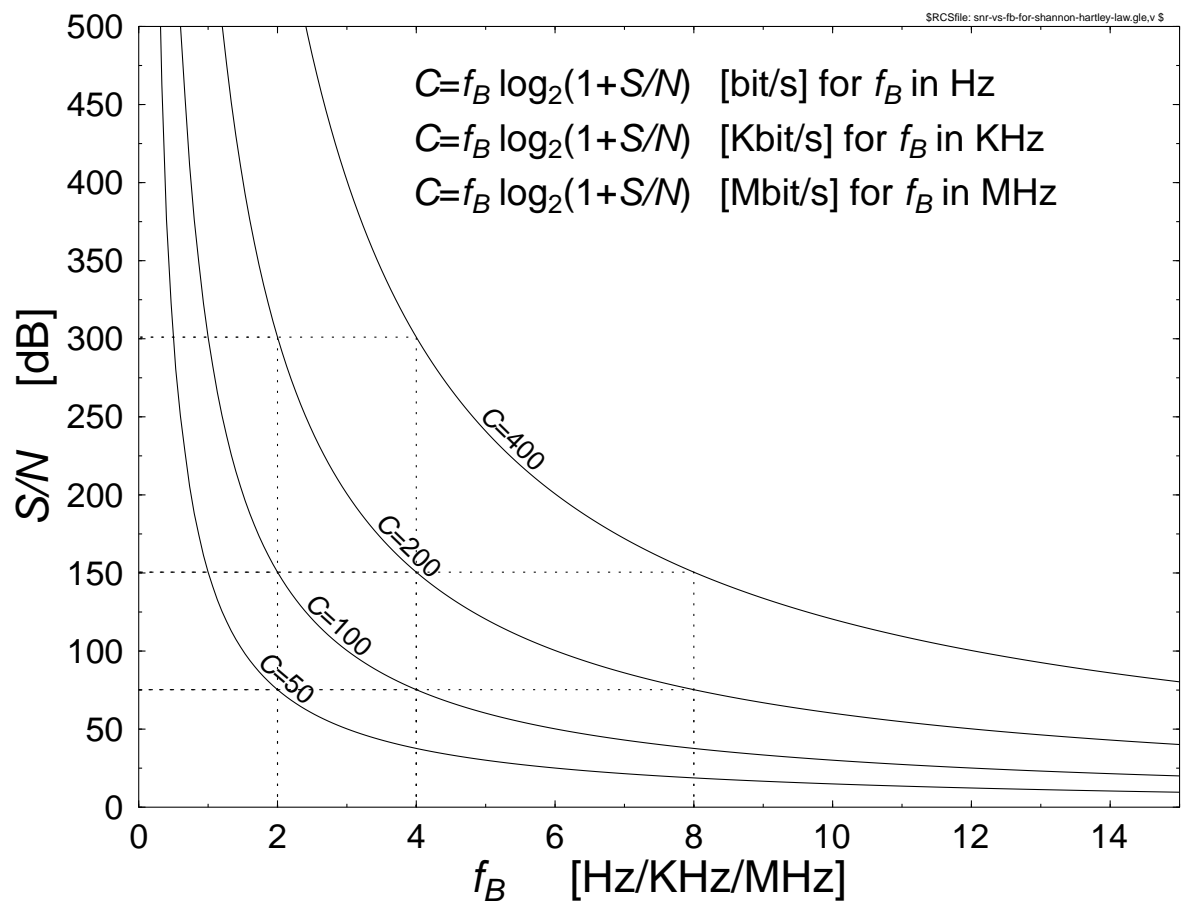

Figure 1.22: SNR versus $f_{B}$ relations according to the Shannon-Hartley law. CFerenczy [33].

From our previous discourse, the relationship between the relative channel capacity $C / f_{B}$ expressed from Equation 1.113, and the channel SNR now becomes plausible. This relationship is quantified in Table 1.8 and Figure 1.23 for convenience. Notice that due to the logarithmic SNR scale expressed in $\mathrm{dBs}$, the $C / f_{B}\left[\frac{b p s}{H z}\right]$ curve becomes near-linear, allowing a near-linearly proportional relative channel capacity improvement upon increasing the channel SNR. A very important consequence of this relationship is that if the channel SNR is sufficiently high to support communications using a high number of modulation levels, the channel is not exploited to its full capacity upon using $C / f_{B}$ values lower than is afforded by the prevailing SNR. Proposing various techniques in order to exploit this philosophy was the motivation of reference [40].

The capacity $C$ of a noiseless channel with $S N R=\infty$ is $C=\infty$, although noiseless channels do not exist. In contrast, the capacity of an ideal system with $f_{B}=\infty$ is finite [31, 34]. Assuming additive white Gaussian noise (AWGN) with a double-sided power spectral density (PSD) of $\eta / 2$, we have $N=\frac{\eta}{2} \cdot 2 \cdot f_{B}=\eta \cdot f_{B}$, and applying the Shannon-Hartley 


\begin{tabular}{|c|c|c|}
\hline \multicolumn{2}{|c|}{ SNR } & $\begin{array}{c}C / f_{B} \\
\mathrm{bit} / \mathrm{sec} / \mathrm{Hz}\end{array}$ \\
\cline { 1 - 2 } Ratio & $\mathrm{dB}$ & 1 \\
1 & 0 & 2 \\
3 & 4.8 & 2 \\
7 & 8.5 & 3 \\
15 & 11.8 & 4 \\
31 & 14.9 & 5 \\
63 & 18.0 & 6 \\
127 & 21.0 & 7 \\
\hline
\end{tabular}

Table 1.8: Relative Channel Capacity versus SNR

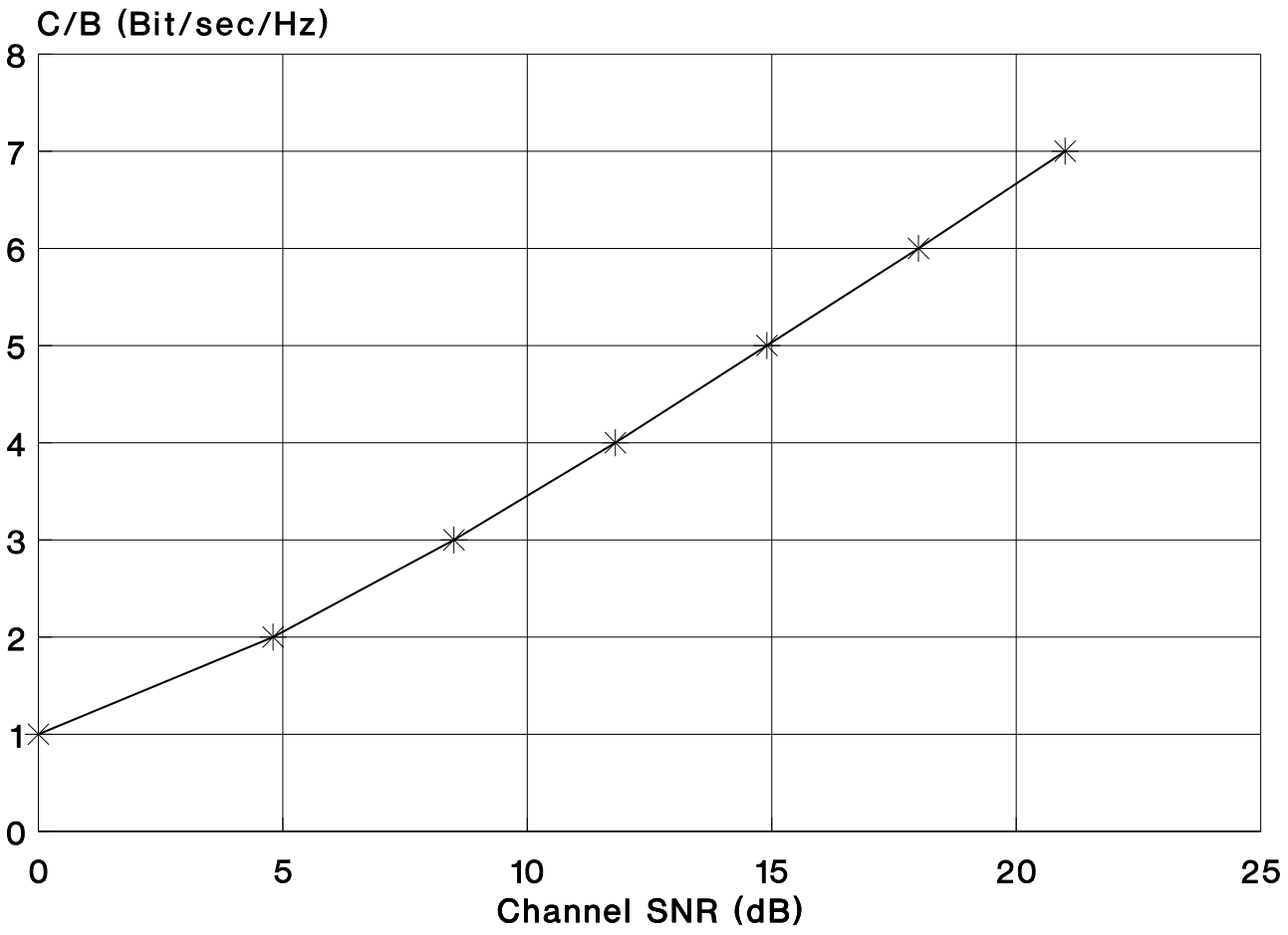

Figure 1.23: Relative channel capacity $\left(C / f_{B}\right)$ versus SNR (dB). 
law gives [31]:

$$
\begin{aligned}
C & =f_{B} \cdot \log _{2}\left(1+\frac{S}{\eta f_{B}}\right) \\
& =\left(\frac{S}{\eta}\right)\left(\frac{\eta f_{B}}{S}\right) \log _{2}\left(1+\frac{S}{\eta f_{B}}\right) \\
& =\left(\frac{S}{\eta}\right) \log _{2}\left(1+\frac{S}{\eta f_{B}}\right)^{\frac{\eta f_{B}}{S}} .
\end{aligned}
$$

Our aim is now to determine $C_{\infty}=\lim _{f_{B} \rightarrow \infty} C$. Upon exploiting that:

$$
\lim _{x \rightarrow 0}(1+x)^{\frac{1}{x}}=e
$$

where $x=S /\left(\eta \cdot f_{B}\right)$, we have

$$
C_{\infty}=\lim _{f_{B} \rightarrow \infty} C=\frac{S}{\eta} \log _{2} e=1.45 \cdot\left(\frac{S}{\eta}\right),
$$

which is the capacity of the channel with $f_{B}=\infty$. The practically achievable transmission rate $R$ is typically less than the channel capacity $C$, although complex turbo-coded modems [39] can approach its value. For example, for a telephone channel with a signal-tonoise ratio of $S / N=10^{3}=30 \mathrm{~d} B$ and a bandwidth of $B=3.4 \mathrm{kHz}$ from Equation 1.113, we have $C=3.4 \cdot \log _{2}\left(1+10^{3}\right) \frac{\mathrm{kbit}}{\mathrm{sec}} \approx 3.4 \cdot 10=34 \mathrm{kbit} / \mathrm{s}$, which is fairly close to the rate of the V.34 CCITT standard $28.8 \mathrm{kbit} / \mathrm{s}$ telephone-channel modem that was recently standardized.

In this chapter, we have been concerned with various individual aspects of Shannon's information theory [24-27,35,36]. Drawing nearer to concluding our discourse on the foundations of information theory, let us now outline in broad terms the main ramifications of Shannon's work [24-27].

\subsubsection{Shannon's Ideal Communications System for Gaussian Channels}

The ideal Shannonian communications system shown in Figure 1.24 has the following characteristics. The system's information-carrying capacity is given by the information rate $C=f_{B} \log _{2}(1+S / N)$, while as regards its error rate we have $p_{e} \rightarrow 0$. The transmitted and received signals are bandlimited Gaussian random variables, which facilitate communicating at the highest possible rate over the channel.

Information from the source is observed for $T$ seconds, where $T$ is the symbol duration and encoded as equiprobable $M$-ary symbols with a rate of $R=\frac{\log _{2} M}{T}$. Accordingly, the signaling waveform generator of Figure 1.24 assigns a bandlimited AWGN representation having a maximum frequency of $f_{B}$ from the set of $M=2^{R T}$ possible waveforms to the source message, uniquely representing the signal $x(t)$ to be transmitted for a duration of $T$. The noisy received signal $y(t)=x(t)+n(t)$ is compared to all $M=2^{R T}$ prestored waveforms at the receiver, and the most "similar" is chosen to identify the most likely transmitted source message. The observation intervals at both the encoder and decoder amount to $T$, 


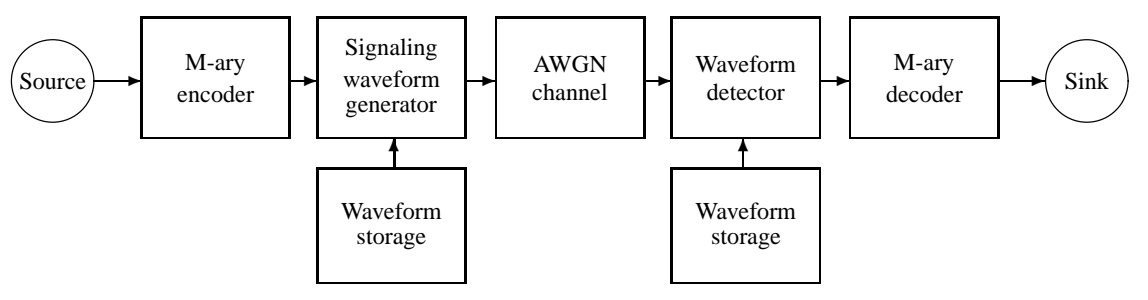

Figure 1.24: Shannon's ideal communications system for AWGN channels.

yielding an overall coding delay of $2 T$. Signaling at a rate equal to the channel capacity is only possible, if the source signal's observation interval is infinitely long, that is, $T \rightarrow \infty$.

Before concluding this chapter, we offer a brief discussion of the system-architectural ramifications of transmitting over wireless channels rather than over AWGN channels.

\subsection{Shannon's Message for Wireless Channels}

In wireless communications over power- and bandlimited channels it is always of prime concern to maintain an optimum compromise in terms of the contradictory requirements of low bit rate, high robustness against channel errors, low delay, and low complexity. The minimum bit rate at which distortionless communications is possible is determined by the entropy of the speech source message. Note, however, that in practical terms the source rate corresponding to the entropy is only asymptotically achievable as the encoding memory length or delay tends to infinity. Any further compression is associated with information loss or coding distortion. Note that the optimum source encoder generates a perfectly uncorrelated sourcecoded stream, where all the source redundancy has been removed; therefore, the encoded symbols are independent, and each one has the same significance. Having the same significance implies that the corruption of any of the source-encoded symbols results in identical source signal distortion over imperfect channels.

Under these conditions, according to Shannon's pioneering work [24], which was expanded, for example, by Hagenauer [41] and Viterbi [42], the best protection against transmission errors is achieved if source and channel coding are treated as separate entities. When using a block code of length $N$ channel coded symbols in order to encode $K$ source symbols with a coding rate of $R=K / N$, the symbol error rate can be rendered arbitrarily low, if $N$ tends to infinity and the coding rate to zero. This condition also implies an infinite coding delay. Based on the above considerations and on the assumption of additive white Gaussian noise (AWGN) channels, source and channel coding have historically been separately optimized.

Mobile radio channels are subjected to multipath propagation and so constitute a more hostile transmission medium than AWGN channels, typically exhibiting path-loss, log-normal slow fading and Rayleigh fast-fading. Furthermore, if the signaling rate used is higher than the channel's coherence bandwidth, over which no spectral-domain linear distortion is experienced, then additional impairments are inflicted by dispersion, which is associated with 
frequency-domain linear distortions. Under these circumstances the channel's error distribution versus time becomes bursty, and an infinite-memory symbol interleaver is required in Figure 1.1 in order to disperse the bursty errors and hence to render the error distribution random Gaussian-like, such as over AWGN channels. For mobile channels, many of the above mentioned, asymptotically valid ramifications of Shannon's theorems have limited applicability.

A range of practical limitations must be observed when designing mobile radio speech or video links. Although it is often possible to further reduce the prevailing typical bit rate of state-of-art speech or video codecs, in practical terms this is possible only after a concomitant increase of the implementational complexity and encoding delay. A good example of these limitations is the half-rate GSM speech codec, which was required to approximately halve the encoding rate of the $13 \mathrm{kbps}$ full-rate codec, while maintaining less than quadrupled complexity, similar robustness against channel errors, and less than doubled encoding delay. Naturally, the increased algorithmic complexity is typically associated with higher power consumption, while the reduced number of bits used to represent a certain speech segment intuitively implies that each bit will have an increased relative significance. Accordingly, their corruption may inflict increasingly objectionable speech degradations, unless special attention is devoted to this problem.

In a somewhat simplistic approach, one could argue that because of the reduced source rate we could accommodate an increased number of parity symbols using a more powerful, implementationally more complex and lower rate channel codec, while maintaining the same transmission bandwidth. However, the complexity, quality, and robustness trade-off of such a scheme may not always be attractive.

A more intelligent approach is required to design better speech or video transceivers for mobile radio channels [41]. Such an intelligent transceiver is portrayed in Figure 1.1. Perfect source encoders operating close to the information-theoretical limits of Shannon's predictions can only be designed for stationary source signals, a condition not satisfied by most source signals. Further previously mentioned limitations are the encoding complexity and delay. As a consequence of these limitations the source-coded stream will inherently contain residual redundancy, and the correlated source symbols will exhibit unequal error sensitivity, requiring unequal error protection. Following Hagenauer [41], we will refer to the additional knowledge as regards the different importance or vulnerability of various speech-coded bits as source significance information (SSI). Furthermore, Hagenauer termed the confidence associated with the channel decoder's decisions as decoder reliability information (DRI). These additional links between the source and channel codecs are also indicated in Figure 1.1. A variety of such techniques have successfully been used in robust source-matched source and channel coding.

The role of the interleaver and de-interleaver seen in Figure 1.1 is to rearrange the channel coded bits before transmission. The mobile radio channel typically inflicts bursts of errors during deep channel fades, which often overload the channel decoder's error correction capability in certain speech or video segments. In contrast other segments are not benefiting from the channel codec at all, because they may have been transmitted between fades and hence are error-free even without channel coding. This problem can be circumvented by dispersing the bursts of errors more randomly between fades so that the channel codec is always faced with an "average-quality" channel, rather than the bimodal faded/nonfaded condition. In other 
words, channel codecs are most efficient if the channel errors are near-uniformly dispersed over consecutive received segments.

In its simplest manifestation, an interleaver is a memory matrix filled with channel coded symbols on a row-by-row basis, which are then passed on to the modulator on a column-bycolumn basis. If the transmitted sequence is corrupted by a burst of errors, the de-interleaver maps the received symbols back to their original positions, thereby dispersing the bursty channel errors. An infinite memory channel interleaver is required in order to perfectly randomize the bursty errors and therefore to transform the Rayleigh-fading channel's error statistics to that of a AWGN channel, for which Shannon's information theoretical predictions apply. Since in interactive video or speech communications the tolerable delay is strictly limited, the interleaver's memory length and efficiency are also limited.

A specific deficiency of these rectangular interleavers is that in case of a constant vehicular speed the Rayleigh-fading mobile channel typically produces periodic fades and error bursts at traveled distances of $\lambda / 2$, where $\lambda$ is the carrier's wavelength, which may be mapped by the rectangular interleaver to another set of periodic bursts of errors. Hence a range of random interleaving algorithms have been proposed in the literature.

Returning to Figure 1.1, the soft-decision information (SDI) or channel state information (CSI) link provides a measure of confidence with regard to the likelihood that a specific symbol was transmitted. Then the channel decoder often uses this information in order to invoke maximum likelihood sequence estimation (MLSE) based on the Viterbi algorithm and thereby improve the system's performance with respect to conventional hard-decision decoding. Following this rudimentary review of Shannon's information theory, let us now turn our attention to the characterization of wireless communications channels.

\subsection{Summary and Conclusions}

An overview of Shannonian information theory has been given, in order to establish a firm basis for our further discussions throughout the book. Initially we focussed our attention on the basic Shannonian information transmission scheme and highlighted the differences between Shannon's theory valid for ideal source and channel codecs as well as for Gaussian channels and its ramifications for Rayleigh channels. We also argued that practical finitedelay source codecs cannot operate at transmission rates as low as the entropy of the source. However, these codecs do not have to operate losslessly, since perceptually unobjectionable distortions can be tolerated. This allows us to reduce the associated bit rate.

Since wireless channels exhibit bursty error statistics, the error bursts can only be randomized with the aid of infinite-length channel interleavers, which are not amenable to real-time communications. Although with the advent of high-delay turbo channel codecs it is possible to operate near the Shannonian performance limits over Gaussian channels, over bursty and dispersive channels different information-theoretical channel capacity limits apply.

We considered the entropy of information sources both with and without memory and highlighted a number of algorithms, such as the Shannon-Fano, the Huffman and run-length coding algorithms, designed for the efficient encoding of sources exhibiting memory. This was followed by considering the transmission of information over noise-contaminated channels leading to Shannon's channel coding theorem. Our discussions continued by considering the capacity of communications channels in the context of the Shannon-Hartley law. The 
chapter was concluded by considering the ramifications of Shannon's messages for wireless channels.

\subsection{Structure and novel aspects of the book}

In this section we provide an overview of the remainder of this book and summarise its novel aspects.

In Chapter 1 we provide a rudimentary introduction to information theory, in order to lay the foundations for the rest of the book, while in Chapter 2, we provide a brief overview of the system components and techniques used throughout the monograph.

In Chapter 6 we demonstrate the application of IrVLCs for the joint source and channel coding of video information, as described in Section 4.1.2. The proposed scheme employs the serial concatenation and iterative decoding of a video codec with a channel codec, in the manner detailed in Section 4.3.3.2. Our novel video codec represents the video information using Variable Dimension Vector Quantisation (VDVQ) tiles, which are similar to the VQ tiles described in Section 4.2.1, but having various dimensions. The VDVQ tiles employed are represented using the corresponding RVLC codewords selected from the VDVQ/RVLC codebook, as described in Section 4.2.5. However, the legitimate use of the VDVQ tiles and their corresponding RVLC codewords is limited by a number of code constraints, which ensure that the VDVQ tiles employed perfectly tessellate, among other desirable design objectives. As a result, different sub-sets of the RVLC codewords are available at different points during the encoding of the video information and the proposed approach adopts an IrVLC philosophy.

In the video codec of Chapter 6, the VDVQ/RVLC-induced code constraints are uniquely and unambiguously described by a novel VDVQ/RVLC trellis structure, which resembles the symbol-based VLEC trellis [1,2] described in Section 4.2.6.3. Hence, the employment of the VDVQ/RVLC trellis structure allows the consideration of all legitimate transmission frame permutations. This fact is exploited in the video encoder in order to perform novel MMSE VDVQ/RVLC encoding, using a variant of the Viterbi algorithm [3] described in Section 4.2.6.2.

Additionally, the employment of the VDVQ/RVLC trellis structure during video decoding guarantees the recovery of legitimate - although not necessarily error-free - video information. As a result, the video decoder never has to discard video information. This is unlike in conventional video decoders, where a single transmission error may render an entire transmission frame invalid. Furthermore, the novel modification of the BCJR algorithm [4] of Section 4.3.2.2 is employed during APP SISO VDVQ/RVLC decoding in order to facilitate the iterative exchange of soft information with the serially concatenated channel decoder and in order to perform the soft MMSE reconstruction of the video sequence. Finally, since the VDVQ/RVLC trellis structure describes the complete set of VDVQ/RVLC-induced code constraints, all of the associated redundancy is beneficially exploited with the aid of the modified BCJR algorithm.

Owing to its aforementioned benefits and its employment of a joint source and channel coding philosophy, the video transmission scheme of Chapter 6 is shown to outperform the corresponding benchmarkers employing a separate source and channel coding philosophy. Our findings were originally published in $[5,6]$. 
In Chapter 7, we investigate the application of IrVLCs to UEP, as described in Section 4.1.3. Here, a number of component VLC codebooks having different error correction capabilities are employed to encode various fractions of the source symbol frame. In the case where the various fractions of the source symbol frame have different error sensitivities, this approach may be expected to yield a higher reconstruction quality than equal protection, as noted in [7-9], for example.

Chapter 7 also investigates the application of IrVLCs to near-capacity operation, as described in Section 4.1.1. Here, a number of component VLC codebooks having different inverted Extrinsic Information Transfer Chart (EXIT) functions are employed to encode various fractions of the source symbol frame. We show that the inverted IrVLC EXIT function may be obtained as a weighted average of the inverted component VLC EXIT functions, as described in Section 4.4. Additionally, the EXIT chart matching algorithm [10] described in Section 4.4 is employed to shape the inverted IrVLC EXIT function to match the EXIT function of a serially concatenated inner channel code and to create a narrow but still open EXIT chart tunnel. In this way, iterative decoding convergence to an infinitesimally low probability of error is facilitated at near capacity SNRs, as described in Section 4.3.4.4.

Furthermore, in Chapter 7, the UEP and near-capacity operation of the described scheme is assessed using novel plots that characterise the computational complexity of iterative decoding. More specifically, the average number of ACS operations required to reconstruct each source symbol with a high quality is plotted against the channel SNR. These plots are employed to compare the novel IrVLC-based scheme with a suitably designed IrCC and regular VLC based benchmarkers, quantifying the advantages of the IrVLCs Furthermore, these plots demonstrate that the complexity associated with the bit-based VLEC trellis of Section 4.2.6.1 is significantly lower than that of the symbol-based trellis described in Section 4.2.6.3. Our findings were originally published in $[11,12]$ and we proposed attractive near-capacity IrVLC schemes in [13-18].

In Chapter 8 we introduce a novel RV-FDM as an alternative to the IV-FD lower bound of (4.8) for the characterisation of the error correction capability that is associated with VLEC codebooks. Unlike the IV-FD lower bound, the RV-FDM assumes values from the real-valued domain, hence allowing the comparison of the error correction capability of two VLEC codebooks having equal IV-FD lower bounds, as described in Section 4.2.6.4. Furthermore, we show that a VLEC codebook's RV-FDM affects the number of inflection points that appear in the corresponding inverted EXIT function. This complements the property [19] that the area below an inverted VLEC EXIT function equals the corresponding coding rate, as well as the property that a free distance of at least two yields an inverted VLEC EXIT function that reaches the top right hand corner of the EXIT chart, as described in Section 4.3.4.4.

These properties are exploited by a novel GA in order to design beneficial VLEC codebooks having arbitrary inverted EXIT function shapes. This is in contrast to the methods of [20-22], which are incapable of designing codebooks having specific EXIT function shapes without imposing a significant level of 'trial-and-error' based human interaction, as described in Section 4.4. This novel GA is shown to be attractive for the design of IrVLC component codebooks for EXIT chart matching, since Chapter 8 also demonstrates that our ability to create open EXIT chart tunnels at near-capacity channel SNRs depends on the availability of a suite of component codes having a wide variety of EXIT function shapes. 
Finally, a suite of component VLEC codebooks designed by the novel GA is found to facilitate higher-accuracy EXIT chart matching than a benchmarker suite designed using the state-of-the-art method of [22]. Our novel RV-FDM and GA were originally published in $[15,16]$.

In Chapter 9, we propose a novel modification to the EXIT chart matching algorithm of [10] that additionally seeks a reduced APP SISO decoding complexity by considering the complexities associated with each of the component codes. Furthermore, another novel modification of Chapter 9 facilitates the EXIT chart matching of irregular codes that employ a suite of component codes having the same coding rate. This is achieved by removing the EXIT chart matching constraint of (4.30), facilitating the design of a novel IrURC.

Additionally, Chapter 9 demonstrates the joint EXIT chart matching of two serially concatenated irregular codecs, namely an outer IrVLC and an inner IrURC. This is achieved by iteratively matching the inverted outer EXIT function to the inner EXIT function and vice versa. By employing an irregular inner code, in addition to an irregular outer code, we can afford a higher degree of design freedom than the proposals of [10], which employ a regular inner code. Hence, the proposed approach is shown to facilitate even nearer-capacity operation, which is comparable to that of IrLDPC and irregular turbo codes, as described in Section 4.4. Our findings were originally published in $[17,18]$ and we additionally demonstated the joint EXIT chart matching of serially concatenated irregular codecs in [23].

Finally, in Chapter 10, we compare the results and findings of the previous chapters and draw our conclusions.

In summary, the novel aspects of this research monograph are:

- a novel VDVQ/RVLC-TCM scheme for the iterative joint source and channel decoding of video information;

- its VDVQ/RVLC trellis structure;

- the adaptation of the Viterbi algorithm for MMSE VDVQ/RVLC encoding;

- the adaptation of the BCJR algorithm for APP SISO VDVQ/RVLC decoding and MMSE video reconstruction;

- IrVLC schemes for near-capacity operation;

- complexity versus channel SNR plots which are parameterised by the reconstruction quality;

- the RV-FDM for characterising the error correction capability of VLECs having the same IV-FD;

- the characterisation of the relationship between a VLEC's RV-FDM and the shape of its inverted EXIT function;

- a GA for designing VLECs having specific EXIT functions;

- a suite of VLECs that are suitable for a wide range of IrVLC applications;

- the adaptation of the EXIT chart matching algorithm to facilitate the use of component codes having the same coding rate;

- the adaptation of the EXIT chart matching algorithm to additionally seek a reduced APP SISO decoding computational complexity;

- the joint EXIT chart matching algorithm for designing schemes employing a serial concatenation of two irregular codecs;

- an IrVLC-IrURC scheme for very near capacity joint source and channel coding. 


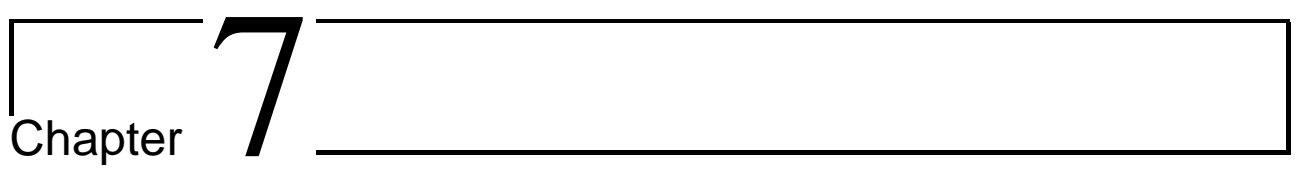

\section{Irregular Variable Length Codes for EXIT Chart Matching}

\subsection{Introduction}

As demonstrated in Section 6.6, a serially concatenated [131] transmission scheme is capable of achieving iterative decoding [132] convergence to an infinitesimally low probability of error at near-capacity Signal to Noise Ratios (SNRs), if the EXtrinsic Information Transfer (EXIT) functions of the inner and outer codecs are well matched. This motivated the design of Irregular Convolutional Coding (IrCC) schemes in [10], as described in Section ??.

The inverted EXIT function of an outer IrCC channel codec can be specifically shaped in order to match the EXIT function of a serially concatenated inner codec. This is possible, because IrCCs amalgamate a number of component Convolutional Codes (CC) [51] having different coding rates, each of which is employed to generate a specific fraction of the IrCCencoded bit stream. As described in Section ??, the composite inverted IrCC EXIT function is given as a weighted average of the inverted EXIT functions of the individual component $\mathrm{CCs}$, where each weight is given by the particular fraction of the IrCC-encoded bit stream that is generated by the corresponding component CC. Hence, it is the specific selection of these fractions that facilitates the shaping of the inverted composite IrCC EXIT function. Using the EXIT chart matching algorithm of [10], the inverted IrCC EXIT chart may be matched to the EXIT function of the inner codec in this way. This facilitates the creation of an open EXIT chart tunnel [158] at low channel SNRs, which approach the channel's capacity bound.

However, the constituent bit-based CCs [51] of the IrCC codec of [10] are unable to exploit the unequal source symbol occurrence probabilities that are typically associated with audio, speech, image and video sources [61,62]. Note that unequal source symbol occurrence probabilities were exemplified in Section 6.3.2. Since the exploitation of all available redundancy is required for near-capacity operation [24], the Huffman source encoder [65] of Chapter 1 must be employed to remove this source redundancy before IrCC encoding commences. However, the reconstruction of the Huffman encoded bits with a particularly low Bit Error Ratio (BER) is required in order that Huffman decoding [65] can achieve a low 
Symbol Error Ratio (SER), owing to its high error sensitivity, which often leads to loss of synchronisation.

This motivates the application of the Variable Length Error Correction (VLEC) code [89] and Reversible Variable Length Coding (RVLC) [99] classes of Variable Length Codes (VLCs) as an alternative to the concatenated Huffman and CC coding of sequences of source symbols having values with unequal probabilities of occurrence. Unlike CCs, these joint source and channel coding VLC schemes are capable of exploiting unequal source symbol occurrence probabilities, as described in Chapter ??. More specifically, source symbols having indices of $k \in[1 \ldots K]$ and associated with unequal probabilities of occurrence $\{P(k)\}_{k=1}^{K}$ are mapped to binary codewords of varying lengths $\left\{I^{k}\right\}_{k=1}^{K}$ from a $K$-entry codebook VLC during VLC encoding. Typically, the more frequently a particular source symbol value occurs, the shorter its VLC codeword, resulting in a reduced average codeword length of

$$
L(\mathbf{V L C})=\sum_{k=1}^{K} P(k) \cdot I^{k} .
$$

In order that each valid VLC codeword sequence may be uniquely decoded, a lower bound equal to the source entropy of

$$
E=-\sum_{k=1}^{K} P(k) \cdot \log _{2}(P(k))
$$

is imposed upon the average codeword length $L(\mathbf{V L C})$. Any discrepancy between $L(\mathbf{V L C})$ and $E$ is quantified by the coding rate of

$$
R(\mathbf{V L C})=\frac{E}{L(\mathbf{V L C})}
$$

and may be attributed to the intentional introduction of redundancy into the VLEC or RVLC codewords. Naturally, this intentionally introduced redundancy imposes code constraints that limit the set of legitimate sequences of VLC-encoded bits. Like the code constraints of CCs [51], the VLC code constraints may be exploited for providing an error correcting capability during VLC decoding [89]. Note that the lower the VLC coding rate, the higher the associated potential error correction capability, as described in Chapter ??. Furthermore, unlike in CC decoding, any redundancy owing to the unequal occurrence probabilities of the source symbol values may also be exploited during VLC decoding [89].

Depending on the coding rate $R$ (VLC) of the VLECs or RVLCs, the associated code constraints render their decoding substantially less sensitive to bit errors than Huffman decoding is, as described in Chapter ??. Hence, a coding gain of $1 \mathrm{~dB}$ at an SER of $10^{-5}$ has been observed by employing VLEC coding having a particular coding rate instead of a concatenated Huffman and Bose-Chaudhuri-Hocquenghem (BCH) $[198,199]$ coding scheme having the same coding rate [89].

Hence the application of EXIT chart matching invoking Irregular Variable Length Coding (IrVLC) is motivated for the sake of near-capacity joint source and channel coding of source symbol sequences having values exhibiting unequal occurrence probabilities. In this 
chapter, we therefore employ a novel IrVLC scheme as our outer source codec, which we serially concatenate $[131,132]$ with an inner channel codec for the sake of exchanging extrinsic information. As shown in Figure 7.1, instead of the component CCs employed in IrCC schemes, the proposed IrVLC scheme employs component VLC codebooks. These have different coding rates and are used for encoding appropriately selected fractions of the input source symbol stream. In this way, the resultant composite inverted EXIT function may be shaped for ensuring that it does not cross the EXIT function of the inner channel codec.

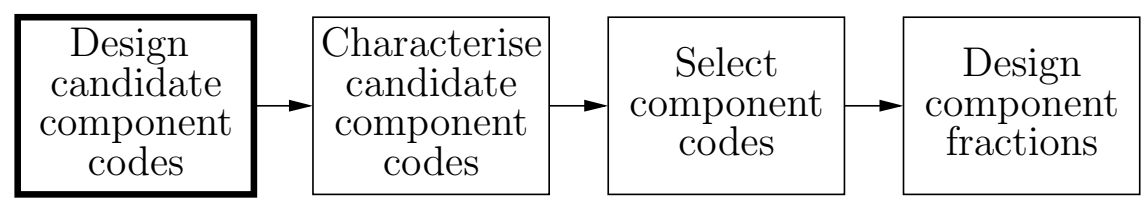

Figure 7.1: Conventional irregular coding design process. This chapter presents modifications to the aspects of this process that are indicated using a bold box.

Note that the proposed scheme has an Unequal Error Protection (UEP) capability [200], since different fractions of the input source symbol stream are protected by different VLC codebooks having different coding rates and, hence, different error correction capabilities. In a manner similar to that of [7-9] for example, this UEP capability may be employed to appropriately protect audio-, speech-, image- and video-encoded bit sequences, which are typically generated using diverse encoding techniques and exhibit various error sensitivities. For example, video coding typically achieves compression by employing Motion Compensation (MC) [64] to exploit the characteristic inter-frame redundancy of video information and the Discrete Cosine Transform (DCT) [63] to exploit the intra-frame redundancy, as described in Section 6.1. As noted in [61], typically a higher degree of video reconstruction distortion typically results from transmission errors that affect the MC-generated motion vectors than from those inflicted on the DCT-encoded information. Hence, the proposed scheme's UEP capability may be employed to protect the MC-encoded information with a relatively strong error correction capability, whilst employing a relatively weak error correction code to protect the DCT-encoded information. This approach may hence be expected to yield a lower degree of video reconstruction distortion than equal protection, as noted in [7-9], for example.

The rest of this chapter is outlined as follows. In Section 7.2, we propose iteratively decoded schemes, in which we opt for serially concatenating IrVLC with Trellis Coded Modulation (TCM) [129]. Furthermore, Section 7.2 additionally introduces our benchmark schemes, where IrVLC is replaced by regular VLCs having the same coding rate. The design and EXIT chart aided characterisation of these schemes is detailed in Section 7.3. In Section 7.4, we quantify the attainable performance improvements offered by the proposed IrVLC arrangements compared to the regular VLC benchmarker schemes. Furthermore, in Section 7.4 we additionally consider a Huffman coding and IrCC based benchmarker. Section 7.4 also employs a novel method of quantifying the computational complexity required for the schemes considered in order to achieve different source sample reconstruction qualities at a range of Rayleigh fading channel SNRs. This method is employed to select our preferred scheme and to characterise the benefits of UEP. Finally, we offer our conclusions in Section 7.5. 


\subsection{Overview of proposed schemes}

In this section we provide an overview of a number of serially concatenated [131] and iteratively decoded [132] joint source and channel coding schemes. Whilst the novel schemes introduced in this paper may be tailored for operating in conjunction with any inner channel codec, we opt for employing TCM [129] in each of our considered schemes. This provides error protection without any bandwidth expansion or effective bit-rate reduction by accommodating the additional redundancy by transmitting more bits per channel symbol. The choice of TCM is further justified, since A Posteriori Probability (APP) TCM Soft-In Soft-Out (SISO) decoding, similarly to APP SISO IrVLC decoding, operates on the basis of Add-CompareSelect (ACS) operations within a trellis structure. Hence, the APP SISO IrVLC and TCM decoders can share resources in systolic-array based chips, facilitating a cost effective implementation. Furthermore, we will show that TCM exhibits attractive EXIT characteristics in the proposed IrVLC context even without requiring TTCM- or BICM-style internal iterative decoding [197].

Our considered schemes differ in their choice of the outer source codec. Specifically, we consider a novel IrVLC codec and an equivalent regular VLC-based benchmarker in this role. In both cases we employ both Symbol-Based (SB) [2] and Bit-Based (BB) [90] VLC decoding, resulting in a total of four different configurations. We refer to these four schemes as the SBIrVLC-, BBIrVLC-, SBVLC- and BBVLC-TCM arrangements, as appropriate. A schematic that is common to each of these four considered schemes is provided in Figure 7.2.

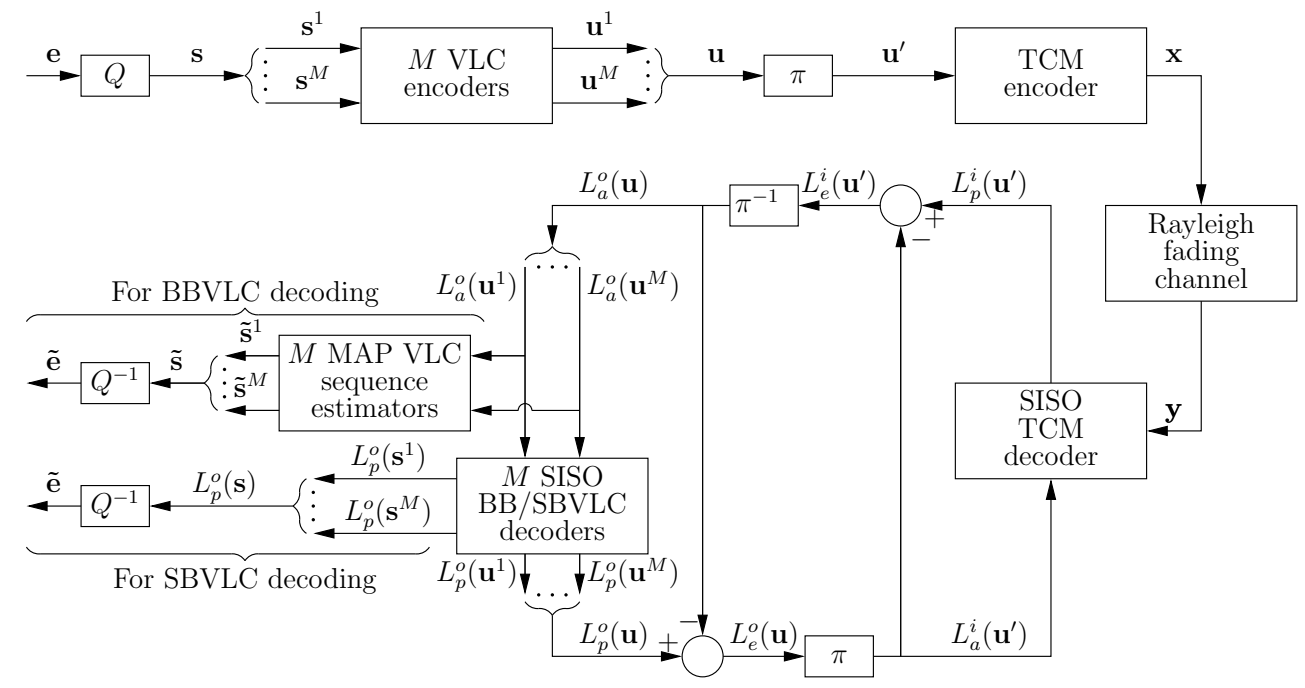

Figure 7.2: Schematic of the SBIrVLC-, BBIrVLC-, SBVLC- and BBVLC-TCM schemes. In the IrVLC schemes, the $M$ number of VLC encoders, APP SISO decoders and MAP sequence estimators are each based upon one of $N$ number of component VLC codebooks. By contrast, in the VLC benchmarkers, all of the $M$ number of VLC encoders, decoders and sequence estimators are based upon the same VLC codebook. 


\subsubsection{Joint source and channel coding}

The schemes considered are designed for facilitating the near-capacity detection of source samples received over an uncorrelated narrowband Rayleigh fading channel. We consider the case of independent identically distributed (i.i.d.) source samples, which may represent the prediction residual error that remains following the predictive coding of audio, speech, image or video information $[61,62]$, for example. Note that this was exemplified in the novel video codec of Chapter 6, in which Frame Differencing (FD) was employed, as depicted in Figure 6.1. A Gaussian source sample distribution is assumed here, since this has widespread applications owing to the wide applicability of the central limit theorem [201]. Additionally, a zero mean and unity source sample variance was assumed, resulting in the Probability Distribution Function (PDF) shown in Figure 7.3. Note however that with the aid of suitable adaptation, the techniques proposed in this chapter may be just as readily applied to arbitrary source sample distributions.

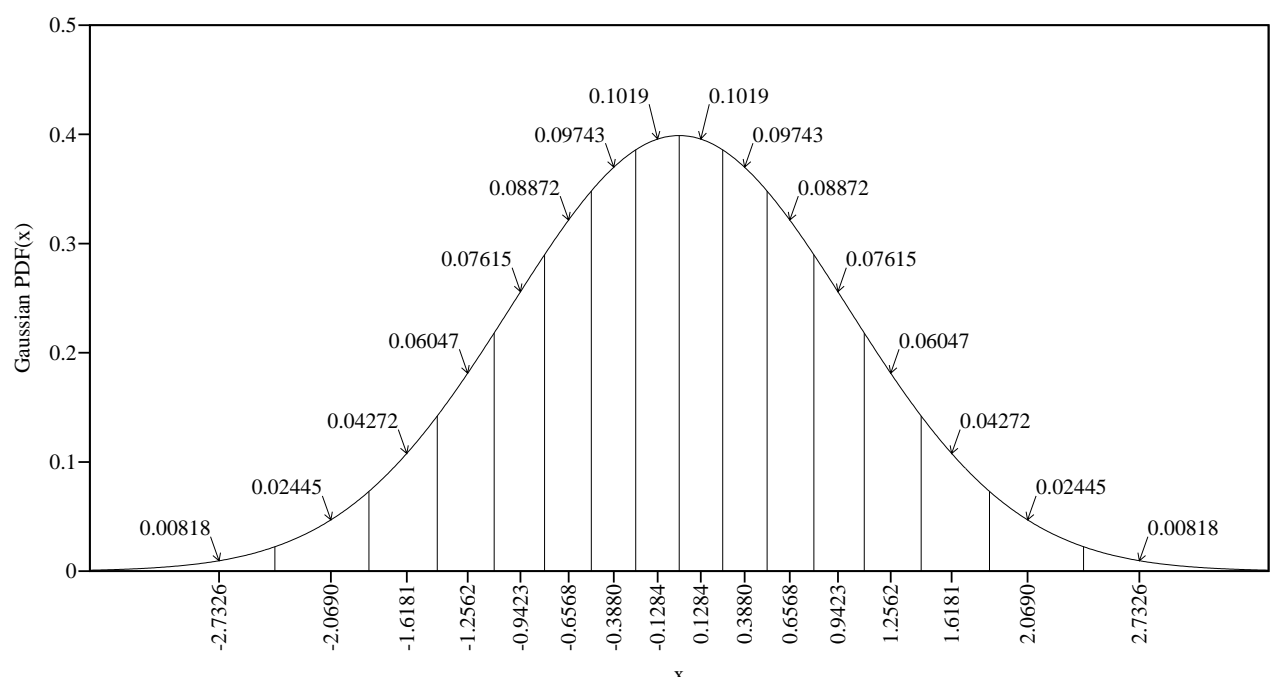

Figure 7.3: Gaussian PDF for unity mean and variance. The $\mathrm{x}$ axis is labelled with the $K=16$ Lloyd-Max quantisation levels $\left\{\hat{e}^{k}\right\}_{k=1}^{K}$ as provided in [74]. The decision boundaries are employed to decompose the Gaussian PDF into $K=16$ sections. The integral of the PDF between each pair of adjacent decision boundaries is provided.

In the block $Q$ of the transmitter depicted in Figure 7.2, each real-valued source sample of the source sample frame e is quantised [74,75] to one of the $K=16$ quantisation levels $\left\{\hat{e}^{k}\right\}_{k=1}^{K}$ provided in Figure 7.3. In each case, the selected quantisation level is that which represents the source sample with the minimum squared error. Figure 7.3 provides decision boundaries, which are located halfway between each adjacent pair of quantisation levels. Each pair of adjacent decision boundaries specifies the range of source sample values that are quantised to the quantisation level at the centre of gravity of this interval, resulting in the minimum squared error. Following quantisation, each source sample in the source sample 
frame $\mathbf{e}$ is represented by a symbol in the source symbol frame $\mathbf{s}$ that represents the index of the selected quantisation level $\hat{e}^{k}$ and has a value of $k \in[1 \ldots K]$.

Owing to the lossy nature of quantisation, distortion is imposed upon the reconstructed source sample frame ê that is obtained following inverse quantisation in the block, as described in Section ??. Note that the set of quantisation levels depicted in Figure 7.3 represents those of Lloyd-Max quantisation [74,75]. This employs the K-means algorithm [98] to search for the set of quantisation levels that minimises the expected quantisation distortion. In the case of the quantisation levels seen in Figure 7.3, the expected Signal to Quantisation Noise Ratio (SQNR) is about $20 \mathrm{~dB}$. Note however that again, with the aid of suitable adaptation, the techniques advocated in this chapter may be just as readily applied to arbitrary quantisers.

Also note that Lloyd-Max quantisation results in a large variation in the occurrence probabilities of the resultant source symbol values. These occurrence probabilities are given by integrating the source PDF between each pair of adjacent decision boundaries, resulting in the values provided in Figure 7.3. These source symbol values' occurrence probabilities $\{P(k)\}_{k=1}^{K}$ are repeated in Table 7.1 and can be seen to vary by more than ander of magnitude. These probabilities correspond to the varying source symbol informations $\left\{-\log _{2}(P(k))\right\}_{k=1}^{K}$ provided in Table 7.1, motivating the application of VLC and giving a source entropy of $E=3.77$ bits per source symbol, according to (7.2).

In the transmitter of the proposed scheme, the Lloyd-Max quantised source symbol frame $\mathbf{s}$ is decomposed into $M=300$ sub-frames $\left\{\mathbf{s}^{m}\right\}_{m=1}^{M}$, as shown in Figure 7.2. In the case of the SBIrVLC- and SBVLC-TCM schemes, this decomposition is necessary for the sake of limiting the computational complexity of VLC decoding, since the number of transitions in the symbol-based VLC trellis is inversely proportional to the number of sub-frames in this case [2], as described in Section ??. We opt for employing the same decomposition of the source symbol frames into sub-frames in the case of the BBIrVLC- and BBVLC-TCM schemes for the sake of ensuring that we make a fair comparison with the SBIrVLC- and SBVLC-TCM schemes. This is justified, since the decomposition considered benefits the performance of the BBIrVLC- and BBVLC-TCM schemes, as will be detailed below. Each source symbol sub-frame $\mathbf{s}^{m}$ comprises $J=100$ source symbols. Hence, the total number of source symbols in a source symbol frame becomes $M \cdot J=30000$. As described above, each Lloyd-Max quantised source symbol in the sub-frame $\mathbf{s}^{m}$ has a $K$-ary value $s_{j}^{m} \in[1 \ldots K]$, where we have $j \in[1 \ldots J]$.

As described in Section 7.1, we employ $N$ number of component VLC codebooks to encode the source symbols, where we opted for $N=15$ for the SBIrVLC and BBIrVLC schemes and $N=1$ for the regular SBVLC and BBVLC schemes. Each Lloyd-Max quantised source symbol sub-frame $\mathbf{s}^{m}$ is VLC-encoded using a single component VLC codebook VLC $^{n}$, where we have $n \in[1 \ldots N]$. In the case of the SBIrVLC and BBIrVLC schemes, the particular fraction $C^{n}$ of the set of source symbol sub-frames that is encoded by the specific component VLC codebook $\mathbf{V L C}^{n}$ is fixed and will be derived in Section 7.3. The specific Lloyd-Max quantised source symbols having the value of $k \in[1 \ldots K]$ and encoded by the specific component VLC codebook $\mathbf{V L C}{ }^{n}$ are represented by the codeword $\mathbf{V L C}{ }^{n, k}$, which has a length of $I^{n, k}$ bits. The $J=100 \mathrm{VLC}$ codewords that represent the $J=100$ Lloyd-Max quantised source symbols in each source symbol sub-frame $\mathbf{s}^{m}$ are concatenated 


\begin{tabular}{|r|c|c|c|r|}
\hline$k$ & $P(k)$ & $-\log _{2}(P(k))$ & $I^{k}$ & \multicolumn{1}{|c|}{ Huff $^{k}$} \\
\hline \hline 1 & 0.0082 & 6.93 & 7 & 0000000 \\
\hline 2 & 0.0244 & 5.35 & 6 & 000001 \\
\hline 3 & 0.0427 & 4.55 & 5 & 00010 \\
\hline 4 & 0.0605 & 4.05 & 4 & 0010 \\
\hline 5 & 0.0762 & 3.72 & 4 & 0100 \\
\hline 6 & 0.0887 & 3.49 & 4 & 0110 \\
\hline 7 & 0.0974 & 3.36 & 3 & 101 \\
\hline 8 & 0.1019 & 3.29 & 3 & 110 \\
\hline 9 & 0.1019 & 3.29 & 3 & 111 \\
\hline 10 & 0.0974 & 3.36 & 3 & 100 \\
\hline 11 & 0.0887 & 3.49 & 4 & 0111 \\
\hline 12 & 0.0762 & 3.72 & 4 & 0101 \\
\hline 13 & 0.0605 & 4.05 & 4 & 0011 \\
\hline 14 & 0.0427 & 4.55 & 5 & 00011 \\
\hline 15 & 0.0244 & 5.35 & 5 & 00001 \\
\hline 16 & 0.0082 & 6.93 & 7 & 0000001 \\
\hline
\end{tabular}

Table 7.1: The probabilities of occurrence $P(k)$ and informations $-\log _{2}(P(k))$ of the $K=16$ source symbol values $k \in[1 \ldots K]$ that result from the Lloyd-Max quantisation of Gaussian distributed source samples. The corresponding source symbol entropy is $E=3.77$ bits per source symbol, according to (7.2). Also provided is the composition of the $K=16$ codewords in the corresponding Huffman codebook Huff $=\left\{\mathbf{H u f f}^{k}\right\}_{k=1}^{K}$ [65], having the codeword lengths $\left\{I^{k}\right\}_{k=1}^{K}$. According to (7.1), the average Huffman codeword length is $L($ Huff $)=3.81$ bits per source symbol, which corresponds to a Huffman coding rate of $R_{\text {Huff }}=0.99$, according to (7.3).

to provide the transmission sub-frame

$$
\mathbf{u}^{m}=\left\{\mathbf{V L C} \mathbf{C}^{n, s_{j}^{m}}\right\}_{j=1}^{J} .
$$

Owing to the variable lengths of the VLC codewords, each of the $M=300$ transmission sub-frames typically comprises a different number of bits. In order to facilitate the VLC decoding of each transmission sub-frame $\mathbf{u}^{m}$, it is necessary to explicitly convey its length

$$
I^{m}=\sum_{j=1}^{J} I^{n, s_{j}^{m}}
$$

to the receiver. Furthermore, this highly error sensitive side information must be reliably protected against transmission errors. This may be achieved using a low rate block code, for example. For the sake of avoiding obfuscation, this is not shown in Figure 7.2. Note that the choice of the specific number of sub-frames $M$ in each frame constitutes a trade-off between the computational complexity of SBVLC decoding or the performance of BBVLC decoding 
and the amount of side information that must be conveyed. In Section 7.3, we shall comment on the amount of side information that is required for reliably conveying the specific number of bits in each transmission sub-frame to the decoder.

In the scheme's transmitter, the $M=300$ number of transmission sub-frames $\left\{\mathbf{u}^{m}\right\}_{m=1}^{M}$ are concatenated. As shown in Figure 7.2, the resultant transmission frame $\mathbf{u}$ has a length of $\sum_{m=1}^{M} I^{m}$ bits.

In the proposed scheme, the VLC codec is protected by a serially concatenated TCM codec. Following VLC encoding, the bits of the transmission frame $\mathbf{u}$ are interleaved using the function $\pi$ in order to provide the interleaved transmission frame $\mathbf{u}^{\prime}$, which is TCM encoded in order to obtain the channel's input symbols $\mathbf{x}$, as shown in Figure 7.2. These are transmitted over an uncorrelated narrowband Rayleigh fading channel and are received as the channel's output symbols y, as seen in Figure 7.2.

\subsubsection{Iterative decoding}

In the receiver, APP SISO TCM- and VLC-decoding are performed iteratively, as shown in Figure 7.2. Both of these decoders invoke the Bahl-Cocke-Jelinek-Raviv (BCJR) algorithm [4] on the basis of their trellises. Symbol-based trellises are employed in the case of TCM [129], SBIrVLC and SBVLC [2] decoding, whilst BBIrVLC and BBVLC decoding rely on bit-based trellises [90]. All BCJR calculations are performed in the logarithmic probability domain and using an eight-entry lookup table for correcting the Jacobian approximation in the Log-MAP algorithm [197]. The proposed approach requires only Add, Compare and Select (ACS) computational operations during iterative decoding, which will be used as our complexity measure, since it is characteristic of the complexity/area/speed trade-offs in systolic-array based chips.

As usual, extrinsic soft information, represented in the form of Logarithmic Likelihood Ratios (LLRs) [124], is iteratively exchanged between the TCM and VLC decoding stages for the sake of assisting each other's operation [131,132], as described in Section ??. In Figure 7.2, $L(\cdot)$ denotes the LLRs of the bits concerned (or the log-APPs of the specific symbols as appropriate), where the superscript $i$ indicates inner TCM decoding, while $o$ corresponds to outer VLC decoding. Additionally, a subscript denotes the dedicated role of the LLRs (or $\log$-APPs), with $a, p$ and $e$ indicating a priori, a posteriori and extrinsic information, respectively.

During each decoding iteration, the inner TCM decoder is provided with a priori LLRs pertaining to the interleaved transmission frame $L_{a}^{i}\left(\mathbf{u}^{\prime}\right)$, as shown in Figure 7.2. These LLRs are obtained from the most recent operation of the outer VLC decoding stage, as will be highlighted below. In the case of the first decoding iteration, no previous VLC decoding has been performed and hence the a priori LLRs $L_{a}^{i}\left(\mathbf{u}^{\prime}\right)$ provided for TCM decoding are all zerovalued, corresponding to a probability of 0.5 for both ' 0 ' and ' 1 '. Given the channel's output symbols $\mathbf{y}$ and the a priori LLRs $L_{a}^{i}\left(\mathbf{u}^{\prime}\right)$, the BCJR algorithm is employed for obtaining the a posteriori LLRs $L_{p}^{i}\left(\mathbf{u}^{\prime}\right)$, as shown in Figure 7.2.

During iterative decoding, it is necessary to prevent the re-use of already exploited information, since this would limit the attainable iteration gain [197], as described in Section ??. This is achieved following TCM decoding by the subtraction of $L_{a}^{i}\left(\mathbf{u}^{\prime}\right)$ from $L_{p}^{i}\left(\mathbf{u}^{\prime}\right)$, as shown in Figure 7.2. The resultant extrinsic LLRs $L_{e}^{i}\left(\mathbf{u}^{\prime}\right)$ are de-interleaved in the block 
$\pi^{-1}$ and forwarded as a priori LLRs for VLC decoding. As described in Section ??, interleaving is employed in order to mitigate correlation within the a priori LLR frames. This is necessary since the BCJR algorithm assumes that all a priori LLRs that can influence any particular decoding decision are uncorrelated.

Just as $M=300$ separate VLC encoding processes are employed in the proposed scheme's transmitter, $M=300$ separate VLC decoding processes are employed in its receiver. In parallel to the composition of the bit-based transmission frame $\mathbf{u}$ from its $M=300$ sub-frames, the a priori LLRs $L_{a}^{o}(\mathbf{u})$ are decomposed into $M=300$ sub-frames, as shown in Figure 7.2. This is achieved with the aid of the explicit side information that conveys the number of bits $I^{m}$ in each transmission sub-frame $\mathbf{u}^{m}$. Each of the $M=300$ VLC decoding processes is provided with the a priori LLR sub-frame $L_{a}^{o}\left(\mathbf{u}^{m}\right)$ and in response it generates the a posteriori LLR sub-frame $L_{p}^{o}\left(\mathbf{u}^{m}\right), m \in[1 \ldots M]$. These a posteriori LLR sub-frames are concatenated in order to provide the a posteriori LLR frame $L_{p}^{o}(\mathbf{u})$, as shown in Figure 7.2. Following the subtraction of the a priori LLRs $L_{a}^{o}(\mathbf{u})$, the resultant extrinsic LLRs $L_{e}^{o}(\mathbf{u})$ are interleaved and forwarded as a priori information to the next TCM decoding iteration.

In the case of SBIrVLC and SBVLC decoding, each of the $M=300$ VLC decoding processes additionally provides log-APPs pertaining to the corresponding source symbol subframe $L_{p}^{o}\left(\mathbf{s}^{m}\right)$. This comprises a set of $K$ number of log-APPs for each source symbol $s_{j}^{m}$ in the sub-frame $\mathbf{s}^{m}$, where $j \in[1 \ldots J]$. Each of these log-APPs provides the logarithmic probability that the corresponding source symbol $s_{j}^{m}$ has the particular value $k \in[1 \ldots K]$. In the receiver of Figure 7.2, the source symbols' log-APP sub-frames are concatenated to provide the source symbol log-APP frame $L_{p}^{o}(\mathbf{s})$. By inverse-quantising this soft information in the block $Q^{-1}$, we may obtain a frame of Minimum Mean Squared Error (MMSE) source sample estimates $\tilde{\mathbf{e}}$, which approximates the reconstructed source sample frame ê described in Section 7.2.1. More specifically, each source sample estimate is obtained by using the corresponding set of $K$ source symbol value probabilities to find the weighted average of the $K$ number of quantisation levels $\left\{\hat{e}^{k}\right\}_{k=1}^{K}$.

Conversely, in the case of BBIrVLC and BBVLC decoding, no symbol-based a posteriori output is available. In this case, each source symbol sub-frame $\mathbf{s}^{m}$ is estimated from the corresponding a priori LLR sub-frame $L_{a}^{o}\left(\mathbf{u}^{m}\right)$. This may be achieved by employing Maximum A posteriori Probability (MAP) sequence estimation operating on a bit-based trellis structure, as shown in Figure 7.2. Unlike in APP SISO SBIrVLC and SBVLC decoding, bit-based MAP sequence estimation cannot exploit the knowledge that each sub-frame $\mathbf{s}^{m}$ comprises $J=100$ source symbols. For this reason, the resultant hard decision estimate $\tilde{\mathbf{s}}^{m}$ of each source symbol sub-frame $\mathbf{s}^{m}$ may or may not contain $J=100$ source symbols. In order that we may prevent the loss of synchronisation that this would imply, source symbol estimates are removed from, or appended to the end of each source symbol sub-frame estimate $\tilde{\mathbf{s}}^{m}$ for ensuring that they each comprise exactly $J=100$ source symbol estimates. Note that it is the decomposition of the source symbol frame $\mathbf{s}$ into sub-frames that provides this opportunity to mitigate the loss of synchronisation that is associated with bit-based MAP VLC sequence estimation. Hence the decomposition of the source symbol frame $\mathbf{s}$ into sub-frames benefits the performance of the BBIrVLC- and BBVLC-TCM schemes, as mentioned above.

Following MAP sequence estimation, the adjusted source symbol sub-frame estimates $\tilde{\mathbf{s}}^{m}$ are concatenated for the sake of obtaining the source symbol frame estimate $\tilde{\mathbf{s}}$. This may be inverse-quantised in order to obtain the source sample frame estimate $\tilde{\mathbf{e}}$. Note that for the 
reconstruction of a source sample frame estimate $\tilde{\mathbf{e}}$ from a given a priori LLR frame $L_{a}^{o}(\mathbf{u})$, a higher level of source distortion may be expected in the BBIrVLC- and BBVLC-TCM schemes than in the corresponding SBIrVLC- and SBVLC-TCM schemes. This is due to the BBIrVLC- and BBVLC-TCM schemes' reliance on hard decisions as opposed to the soft decisions of the SBIrVLC- and SBVLC-TCM schemes. However, this reduced performance substantially benefits us in terms of a reduced complexity, since the bit-based VLC decoding trellis employed during APP SISO BBIrVLC and BBVLC decoding and MAP sequence estimation contains significantly less transitions than the symbol-based VLC decoding trellis of APP SISO SBIrVLC and SBVLC decoding, as described in Section ??.

In the next section we detail the design of our IrVLC scheme and characterise each of the SBIrVLC-, BBIrVLC-, SBVLC- and BBVLC-TCM schemes with the aid of EXIT chart analysis.

\subsection{Parameter design for the proposed schemes}

\subsubsection{Scheme hypothesis and parameters}

As described in Section 7.1, the SBIrVLC and BBIrVLC schemes may be constructed by employing a number of component VLC codebooks having different coding rates, each of which encodes an appropriately chosen fraction of the input source symbols. We opted for using $N=15$ component VLC codebooks $\left\{\mathbf{V L C}^{n}\right\}_{n=1}^{15}$, that were specifically designed for encoding $K=16$-level Lloyd-Max quantised Gaussian i.i.d. source samples. As shown in Figure 7.1, these $N=15$ component VLC codebooks were selected from a large number of candidates using a significant amount of 'trial-and-error' based human interaction in order to provide a suite of 'similarly-spaced' EXIT functions. More specifically, the $N=15$ component VLC codebooks comprised 13 different Variable Length Error Correcting (VLEC) designs having various so-called minimum block-, convergence- and divergence-distances as defined in Section ??, complemented by a Symmetric Reversible Variable Length Coding (SRVLC) and an Asymmetric Reversible Variable Length Coding (ARVLC) design. These codebooks were designed using Algorithms $\mathrm{C}$ and E of Section ??.

As described in Section ??, the free distance lower bound of a VLC codebook VLC ${ }^{n}$ can be calculated as

$$
\bar{d}_{\text {free }}\left(\mathbf{V L C} \mathbf{C}^{n}\right)=\min \left(d_{b_{\min }}\left(\mathbf{V L C} \mathbf{C}^{n}\right), d_{d_{\min }}\left(\mathbf{V L} \mathbf{C}^{n}\right)+d_{c_{\text {min }}}\left(\mathbf{V L C} \mathbf{C}^{n}\right)\right),
$$

where $d_{b_{\min }}\left(\mathbf{V L C} \mathbf{C}^{n}\right)$ is defined as the minimum block distance between any pair of equallength codewords in the VLC codebook $\mathbf{V L C}{ }^{n}$, whilst $d_{d_{\min }}\left(\mathbf{V L C} \mathbf{C}^{n}\right)$ and $d_{c_{\min }}\left(\mathbf{V L C} \mathbf{C}^{n}\right)$ are the minimum divergence and convergence distances between any pair of unequal-length codewords, respectively. In all codebooks, a free distance lower bound of $\bar{d}_{\text {free }}\left(\mathbf{V L C}{ }^{n}\right) \geq 2$ was employed, since this supports iterative decoding convergence to an infinitesimally low probability of error [161], as described in Section ??. The resultant average VLC codeword lengths were found to range from 3.94 to $12.18 \mathrm{bits} / \mathrm{symbol}$, according to (7.1). When compared to the source symbol entropy of $E=3.77$ bits per source symbol, these correspond to coding rates spanning the range of 0.31 to 0.96 , according to (7.3). The properties and composition of the $N=15$ component VLC codebooks $\{\mathbf{V L C}\}_{n=1}^{15}$ are summarised in Table 7.2. 


\begin{tabular}{|c|c|c|}
\hline $\mathbf{V L C}^{n}$ & Properties & Composition \\
\hline VLC $^{1}$ & (VLEC, $2,1,1,2,0.96)$ & $\begin{array}{l}6,6,5,5,4,4,3,3,3,3,4,4,5,5,6,6 \\
857 \mathrm{E} 1 \mathrm{FD} 3074 \mathrm{~A} 55133 \mathrm{~A}\end{array}$ \\
\hline $\mathrm{VLC}^{2}$ & (ARVLC, $2,1,1,2,0.91)$ & $\begin{array}{l}\text { 6,6,5,5,4,4,4,3,3,4,4,4,5,5,6,6, } \\
\text { 1EB62 4C9A1D58F6E4A1 }\end{array}$ \\
\hline VLC $^{3}$ & (SRVLC,2,1,1,2,0.86) & $\begin{array}{l}7,6,6,5,5,4,4,3,3,4,4,5,5,6,6,7 \\
7 \text { D9C24 8FCAAC0EDBC } 641\end{array}$ \\
\hline $\mathrm{VLC}^{4}$ & $($ VLEC $, 3,1,1,2,0.81)$ & $\begin{array}{l}8,7,7,6,6,5,4,2,3,3,4,5,6,7,7,8, \\
81 \mathrm{~F} 6 \mathrm{~F} 9 \mathrm{E} 86322 \mathrm{ACEDE} 0 \mathrm{E} 77 \mathrm{E}\end{array}$ \\
\hline VLC $^{5}$ & (VLEC,4,1,1,2,0.75) & $\begin{array}{l}8,8,7,6,6,5,4,2,3,4,5,6,7,7,8,8 \\
36 \mathrm{EF} 61 \mathrm{~EB} \text { BA4 4 } 17 \text { 19F5D 7E81 }\end{array}$ \\
\hline VLC $^{6}$ & (VLEC $, 2,2,1,2,0.70)$ & $\begin{array}{l}8,7,7,6,6,6,4,4,4,4,6,6,6,7,7,8, \\
\text { E6C99FCADB9035628FF0E2EA }\end{array}$ \\
\hline VLC $^{7}$ & (VLEC,3,2,1,3,0.64) & $\begin{array}{l}8,8,7,7,6,6,6,4,5,5,6,6,7,7,7,9, \\
\text { 7FDE5CD3E65403625A267AAD7C }\end{array}$ \\
\hline $\mathrm{VLC}^{8}$ & (VLEC, $3,2,2,3,0.60)$ & $\begin{array}{l}9,8,8,7,7,6,6,4,6,6,6,6,7,8,8,9, \\
\text { 696F594FCBA5A03159B3F 8B35583 }\end{array}$ \\
\hline VLC $^{9}$ & (VLEC, $5,2,2,4,0.57)$ & $\begin{array}{l}\text { 10,10,9,8,8,7,6,4,5,5,6,7,8,9,9,10, } \\
\text { 126307A57CE367501B2AAC9A69CF 9ED }\end{array}$ \\
\hline $\mathbf{V L C}^{10}$ & (VLEC, $, 4,3,2,4,0.52)$ & $\begin{array}{l}11,10,9,8,8,7,7,6,6,6,7,7,8,9,9,11, \\
\text { 1673E8F0CB2DAAA4 01F 9CC68CD55E37BF }\end{array}$ \\
\hline $\mathrm{VLC}^{11}$ & (VLEC $, 4,3,3,4,0.47)$ & $\begin{array}{l}\text { 11,11,10,9,9,8,7,6,6,7,8,8,9,10,10,12, } \\
\text { 11FA38AB9536B 72B800F 4D 67B3355A655663 }\end{array}$ \\
\hline $\mathbf{V L C}^{12}$ & (VLEC,7,3,3,6,0.43) & $\begin{array}{l}\text { 12,12,11,10,10,9,8,6,7,7,8,9,11,11,12,13, } \\
\text { 2F696B8EC5D38F 93A5007715A363233BBA2B }\end{array}$ \\
\hline $\mathrm{VLC}^{13}$ & (VLEC,5,4,3,5,0.39) & $\begin{array}{l}\text { 13,12,11,10,10,9,9,8,9,9,9,10,10,11,11,14, } \\
\text { 17455A1FFED 72B7CC9380079C4 7 9A5F32C95 } \\
\text { A4D }\end{array}$ \\
\hline $\mathrm{VLC}^{14}$ & (VLEC,9,4,4,8,0.35) & $\begin{array}{l}\text { 15,14,14,12,12,11,10,8,9,9,10,11,13,13,14,15, } \\
\text { 18DA499F59CAB71C9B55C9C003DE1361552D } \\
\text { 7ACFB4D3B }\end{array}$ \\
\hline $\mathrm{VLC}^{15}$ & (VLEC, $8,5,5,8,0.31)$ & $\begin{array}{l}\text { 16,15,15,13,13,12,12,10,10,11,12,12,14,14,15,16, } \\
\text { 31D97570AE9A5A9C6A59664D4003FE87CE53 } \\
\text { C671CE53464F3A }\end{array}$ \\
\hline
\end{tabular}

Table 7.2: Properties and composition of the 15 component VLC codebooks $\left\{\mathbf{V L C}^{n}\right\}_{n=1}^{15}$. The properties of each component VLC codebook $\mathbf{V L C}^{n}$ are provided using the format (Type, $\left.d_{b_{\min }}\left(\mathbf{V L C} \mathbf{C}^{n}\right), d_{d_{\min }}\left(\mathbf{V L C} \mathbf{C}^{n}\right), d_{c_{\min }}\left(\mathbf{V L C} \mathbf{C}^{n}\right), \bar{d}_{\text {free }}\left(\mathbf{V L C} \mathbf{C}^{n}\right), R\left(\mathbf{V L C} \mathbf{C}^{n}\right)\right)$. The composition of each component VLC codebook $\mathbf{V L C}^{n}$ is specified by providing the $K=16$ codeword lengths $\left\{I^{n, k}\right\}_{k=1}^{K}$, together with the hexadecimal representation of the ordered concatenation of the $K=16$ VLC codewords in the codebook. 
As will be detailed below, our SBIrVLC and BBIrVLC schemes were designed under the constraint that they have an overall coding rate of $R_{\mathrm{IrVLC}}=0.52$. This value was chosen, since it is the coding rate of the VLC codebook VLC ${ }^{10}$, which we employ in our SBVLC and BBVLC benchmarkers using $N=1$ codebook. This coding rate results in anerage interleaver length of $M \cdot J \cdot E / R_{\mathrm{IrVLC}}=217500$ bits for all the schemes considered. Note that this interleaver length is nearly three times longer than any of those considered in Chapter 6.

Each of the schemes considered employs the same TCM codec, having the Linear Feedback Shift Register (LFSR) schematic of Figure 6.9. As shown in Figure 6.9, the TCM encoder generates a set of four bits to represent each set of three input bits, giving a coding rate of $R_{\mathrm{TCM}}=3 / 4$. Three of the four output bits are systematic replications of the three input bits, whilst the fourth output bit is generated with the aid of the $L_{\mathrm{TCM}}=6$ modulo- 2 memory elements. Note that the TCM codec is a recursive component having an infinite impulse response, since feedback is employed in the shift register of Figure 6.9. As a result, the TCM codec supports iterative decoding convergence to an infinitesimally low probability of error [159], as is the case for our component VLC codebooks, as described above. Hence, we may expect the proposed scheme to achieve iterative decoding convergence to an infinitesimally low probability of error, provided that the channel quality is sufficiently high to create an open EXIT chart tunnel and the iterative decoding trajectory approaches the inner and outer codecs' EXIT functions sufficiently closely, as discussed in Section ??. Furthermore, Figure 6.10 provides the constellation diagram for the $M_{\mathrm{TCM}}=16$-ary set-partitioned [129] QAM scheme of the TCM codec. This was employed together with In-phase Quadraturephase (IQ)-interleaving [196] for transmission over an uncorrelated narrowband Rayleigh fading channel.

Ignoring the modest bitrate contribution of conveying the side information, the effective throughput of the schemes considered is $\eta=R_{\mathrm{IrVLC}} \cdot R_{\mathrm{TCM}} \cdot \log _{2}\left(M_{\mathrm{TCM}}\right)=1.56$ bits per channel use. This implies that iterative decoding convergence to an infinitesimally low probability of error cannot be achieved when channel capacities of less than 1.56 bits per channel use [24] are attained at low $E_{c} / N_{0}$ values, where $E_{c}$ is the transmit energy per Rayleigh fading channel use and $N_{0}$ is the average noise energy. Note that the uncorrelated narrowband Rayleigh fading channel's capacity for 16QAM is less than 1.56 bits per channel use for $E_{b} / N_{0}$ values below $2.6 \mathrm{~dB}$ [116], where $E_{b}=E_{c} / \eta$ is the transmit energy per bit of source entropy. Given this point on the corresponding channel capacity versus $E_{b} / N_{0}$ function, we will be able to quantify how closely the proposed schemes may approach this ultimate limit.

Recall from Section 7.2 that it is necessary to convey the length of each transmission sub-frame $\mathbf{u}^{m}$ to the receiver in order to facilitate its VLC decoding. The amount of side information required may be determined by considering the range of transmission sub-frame lengths that can result from VLC encoding using each of the $N=15$ component codebooks. When all $J=100$ source symbols in a particular source symbol sub-frame $\mathbf{s}^{m}$ are represented by the codeword from the component VLC codebook $\mathbf{V L C}{ }^{n}$ having the maximal length $\max _{k \in[1 \ldots K]} I^{n, k}$, a maximal transmission sub-frame length of

$$
I_{\max }^{n}=J \cdot \max _{k \in[1 \ldots K]} I^{n, k}
$$


results. Similarly, a minimal transmission sub-frame length of

$$
I_{\min }^{n}=J \cdot \min _{k \in[1 \ldots K]} I^{n, k}
$$

results, when all source symbols are represented by the minimal length VLC codeword. A transmission sub-frame $\mathbf{u}^{m}$ encoded using the component VLC codebook VLC ${ }^{n}$ will therefore have one of $\left(I_{\max }^{n}-I_{\min }^{n}+1\right)$ number of lengths in the range $I^{m} \in\left[I_{\min }^{n} \ldots I_{\max }^{n}\right]$. Hence, the length of the transmission sub-frame $I^{m}$ can be represented using a fixed-length codeword comprising $\left\lceil\log _{2}\left(I_{\max }^{n}-I_{\min }^{n}+1\right)\right\rceil$ number of bits. When considering the VLC codeword lengths provided in Table 7.2, it was found for all schemes that a single 10-bit fixed-length codeword of side information is sufficient for conveying the length of each of the $M=300$ transmission sub-frames $\mathbf{u}^{m}$ in each transmission frame $\mathbf{u}$. As suggested in Section 7.2, this error sensitive side information may be protected by a low-rate block code in order to ensure its reliable transmission. Using a $R_{\text {rep }}=1 / 3$-rate repetition code results in a total of $10 \cdot M / R_{\text {rep }}=9000$ bits of side information per frame, which represents an average of just $4 \%$ of the transmitted information, when appended to the transmission frame $\mathbf{u}$, which has an average length of $M \cdot J \cdot E / R_{\mathrm{IrVLC}}=217500$ bits for all of the schemes considered.

\subsubsection{EXIT chart analysis and optimisation}

We now consider the EXIT characteristics of the various components of our various schemes. In all cases, EXIT functions were generated using uncorrelated Gaussian distributed a priori LLRs and all mutual information measurements were made using the histogram-based approximation of the LLR PDFs [152].

In Figures 7.4 and 7.5, we provide the EXIT functions $I_{e}^{i}\left(I_{a}^{i}, E_{b} / N_{0}\right)$ of the TCM scheme for a number of $E_{b} / N_{0}$ values above the channel capacity bound of $2.6 \mathrm{~dB}$. Note that owing to its recursive nature, the APP SISO TCM decoder can be seen to achieve unity extrinsic mutual information $I_{e}^{i}$ for unity a priori mutual information $I_{a}^{i}$ [159]. Additionally, the inverted EXIT functions $I_{a}^{o, n}\left(I_{e}^{o}\right)$ plotted for the $N=15$ component VLC codebooks, together with their coding rates $R\left(\mathbf{V L C}{ }^{n}\right)$, are given in Figure 7.4 for symbol-based APP SISO VLC decoding and in Figure 7.5 for bit-based APP SISO VLC decoding. Similarly to APP SISO TCM decoding, APP SISO VLC decoding achieves unity extrinsic mutual information $I_{e}^{o}$ for unity a priori mutual information $I_{a}^{o}$ in all cases, owing to the employment of codebooks having a free distance lower bound of $\bar{d}_{\text {free }} \geq 2$ [161], as discussed in Section ??. Note that the EXIT functions obtained for symbol- and bit-based APP SISO VLC decoding are slightly different. This is because unlike the bit-based APP SISO VLC decoder, the symbolbased APP SISO VLC decoder is capable of exploiting the knowledge that there are $J=100$ source symbols in each source symbol sub-frame $\mathbf{s}^{m}$, as described in Section ??.

The inverted EXIT function of an IrVLC scheme $I_{a}^{o}\left(I_{e}^{o}\right)$ can be obtained as the appropriately weighted superposition of the $N=15$ component VLC codebooks' EXIT functions,

$$
I_{a}^{o}\left(I_{e}^{o}\right)=\sum_{n=1}^{N} \alpha^{n} I_{a}^{o, n}\left(I_{e}^{o}\right),
$$




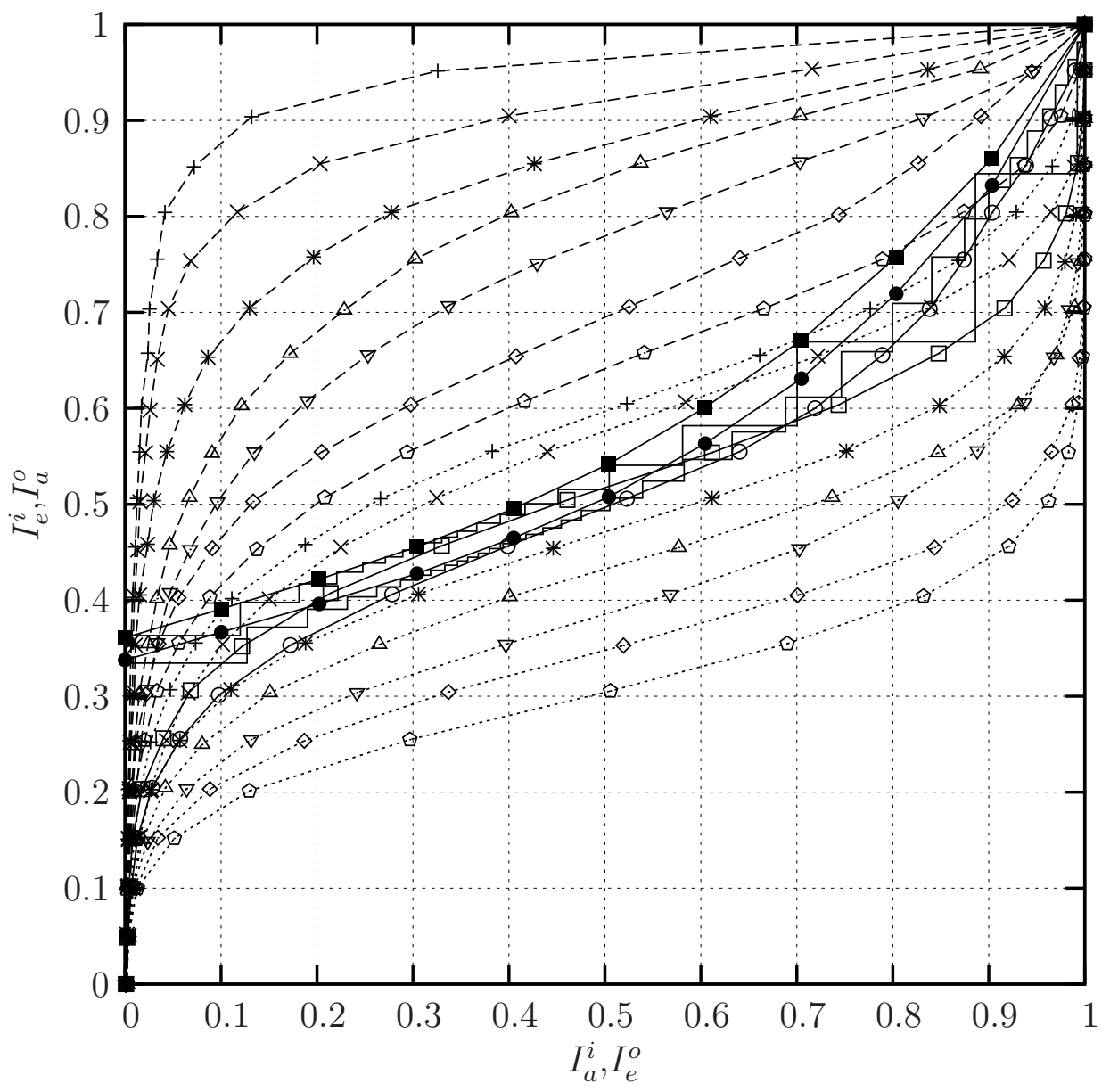

\begin{tabular}{|c|c|c|}
\hline & 00) --+-- & $\mathbf{V L C}^{10}(0.52,0.00,0.00)-\square$ \\
\hline$I^{2}$ & 00) $--x--$ & VLC $^{11}(0.47,0.65,0.72) \cdots *$ \\
\hline & 0) -- - - & $\mathbf{V L C}^{12}(0$ \\
\hline $\mathrm{LC}$ & 0) $--\Delta--$ & $\mathbf{V L C} \mathbf{C}^{13}(0$ \\
\hline $\mathrm{T}$ & 0) $--\nabla--$ & $\mathbf{V L C}^{14}(0.35,0.00,0.00) \cdots \diamond$ \\
\hline $\mathrm{LC}^{6}$ & $--\diamond--$ & VLC $^{15}$ \\
\hline$C^{7}$ & ) - - -- & $\mathrm{SBI}_{1}$ \\
\hline & & \\
\hline & $7,0.00,0.00)$ & \\
\hline
\end{tabular}

Figure 7.4: Inverted VLC EXIT functions, which were obtained using symbol-based APP SISO VLC decoding. The inverted EXIT function is provided for the corresponding SBIrVLC arrangement, together with TCM EXIT functions for a number of $E_{b} / N_{0}$ values. Decoding trajectories are provided for the SBIrVLC-TCM scheme at a channel $E_{b} / N_{0}$ value of $3.2 \mathrm{~dB}$, as well as for the SBVLC-TCM scheme at a channel $E_{b} / N_{0}$ value of $3.7 \mathrm{~dB}$. Inverted VLC EXIT functions are labelled using the format $\mathbf{V L C} \mathbf{C}^{n}\left(R\left(\mathbf{V L C} \mathbf{C}^{n}\right), C_{S B}^{n}, \alpha_{S B}^{n}\right)$. 


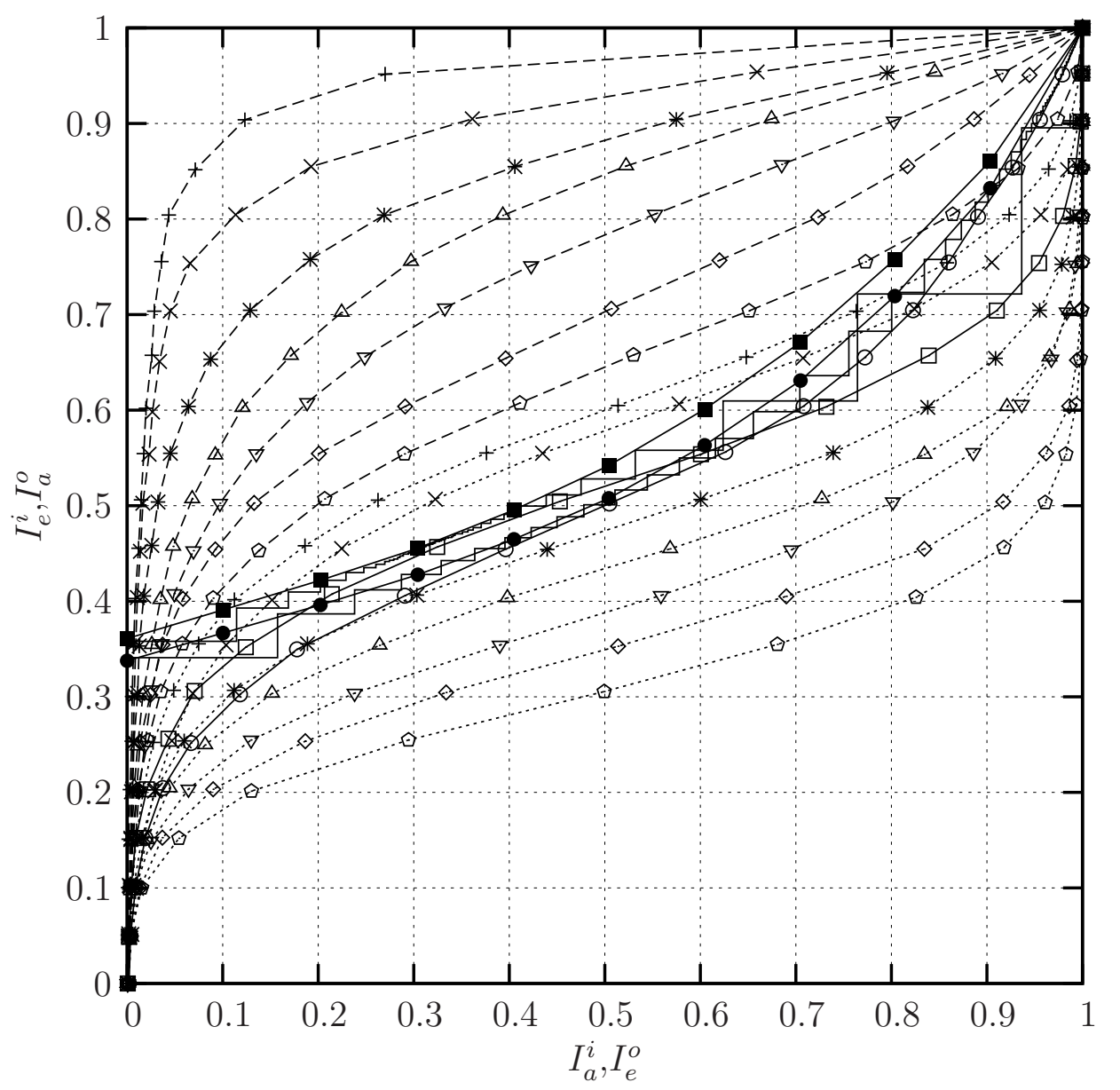

\begin{tabular}{|c|c|c|}
\hline $\mathrm{C}^{1}$ & $.00,0.00)--+--$ & $\mathbf{V L C}^{10}(0.52,0$. \\
\hline $\mathrm{VLC}^{2}$ & $.00,0.00)--\star--$ & $53,0.70) \cdots * *$ \\
\hline $\mathrm{LC}$ & 00) $--*$ & $0.00) \cdots . \Delta$ \\
\hline $\mathrm{LC}$ & 0) $--\Delta--$ & VLC \\
\hline $\mathrm{LC}$ & 2) $--\nabla--$ & VLC \\
\hline $\mathrm{LC}$ & $--\diamond--$ & VLC \\
\hline$C^{7}$ & ) ) --৫-- & BBI \\
\hline T & & \\
\hline IT & $0.57,0.00,0.00)$ & \\
\hline
\end{tabular}

Figure 7.5: Inverted VLC EXIT functions, which were obtained using bit-based APP SISO VLC decoding. The inverted EXIT function is provided for the corresponding BBIrVLC arrangement, together with TCM EXIT functions for a number of $E_{b} / N_{0}$ values. Decoding trajectories are provided for the BBIrVLC-TCM scheme at a channel $E_{b} / N_{0}$ value of $3.2 \mathrm{~dB}$, as well as for the BBVLC-TCM scheme at a channel $E_{b} / N_{0}$ value of $3.7 \mathrm{~dB}$. Inverted VLC EXIT functions are labelled using the format $\mathbf{V L C}^{n}\left(R\left(\mathbf{V L C} \mathbf{C}^{n}\right), C_{B B}^{n}, \alpha_{B B}^{n}\right)$. 
where $\alpha^{n}$ is the fraction of the transmission frame $\mathbf{u}$ that is generated by the specific component codebook VLC ${ }^{n}$. Note that since all of the $N=15$ component VLC codebooks' EXIT functions achieve unity extrinsic mutual information $I_{e}^{o}$ for unity a priori mutual information $I_{a}^{o}$, the same is true for the composite IrVLC EXIT function. Also note that the values of $\alpha^{n}$ are subject to the constraints

$$
\sum_{n=1}^{N} \alpha^{n}=1, \quad \alpha^{n} \geq 0 \forall n \in[1 \ldots N] .
$$

The specific fraction of source symbol sub-frames $\mathbf{s}^{m}$ that should be encoded by the specific component codebook $\mathbf{V L C}{ }^{n}$ in order that it generates a fraction $\alpha^{n}$ of the transmission frame $\mathbf{u}$, is given by

$$
C^{n}=\alpha^{n} \cdot R\left(\mathbf{V L C}^{n}\right) / R_{\mathrm{IrVLC}},
$$

where $R_{\mathrm{IrVLC}}=0.52$ is the desired overall coding rate. Again, the specific values of $C^{n}$ are subject to the constraints

$$
\sum_{n=1}^{N} C^{n}=\sum_{n=1}^{N} \alpha^{n} \cdot R\left(\mathbf{V L C}{ }^{n}\right) / R_{\mathrm{IrVLC}}=1, \quad C^{n} \geq 0 \forall n \in[1 \ldots N] .
$$

As described in Section ??, an open EXIT chart tunnel [158] can be achieved at sufficiently high channel $E_{b} / N_{0}$ values, since both the VLC and the TCM APP SISO decoders support iterative decoding convergence to unity mutual information. Hence, beneficial values of $\left\{C^{n}\right\}_{n=1}^{N}$ may be chosen by ensuring that there is an open EXIT chart tunnel between the inverted IrVLC EXIT function and the EXIT function of TCM at an $E_{b} / N_{0}$ value that is close to the channel capacity bound. This may be achieved using the iterative EXIT-chart matching process of [10] to adjust the values of $\left\{C^{n}\right\}_{n=1}^{N}$ under the constraints of (7.5) and (7.7) for the sake of minimising the error function

$$
\left\{C^{n}\right\}_{n=1}^{N}=\underset{\left\{C^{n}\right\}_{n=1}^{N}}{\operatorname{argmin}}\left(\int_{0}^{1} e(I)^{2} \mathrm{~d} I\right),
$$

where

$$
e(I)=I_{e}^{i}\left(I, E_{b} / N_{0}\right)-I_{a}^{o}(I)
$$

is the difference between the inverted IrVLC EXIT function and the EXIT function of TCM at a particular target $E_{b} / N_{0}$ value. Note that in order to ensure that the design results in an open EXIT tunnel, we must impose the additional constraint of

$$
e(I)>0 \forall I \in[0,1] .
$$

Open EXIT tunnels were found to be achievable for both the SBIrVLC- and the BBIrVLCTCM schemes at a threshold $E_{b} / N_{0}$ value of $3.1 \mathrm{~dB}$, which is just $0.5 \mathrm{~dB}$ from the channel capacity bound of $2.6 \mathrm{~dB}$. The inverted SBIrVLC EXIT function is shown in Figure 7.4, which is slightly different from the BBIrVLC EXIT function shown in Figure 7.5, owing to the slight differences in the EXIT functions obtained for bit- and symbol-based APP SISO 
decoding, as described above. The corresponding values of $C^{n}$ and $\alpha^{n}$ are provided for both the SBIrVLC- and the BBIrVLC-TCM schemes in Figures 7.4 and 7.5, respectively, and illustrated in Figures 7.6 and 7.7, respectively. Note that in the case of both the SBIrVLC- and BBIrVLC-TCM schemes, there are just three activated component VLC codebooks, which have corresponding values of $C^{n}$ and $\alpha^{n}$ that are higher than zero.

The source symbol frame $\mathbf{s}$ and the transmission frame $\mathbf{u}$ are depicted in Figures 7.6 and 7.7. Note that in both cases, the horizontal bar representing the source symbol frame $\mathbf{s}$ is $R_{\mathrm{IrVLC}}=0.52$ times as long as that representing the transmission frame $\mathbf{u}$, since an overall coding rate of $R_{\mathrm{IrVLC}}=0.52$ is employed. Each bar is decomposed into three sections, representing the three activated component VLCs, namely $\mathbf{V L C}^{5}, \mathbf{V L C}^{11}$ and $\mathrm{VLC}^{13}$ in the case of the SBIrVLC-TCM scheme and $\mathbf{V L C} \mathbf{C}^{5}, \mathbf{V L C} \mathbf{C}^{11}$ and $\mathbf{V L C}^{15}$ in the case of the BBIrVLC-TCM scheme. The length of each section corresponds to the fraction $C^{n}$ of the source symbol frame $\mathbf{s}$ or the fraction $\alpha^{n}$ of the transmission frame $\mathbf{u}$ that is coded using the associated component VLC codebook.

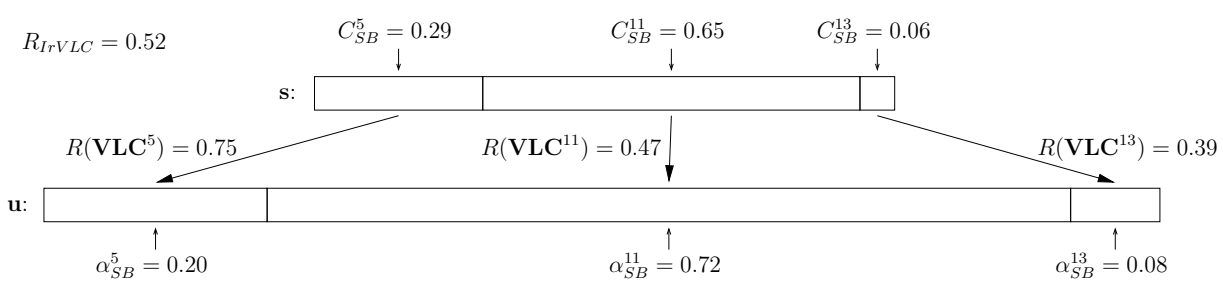

Figure 7.6: Illustration depicting the corresponding fractions of the source symbol frame $\mathrm{s}$ and the transmission frame $\mathbf{u}$ that are encoded using the three component VLC codebooks $\mathrm{VLC}^{5}, \mathbf{V L C}^{11}$ and VLC ${ }^{13}$ in the SBIrVLC-TCM scheme.

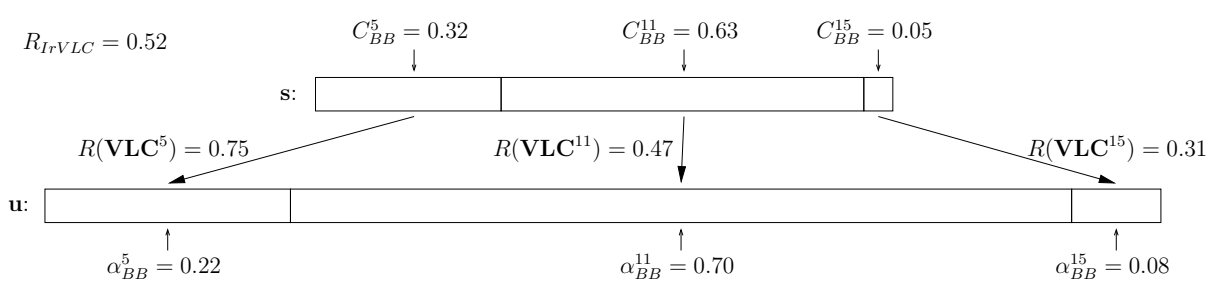

Figure 7.7: Illustration depicting the corresponding fractions of the source symbol frame $\mathrm{s}$ and the transmission frame $\mathbf{u}$ that are encoded using the three component VLC codebooks $\mathbf{V L C}^{5}, \mathbf{V L C}^{11}$ and $\mathbf{V L C}{ }^{15}$ in the BBIrVLC-TCM scheme.

In the case of the SBVLC- and BBVLC-TCM benchmarkers, an open EXIT chart tunnel between the inverted EXIT function of their only component VLC codebook VLC ${ }^{10}$ and the TCM EXIT function was only found to be achieved for $E_{b} / N_{0}$ values above a threshold value of $3.6 \mathrm{~dB}$. This $E_{b} / N_{0}$ value is $1.0 \mathrm{~dB}$ from the channel capacity bound of $2.6 \mathrm{~dB}$, a discrepancy that is twice that of the SBIrVLC- and BBIrVLC-TCM schemes' $0.5 \mathrm{~dB}$ value. We can 
therefore expect our SBIrVLC- and BBIrVLC-TCM schemes to be capable of operating significantly closer to the channel's $E_{b} / N_{0}$ capacity bound in comparison to our benchmarkers, achieving a gain of about $0.5 \mathrm{~dB}$.

\subsection{Simulation results}

In this section, we discuss our findings when communicating over an uncorrelated narrowband Rayleigh fading channel having a range of $E_{b} / N_{0}$ values above the channel capacity bound of $2.6 \mathrm{~dB}$. In all simulations, we considered the transmission of a single source sample frame e, since this comprises a sufficiently large number of samples, namely $M \cdot J=30000$.

\subsubsection{IrCC-based benchmarker}

In addition to the proposed SBIrVLC-, BBIrVLC-, SBVLC- and BBVLC-TCM schemes, in this section we also consider the operation of an additional benchmarker which we refer to as the Huffman-IrCC-TCM scheme, as depicted in the schematic of Figure 7.8. In contrast to the SBIrVLC-, BBIrVLC-, SBVLC- and BBVLC-TCM schemes of Figure 7.2, in the HuffmanIrCC-TCM scheme the transmission frame $\mathbf{u}$ is generated by both Huffman and concatenated IrCC encoding the source symbol frame $\mathrm{s}$, rather than by invoking VLC encoding.

In the Huffman-IrCC-TCM scheme, Huffman coding is employed on a sub-frame by subframe basis, as described in Section 7.2. Table 7.1 provides the composition of the $K=16$ codewords in the Huffman codebook Huff $=\left\{\mathbf{H u f f}^{k}\right\}_{k=1}^{K}$, having the codeword lengths of $\left\{I^{k}\right\}_{k=1}^{K}$. Compared to the source symbol entropy of $E=3.77$ bits per source symbol, the average Huffman codeword length is $L(\mathbf{H u f f})=3.81$ bits per source symbol and the coding rate is $R_{\text {Huff }}=0.99$, according to (7.1) and (7.3), respectively.

As shown in Figure 7.8, the frame of Huffman encoded bits $\mathbf{v}$ is protected by the $N=17$ component IrCC scheme of [175], which employs a coding memory of $L_{\mathbf{I r C C}}=4$. The inverted EXIT functions of the $N=17$ component CC codes are provided in Figure 7.9. The EXIT chart matching algorithm of [10] was employed to design the IrCC scheme. This was tailored to have an overall coding rate of $R_{\mathrm{IrCC}}=0.525$ so that the combined Huffman coding and IrCC coding rate $R_{\mathrm{Huff}} \cdot R_{\mathrm{IrCC}}=0.52$ equals that of the outer codecs in the SBIrVLC-, BBIrVLC-, SBVLC- and BBVLC-TCM schemes. Just like the SBIrVLC and BBIrVLC designs detailed in Section 7.3, an open EXIT chart tunnel was found to be achievable between the inverted IrCC EXIT function and the TCM EXIT function at an $E_{b} / N_{0}$ value of $3.1 \mathrm{~dB}$, resulting in the inverted IrCC EXIT function of Figure 7.9.

In the Huffman-IrCC-TCM receiver, iterative APP SISO IrCC and TCM decoding proceeds, as described in Section 7.2. Note that in addition to the a posteriori LLR frame $L_{p}^{o}(\mathbf{u})$ pertaining to the transmission frame $\mathbf{u}$, the APP SISO IrCC decoder can additionally provide the a posteriori LLR frame $L_{p}^{o}(\mathbf{v})$ pertaining to the frame of Huffman encoded bits $\mathbf{v}$. It is on the basis of this that bit-based MAP Huffman sequence estimation may be invoked on a sub-frame by sub-frame basis in order to obtain the source symbol frame estimate $\tilde{\mathbf{s}}$, as shown in Figure 7.8.

\subsubsection{Iterative decoding convergence performance}

For each of our schemes and for each value of $E_{b} / N_{0}$ investigated, we consider the reconstructed source sample frame ẽ and evaluate the SNR associated with the ratio of the source signal's energy and the reconstruction error energy that may be achieved following iterative decoding convergence. This relationship is plotted for each of the SBIrVLC-, BBIrVLC-, 


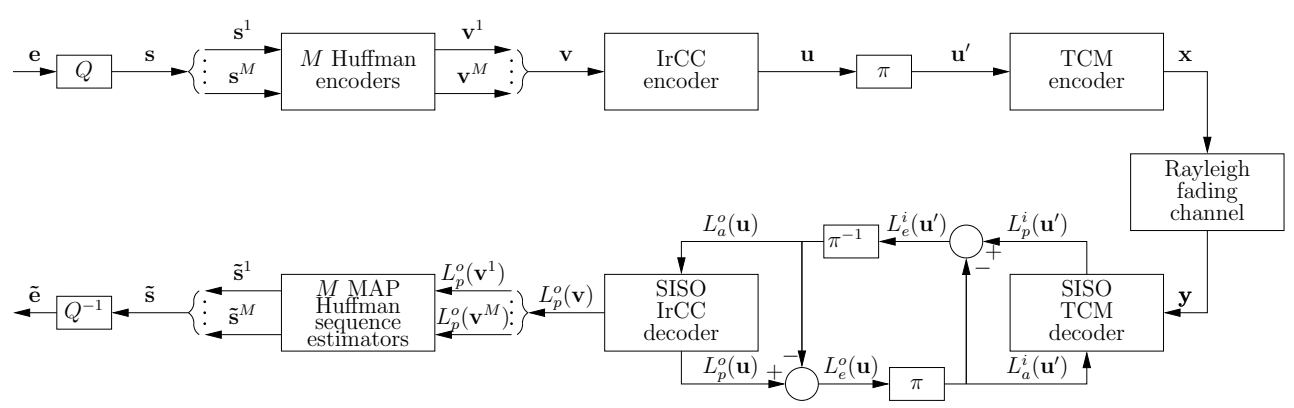

Figure 7.8: Schematic of the Huffman-IrCC-TCM scheme. All of the $M$ number of Huffman encoders and MAP sequence estimators are based upon the same Huffman coding codebook.

SBVLC- and BBVLC-TCM schemes, as well as for the Huffman-IrCC-TCM scheme, in Figure 7.10.

As shown in Figure 7.10, the source sample reconstruction SNR attained following the achievement of iterative decoding convergence increases, as the channel's $E_{b} / N_{0}$ value increases for all schemes considered. This may be explained by considering the associated EXIT chart tunnels, which gradually open and become wider as the $E_{b} / N_{0}$ value is increased from the channel's capacity bound, allowing the iterative decoding trajectory to progress further, as explained in Section ??. Note that an open EXIT chart tunnel implies that iterative decoding convergence to an infinitesimally low probability of error can be achieved, provided that the iterative decoding trajectory approaches the inner and outer codecs' EXIT functions sufficiently closely, as described in Section ??. However, it can be seen in Figure 7.10 that high source sample reconstruction SNRs were not achieved at the threshold $E_{b} / N_{0}$ values, for which open EXIT chart tunnels may be created. This is because our 217500 -bit interleaver is unable to entirely eradicate the correlation within the a priori LLR frames $L_{a}^{o}(\mathbf{u})$ and $L_{a}^{i}\left(\mathbf{u}^{\prime}\right)$, which the BCJR algorithm assumes to be uncorrelated [4]. As a result, the iterative decoding trajectory does not perfectly match with the inner and outer codecs' EXIT functions and the EXIT chart tunnel must be further widened before the iterative decoding trajectory can reach the top right hand corner of the EXIT chart, which is associated with an infinitesimally low probability of error, as described in Section ??

For sufficiently high $E_{b} / N_{0}$ values, the iterative decoding trajectory of all considered schemes was found to approach the top right hand corner of the EXIT chart, yielding source sample reconstruction SNRs of $20 \mathrm{~dB}$. As described in Section 7.2.1, this represents the infinitesimally low probability of error scenario, where quantisation noise provides the only significant degradation. As shown in Figure 7.10, source sample reconstruction SNRs of $20 \mathrm{~dB}$ may be achieved by the SBIrVLC- and BBIrVLC-TCM schemes at $E_{b} / N_{0}$ values above $3.2 \mathrm{~dB}$, which is just $0.1 \mathrm{~dB}$ from the corresponding threshold $E_{b} / N_{0}$ value of $3.1 \mathrm{~dB}$, as described in Section 7.3.2. In the case of the SBVLC- and BBVLC-TCM schemes, Figure 7.10 also shows a $0.1 \mathrm{~dB}$ discrepancy between the threshold $E_{b} / N_{0}$ value of $3.6 \mathrm{~dB}$ and the lowest $E_{b} / N_{0}$ value, for which a source sample reconstruction SNR of $20 \mathrm{~dB}$ may be achieved, namely $3.7 \mathrm{~dB}$. By contrast, Figure 7.10 shows a $0.3 \mathrm{~dB}$ discrepancy between the 


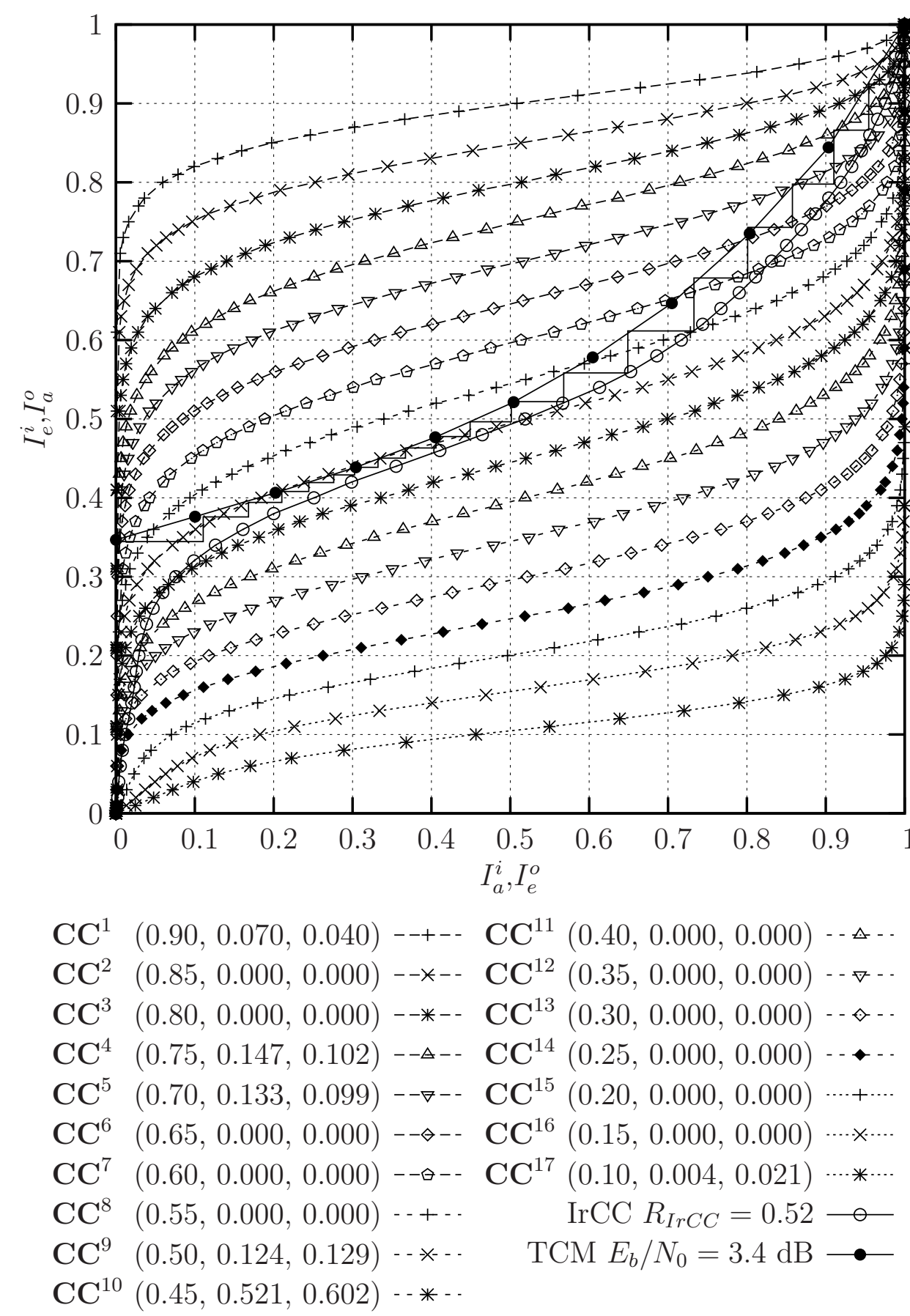

Figure 7.9: Inverted CC EXIT functions. The inverted EXIT function is provided for the corresponding IrCC arrangement, together with the TCM EXIT function corresponding to an $E_{b} / N_{0}$ value of $3.4 \mathrm{~dB}$. A decoding trajectory is provided for the Huffman-IrCC-TCM scheme at a channel $E_{b} / N_{0}$ value of $3.4 \mathrm{~dB}$. Inverted CC EXIT functions are labelled using the format $\mathbf{C C}^{n}\left(R\left(\mathbf{C C}^{n}\right), C^{n}, \alpha^{n}\right)$. 


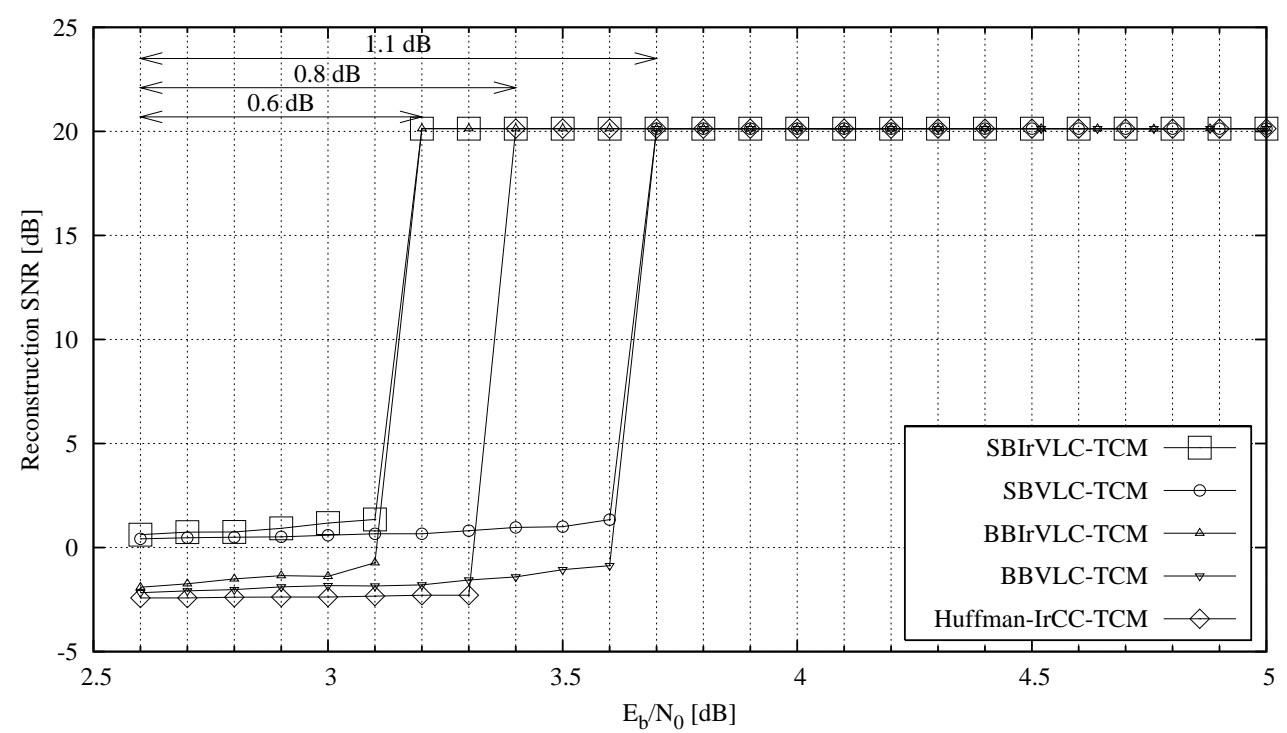

Figure 7.10: Reconstruction SNR versus $E_{b} / N_{0}$ for a Gaussian source using $K=16$ level Lloyd-Max quantisation for the SBIrVLC-, BBIrVLC-, SBVLC- and BBVLC-TCM schemes, as well as for the Huffman-IrCC-TCM scheme, communicating over an uncorrelated narrowband Rayleigh fading channel following iterative decoding convergence.

threshold $E_{b} / N_{0}$ value of $3.1 \mathrm{~dB}$ and the lowest $E_{b} / N_{0}$ value for which the Huffman-IrCCTCM scheme may achieve a source sample reconstruction SNR of $20 \mathrm{~dB}$, namely $3.4 \mathrm{~dB}$.

For each of our schemes, the iterative decoding trajectory that reaches the $(1,1)$ point of the EXIT chart at the lowest channel $E_{b} / N_{0}$ value considered is provided in either Figure 7.4, 7.5 or 7.9 , as appropriate. Note that the iterative decoding trajectories of the SBIrVLC-, BBIrVLC-, SBVLC- and BBVLC-TCM schemes approach the corresponding inner and outer EXIT functions fairly closely, facilitating iterative decoding convergence to the $(1,1)$ point of the EXIT chart at a channel $E_{b} / N_{0}$ value that is just $0.1 \mathrm{~dB}$ above the threshold value. This is in contrast to the iterative decoding trajectories of Figure 6.11, which did not exhibit a close match with the inner and outer EXIT functions, requiring an $E_{b} / N_{0}$ value that is $0.5 \mathrm{~dB}$ above the threshold value in order that the $(1,1)$ point of the EXIT chart may be reached. The improved matching of the SBIrVLC-, BBIrVLC-, SBVLC- and BBVLC-TCM schemes' iterative decoding trajectories is a benefit of employing an interleaver that is nearly three times longer than any of those employed in Chapter 6, facilitating the improved mitigation of correlation within the iteratively exchanged extrinsic information. However, the iterative decoding trajectory of the Huffman-IrCC-TCM scheme does not approach the inner and outer EXIT functions as closely as those of the SBIrVLC-, BBIrVLC-, SBVLC- and BBVLCTCM schemes. As a result, the channel's $E_{b} / N_{0}$ value must be increased by $0.3 \mathrm{~dB}$ beyond the threshold $E_{b} / N_{0}$ value before the EXIT chart tunnel becomes sufficiently wide for the iterative decoding trajectory to reach the $(1,1)$ point of the EXIT chart. This may be attributed to the APP SISO IrCC decoder's relatively high sensitivity to any residual correlation within 
the a priori LLR frame $L_{a}^{o}(\mathbf{u})$ that was insufficiently mitigated by the 217500 -bit interleaver, as will be detailed in Section 7.4.3.

\subsubsection{Interleaver length and latency}

As described in Section 7.2.2, interleaving is employed before the a priori LLR frame $L_{a}^{o}(\mathbf{u})$ is forwarded to the outer APP SISO decoder of each of the schemes considered. This is necessary, since the BCJR algorithm employed by the APP SISO decoders assumes that all a priori LLRs that can influence any particular decoding decision are uncorrelated, as described in Section ??. However, despite the employment of a long average interleaver length of 217500 bits, APP SISO IrCC decoding applied to the Huffman-IrCC-TCM scheme is still sensitive to the residual correlation within the a priori LLR frame $L_{a}^{o}(\mathbf{u})$. As a result, the Huffman-IrCC-TCM scheme suffers from a gradually eroding iterative decoding performance, when the EXIT chart tunnel is narrow, as explained above. Let us now consider the relatively high sensitivity of APP SISO IrCC decoding to the residual correlation within the a priori LLR frame $L_{a}^{o}(\mathbf{u})$ in greater detail.

In the IrCC encoder [175] of the Huffman-IrCC-TCM scheme, which employs a coding memory of $L_{\mathrm{IrCC}}=4$, each bit of the Huffman encoded frame $\mathbf{v}$ is encoded in conjunction with the preceeding $L_{\mathrm{IrCC}}=4$ bits, in order to generate an average of $1 / R_{\mathrm{IrCC}}=1.92$ bits for the transmission frame $\mathbf{u}$ [51]. Hence, each set of 1.92 bits in the transmission frame $\mathbf{u}$ is directly influenced by the values of the preceeding $L_{\mathrm{IrCC}}=4$ sets of 1.92 bits, which are each in turn directly influenced by their preceeding $L_{\mathrm{IrCC}}=4$ sets of 1.92 bits and so on, providing indirect influences. Similarly, each set of 1.92 bits in the transmission frame $\mathbf{u}$ has a direct influence upon the values of the following $L_{\mathrm{IrCC}}=4$ sets of 1.92 bits, each of which in turn has a direct influence upon their following $L_{\mathrm{IrCC}}=4$ sets of 1.92 bits and so on, providing further indirect influences. These dependencies between the sets of $1 / R_{\mathrm{IrCC}}=1.92$ bits are illustrated in Figure 7.11.

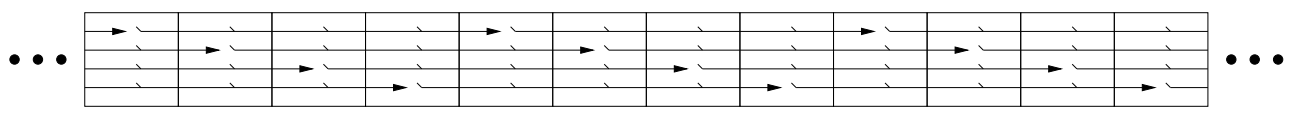

Figure 7.11: Dependencies between sets of $1 / R_{\mathrm{IrCC}}=1.92 \mathrm{IrCC}$-encoded bits, for a coding memory of $L_{\mathrm{IrCC}}=4$.

The aforementioned influences amongst the bits in the transmission frame $\mathbf{u}$ are exploited during APP SISO IrCC decoding, by employing the BCJR algorithm in order to consider the a priori LLRs in the frame $L_{a}^{o}(\mathbf{u})$ that pertain to both the preceeding and following bits of $\mathbf{u}$. However, the BCJR algorithm assumes that all a priori LLRs in the frame $L_{a}^{o}(\mathbf{u})$ that can influence a particular decoding decision are uncorrelated, as described in Section ??. Since all bits in the transmission frame $\mathbf{u}$ are either directly or indirectly influenced by each other, we could argue that APP SISO IrCC decoding is sensitive to all correlation within the a priori LLR frame $L_{a}^{o}(\mathbf{u})$. However, each set of 1.92 bits in the transmission frame $\mathbf{u}$ is only directly influenced by the values of the preceeding $L_{\mathrm{IrCC}}=4$ sets of 1.92 bits and only has direct influence upon the values of the following $L_{\mathrm{IrCC}}=4$ sets of 1.92 bits in the Huffman-IrCCTCM scheme. Hence, we can say that APP SISO IrCC decoding is only directly sensitive to correlation within the sets of $1 / R_{\mathrm{IrCC}} \times L_{\mathrm{IrCC}}+1 / R_{\mathrm{IrCC}}+1 / R_{\mathrm{IrCC}} \times L_{\mathrm{IrCC}}=17.28$ 
consecutive a priori LLRs. We may therefore conclude that the sensitivity of APP SISO IrCC decoding to correlation within the a priori LLR frame $L_{a}^{o}(\mathbf{u})$ is dependent on both the IrCC coding memory $L_{\mathrm{IrCC}}$ and on the coding rate $R_{\mathrm{IrCC}}$. Note that this implies that a shorter interleaver and latency may be afforded, provided that a higher IrCC coding rate and/or a lower memory was employed.

By contrast, during VLC encoding in the context of the SBIrVLC-, BBIrVLC-, SBVLCand BBVLC-TCM schemes, the source symbols of the source symbol frame $\mathbf{s}$ are encoded in isolation using VLC codewords having an average length of $E / R_{\mathrm{IrVLC}}=7.25$ bits, which are concatenated to provide the transmission frame $\mathbf{u}$. During APP SISO VLC decoding using the BCJR algorithm, all a priori LLRs in the frame $L_{a}^{o}(\mathbf{u})$ are considered for the sake of investigating the lengths of the VLC codewords. Despite this however, we could argue that only the a priori LLRs in the frame $L_{a}^{o}(\mathbf{u})$ that pertain to a particular VLC codeword have a direct influence upon its APP SISO decoding. We can therefore say that APP SISO VLC decoding in the SBIrVLC-, BBIrVLC-, SBVLC- and BBVLC-TCM schemes is only particularly sensitive to correlation within the sets of 7.25 consecutive a priori LLRs. Additionally, we may conclude that the sensitivity of APP SISO VLC decoding to correlation within the $a$ priori LLR frame $L_{a}^{o}(\mathbf{u})$ is dependent on only the VLC coding rate. Again, this implies that a shorter interleaver and latency may be afforded, if a higher VLC coding rate was employed.

Whilst APP SISO VLC decoding applied in the context of the SBIrVLC-, BBIrVLC-, SBVLC- and BBVLC-TCM schemes is only particularly sensitive to correlation within sets of 7.25 consecutive a priori LLRs in the frame $L_{a}^{o}(\mathbf{u})$, APP SISO IrCC decoding in the Huffman-IrCC-TCM scheme is particularly sensitive to correlation within sets of 17.28 consecutive a priori LLRs, which are about 2.4 times longer. This therefore explains the observation that the Huffman-IrCC-TCM scheme would require a longer interleaver and latency to achieve iterative decoding convergence to an infinitesimally low probability of error for channel $E_{b} / N_{0}$ values between $3.2 \mathrm{~dB}$ and $3.4 \mathrm{~dB}$.

\subsubsection{Performance during iterative decoding}

The achievement of iterative decoding convergence requires the completion of a sufficiently high number of decoding iterations. Clearly, each decoding iteration undertaken is associated with a particular computational complexity, the sum of which represents the total computational complexity of the iterative decoding process. Hence, the completion of a sufficiently high number of decoding iterations in order to achieve iterative decoding convergence may be associated with a high computational complexity. In order to quantify how this computational complexity scales as iterative decoding proceeds, we recorded the total number of ACS operations performed per source sample during APP SISO decoding and MAP sequence estimation.

Furthermore, the performance of the considered schemes was also assessed during the iterative decoding process, not only after its completion once convergence has been achieved. This was achieved by evaluating the source sample reconstruction SNR following the completion of each decoding iteration. The total computational complexity associated with this SNR was calculated as the sum of the computational complexities associated with all decoding iterations completed so far during the iterative decoding process. Clearly, as more and more decoding iterations are completed, the resultant source sample reconstruction SNR can 
be expected to increase until iterative decoding convergence is achieved. However, the associated total computational complexity will also increase as more and more decoding iterations are completed. Hence, this approach allows the characterisation of the tradeoff between reconstruction quality and computational complexity.

For each considered Rayleigh channel $E_{b} / N_{0}$ value, a set of source sample reconstruction SNRs and their corresponding computational complexities was obtained, as described above. Note that the size of these sets was equal to the number of decoding iterations required to achieve iterative decoding convergence at the particular $E_{b} / N_{0}$ value. It would therefore be possible to display the source sample reconstruction SNR versus both the $E_{b} / N_{0}$ and the computational complexity in a three-dimensional surface plot, for each of the SBIrVLC-, BBIrVLC-, SBVLC- and BBVLC-TCM schemes. For clarity however, these surfaces are projected in the direction of the source sample reconstruction SNR axis into two dimensions in the novel plot of Figure 7.12. We employ contours of constant source sample reconstruction SNR, namely $15 \mathrm{~dB}$ and $20 \mathrm{~dB}$, to parameterise the relationship between the Rayleigh fading channel's $E_{b} / N_{0}$ value and the associated computational complexity. Note that the plot of Figure 7.10 may be thought of as a cross-section through the surfaces represented by Figure 7.12, perpedicular to the computational complexity axis at $1 \cdot 10^{7}$ ACS operations per source sample. Note that this particular value of computational complexity is sufficiently high to achieve iterative decoding convergence at all values of $E_{b} / N_{0}$, in each of the considered schemes.

Note that the SBIrVLC and SBVLC decoders have a computational complexity per source sample that depends on the number of symbols in each source symbol sub-frame $\mathbf{s}^{m}$, namely $J$. This is because the number of transitions in their symbol-based trellises is proportional to $J^{2}$ [2], as described in Section ??. Hence the results provided in Figure 7.12 for the SBIrVLC- and SBVLC-TCM schemes are specific to the $J=100$ scenario. By contrast, the TCM, BBIrVLC, BBVLC and IrCC decoders have a computational complexity per source sample that is independent of the number of symbols in each source symbol sub-frame $\mathbf{s}^{m}$, namely $J$. This is because the number of transitions in their trellises is proportional to $J$ $[106,129,197]$, as described in Section ??. Hence the results for the BBIrVLC- and BBVLCTCM schemes, as well as for the Huffman-IrCC-TCM scheme, provided in Figure 7.12 are not specific for the $J=100$ case.

As shown in Figure 7.12, source sample reconstruction SNRs of up to $20 \mathrm{~dB}$ can be achieved within $0.6 \mathrm{~dB}$ of the channel's $E_{b} / N_{0}$ capacity bound of $2.6 \mathrm{~dB}$ for the SBIrVLCand BBIrVLC-TCM schemes, within $1.1 \mathrm{~dB}$ for the SBVLC- and BBVLC-TCM schemes and within $0.8 \mathrm{~dB}$ for the Huffman-IrCC-TCM scheme. Note that these findings agree with those of the EXIT chart analysis and the asymptotic performance analysis.

\subsubsection{Complexity analysis}

We now comment on the computational complexities of the considered schemes and select our preferred arrangement.

In all considered schemes and at all values of $E_{b} / N_{0}$, a source sample reconstruction SNR of $15 \mathrm{~dB}$ can be achieved at a lower computational complexity than an SNR of $20 \mathrm{~dB}$ can, as shown in Figure 7.12. This is because a reduced number of decoding iterations is required for achieving the extrinsic mutual information value associated with a lower reconstruction quality, as stated above. However, for all considered schemes operating at high values of 


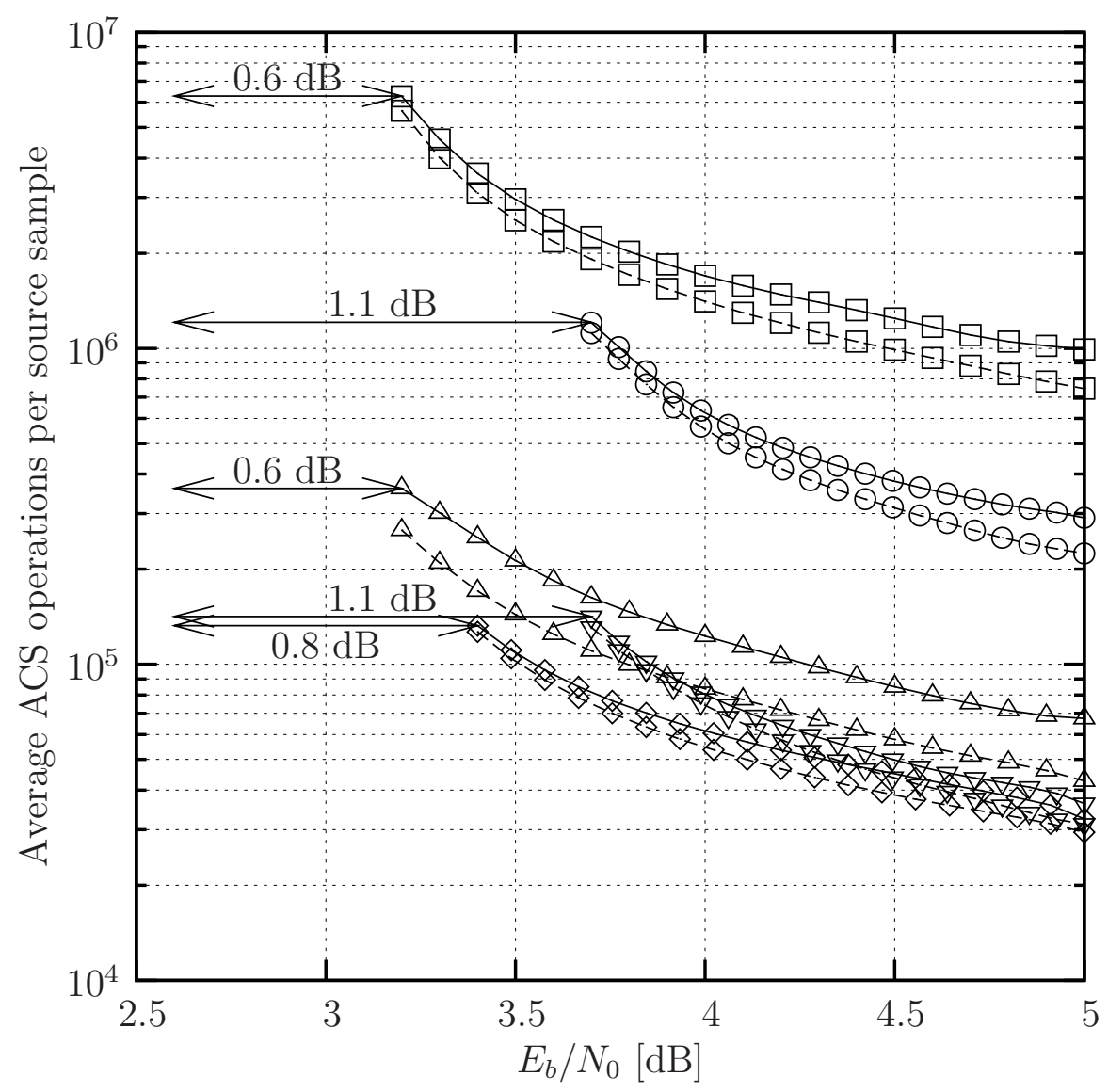

$$
\begin{aligned}
& \text { SBIrVLC-TCM 20dB } \square \quad \text { BBIrVLC-TCM 15dB --A-- } \\
& \text { SBIrVLC-TCM 15dB - - }---\quad \text { BBVLC-TCM 20dB } \square \\
& \text { SBVLC-TCM 20dB }-\quad \text { BBVLC-TCM 15dB -- } \nabla^{--} \\
& \text {SBVLC-TCM 15dB --๑-- Huffman-IrCC-TCM 20dB } \diamond
\end{aligned}
$$

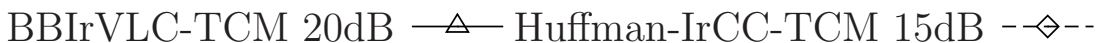

Figure 7.12: Computational complexity versus $E_{b} / N_{0}$ for a Gaussian source using $K=16$ level Lloyd-Max quantisation for the SBIrVLC-, BBIrVLC-, SBVLC- and BBVLC-TCM schemes, as well as for the Huffman-IrCC-TCM scheme, communicating over an uncorrelated narrowband Rayleigh fading channel, parameterised with the source sample reconstruction SNR. 
$E_{b} / N_{0}$, this significant $5 \mathrm{~dB}$ reduction in source sample reconstruction SNR facilitates only a relatively modest reduction of the associated computational complexity, which was between 9\% in the case of the Huffman-IrCC-TCM scheme and 36\% for the BBIrVLC-TCM scheme. Hence we may conclude that the continuation of iterative decoding until near-perfect convergence is achieved can be justified at all values of $E_{b} / N_{0}$.

Additionally, it may be seen that a given source sample reconstruction SNR may be achieved at a reduced computational complexity for all considered schemes as the $E_{b} / N_{0}$ value increases. This may be explained by the widening of the EXIT chart tunnel, as the $E_{b} / N_{0}$ value increases. As a result, less decoding iterations are required for reaching the extrinsic mutual information that is associated with a specific source sample reconstruction SNR considered.

In each of the considered schemes it was found that VLC and CC decoding is associated with a higher contribution to the total computational complexity than TCM decoding. Indeed, in the case of the SBIrVLC- and SBVLC-TCM schemes, it was found that VLC decoding accounts for about $97 \%$ of the numbers of ACS operations per source sample, having a complexity of about 32.3 times higher than that of TCM decoding. By contrast, in the BBIrVLC- and BBVLC-TCM schemes, VLC decoding accounts for only $70 \%$ of the operations, having a complexity of about 2.3 times that of TCM decoding. Similarly, CC decoding accounts for only $60 \%$ of the ACS operations in the Huffman-IrCC-TCM scheme, having a complexity of about 1.4 times that of TCM decoding.

The high complexity of the SBIrVLC and SBVLC decoders may be attributed to the specific structure of their trellises, which contain significantly more transitions than those of the BBIrVLC, BBVLC and IrCC decoders [2], as described in Section ??. As a result, the SBIrVLC- and SBVLC-TCM schemes have a complexity that is about an order of magnitude higher than that of the BBIrVLC- and BBVLC-TCM schemes, as well as the Huffman-IrCCTCM scheme, as shown in Figure 7.12. In the light of this, the employment of the SBIrVLCand SBVLC-TCM schemes cannot be readily justified.

Observe in Figure 7.12 that at high $E_{b} / N_{0}$ values, the SBIrVLC- and BBIrVLC-TCM schemes have a higher computational complexity than the corresponding SBVLC- or BBVLCTCM scheme. This is due to the influence of their low rate component VLC codebooks. These codebooks comprise codewords with many different lengths, which introduce many transitions, when represented in a trellis structure, as described in Section ??. The observed computational complexity discrepancy is particularly high in the case of the schemes that employ the symbol-based VLC trellis, owing to its particular nature. For this reason, the SBIrVLC-TCM scheme has a computational complexity that is $240 \%$ higher than that of the SBVLC-TCM scheme.

By contrast, we note that at high values of $E_{b} / N_{0}$ the BBIrVLC-TCM scheme has only about a $60 \%$ higher computational complexity than the BBVLC-TCM scheme. Similarly, the BBIrVLC-TCM scheme has only twice the computational complexity of the Huffman-IrCCTCM scheme. Coupled with the BBIrVLC-TCM scheme's ability to operate within $0.6 \mathrm{~dB}$ of the Rayleigh fading channel's $E_{b} / N_{0}$ capacity bound, we are able to identify this as our preferred arrangement. 


\subsubsection{Unequal error protection performance}

Let us now examine the UEP performance of our preferred BBIrVLC-TCM scheme. As described in Section 7.1, the UEP capability of IrVLC is manifested because different fractions of the source symbol frame $\mathbf{s}$ are encoded with different component VLC codebooks having a variety of coding rates and, hence, error correction capabilities. More specifically, the lower the coding rate of a component VLC codebook, the higher the associated potential error correction capability, as described in Section ??.

As argued above, the composite source sample reconstruction SNR was evaluated following the completion of each decoding iteration during our simulations. The total computational complexity associated with this SNR was calculated as the sum of the computational complexities associated with all decoding iterations completed so far during the iterative decoding process. These computational complexities were plotted against $E_{b} / N_{0}$ and parameterised by the source sample reconstruction SNR in Figure 7.12. Note that the composite BBIrVLCTCM ACS-complexity verses $E_{b} / N_{0}$ plots are repeated in Figure 7.13. In addition to recording the composite source sample reconstruction SNR after each decoding iteration, we also recorded the reconstruction SNRs associated with the fractions of the source sample frame e that were protected by each of the three activated component VLC codebooks VLC ${ }^{5}$, $\mathbf{V L C}^{11}$ and $\mathbf{V L C}{ }^{15}$. For each case, the associated computational complexities are plotted against $E_{b} / N_{0}$ and parameterised by the source sample reconstruction SNR in Figure 7.13.

As shown in Figure 7.13, the lower the coding rate $R\left(\mathbf{V L C}^{n}\right)$ of the component VLC codebook $\mathbf{V L C}{ }^{n}$ that is employed to protect a fraction $C_{B B}^{n}$ of the source sample frame e, the lower the computational complexity that is required to reconstruct it with a particular reconstruction SNR at a particular $E_{b} / N_{0}$ value. Indeed, at high $E_{b} / N_{0}$ values the complexity associated with reconstructing the fraction of the source sample frame $\mathbf{e}$ that is protected by the $R\left(\mathbf{V L C}{ }^{5}\right)=0.75$ coding rate component VLC codebook $\mathbf{V L C} \mathbf{C}^{5}$ is about twice as high as that associated with the $R\left(\mathbf{V L C}^{11}\right)=0.47$ coding rate component VLC codebook VLC $^{11}$. This is, in turn, about 1.5 times as high as that associated with the component VLC codebook $\mathbf{V L C} \mathbf{C}^{15}$, having a coding rate of $R\left(\mathbf{V L C} \mathbf{C}^{11}\right)=0.31$. In the scenario, where only a limited iterative decoding computational complexity can be afforded at the receiver, the fractions of the source sample frame e that are protected by the different component VLC codebooks would be reconstructed with SNRs that are commensurate with the associated coding rates, demonstrating the UEP capability of the BBIrVLC-TCM scheme.

As described in Section 7.1, each of the activated component VLC codebooks VLC ${ }^{5}$, VLC $^{11}$ and VLC $^{15}$ in the BBIrVLC-TCM scheme is employed to protect a different fraction of the source sample frame e. More specifically, the component VLC codebooks VLC ${ }^{5}$, $\mathbf{V L C}^{11}$ and $\mathbf{V L C} \mathbf{C}^{15}$ each protect a fraction $C_{B B}^{5}=0.32, C_{B B}^{11}=0.63$ and $C_{B B}^{15}=0.05$ of the source sample frame e, respectively. Note that the composite computational complexity versus $E_{b} / N_{0}$ plots depend on each of the component plots. Furthermore, we may expect the composite plots to be dominated by the components plots associated with the largest fraction of the source sample frame. Specifically, these are the component plots associated with the component VLC codebook VLC ${ }^{11}$, which is employed to protect a fraction $C_{B B}^{11}=0.63$ of the source sample frame e. However, Figure 7.13 shows that the composite plots are actually dominated by the component plots associated with the component VLC codebook VLC ${ }^{5}$, which is employed to protect only a fraction $C_{B B}^{5}=0.32$ of the source sample frame e. This may be explained as follows. 


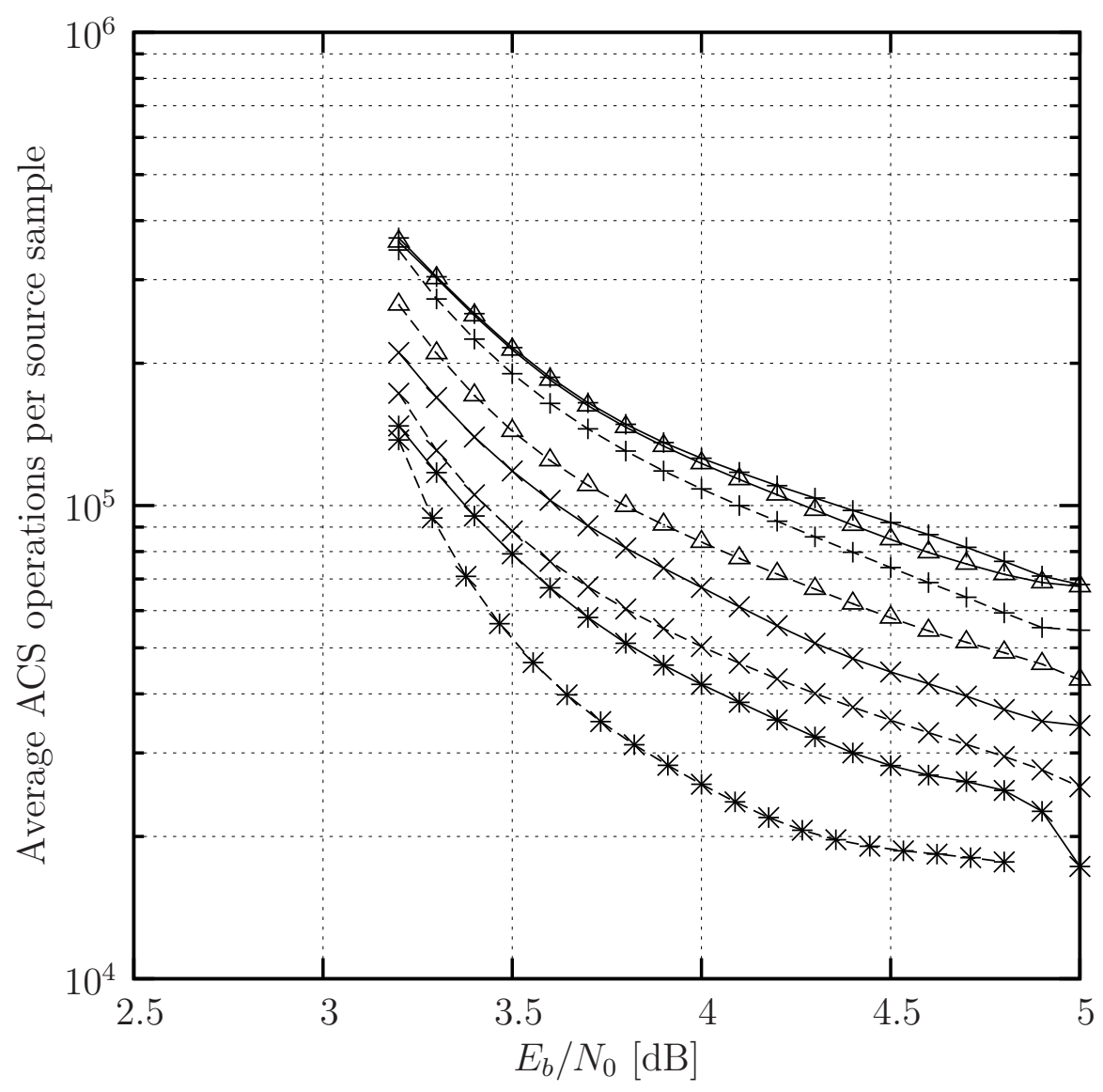

Composite 20dB $\triangle$

Composite $15 \mathrm{~dB}--\triangle--$

VLC $^{5}(0.75,0.32,0.22) 20 \mathrm{~dB}-$

VLC $^{5}(0.75,0.32,0.22) 15 \mathrm{~dB}-+--$

$\operatorname{VLC}^{11}(0.47,0.63,0.70) 20 \mathrm{~dB} \longrightarrow$

$\operatorname{VLC}^{11}(0.47,0.63,0.70) 15 \mathrm{~dB}--\rtimes--$

VLC $^{15}(0.31,0.05,0.08) 20 \mathrm{~dB}-*$

VLC $^{15}(0.31,0.05,0.08) 15 \mathrm{~dB}-*--$

Figure 7.13: Computational complexity versus $E_{b} / N_{0}$ for a Gaussian source using $K=16$ level Lloyd-Max quantisation for the BBIrVLC-TCM scheme, communicating over an uncorrelated narrowband Rayleigh fading channel, parameterised with the source sample reconstruction SNR. Separate plots are provided for the quantised source samples that are VLC encoded using each of the component VLC codebooks $V \mathbf{L} \mathbf{C}^{5}, \mathbf{V L C}^{11}$ and $\mathbf{V L C}{ }^{15}$, together the composite BBIrVLC-TCM plots of Figure 7.12. Components are labelled using the format $\mathbf{V L C} \mathbf{C}^{n}\left(R\left(\mathbf{V L C} \mathbf{C}^{n}\right), C_{B B}^{n}, \alpha_{B B}^{n}\right)$. 
A composite error-free reconstruction SNR of $20 \mathrm{~dB}$ can only be achieved if error free reconstruction is attained for all three of the fractions of the source sample frame e that are protected by the three activated component VLC codebooks $\mathbf{V L C}^{5}, \mathbf{V L C}^{11}$ and $\mathbf{V L C}^{15}$. Hence, the composite computational complexity versus $E_{b} / N_{0}$ plot that is parameterised by an error-free reconstruction SNR of $20 \mathrm{~dB}$ is dominated by that associated with the specific component VLC codebook having the weakest error correction capability, namely $\mathbf{V L C}^{5}$, as shown in Figure 7.13 and observed above. Note that the component VLC codebook VLC ${ }^{5}$ has the weakest error correction capability of the three activated codebooks, since it has the highest coding rate of $R\left(\mathbf{V L C} \mathbf{C}^{5}\right)=0.75$, as shown in Figure 7.13. This effect may also explain the domination of the composite plot that is parameterised by a reconstruction SNR of $15 \mathrm{~dB}$ corresponding to that associated with the component VLC codebook VLC ${ }^{5}$, despite a relatively low fraction of $C^{5}=0.32$ being protected by this codebook.

\subsection{Summary and Conclusions}

In this chapter, we have investigated the application of IrVLCs for EXIT chart matching. This was prompted by the observation that the serially concatenated video transmission scheme of Chapter 6 could have facilitated operation at channel $E_{b} / N_{0}$ values that are closer to the capacity bound, if the EXIT functions of its inner and outer codecs were better matched. More specifically, this would have facilitated the creation of an open EXIT chart tunnel at nearcapacity $E_{b} / N_{0}$ values, implying that iterative decoding convergence to an infinitesimally low probability of error may be achieved, if the iterative decoding trajectory approaches the inner and outer codecs' EXIT functions sufficiently closely.

In analogy to IrCCs, the novel IrVLC scheme of this chapter employs a number of component VLC codebooks having different coding rates for the sake of generating particular fractions of the transmission frame, as described in Section 7.1. We demonstrated that this provides a UEP capability, which may be employed to appropriately protect the various components of audio-, speech-, image- and video-coded information, which typically have different error sensitivities. Furthermore, we showed in Figures 7.4 and 7.5 that the composite inverted IrVLC EXIT function is given by a weighted average of the inverted EXIT functions of the individual component VLC codebooks, where each weight is given by the specific fraction of the transmission frame that is generated by the corresponding component. Finally, we demonstrated that this inverted IrVLC EXIT function may be shaped to match the EXIT function of a serially concatenated TCM codec using the EXIT chart matching algorithm of [10].

It was noted that an IrVLC scheme's component VLC codebooks should have a suite of widely varying inverted EXIT functions in order that accurate EXIT chart matching can be performed. Hence, a significant amount of 'trial-and-error' based human interaction was required in order to select our component VLC codebooks. In Chapter 8 we shall therefore propose and characterise an efficient technique for designing high quality suites of component VLC codebooks that does not require 'trial-and-error' based human interaction. In addition to this, Chapter 8 will investigate the relationship between the suite of component VLC codebooks and the resultant IrVLC EXIT chart matching accuracy. Furthermore, the application of IrVLCs for EXIT chart matching will be further explored in Chapter 9, where the EXIT functions of IrVLCs and of novel Irregular Unity Rate Codes (IrURCs) will be 
jointly matched to each other, facilitating the creation of an open EXIT chart tunnel at channel $E_{b} / N_{0}$ values that are even closer to the channel's capacity bound.

During the EXIT chart matching investigations of this chapter, an open EXIT chart tunnel was created in Figures 7.4 and 7.5 for the IrVLC-TCM schemes considered and in Figure 7.9 for the Huffman-IrCC-TCM benchmarker for channel $E_{b} / N_{0}$ values above a threshold of $3.1 \mathrm{~dB}$. This is just $0.5 \mathrm{~dB}$ from the Rayleigh fading channel's $E_{b} / N_{0}$ capacity bound of $2.6 \mathrm{~dB}$, which corresponds to our schemes' effective throughput of 1.56 bits per channel use. By contrast, an open EXIT chart tunnel was only facilitated in Figures 7.4 and 7.5 for the conventional regular VLC-TCM benchmarkers for the increased channel $E_{b} / N_{0}$ values in excess of a threshold of $3.6 \mathrm{~dB}$, which is $1.0 \mathrm{~dB}$ from the channel's capacity bound, corresponding to twice the discrepancy of the IrVLC-TCM schemes. Note that the abovementioned discrepancy of the VLC-TCM benchmarkers is similar to the $1.29 \mathrm{~dB}$ discrepancy of the VDVQ/RVLC-TCM scheme of Chapter 6, which also does not employ irregular coding techniques.

The iterative decoding performance and computational complexity of the considered schemes was investigated in a novel context using plots of the computational complexity required to achieve particular source sample reconstruction SNRs as a function of the channel's $E_{b} / N_{0}$ value in Figure 7.12. Recall that we observed that the iteratively decoded video transmission scheme of Chapter 6 would have been capable of achieving iterative decoding convergence to an infinitesimally low probability of error at channel $E_{b} / N_{0}$ values that are closer to the threshold at which an open EXIT chart tunnel can be achieved, if a longer interleaver was employed. This prompted the consideration of an interleaver having a length of 217500 bits in this chapter, which is nearly three times longer than any of those considered in Chapter 6. Indeed, it was found that the IrVLC- and VLC-TCM schemes were capable of achieving a high-quality source sample reconstruction within $0.1 \mathrm{~dB}$ of the threshold channel $E_{b} / N_{0}$ values, which were the lowest values at which an open EXIT chart tunnel was achieveable.

However, in the case of the Huffman-IrCC-TCM scheme, high quality source sample reconstruction was only achieveable for channel $E_{b} / N_{0}$ values above $3.4 \mathrm{~dB}$, which is $0.3 \mathrm{~dB}$ above the threshold at which an open EXIT chart tunnel may be achieved. This was explained in Section 7.4.3 by the relatively high sensitivity of the APP SISO IrCC decoder to any residual correlation within the a priori LLRs, that was insufficiently mitigated by the 217500 bit interleaver. This resulted in a poor match between the iterative decoding trajectory and the inverted IrCC EXIT function. More specifically, we concluded that an APP SISO IrCC decoder's sensitivity to this correlation depends on both its coding rate and, in particular, its coding memory, which had the relatively high value of $L_{\mathrm{IrCC}}=4$ in the $\mathrm{IrCC}$ scheme considered. We additionally concluded that an APP SISO VLC decoder's sensitivity to the aforementioned correlation depends only on its coding rate and that shorter interleavers and latencies could be afforded, if a higher coding rate was employed. Note that the effect of the VLC coding rate upon its sensitivity to correlation within the a priori LLRs frame will be investigated in Chapter 8 , whilst the effect of the interleaver length upon iterative decoding shall be investigated in greater detail in Chapter 9.

Recall that the outer APP SISO video decoder of the iteratively decoded video transmission scheme of Chapter 6 operated on the basis of the trellis outlined in Section 6.3.4, which is reminiscent of the symbol-based VLC trellis of [2]. This was the rationale of why 
this video transmission scheme was associated with a higher computational complexity than the benchmarkers, which employed the bit-based VLC trellis of [90] as the basis of their outer APP SISO decoders. Hence, in this chapter we characterised the computational complexity associated with employing both symbol- and bit-based trellises as the basis of APP SISO VLC decoding. In both cases, we concluded that the computational complexity associated with continuing iterative decoding until convergence is achieved is justified owing to the significantly improved reconstruction quality that results. As predicted in Chapter 6, the schemes that employed bit-based trellises for APP SISO VLC decoding were found to achieve iterative decoding convergence with a significantly lower computational complexity than the schemes employing symbol-based trellises in Figure 7.12.

Owing to its reduced iterative decoding computational complexity, the IrVLC-TCM scheme employing the bit-based VLC trellis as the basis of APP SISO VLC decoding was identified as our preferred arrangement in Section 7.4.5. Additionally, for this reason, only bit-based trellises will be employed as the basis of APP SISO VLC decoding in Chapters 8 and 9. Note that in this chapter, the source symbol frame was decomposed into $M=300$ sub-frames in order that the computational complexity associated with the symbol-based VLC trellis could be limited. However, explicit side information was required in order to convey the length of each of the corresponding transmission sub-frames to the receiver, resulting in a tradeoff between the computational complexity associated with the symbol-based VLC trellis and the amount of side information required. Indeed, in all IrVLC-TCM parameterisations considered, the required side information was found to account for $4 \%$ of the total information conveyed in Section 7.3. In this chapter, the source symbol frame was also decomposed into $M=300$ sub-frames, when the bit-based VLC trellis was employed, in order that a fair comparison could be obtained. However, since Chapters 8 and 9 will only consider the employment of the bit-based VLC trellis rather than the symbol-based VLC trellis, a significant reduction in the amount of required side information will be achieved by employing a single source symbol sub-frame per activated component VLC codebook. 


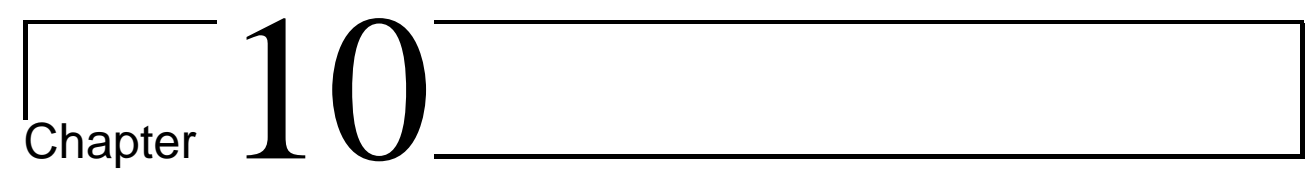

\section{Conclusions and Future Research}

\subsection{Chapter 1: Introduction}

This chapter constitutes the general background of our studies throughout the book. More specifically, a brief overview of the literature of source encoding and soft source decoding was presented in Section ??. Then the development of iterative decoding techniques and their convergence analysis was described in Section ??. Furthermore, as a special case of iterative decoding, joint source-channel decoding was introduced and the main contributions to the open literature were summarised in Section ??. Finally, the organisation of the book was described in Section ??, while our novel contributions were highlighted in Section ??.

\subsection{Chapter 1: Information Theory Basics}

In this chapter we focussed our attention on the basic Shannonian information transmission scheme and highlighted the differences between Shannon's theory valid for ideal source and channel codecs as well as for Gaussian channels and its ramifications for Rayleigh channels. We also argued that practical finite-delay source codecs cannot operate at transmission rates as low as the entropy of the source. However, these codecs do not have to operate losslessly, since perceptually unobjectionable distortions can be tolerated. This allows us to reduce the associated bit rate.

Since wireless channels exhibit bursty error statistics, the error bursts can only be randomized with the aid of infinite-length channel interleavers, which are not amenable to realtime interactive multimedia communications. Although with the advent of high-delay turbo channel codecs it is possible to operate near the Shannonian performance limits over Gaussian channels, over bursty and dispersive channels different information-theoretical channel capacity limits apply.

We considered the entropy of information sources both with and without memory and highlighted a number of algorithms, such as the Shannon-Fano, the Huffman and run-length coding algorithms, designed for the efficient encoding of sources exhibiting memory. This 
was followed by considering the transmission of information over noise-contaminated channels leading to Shannon's channel coding theorem. Our discussions continued by considering the capacity of communications channels in the context of the Shannon-Hartley law. The chapter was concluded by considering the ramifications of Shannon's messages for wireless channels.

\subsection{Chapter ??: Sources and Source Codes}

Chapter ?? commenced with the description of general source models, among which a memoryless source model having a known finite alphabet such as that described in Section ?? was used throughout the monograph. Then various source codes such as Huffman codes, RVLCs and VLEC codes were introduced in Section ??, along with their construction methods. An important contribution of this chapter is that a generic algorithm was presented for the construction of efficient RVLCs and VLEC codes. The philosophy of our proposed algorithm is that we first construct an initial RVLC or VLEC code using existing methods such as those described in $[?, ?, ?]$, then we optimise the codeword length distribution of the resultant code length-by-length. For example, Fig. 10.1 shows the evolution of the codeword length histograms of the RVLC designed for the English Alphabet in Section ??. After 12 iterations of optimisation, the best codeword length distribution is found, resulting in a RVLC having the lowest average codeword length of $A L=4.18732$.

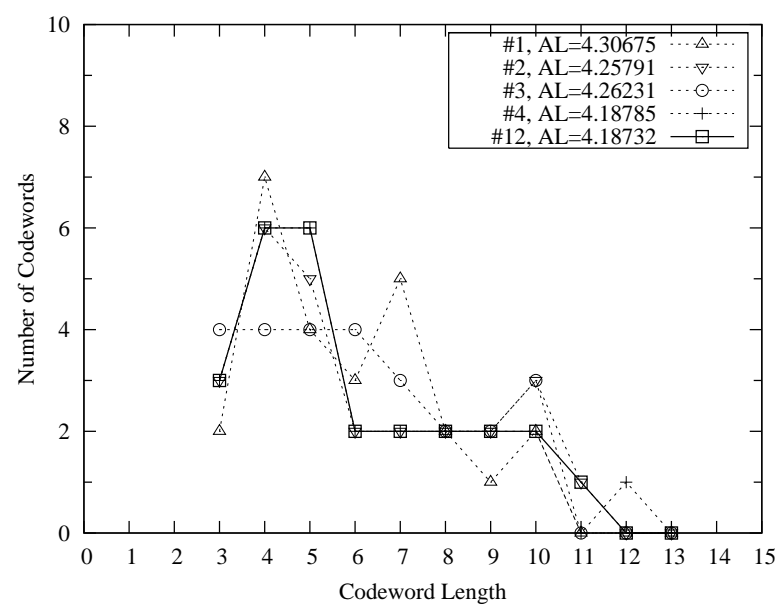

Figure 10.1: Evolution of the RVLC codeword length histograms. The RVLC is designed for the English Alphabet and its detailed construction process is described in Section ??. The codeword length distribution is optimised via a number of iterations for the sake of reducing the average codeword length.

Consequently, as shown in Table ??, Table ?? and Table ??, for a variety of memoryless sources, the proposed algorithm was capable of generating RVLCs of higher code efficiency and/or shorter maximum codeword length than the algorithms previously disseminated in the literature. Furthermore, as seen from Table ?? and Table ??, the proposed algorithm was also 
capable of constructing VLEC codes having similar code efficiency as those generated by the existing algorithm [?], but incurring a significantly lower complexity.

In Section ??, various VLC decoding methods were presented. First, the source information, such as the number of bits/symbols in the transmitted frames, and the constraints imposed by a source code and formulated in terms of the corresponding codebook were translated into a trellis representation, such as the symbol-based trellis described in Section ?? or the bit-based trellis described in Section ??. Then MAP/ML sequence estimation or MAP decoding may be performed, which were introduced in Section ?? and Section ??, respectively. It has been shown in Section ?? that trellis based soft-decoding provides an effective way of capitalising on the available information as much as possible. In general, the more information is utilised, the better the performance. This information can be explicit, such as the transmission frame length information, or implicit, such as the code constraint of a VLC. For example, soft-decoding generally outperforms hard-decoding, and the symbol-level trellis based decoding outperforms the bit-level trellis based decoding. Furthermore, as expected, VLCs having higher free distances outperform VLCs having lower free distances at the price of a reduced system throughput. Fig. 10.2 provides some quantitative results, summarising the conclusions of Section ??. It can be seen from Fig. 10.2a that soft-decision decoding significantly outperforms hard-decision decoding and the attainable $E_{b} / N_{0}$ gain improves upon increasing the VLC's free distance. Moreover, as seen from Fig. 10.2b, the performance of soft ML decoding improves upon increasing the free distance of the VLC used.

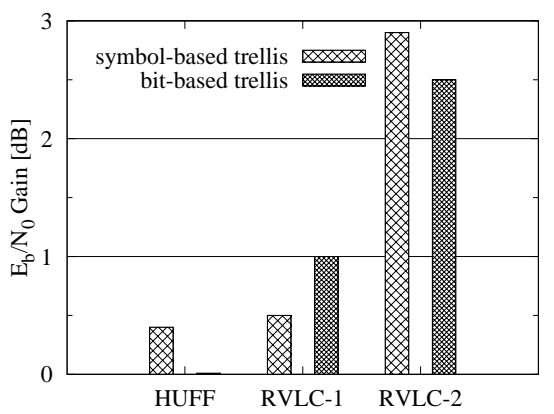

(a) Soft Decision versus Hard Decisions

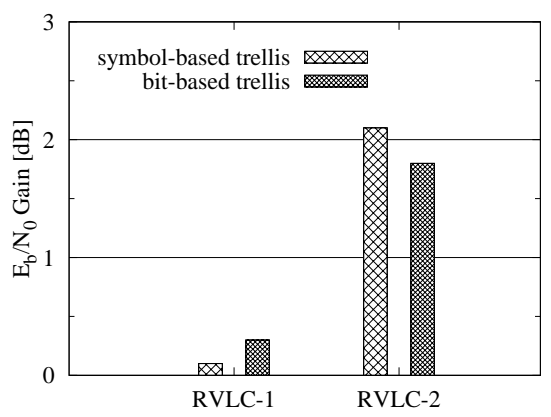

(b) Effects of different VLC Constraints

Figure 10.2: Comparison of the various VLC decoding schemes investigated in Section ??. Fig. 10.2a compares the performance of soft-decision and hard-decision decoding based schemes, where the $E_{b} / N_{0}$ gain is defined as the difference of the minimum $E_{b} / N_{0}$ values required for achieving a SER of $10^{-5}$ for transmission over AWGN channels, when using ML decoding. Fig. 10.2b demonstrates the effects of different VLC free distances, $d_{f}=1$ (RVLC-1) and $d_{f}=2$ (RVLC-2). The Huffman code (HUFF) based scheme is used as a benchmarker, where the $E_{b} / N_{0}$ gain is defined as the difference of the minimum $E_{b} / N_{0}$ values required for achieving a SER of $10^{-5}$ for transmission over AWGN channels, when using soft ML decoding. 


\subsection{Chapter ??: Iterative Source/Channel Decoding}

Chapter ?? provides an investigation of iterative source/channel decoding techniques. In this chapter, the source code, the channel code and the ISI channel are viewed as a serially concatenated system. Hence, iterative decoding may be performed, provided that the source decoder, channel decoder and the channel equaliser designed for the ISI channel are all SISO modules.

This chapter commenced with an overview of various concatenated schemes, as described in Section ??. Then a SISO APP decoding algorithm was introduced in Section ??. This algorithm provides a general description of any trellis-based APP decoding/detection scheme, which can be applied to source decoding, channel decoding and channel equalisation. Hence it constitutes the core module of iterative decoding schemes.

EXIT charts were introduced in Section ??. The mutual information between the data bits at the transmitter and the soft values at the receiver was used for characterising the decoding behaviour of a SISO APP module, resulting in the so-called EXIT functions. A histogrambased algorithm and its simplified version were introduced in Section ?? in order to evaluate the EXIT functions of a SISO APP module, followed by several examples of typical EXIT functions of SISO APP modules embedded in different positions of a concatenation scheme.

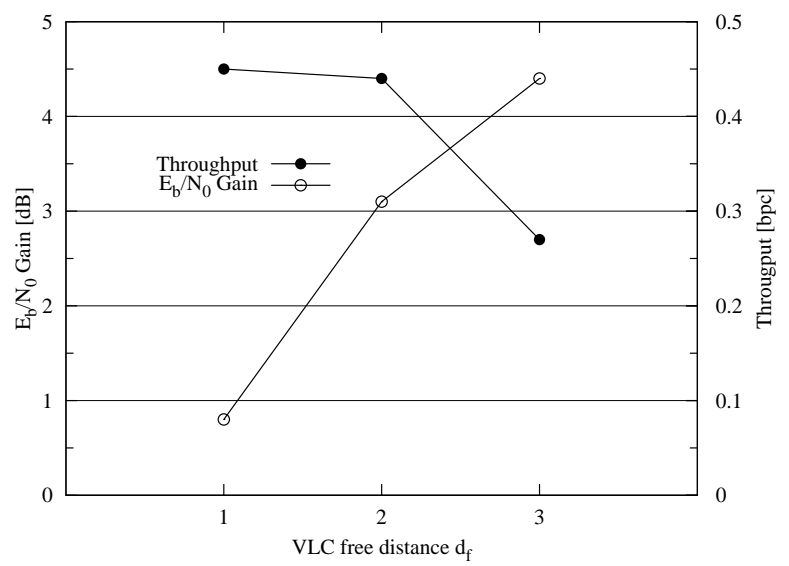

Figure 10.3: Free distance versus $E_{b} / N_{0}$ gain and throughput, where the $E_{b} / N_{0}$ gain is based on the minimum SNR value required for achieving a SER of $10^{-4}$ for transmission over AWGN channels and the scheme using the Huffman code (HUFF) is used as a benchmarker. The system model is described in Fig. ??, where the transmitter is constituted by a VLC encoder and a convolutional encoder, and the receiver is constituted by an APP convolutional decoder as well as an APP VLC decoder, which performs channel decoding and source decoding iteratively.

Given the EXIT characteristics of the constituent modules of a concatenated scheme, we may either predict or explain its convergence behaviour. This is carried out for iterative source/channel decoding for transmission over non-dispersive AWGN channels in Section ?? 
and for transmission over dispersive AWGN channels in Section ??. In the scenario of nondispersive AWGN channels, it was shown in Fig. ?? that the free distance of the source code has to be larger than $d_{f}=2$ in order that the iterative decoding scheme becomes capable of converging to the perfect mutual-information point of $(1,1)$, which implies attaining infinitesimally low SERs. Furthermore, it was shown in Fig. ??-Fig. ?? that given a specific channel code, the system's convergence threshold decreases upon increasing the free distance of the source code, resulting in an improved SER performance. Fig. 10.3 serves as a summary of our main results provided in Section ??. It is worth noting that when the free distance of the VLC code is increased from $d_{f}=1$ to $d_{f}=2$, i.e. when using the code RVLC- 2 instead of the code HUFF or RVLC-1, the system's throughput is only slightly decreased, but a significant $E_{b} / N_{0}$ gain is attained. Further increasing the free distance will continue to increase the attainable $E_{b} / N_{0}$ gain, while incurring a considerable loss of throughput.

In the scenario of dispersive channels, it was shown by both our EXIT chart analysis and our Monte Carlo simulations provided in Section ?? that the redundancy in the source codes is capable of effectively eliminating the ISI imposed by the channel, provided that channel equalisation and source decoding are performed jointly and iteratively. Furthermore, the higher the free distance of the source code, the closer the SER performance approaches the SER bound of non-dispersive AWGN channels.

Additionally, in Section ?? precoding was shown to be an effective way of "modifying" the EXIT characteristic of a channel equaliser. Most importantly, in conjunction with precoding the EXIT function of a channel equaliser becomes capable of reaching the point of $\left(I_{A}=1, I_{E}=1\right)$ as shown in Fig. ??, which is critical for avoiding potential error floors at the receiver's output. It was demonstrated in Fig. ??-Fig. ?? that the choice of the precoder depends on both the EXIT characteristics of the channel equaliser and that of the source decoder so that these two are matched to each other, hence achieving the lowest possible $E_{b} / N_{0}$ convergence threshold.

Fig. 10.4 summarises the main results of Section ??. It can be seen from Fig. 10.4 that the SER performance of both the scheme using RVLC-2 and that using VLEC-3 can be improved, when using appropriate precoders. However, although the precoder of $1+\mathrm{D}^{2}$ is optimal for the scheme using RVLC-2, the precoder of $1+\mathrm{D}$ constitutes a better choice for the scheme using VLEC-3.

Finally, the performance of a three-stage iterative receiver was evaluated in Section ??. The receiver of Fig. ?? consists of a channel equaliser, a channel decoder and a source decoder, where the extrinsic information is exchanged among all the three SISO modules, which hence constitutes a joint source-channel decoding and equalisation scheme. It was shown in Fig. ?? that by exploiting the source redundancy in the iterative decoding process, the system's performance was improved by $2 \mathrm{~dB}$ in terms of the $E_{b} / N_{0}$ values required for achieving the same SER, when compared to the separate source/channel decoding scheme. The convergence behaviour of this scheme was analysed using EXIT charts in Section ?? after we introduced the convergence analysis technique for multi-stage concatenated schemes in Chapter ??. 


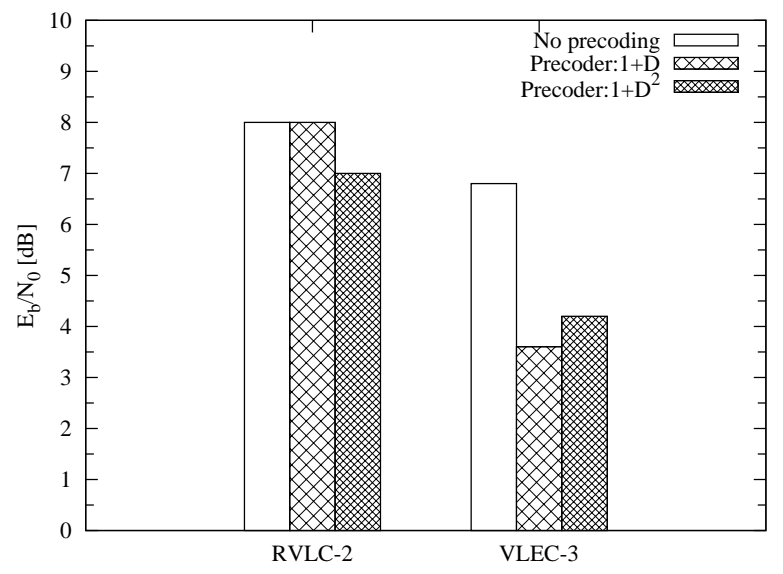

Figure 10.4: The effects of precoding and those of VLC free distances of $d_{f}=2$ for the RVLC- 2 and $d_{f}=3$ for the VLEC-3 schemes on the attainable SER performance, when communicating over dispersive AWGN channels, where the $E_{b} / N_{0}$ value is the minimum SNR value required for achieving a SER of $10^{-4}$. The system model is described in Fig. ?? and Fig. ??, where the transmitter is constituted by a VLC encoder as well as a precoder if precoding is employed, and the receiver is constituted by an APP channel equaliser as well as an APP VLC decoder, which performs channel equalisation and source decoding iteratively.

\subsection{Chapter ??: Three-Stage Serially Concatenated Turbo Equalisation}

Chapter ?? investigated the design of the three-stage serially concatenated turbo MMSE equalisation scheme seen in Fig. ??, which consisted of an inner channel equaliser, a unityrate recursive intermediate channel code and an outer channel code. Firstly, a brief introduction to SISO MMSE equalisation was offered in Section ??, followed by an example of conventional two-stage turbo equalisation in Section ??. The main body of this chapter focused on the optimisation of three-stage turbo equalisation schemes by using EXIT chart analysis.

With the aid of the EXIT modules as proposed in Fig. ?? of Section ??, 3D EXIT chart analysis may be simplified to 2D EXIT analysis as shown in Fig. ??, ?? and ?? of Section ??. It was also shown in Fig. ?? of Section ?? that by employing a unity-rate recursive convolutional code as the intermediate constituent code, the three-stage scheme becomes capable of converging to the perfect mutual information point.

Moreover, the outer constituent code was optimised in Section ?? for achieving the lowest possible $E_{b} / N_{0}$ convergence threshold. Interestingly, it was observed in Fig. ?? that relatively weak codes having short memories resulted in a lower convergence threshold than strong codes having long memories.

Additionally, the activation order of the component decoders was optimised in Section ?? for achieving the convergence at the lowest possible $E_{b} / N_{0}$ value, while maintaining a 
low decoding complexity. It was found in Table ?? that by invoking the outer and intermediate decoder of Fig. ?? more frequently the total number of decoder activations is reduced, resulting in a decreased decoding complexity.

The BER performance of the optimised scheme was evaluated in Section ??, which verified the EXIT chart analysis provided in Section ??. The iterative decoding process was visualised using both 3D and 2D EXIT charts as shown in Fig. ??-?? of Section ??. Furthermore, the effects of different interleaver block lengths were discussed in Fig. ?? of Section ??. Generally, the longer the interleaver length, the closer the simulated performance matches the EXIT chart analysis. It was found in Fig. ?? that an interleaver length on the order of $10^{5}$ bits is sufficiently high for achieving a good match with the decoding trajectory recorded. Fig. 10.5 provides some quantitative results summarised from Section ??. It can be seen from Fig. 10.5 that when the interleaver depth is increased from $L=10^{3}$ bits to $L=10^{4}$ bits, a significant coding gain may be attained. Further increasing the interleaver depth to $L=10^{5}$ bits, however, results in a marginal increase of the coding gain. Naturally, the attainable iteration gain is increased upon increasing the interleaver depth.

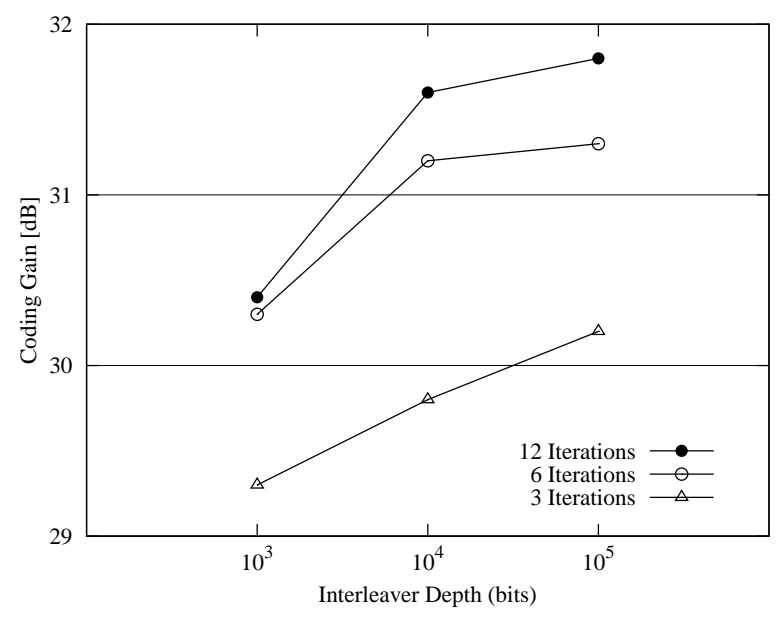

Figure 10.5: Achievable coding gains at a BER of $10^{-4}$ for the three-stage turbo equalisation scheme of Fig. ?? using different interleaver depths. The turbo equalisation scheme is constituted by a $\operatorname{RSC}(2,1,2)$ code as the outer code, a unity-rate $\operatorname{RSC}(1,1,2)$ code as the intermediate code and an inner MMSE equaliser as described in Section ??.

In Section ??, the maximum achievable information rate of the three-stage turbo equalisation scheme of Fig. ?? was analysed. Then an IRCC was invoked as the outer constituent code, whose EXIT function was optimised for matching that of the combined module of the inner channel equaliser and the intermediate channel decoder, so that the EXIT tunnel-area between these two EXIT functions was minimised. The Monte Carlo simulation results provided in Fig. ?? of Section ?? show that the performance of the resultant scheme is only 0.5 $\mathrm{dB}$ away from the channel capacity. 
Finally, the employment of non-unity rate intermediate codes was also considered in Section ??. It was shown in Fig. ?? that as expected, the maximum achievable information rate of such schemes was reduced in comparison to the schemes using unity-rate intermediate codes. By contrast, the $E_{b} / N_{0}$ convergence threshold may be decreased, when only regular convolutional codes are used. A number of optimised serially concatenated codes were obtained and listed in Table ??.

As a summary, Fig. 10.6 compares the distance to capacity for the various MMSE turbo equalisation schemes discussed in Chapter ??.

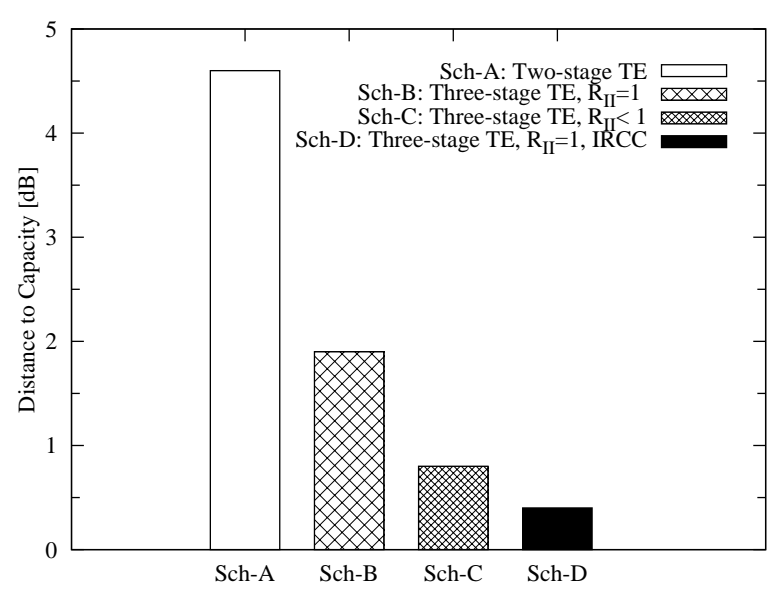

Figure 10.6: Distance to capacity for the various MMSE turbo equalisation schemes of Chapter ??, where the scheme of Sch-A represents the conventional two-stage turbo equalisation scheme of Fig. ??. The schemes of Sch-B, Sch-C and Sch-D denotes the same three-stage turbo equalisation scheme of Fig. ??, but differ in the channel codes used. The scheme of Sch-B employs a unity-rate $\operatorname{RSC}(1,1,2)$ code as the intermediate code and a $\operatorname{RSC}(2,1,2)$ code as the outer code. The scheme of Sch-C uses a SCC of SCC-A2 described in Table ??, which is constituted by a rate-3/4 RSC $(3,4,2)$ code as the intermediate code and a $\operatorname{RSC}(2,3,3)$ code as the outer code. The scheme of Sch-D employs the same unity-rate RSC $(1,1,2)$ code used in the scheme of Sch-B as the intermediate code, while using the IRCC described in Section ?? as the outer code.

In Part II of this book, we have introduced the novel concept of Irregular Variable Length Coding (IrVLC) and investigated its applications, characteristics and performance in the context of wireless telecommunications. As discussed throughout Part II of the book, IrVLCs encode various components of the source signal with different sets of binary codewords, having a range of appropriately selected lengths. Three particular applications of IrVLCs were investigated in this volume, namely joint source and channel coding, EXtrinsic Information Transfer (EXIT) chart matching and Unequal Error Protection (UEP). These are detailed in the following sections, together with a discussion of our future work. 


\subsection{Chapter 6: Joint source and channel coding}

In Chapter 6 we exemplified the application of IrVLCs for the joint source and channel coding of video information. This application was motivated by the observation that Shannon's source and channel coding separation theorem [24] is invalid in the context of practical video transmission. While source and channel coding can be performed in isolation without imposing any performance loss, if the assumptions discussed in Section 6.1 apply, these conditions are not fulfilled in the case of practical video transmission. We therefore proposed the novel joint source and channel coding scheme of Section 6.2, which employs both Variable Dimension Vector Quantisation (VDVQ) [194] as a special case of Vector Quantisation (VQ) [80] and the Reversible Variable Length Coding (RVLC) [99] class of Variable Length Codes (VLCs).

Here, the employment of VDVQ tiles having a range of dimensions facilitates the efficient representation of both large areas of the video frame that have a low luminance-variance and small areas of high variance, as exemplified in Figure 6.5. Additionally, the employment of RVLC codewords having various lengths facilitates the representation of more frequently occurring VDVQ tiles with the aid of shorter codewords, giving a reduced average codeword length and providing source coding. Furthermore, channel coding is provided by the redundancy that is inherent in the RVLC codewords [99], facilitating an error correction capability during RVLC decoding. The VDVQ/RVLC video codec advocated therefore employs a joint source and channel coding philosophy.

In Section 6.3.3 we imposed a number of constraints governing the allocation of the VDVQ tiles and RVLC codebooks in order to represent the various components of the video source frame. More specifically, these code constraints enforced the legitimate tessellation of the VDVQ tiles having a range of dimensions and ensured that the various fractions of the source video frame were encoded using the same number of bits. Since the set of RVLC codewords that can be employed during video encoding varies depending on which component of the source video frame is being encoded, the VDVQ/RVLC video codec can be said to employ IrVLCs.

In the VDVQ/RVLC video codec, the complete set of the above-mentioned code constraints was described by the novel trellis structure of Section 6.3.4, which is reminiscent of a symbol-based VLC trellis [2]. Hence, the employment of this trellis structure facilitated the consideration of all legitimate transmission frame permutations. This fact was exploited in order to perform novel Minimum Mean Squared Error (MMSE) VDVQ/RVLC encoding using a variation of the Viterbi algorithm [3], as described in Section 6.4.

Additionally, the employment of the trellis structure during VDVQ/RVLC decoding was shown to guarantee the recovery of legitimate - although not necessarily error-free - video information in Section 6.5. This ensured that useful video information was never discarded, unlike in the conventional video decoders of [181,182], where a single transmission error may render an entire video frame invalid. A novel modification of the Bahl-Cocke-Jelinek-Raviv (BCJR) algorithm [4] was employed during A Posteriori Probability (APP) Soft-In Soft-Out (SISO) VDVQ/RVLC decoding in order to facilitate the iterative exchange [132] of extrinsic information with a serially concatenated APP SISO Trellis Coded Modulation (TCM) [129] decoder, as well as to facilitate the soft MMSE reconstruction of the video sequence. Since the VDVQ/RVLC trellis structure describes the complete set of VDVQ/RVLC-induced code 
constraints, all of the associated redundancy was beneficially exploited with the aid of the modified BCJR algorithm.

In Section 6.6 the serially concatenated and iteratively decoded VDVQ/RVLC-TCM scheme of Section 6.2 was shown to outperform two suitably designed separate source- and channelcoding benchmarkers. This was attributed to the benefits of the VDVQ/RVLC codec described above, which were realised owing to the joint source and channel coding philosophy adopted. Indeed, Figure 6.12 shows that the VDVQ/RVLC-TCM scheme was capable of achieving subjectively pleasing video reconstructions having a Peak Signal to Noise Ratio (PSNR) of $29.5 \mathrm{~dB}$ at a channel Signal to Noise Ratio (SNR) that is $1.1 \mathrm{~dB}$ lower than that of the VQ based benchmarker [181] and $1.6 \mathrm{~dB}$ lower than that of the MPEG-4 [68] based benchmarker [182].

\subsection{Chapters 7 - 9: EXIT chart matching}

In Chapters 7-9 we considered the application of IrVLCs for EXIT chart matching. This was motivated by the fact that an open EXIT chart tunnel was only created for the VDVQ/RVLCTCM scheme of Section 6.2, if the Rayleigh fading channel SNR was in excess of a threshold that was $1.29 \mathrm{~dB}$ higher than the channel's SNR capacity bound, as shown in Figure 10.1. Note that as described in Section ??, an infinitesimally low probability of decoding error can only be achieved, if the EXIT chart tunnel is open and if the iterative decoding trajectory approaches the inner and outer EXIT functions sufficiently closely to facilitate iterative decoding convergence to the $(1,1)$ point of the EXIT chart. Hence, operation closer than $1.29 \mathrm{~dB}$ from the channel's capacity bound was prevented for the VDVQ/RVLC-TCM scheme, as shown in Figure 6.12. Note that similar discrepancies of $1 \mathrm{~dB}$ were obtained for the SBVLC-TCM and BBVLC-TCM schemes of Section 7.3.2, as shown in Figure 10.1. Like the VDVQ/RVLC-TCM scheme of Section 6.2, the SBVLC-TCM and BBVLC-TCM schemes employed the serial concatenation and iterative decoding of a VLC-based outer codec with a TCM inner codec and were not designed using EXIT chart matching. Furthermore, Figure 10.1 shows that a similar discrepancy of $1.4 \mathrm{~dB}$ between the threshold $E_{b} / N_{0}$ value and the channel's attainable capacity bound was obtained for the VLC-URC scheme of Section 9.5.4, which employs Unity Rate Coding (URC) for the inner codec instead of TCM. Instead of the capacity bound, the channel's attainable capacity bound is considered in this case, since it is this that imposes the fundamental limit on the VLC-URC scheme's operation, as described in Section 9.5.3. This is justified, since we will propose a solution to the associated effective throughput loss in Section 10.12, outlining our future work. The corresponding EXIT chart obtained for the VLC-URC scheme of Section 9.5.4 was provided in Figure 9.10, together with those of the other schemes introduced in Section 9.5.4, which are repeated for convenience in Figure 10.7.

In Section 6.6, we observed that open EXIT chart tunnels could have been created for channel SNRs that are closer to the channel's capacity bound, if the inverted VDVQ/RVLC EXIT function of Figure 6.11 offered a better match with the TCM scheme's EXIT function. More specifically, this would have enabled the EXIT chart tunnel to remain open and be further narrowed as the channel SNR was reduced towards the channel's capacity bound. The described observation of Section 6.6 may be explained by the area property of EXIT charts [19], which states that the EXIT chart area enclosed by the threshold EXIT chart 
Table 10.1: Iterative decoding performance and complexity of the various schemes considered in Chapters $6-9$. $\dagger$ The IrVLC comprises the component VLEC codebooks $\left\{\text { VLEC }^{n}\right\}_{n=12}^{22}$ of Table 8.6, which were designed using the GA of Section 8.3. $\star$ The channel's attainable $E_{b} / N_{0}$ capacity bound is employed. 


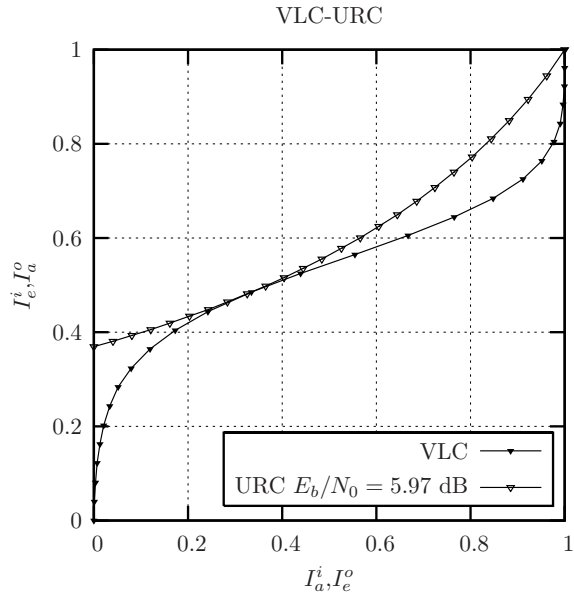

(a)

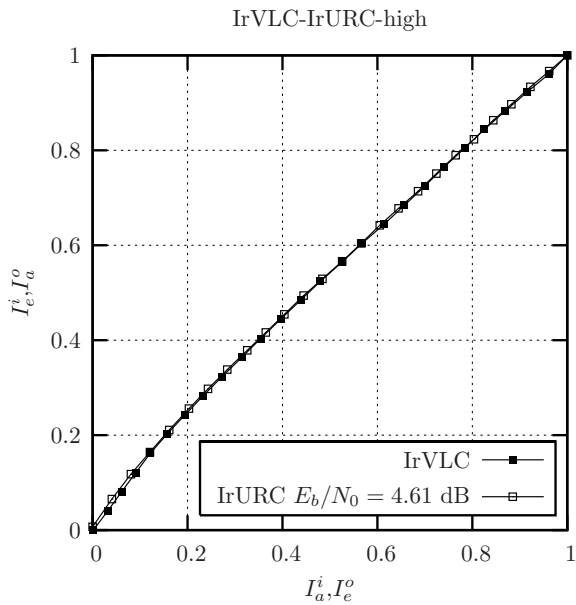

(c)

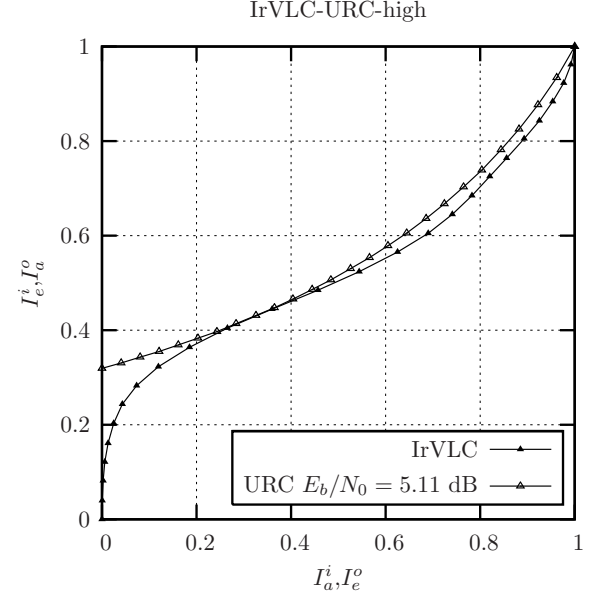

(b)

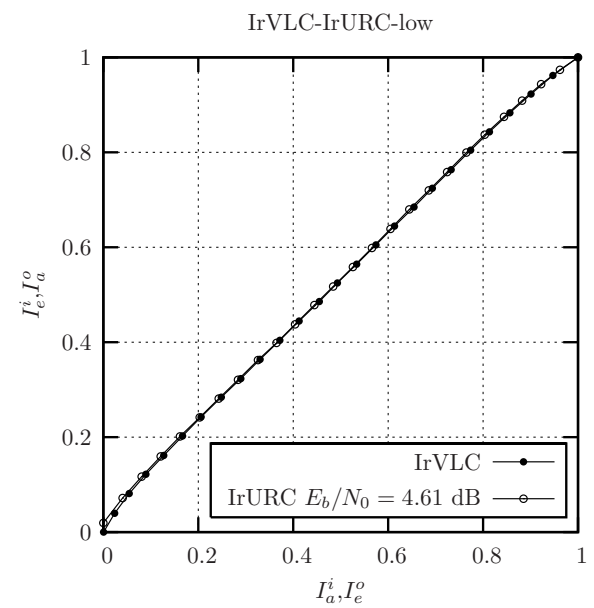

(d)

Figure 10.7: EXIT charts for the schemes of Section 9.5.4. (d) VLC-URC arrangement. (c) IrVLC-URC-high arrangement. (a) IrVLC-IrURC-high arrangement. (b) IrVLC-IrURC-low arrangement. The inner EXIT functions are provided for the threshold channel $E_{b} / N_{0}$ values, as specified in Table 9.1. 
tunnel is commensurate with the discrepancy between the channel's capacity bound and the threshold SNR.

Hence, in Section 7.3.2 we demonstrated that the inverted EXIT function of an outer IrVLC codec can be shaped to match with an inner EXIT function. Here, the IrVLC scheme generated particular fractions of the IrVLC-encoded transmission frame using different component VLC codebooks of either the RVLC or the Variable Length Error Correction (VLEC) [89] class. We showed that the inverted EXIT function of the corresponding APP SISO IrVLC decoder depends on the specifically chosen fractions of the IrVLC-encoded transmission frame that are generated by each component VLC codebook. More explicitly, the inverted IrVLC EXIT function may be obtained using the equation of (7.4), which employs the described fractions as weights during the averaging of the component VLC codebooks' inverted EXIT functions.

Section 7.3.2 showed that the EXIT chart matching algorithm of [10] may be employed to design specific parameterisations of the SBIrVLC-TCM and BBIrVLC-TCM schemes detailed in Section 7.2. Here, the algorithm of [10] was employed to shape the inverted IrVLC EXIT functions to match the EXIT function of the serially concatenated TCM codec. This facilitated the creation of open EXIT chart tunnels at channel $E_{b} / N_{0}$ values in excess of a threshold that is $0.5 \mathrm{~dB}$ from the channel's capacity bound, as shown in Table 10.1. This is equal to the $0.5 \mathrm{~dB}$ discrepancy shown in Table 10.1 that was obtained, when matching the inverted EXIT function of an Irregular Convolutional Code (IrCC) [175] to the TCM EXIT function during the parameterisation of the Huffman-IrCC-TCM scheme of Section 7.4.1.

Furthermore, Table 10.1 shows that the open EXIT chart tunnel of Figure 10.7b was achieved at a similar $E_{b} / N_{0}$ discrepancy of $0.54 \mathrm{~dB}$ from the channel's attainable capacity bound for the IrVLC-URC-high arrangement detailed Section 9.5.4. Note that this scheme employed a serial concatenation of an IrVLC outer codec and a URC inner codec. A URC inner codec was also employed by the IrVLC-URC scheme of Section 8.4. Discrepancies of $0.42 \mathrm{~dB}$ and $0.7 \mathrm{~dB}$ are shown in Table 10.1 for parameterisations of this scheme that employed IrVLC coding rates of 0.55 and 0.85 , respectively. This suggests that an improved EXIT chart matching was achieved when employing lower IrVLC coding rates, resulting in open EXIT chart tunnels at channel $E_{b} / N_{0}$ values that are closer to the channel's capacity bound, as shown in Figures 8.12 and 9.9.

Owing to the aforementioned benefits of EXIT chart matching, the observed discrepancies in the range of $0.42 \mathrm{~dB}-0.7 \mathrm{~dB}$ are lower than those obtained when EXIT chart matching was not employed, which are in the range of $1 \mathrm{~dB}-1.4 \mathrm{~dB}$, as described above.

\subsection{Chapter 8: GA-aided Design of Irregular VLC Components}

Chapter 8 showed that our ability to perform EXIT chart matching and to achieve an open EXIT chart tunnel at $E_{b} / N_{0}$ values that are close to the channel's capacity bound is commensurate with the degree of diverse shapes exhibited by the inverted EXIT functions of the component VLC codebook suite. For this reason, the conventional irregular coding design process strives for obtaining a component VLC codebook suite having a wide variety of inverted EXIT functions, as shown in Figure 10.8. The component VLEC codebooks employed by the IrVLC schemes of Chapters 7 and 8 were designed using Algorithm E of Section ??. As discussed in Section 8.1, this algorithm attempts to design VLEC codebooks having maximal coding rates that satisfy particular specified distance criteria. However, this algorithm 
does not facilitate the direct control or prediction of the inverted EXIT function shapes that correspond to the designed VLEC codebooks. Hence, in the conventional irregular coding design process depicted in Figure 10.8, a significant amount of trial-and-error based human interaction is required. This involves the design of a high number of candidate component VLEC codebooks, the characterisation of their inverted EXIT functions and the selection of a suite having a wide variety of inverted EXIT functions, as exemplified in Chapter 7.

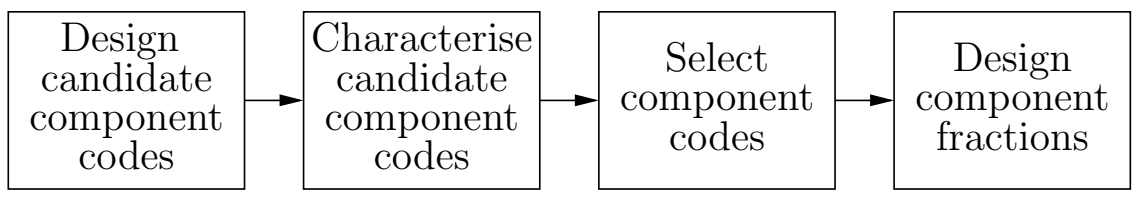

Figure 10.8: Conventional irregular coding design process.

The trial-and-error efforts required to design a suite of IrVLC component codebooks using Algorithm E of Section ?? motivated the design of a novel Genetic Algorithm (GA) for generating the VLEC codebooks of Section 8.3. Unlike Algorithm E of Section ??, this GA was shown to facilitate the direct control and prediction of the inverted EXIT function shapes that result for the designed VLEC codebooks, eliminating the trial-and-error efforts in the irregular coding design process. While maintaining desirable VLEC-encoded bit entropies and IrVLC decoding complexities, the GA of Section 8.3 seeks VLEC codebooks having arbitrary coding rates and Real-Valued Free Distance Metrics (RV-FDMs).

This novel RV-FDM was proposed in Section 8.2 as an alternative to the Integer-Valued Free Distance (IV-FD) lower bound of [89] for the characterisation of a VLEC codebook's error correction capability. Like the IV-FD lower bound of [89], the RV-FDM considers the minimum number of differing bits in any pair of equal-length legitimate VLEC-encoded bit sequences, characterising the probability of occurrence for the most likely undetectable transmission error scenario, as described in Section 8.1. However, unlike the IV-FD lower bound, the RV-FDM of Section 8.2 also considers how susceptible the VLEC-encoded bits are to this transmission error scenario. As a result, the RV-FDM exists within the real domain, allowing the comparison of the error correction capabilities of two VLEC codebooks having equal IV-FD lower bounds. This facilitates its employment within the objective function of the novel GA proposed in Section 8.3.

In Section 8.2, we showed that a VLEC codebook's RV-FDM affects the number of inflection points appearing in the corresponding inverted EXIT function. More specifically, we showed that high RV-FDMs are associated with ' $S$ '-shaped inverted EXIT functions having up to two points of inflection, whilst low RV-FDMs result in inverted EXIT functions having no more than one point of inflection. Furthermore, we showed that the inverted EXIT function of a VLEC codebook will reach the top right hand corner of the EXIT chart if its RV-FDM is at least equal to two [161]. These findings complement the property [19] that the area below an inverted VLEC EXIT function equals the corresponding coding rate. Therefore, since the inverted VLEC EXIT function shape of a VLEC codebook depends on both its coding rate and RV-FDM, the GA of Section 8.3 facilitates the direct control and prediction of the inverted EXIT function shapes that result for the designed VLEC codebooks. 
The employment of both the novel GA of Section 8.3 and Algorithm E of Section ?? to design suites of IrVLC component codebooks was investigated in Section 8.5. The suite of component VLEC codebooks designed in Section 8.5.1 by our novel GA had the wide variety of inverted EXIT functions shown in Figure 8.8. This was obtained by seeking component VLEC codebooks having a wide variety of coding rates and RV-FDMs. In some cases, high RV-FDMs were sought, resulting in ' $S$ '-shaped inverted EXIT functions having up to two points of inflection, whilst low RV-FDMs were sought for the remaining component VLEC codebooks, which were associated with inverted EXIT functions having no more than one point of inflection. Here, we found that more extreme RV-FDMs could be obtained for VLEC codebooks having lower coding rates. This may be explained by the higher degree of design freedom that is facilitated for lower coding rates owing to the longer codewords that this implies.

Similarly to when the novel GA of Section 8.3 was employed to design component VLEC codebooks, trial-and-error was not employed when Algorithm E of Section ?? was used, facilitating a fair comparison. Instead, a different IV-FD lower bound was sought for each component VLEC codebook designed using Algorithm E of Section ??. However, the resultant component VLEC codebooks were found to have relatively high RV-FDMs and only a limited variety of coding rates, resulting in the limited variety of ' $S$ '-shaped inverted EXIT functions shown in Figure 8.7.

Owing to its employment of a wider variety of coding rates and as a benefit of its both high as well as low RV-FDMs, the suite of component VLEC codebooks designed by our novel GA in Section 8.5.1 was found to be more suitable for use in EXIT chart matching than that designed using Algorithm E of Section ??. More specifically, open EXIT chart tunnels could be created for the IrVLC-URC scheme of Section 8.4 at channel $E_{b} / N_{0}$ values within $1 \mathrm{~dB}$ of the Rayleigh fading channel's capacity bound for a wide range of effective throughputs, when employing the suite of component VLEC codebooks generated using our GA, as shown in Figure 8.12. By contrast, open EXIT chart tunnels could only be achieved when employing the suite designed by Algorithm E of Section ?? for a limited range of effective throughputs and within a significantly higher margin of $4.4 \mathrm{~dB}$ from the $E_{b} / N_{0}$ capacity bound. This confirmed the observation that our ability to perform EXIT chart matching depends on how much variety is exhibited within the inverted EXIT functions of the suite of component VLEC codebooks.

However, regardless of the component VLEC codebook suite employed, we observed in Section 8.5.4 that the inverted IrVLC EXIT function can only be matched to the EXIT functions of a regular inner codec with limited accuracy. This is because inverted outer EXIT functions are constrained to starting from the $(0,0)$ point of the EXIT chart, while the inner EXIT functions typically emerge from a relatively high point along the $I_{e}^{i}$ axis of the EXIT chart, as described in Section ??. As a result, we cannot create an arbitrarily narrow open EXIT chart tunnel. Instead, a lower bound is imposed upon the enclosed EXIT chart area and, hence, upon the discrepancy between the threshold $E_{b} / N_{0}$ value and the channel's capacity bound, owing to the area property of EXIT charts [19]. 


\subsection{Chapter 9: Joint EXIT Chart Matching of IRVLCs and IRURCs}

The above-mentioned findings motivated the introduction of novel Irregular Unity Rate Codes (IrURC) in Chapter 9, which encode different fractions of the transmission frame using different component URCs, having various EXIT functions. In analogy to those of IrVLCs and IrCCs, IrURC EXIT functions may be shaped by specifically selecting the fraction of the transmission frame that is encoded by each component URC. In this way, the IrURC EXIT function may be shaped to emerge from a point on the EXIT chart's $I_{e}^{i}$ axis that is closer to the inverted outer EXIT function's starting point of $(0,0)$.

The serial concatenation and iterative decoding of an IrVLC outer codec with an IrURC inner codec was demonstrated in Section 9.4. Here, the IrVLC's suite of component VLEC codebooks was designed using the GA of Section 8.3 in order to generate the required diversity of inverted EXIT function shapes shown in Figure 9.5 and repeated for convenience in Figure 10.9. By contrast, the EXIT functions shown in Figure 9.7 and repeated for convenience in Figure 10.10 were obtained by selecting the IrURC's suite of component URCs from a large number of candidates, as described in Section 9.5.2. In Section 9.3, we proposed a novel method for jointly matching the EXIT functions of the two serially concatenated irregular codecs. This method iteratively applies the EXIT chart matching algorithm of [10] to alternately match the outer EXIT function to the inner and vice versa, simultaneously seeking the highest coding rate that offers an open EXIT chart tunnel. Note however, that the novel modification of Section 9.2 was required in order to allow the EXIT chart matching of the IrURC EXIT function, since all component URCs have the same unity coding rate. The joint EXIT chart matching algorithm of Section 9.3 was shown to be able to exploit the increased degree of design freedom that is afforded by employing two irregular codecs in order to create an EXIT chart tunnel that is narrow at all points along its length. This facilitated the creation of the marginally open EXIT chart tunnels shown in Figures 10.7c and 10.7d for the IrVLC-IrURC-high and IrVLC-IrURC-low arrangements of Section 9.5.4, respectively. Owing to the area property of EXIT charts, these were obtained at $E_{b} / N_{0}$ values that were just $0.04 \mathrm{~dB}$ from the channel's attainable capacity bound, as shown in Figure 10.1.

Note that an open EXIT chart tunnel implies that iterative decoding convergence to an infinitesimally low probability of error can be achieved, provided that the iterative decoding trajectory approaches the inner and outer EXIT functions sufficiently closely, as described in Section ??. However, throughout this monograph we found that high quality reconstructions could not be achieved at the threshold $E_{b} / N_{0}$ values, where the EXIT chart tunnels open. This is owed to the BCJR algorithm's assumption [4] that all correlation within the LLR frames exchanged by the APP SISO decoders is successfully mitigated by the intermediary interleavers. If this is not the case, the iterative decoding trajectory will not match perfectly with the inner and outer EXIT functions and the tunnel must be further widened before the trajectory can reach the top right hand corner of the EXIT chart, which is associated with an infinitesimally low probability of error, as described in Section ??. Since the interleaver's ability to mitigate the correlation is proportional to its length, longer interleavers can be expected to yield lower discrepancies between the $E_{b} / N_{0}$ value at which the EXIT chart tunnel opens and that at which it is sufficiently widened to facilitate a high reconstruction quality. Indeed, this relationship may be observed in Figure 10.11, which provides a scatter 


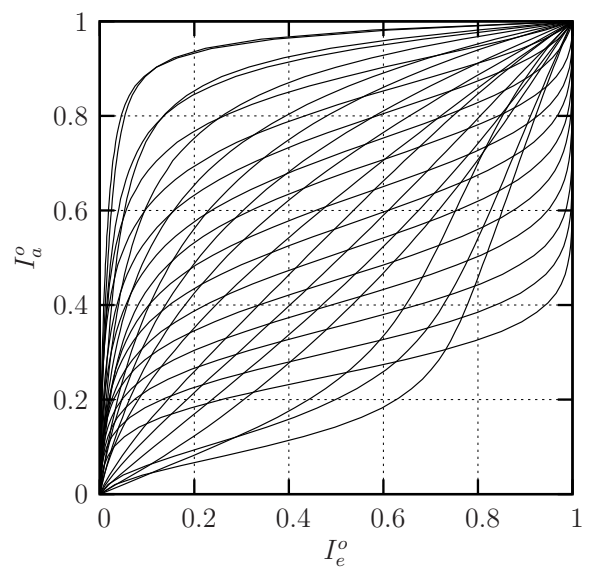

Figure 10.9: Inverted EXIT functions for the component VLEC codebooks employed by the IrVLC-IrURC-high and IrVLC-IrURC-low arrangements of Section 9.5.4.

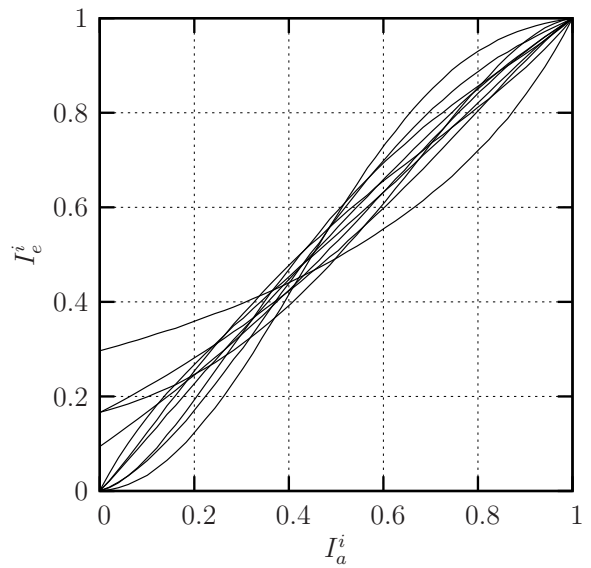

Figure 10.10: EXIT functions corresponding to a Gray-coded 16QAM-modulated Rayleigh fading channel SNR of $8 \mathrm{~dB}$ for the component URC codes employed by the IrVLC-IrURChigh and IrVLC-IrURC-low arrangements of Section 9.5.4. 
plot of the discrepancies and interleaver lengths given in Table 10.1, as will be detailed in our forthcoming discussions.

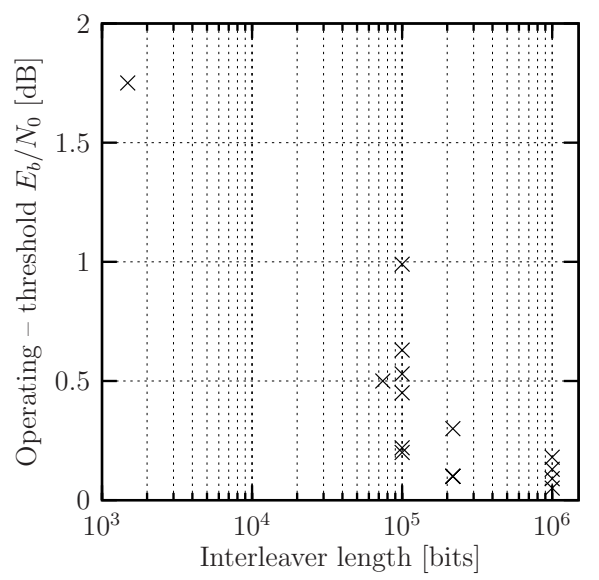

Figure 10.11: Scatter plot of the interleaver lengths provided in Table 10.1 and the corresponding discrepancies between the $E_{b} / N_{0}$ value at which EXIT chart tunnel opens and that at which it is sufficiently widened to facilitate a high reconstruction quality.

In Section 6.6, we characterised the discrepancy between the threshold $E_{b} / N_{0}$ value at which an open EXIT chart tunnel could be created for the VDVQ/RVLC-TCM video transmission scheme and the operating $E_{b} / N_{0}$ value at which it could achieve a high quality reconstruction having a Peak Signal to Noise Ratio (PSNR) of $29.5 \mathrm{~dB}$. This discrepancy was found to be $1.75 \mathrm{~dB}$, when the interleaver length was equal to that of a single encoded video frame, namely 1485 bits, as shown in Table 10.1. By contrast, when 50 encoded video frames were concatenated to give an interleaver length of 74250 bits, the discrepancy was reduced to just $0.5 \mathrm{~dB}$, facilitating operation at $1.79 \mathrm{~dB}$ from the channel's $E_{b} / N_{0}$ capacity bound. However in Section 6.6, this scheme was shown to incur a $5 \mathrm{~s}$ latency, since the video frame rate was $10 \mathrm{fps}$ and because all 50 frames must be received before they can be deinterleaved.

The discrepancy between the threshold $E_{b} / N_{0}$ value at which an open EXIT chart tunnel could be created for the arrangements of Section 9.5.4 and the operating $E_{b} / N_{0}$ value at which they could achieve a BER of $10^{-5}$ was characterised in Section 9.6. When a 100000 bit interleaver was employed, the discrepancies for the VLC-URC and IrVLC-URC-high arrangements were found to be $0.2 \mathrm{~dB}$ and $0.22 \mathrm{~dB}$, respectively, as shown in Table 10.1. However, these discrepancies were reduced to $0.05 \mathrm{~dB}$ and $0.09 \mathrm{~dB}$, respectively, when we employed a longer interleaver, having a length of 1000000 bits. Larger discrepancies were observed for the IrVLC-IrURC-high and IrVLC-IrURC-low arrangements, owing to their narrow EXIT chart tunnels, as discussed in Section 9.6. These were $0.53 \mathrm{~dB}$ and $0.67 \mathrm{~dB}$, respectively, when the 100000 -bit interleaver was employed, as compared to $0.18 \mathrm{~dB}$ and $0.13 \mathrm{~dB}$, respectively, when the 1000000 -bit interleaver was employed. Note that the IrVLCIrURC-low arrangement using the 1000 000-bit interleaver could achieve a BER of less than $10^{-5}$ for $E_{b} / N_{0}$ in excess of a limit that was just $0.17 \mathrm{~dB}$ from the channel's attainable capacity bound, as shown in Table 10.1 . This is comparable to the $0.13 \mathrm{~dB}$ discrepancy 
demonstrated for Irregular Low Density Parity Check (IrLDPC) codes $[58,166]$ and superior to the $0.25 \mathrm{~dB}$ discrepancy found for irregular turbo codes [59].

In Section 7.4, we showed that the SBVLC-, BBVLC-, SBIrVLC- and BBIrVLC-TCM schemes using a 217500 -bit interleaver could achieve a high quality source sample reconstruction SNR of $20 \mathrm{~dB}$ for $E_{b} / N_{0}$ values in excess of a limit that was $0.1 \mathrm{~dB}$ from the threshold at which an open EXIT chart tunnel was created, as shown in Table 10.1. However, in the case of the Huffman-IrCC-TCM scheme of Section 7.4.1, the corresponding discrepancy was equal to the higher value of $0.3 \mathrm{~dB}$. This was explained in Section 7.4.3 by the relatively high sensitivity of the APP SISO IrCC decoder to any residual correlation within the iteratively exchanged LLRs, that was insufficiently mitigated by the 217500 -bit interleaver. This resulted in the poor correlation between the iterative decoding trajectory and the inverted IrCC EXIT function. More specifically, we concluded that an APP SISO IrCC decoder's sensitivity to this correlation increased if its coding rate is reduced or if, in particular, we increase its coding memory. Hence, the high sensitivity of the Huffman-IrCC-TCM scheme's APP SISO IrCC decoder was attributed to its relatively high coding memory of 4.

Section 7.4.3 also concluded that an APP SISO VLC decoder's sensitivity to the aforementioned correlation depends only on its coding rate. Indeed, in Section 8.6, the APP SISO IrVLC decoder's sensitivity to this extrinsic information correlation was found to increase as the IrVLC coding rate was reduced. As shown in Table 10.1, the IrVLC-URC scheme of Section 8.4 using a 100000 -bit interleaver and an IrVLC coding rate of 0.85 could achieve a BER of less than $10^{-5}$ for $E_{b} / N_{0}$ values in excess of a limit that was $0.45 \mathrm{~dB}$ from the threshold at which an open EXIT chart tunnel was created. However, this discrepancy grew to $0.99 \mathrm{~dB}$ when the IrVLC coding rate was reduced to 0.55 , as shown in Table 10.1 .

Throughout this book we have considered receivers in which soft information is iteratively exchanged between APP SISO decoders and in which a final hard decision is made by a MAP sequence estimator. These components of the receiver apply the BCJR algorithm [4] and the Viterbi algorithm [3] to suitably designed trellises [52,90]. These require only Add, Compare and Select (ACS) operations if all calculations are performed in the logarithmic probability domain and if a lookup table is employed for correcting the Jacobian approximation [197]. Since each individual ACS operation requires the same resources in a systolicarray based chip, the number of ACS operations performed by a receiver may be employed to characterise the complexity/area/speed trade-off required for its implementation.

In Section 7.4, we introduced the novel plot of Figure 7.12 for characterising the iterative decoding complexity of a receiver. This plot provides the average number of ACS operations required per source symbol to achieve particular reconstruction qualities as a function of the channel's $E_{b} / N_{0}$ value. This plot, as well as those of Figures 7.13, 8.17, 8.18 and 9.12, showed that particular reconstruction qualities can be achieved with lower complexities as the channel's $E_{b} / N_{0}$ value is increased. This may be explained by the associated widening of the open EXIT chart tunnel, requiring fewer decoding iterations for the iterative decoding trajectory to reach the particular point on the EXIT chart that is associated with the reconstruction quality considered.

Additionally, Figures 7.12 and 7.13 showed that lower complexities may be maintained, provided that lower reconstruction qualities can be tolerated, since less decoding iterations are required for the iterative decoding trajectory to reach the particular point on the EXIT chart that is associated with a lower reconstruction quality. However, Section 7.4.5 observed 
that in the approach of iterative decoding convergence, large reconstruction quality gains are obtained for relatively small amounts of additional computational complexity. We concluded that if the channel $E_{b} / N_{0}$ value is sufficiently high to create an open EXIT chart tunnel, then we can typically justify the computational complexity required for the iterative decoding trajectory to reach the $(1,1)$ point of the EXIT chart, owing to the infinitesimally low probability of error that results.

In Sections 7.4.5, 8.6 and 9.5.4 we showed that outer APP SISO decoding and MAP sequence estimation are typically associated with significantly higher computational complexities than inner APP SISO decoding. In the most extreme case considered in this volume, the outer decoders of the SBVLC- and SBIrVLC-TCM schemes of Section 7.2 accounted for about $97 \%$ of the ACS operations employed per source sample. By contrast, the outer decoders of the Huffman-IrCC-TCM scheme of Section 7.4.1 were responsible for about $60 \%$ of the iterative decoding complexity, in the most balanced case considered.

In Table 10.1, we provide the average number of ACS operations required per source symbol to achieve high quality reconstructions at an $E_{b} / N_{0}$ value that is $2 \mathrm{~dB}$ from the channel's capacity bound for each of the schemes considered in Sections 7.2 and 8.5.4. Additionally, for the schemes of Section 9.6, the complexity at an $E_{b} / N_{0}$ value that is $2 \mathrm{~dB}$ from the channel's attainable capacity bound is provided in Table 10.1. Furthermore, Figure 10.12 plots the complexities of the aforementioned schemes for a range of $E_{b} / N_{0}$ discrepancies from the capacity bounds. While the complexities shown in Table 10.1 and Figure 10.12 for the schemes of Section 7.2 are associated with obtaining a high-quality source sample reconstruction SNR of $20 \mathrm{~dB}$, those provided for the schemes of Sections 8.5.4 and 9.6 are associated with achieving a BER of $10^{-5}$. The comparison of the described complexities is fair, since each of the schemes considered in Chapters $7-9$ facilitates the transmission of 16-ary source symbols over an uncorrelated narrowband Rayleigh fading channel. Furthermore, in all cases, the source symbols have the probabilities of occurrence that result from the Lloyd-Max quantisation $[74,75]$ of Gaussian distributed source samples, as described in Section 7.2.1.

Note that Figure 10.12 illustrates the discrepancies between the channel's appropriate capacity bounds and the $E_{b} / N_{0}$ values above which the schemes considered in Chapters 7 - 9 may achieve high quality reconstructions, confirming the discrepancies provided in Table 10.1. Furthermore, at high discrepancies from the channel's $E_{b} / N_{0}$ capacity bounds, Figure 10.12 shows that similar iterative decoding complexities may be observed for the BBVLC-, BBIrVLC- and Huffman-IrCC-TCM schemes of Section 7.2 as well as for each scheme introduced in Sections 8.5.4 and 9.6. Indeed, the corresponding ACS counts provided in Table 10.1 can be seen to have similar values in the range of $\left[3.1 \times 10^{4}, 8.6 \times 10^{4}\right]$. This similarity may be explained because all of these schemes employ bit-based trellises $[52,90]$ as the basis of their outer APP SISO decoders and MAP sequence estimators. By contrast, the SBVLC- and SBIrVLC-TCM schemes of Section 7.2 employed the symbol-based VLC trellis of [2] as the basis of their APP SISO decoders. For these schemes, Table 10.1 provides ACS operation counts of $3.5 \times 10^{5}$ and $1.2 \times 10^{6}$, respectively, which are significantly higher than those provided for the schemes employing bit-based trellises, as illustrated in Figure 10.12. This increased complexity may be attributed to the number of trellis transitions that are employed in symbol-based VLC trellises, which is typically significantly higher than the number employed in their bit-based equivalents, as described in Section ??. 

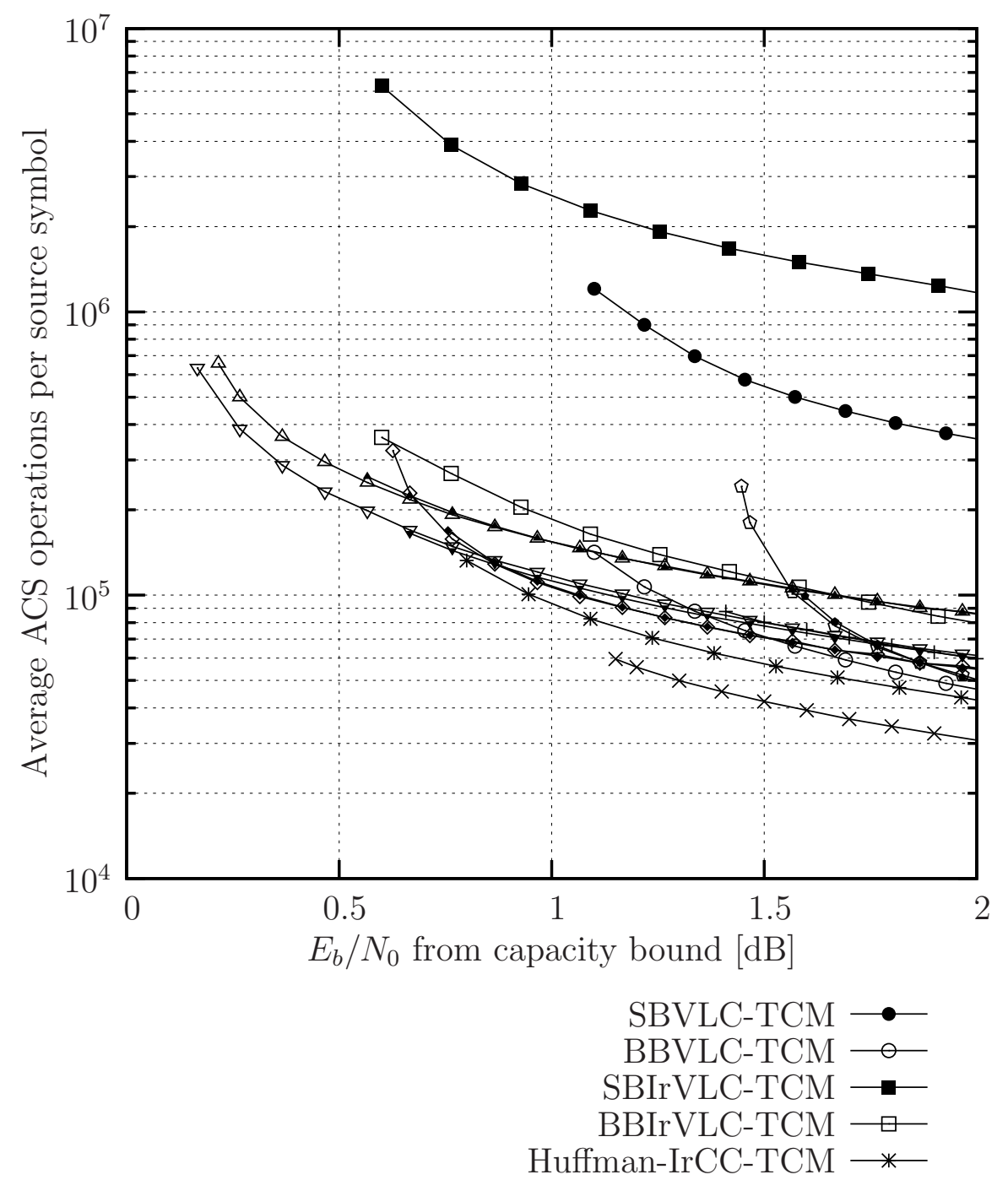

IrVLC-URC 0.55-rate outer codec — IrVLC-URC 0.85-rate outer codec $\longrightarrow$ VLC-URC 100 000-bit interleaver —— VLC-URC 1000 000-bit interleaver —IrVLC-URC-high 100 000-bit interleaver —— IrVLC-URC-high 1000 000-bit interleaver $\multimap$ IrVLC-IrURC-high 100 000-bit interleaver _. IrVLC-IrURC-high 1000 000-bit interleaver $\_\_$ IrVLC-IrURC-low 100 000-bit interleaver $\longrightarrow$ IrVLC-IrURC-low 1000 000-bit interleaver $\longrightarrow$

Figure 10.12: Average number of ACS operations per source symbol required to achieve high quality reconstructions at a range of $E_{b} / N_{0}$ discrepancies from the appropriate capacity bounds for the schemes of Chapters $7-9$. 
The computational complexities provided in Figure 10.12 for the IrVLC-URC arrangement of Section 8.5.4 having an IrVLC coding rate of 0.85 can be seen to be lower than that associated with an IrVLC coding rate of 0.55 . This may be explained by the higher number of bit-based trellis transitions that are required to represent the longer codewords of the 0.55-rate IrVLC, as discussed in Section 8.6. For this reason, we can expect lower computational complexities to be associated with VLCs and IrVLCs having higher coding rates in general. This is confirmed by the average numbers of ACS operations performed per source symbol that are provided for VLEC codebooks having various coding rates in Figures 8.7 - 8.10 and 9.5. These figures additionally show that VLEC codebooks having relatively low RV-FDMs are also associated with low computational complexities. This was exploited during the design of the IrVLC-IrURC-low arrangement of Section 9.5.4. More specifically, the novel modification of the EXIT chart matching algorithm [10] of Section 9.2 was employed to jointly perform EXIT chart matching, while seeking a reduced IrVLC computational complexity by invoking component VLEC codebooks having a low RV-FDM. As a result, in Table 10.1, the computational complexity of the IrVLC-IrURC-low arrangement can be seen to be $25 \%$ lower than that of the IrVLC-IrURC-high arrangement, which was designed without seeking a reduced IrVLC computational complexity. Note that a reduced computational complexity could not be achieved when the IrVLC's EXIT function was matched to that of a regular URC, as discussed in Section 9.5.4. This was found to be because, unlike the ' $\mathrm{S}$ '-shaped inverted EXIT functions of the component VLEC codebooks having a high RV-FDM, those associated with a low RV-FDM do not rise rapidly enough to match with the URC EXIT function, which starts from a high point along the EXIT chart's $I_{e}^{i}$ axis.

In Section 7.2.1, we showed that the number of transitions employed by a symbol-based VLC trellis, and hence its computational complexity and memory requirement, scales with the square of the number of source symbols that it simultaneously decodes. For this reason, the total computational complexity and memory requirement can be reduced by decomposing each source symbol frame into sub-frames, which are decoded separately. However, owing to the nature of VLCs, the lengths of VLC-encoded transmission sub-frames typically vary from frame-to-frame. In order to facilitate their decoding in the receiver, the transmitter must convey the lengths of the sub-frames as explicit side information, which should be protected using a low-rate channel code, owing to its error sensitive nature. Hence, the choice of how many sub-frames to employ is a trade-off between the amount of side information required and the computational complexity as well as the memory requirements per source symbol. Note that the complexities provided in Table 10.1 and Figure 10.12 for the SBVLC- and SBIrVLC-TCM schemes of Section 7.2 are therefore specific to the particular considered case, in which each source symbol sub-frame comprised 100 symbols. In this arrangement, the required side information was found to account for $4 \%$ of the total information conveyed in Section 7.3.

By contrast, Section 7.2.1 showed that the number of transitions per source symbol employed by a bit-based trellis is independent of the number of source symbols that it simultaneously decodes. Hence, the total computational complexity and memory requirement cannot be reduced by decomposing each source symbol frame into sub-frames in this case. However, the memory required to decode each source symbol sub-frame will be reduced if more subframes are employed. If the sub-frames are decoded sequentially, this memory can be reused for each sub-frame and a lower-cost implementation will result. By contrast, the amount of 
memory required by an implementation that decodes all sub-frames concurrently will not be affected by the number of sub-frames employed.

In the schemes of Sections 8.5.4 and 9.6, the amount of side information required was significantly reduced by employing just a single source symbol sub-frame for each IrVLC component code. Using this approach, we found that less side information was required when a longer interleaver was employed, as described in Section 9.6. Indeed, the required side information was reduced to just $0.006 \%$ of the total information conveyed when a 1000000 bit interleaver was employed.

\subsection{Chapter ??: Iteratively Decoded VLC Space-Time Coded Modulation}

In this chapter an iteratively decoded variable length space-time coded modulation design was proposed. The joint design of source-coding, space-time coded modulation and iterative decoding was shown to achieve both spatial diversity and multiplexing gain, as well as coding and iteration gains at the same time. The variable length structure of the individual codewords mapped to the maximum of $N_{t}$ transmit antennas imposes no synchronisation and error propagation problems. The convergence properties of the proposed VL-STCM-ID scheme were analysed using 3D symbol-based EXIT charts as well as 2D EXIT chart projections. A significant iteration gain was achieved by the VL-STCM-ID scheme, which hence outperformed both the non-iterative VL-STCM scheme as well as the FL-STCM benchmarker with the aid of $N_{t}$ unity-rate recursive feedback precoders. The VL-STCM-ID scheme attains a near MIMO channel capacity performance.

\subsection{Chapter ??: Iterative Detection of Three-Stage Concatenated IrVLC FFH-MFSK}

In this chapter we investigated a serially concatenated IrvLC/FFH-MFSK Transceiver operating in a Rayleigh fading channel, when the transmitted signal was also corrupted by PBNJ. Our EXIT chart analysis demonstrated that a two-stage concatenated FFH-MFSK requires the employment of an additional unity-rate precoder for the sake of making the channel to appear recursive. For the sake of ensuring near-capacity operation, the IrVLC codec was specifically designed to ensure that the inverted EXIT curve of the IrVLC decoder matches the EXIT curve of the inner decoder. In this way, an open EXIT chart tunnel may be created even at low SNR values, providing source-correlation-dependent additional performance gains of up to $1.1 \mathrm{~dB}$ over the regular VLC-based benchmark scheme. Since the employment of the VLC involves non-identical occurence probabilities for the source symbols, it is not possible to provide a comparison of the proposed scheme with the state-of-the-art in the context of coded FFH-MFSK which traditionally employs equiprobable source symbols or bits. However, we have provided a comparison of the IrVLC scheme with a VLC scheme dispensing with the precoder; consequently we noted that the precoder-aided schemes yield a $E_{b} / N_{0}$ gain in excess of $7 \mathrm{~dB}$ over the system dispensing with the precoder, which suffers from an error floor when jamming is severe.

Moreover, we demonstrated that the 3-stage concatenation involving the demodulator, the rate-1 decoder and the outer IrVLC decoder yields superior performance compared to the 2-stage concatenation of the rate-1 decoder and the outer decoder. Naturally, the 3 -stage 
scheme imposes a higher complexity. By contrast, we found that a precoder of memory 1 is more suitable for the 3-stage IrVLC scheme, while the 2-stage scheme requires a precoder of memory 3 , thus the memory- 3 rate- 1 decoder imposes a somewhat higher complexity, than its memory- 1 counterpart.

In conclusion, the precoder-aided FFH-MFSK-VLC scheme constitutes a moderate-complexity design option, which can be employed in systems communicating through channels contaminated by PBNJ for transmission of joint source and channel encoded audio or video signals. If a higher complexity can be afforded, the IrVLC based scheme offers additional performance improvements. In our future work, we will investigate more sophisticated three-stage iterative decoding, exchanging extrinsic information amongst the demodulator, the rate- 1 decoder and the outer decoder.

\subsection{Future work}

As shown in Table 10.1, the schemes of Sections 6.2 and 7.2 employed a $R_{\mathrm{TCM}}=3 / 4$-rate TCM inner codec together with Set Partitioned (SP) $M_{\mathrm{TCM}}=16$-ary Quadrature Amplitude Modulation (16QAM) [129] to facilitate transmissions over an uncorrelated narrowband Rayleigh fading channel. However, in these schemes the maximum effective throughput is limited to $R_{\mathrm{TCM}} \cdot \log _{2}\left(M_{\mathrm{TCM}}\right)=3$ bits per channel use. Owing to the less-than-unity TCM coding rate, an effective throughput loss occurs for high channel $E_{b} / N_{0}$ values, where the capacity of the 16QAM modulated channel will exceed the maximum effective throughput of 3 bits per channel use and will approach $\log _{2}\left(M_{\mathrm{TCM}}\right)=4$ bits per channel use.

This motivated the employment of an inner URC codec in Section 8.4, which used $M_{\mathrm{BPSK}}=$ 2 Binary Phase Shift Keying (BPSK) [116] to facilitate transmissions over an uncorrelated narrowband Rayleigh fading channel, as shown in Figure 10.1. Here, the maximum effective throughput was equal to the maximum capacity of $\log _{2}\left(M_{\mathrm{BPSK}}\right)=1$ bit per channel use and no effective throughput loss was incurred. Indeed, the areas beneath the URC EXIT functions provided in Figure 8.13 were found to be equal to the corresponding channel capacities, as predicted by the area property of EXIT charts [19].

In the scheme of Section 9.4, we opted for employing a URC-based inner codec together with $M_{\mathrm{QAM}}=16 \mathrm{QAM}$ instead of BPSK, since this facilitates a higher maximum effective throughput of $\log _{2}\left(M_{\mathrm{QAM}}\right)=4$ bits per channel use. In Section 9.5.2 we showed that the receiver of Figure 9.3 would benefit from the iterative extrinsic information exchange of the 16QAM demodulator and the inner APP SISO decoder. However, for the sake of obtaining an implementational and computational complexity saving, the receiver of Figure 9.3 employed only the 'one-shot' activation of the 16QAM demodulator. However, as a result, when multiplied by $\log _{2}\left(M_{\mathrm{QAM}}\right)=4$, the average area beneath the URC EXIT functions exemplified in Figure 9.7 did not equal the corresponding channel capacities.

In Section 9.5.3, we defined the attainable capacity of a channel having a particular $E_{b} / N_{0}$ value as being equal to the average area beneath the corresponding URC EXIT functions, multiplied by $\log _{2}\left(M_{\mathrm{QAM}}\right)=4$. We showed that the channel's attainable capacity represents an upper bound to the maximum effective throughput for which an open EXIT chart tunnel can be achieved. This is because a scheme's effective throughput may be approximated by multiplying the area beneath the inverted outer EXIT function by $\log _{2}\left(M_{\mathrm{QAM}}\right)=4$ [19]. Since, this area must be lower than that beneath the inner EXIT function in order for an open EXIT chart to be facilitated, iterative decoding convergence to an infinitesimally low 
probability of error is prevented when the effective throughput is higher than the channel's attainable capacity. The discrepancy between the channel's capacity and its attainable capacity therefore imposes an effective throughput loss.

Section 9.5.2 showed that this effective throughput loss was minimised by employing Gray-coded 16QAM [141], since the corresponding EXIT function of Figure 9.8 is optimised to emerge from the highest possible point on the EXIT chart's $I_{e}^{m}$ axis. As shown in Figure 9.9, the effective throughput loss resulted in a discrepancy of $0.29 \mathrm{~dB}$ between the channel's $E_{b} / N_{0}$ capacity bound and its attainable capacity bound. Hence, the $0.17 \mathrm{~dB}$ discrepancy between the channel's attainable capacity bound and the $E_{b} / N_{0}$ value at which the IrVLC-IrURC-low arrangement could achieve a BER of $10^{-5}$ that is shown in Table 10.1, represents a $0.46 \mathrm{~dB}$ discrepancy from the channel's capacity bound.

In this section, we propose a method for mitigating the effective throughput loss of the IrVLC-IrURC scheme detailed in Section 9.4. However, this solution does not employ iterative extrinsic information exchange between the 16QAM demodulator and the inner APP SISO decoder. Instead, the benefit of iterative demodulation is mitigated by replacing the bit-based IrURC inner codec of Figure 9.3 with a Symbol Based Irregular Unity Code (SBIrURC). Unlike a bit-based IrURC, this SBIrURC can directly employ the symbol probabilities obtained for the demodulator's $M_{\mathrm{QAM}}=16$ constellation points without first converting them into sets of $\log _{2}\left(M_{\mathrm{QAM}}\right)=4$ bit probabilities. We refer to this proposed solution as the IrVLC-SBIrURC scheme and Figure 10.13 provides its schematic, which is reminiscent of the IrVLC-IrURC schematic provided in Figure 9.3.

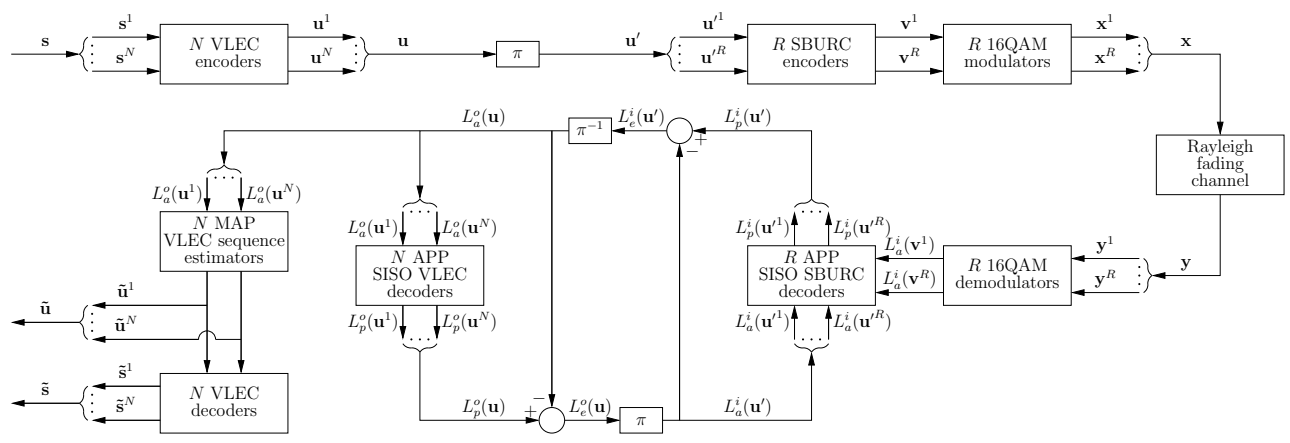

Figure 10.13: Schematic of the IrVLC-SBIrURC scheme.

In the IrVLC-SBIrURC scheme of Figure 10.13, IrVLC encoding, APP SISO decoding and MAP sequence estimation are performed in exactly the same way as in the IrVLCIrURC scheme of Figure 9.3. Furthermore, the source symbol frame $\mathbf{s}$, the transmission frame $\mathbf{u}$ and the LLR frames $L_{a}^{o}(\mathbf{u})$ as well as $L_{p}^{o}(\mathbf{u})$ are composed of $N$ number of subframes, as in the IrVLC-IrURC scheme of Figure 9.3. Likewise, the interleaved transmission frame $\mathbf{u}^{\prime}$ and the LLR frames $L_{a}^{i}\left(\mathbf{u}^{\prime}\right)$ as well as $L_{p}^{i}\left(\mathbf{u}^{\prime}\right)$ are composed of $R$ number of sub-frames, as before. Additionally, iterative decoding is performed as in the IrVLC-IrURC receiver of Figure 9.3, with the subtraction of the a priori LLR frames from the a posteriori LLR frames and the interleaving $\pi$ of the resultant extrinsic LLR frames, as shown in Figure 10.13. Finally, as in the IrVLC-IrURC scheme of Figure 9.3, the outer and inner EXIT 
functions of the IrVLC-SBIrURC scheme may be shaped by specifically selecting the fractions, $\left\{\alpha^{n}\right\}_{n=1}^{N}$ and $\left\{\alpha^{r}\right\}_{r=1}^{R}$, of the frames, $\mathbf{u}$ and $\mathbf{u}^{\prime}$, that are composed by the sub-frames, $\left\{\mathbf{u}^{n}\right\}_{n=1}^{N}$ and $\left\{\mathbf{u}^{\prime r}\right\}_{r=1}^{R}$, respectively. The IrVLC-SBIrURC scheme of Figure 10.13 differs from the IrVLC-IrURC scheme of Figure 9.3 in terms of the operation of the irregular inner codec and the modem.

In the IrVLC-SBIrURC transmitter of Figure 10.13, each interleaved transmission subframe $\mathbf{u}^{\prime r}$ is decomposed into sets of $\log _{2}\left(M_{\mathrm{QAM}}\right)=4$ consecutive bits, which are converted into $M_{\mathrm{QAM}}=16$-ary symbol values. In analogy with the IrVLC-IrURC scheme of Figure 9.3 , the $M_{\mathrm{QAM}}=16$-ary symbol values corresponding to each interleaved transmission sub-frame $\mathbf{u}^{\prime r}$ are encoded using a Symbol-Based Unity Rate Code (SBURC) having a different symbol-based Linear Feedback Shift Register (LFSR) design. For example, these LFSRs could employ the designs of Figure 9.6 if they were modified to employ modulo-16 additions and memory elements. Following SBURC encoding in the IrVLC-SBIrURC transmitter, the sub-frame $\mathbf{v}^{r}$ of SBURC-encoded $M_{\mathrm{QAM}}=16$-ary symbol values is obtained, as shown in Figure 10.13.

In the IrVLC-SBIrURC transmitter of Figure 10.13, the $M_{\mathrm{QAM}}=16$-ary symbol values of each SBURC-encoded sub-frame $\mathbf{v}^{r}$ are mapped to $M_{\mathrm{QAM}}=16 \mathrm{QAM}$ constellation points in order to generate the corresponding channel input symbol sub-frame $\mathbf{x}^{r}$. However, a different mapping scheme may be employed for each SBURC-encoded sub-frame $\mathbf{v}^{r}$, facilitating irregular modulation, as shown in Figure 10.13. Suitable $M_{\mathrm{QAM}}=16 \mathrm{QAM}$ mapping schemes include Gray coding [116], SP [129], Modified Set Partitioning (MSP) [206], the mixed mapping of [206], the Maximum Squared Euclidean Weight (MSEW) mapping of [207] and the $M 16^{a}$ and $M 16^{r}$ mappings of [208].

Following modulation, the resultant channel input symbol sub-frames $\left\{\mathbf{x}^{r}\right\}_{r=1}^{R}$ are concatenated in order to obtain the channel input symbol frame $\mathbf{x}$. This is transmitted over an uncorrelated narrowband Rayleigh fading channel and received as the channel output symbol frame $\mathbf{y}$, as shown in Figure 10.13. In the IrVLC-SBIrURC receiver of Figure 10.13, the channel output symbol frame $\mathbf{y}$ is decomposed into $R$ number of sub-frames $\left\{\mathbf{y}^{r}\right\}_{r=1}^{R}$, each of which is interpreted by a different $M_{\mathrm{QAM}}=16 \mathrm{QAM}$ demodulator. More specifically, for each channel output symbol, the demodulators determine the probability that the corresponding channel input symbol conveyed each of the $M_{\mathrm{QAM}}=16$ constellation points. Following this, the $M_{\mathrm{QAM}}=16$ probabilities associated with each channel output symbol in the sub-frame $\mathbf{y}^{r}$ are provided as a priori information to the corresponding APP SISO SBURC decoder by means of the Logarithmic A Posteriori Probability (Log-APP) sub-frame $L_{a}^{i}\left(\mathbf{v}^{r}\right)$, as shown in Figure 10.13.

In the IrVLC-SBIrURC receiver of Figure 10.13, each a priori LLR sub-frame $L_{a}^{i}\left(\mathbf{u}^{\prime r}\right)$ is decomposed into sets of $\log _{2}\left(M_{\mathrm{QAM}}\right)=4$ consecutive LLRs, which are converted into sets of $M_{\mathrm{QAM}}=16 \mathrm{Log}$-APPs in a manner similar to that of TCM [129]. The TCM symbolbased trellis [129] is employed to interpret these Log-APPs, together with those of the LogAPP sub-frame $L_{a}^{i}\left(\mathbf{v}^{r}\right)$ provided by the demodulator. Here, the BCJR algorithm [4] is employed to determine sets of $M_{\mathrm{QAM}}=16$ a posteriori Log-APPs, which are converted into sets of $\log _{2}\left(M_{\mathrm{QAM}}\right)=4$ LLRs for the a posteriori LLR sub-frame $L_{p}^{i}\left(\mathbf{u}^{\prime r}\right)$.

In addition to mitigating the effective throughput loss of the IrVLC-IrURC scheme of Figure 9.3, the IrVLC-SBIrURC scheme facilitates a higher degree of design freedom, owing to its employment of irregular modulation. While a different EXIT function may be obtained for 
each component URC code in the IrVLC-IrURC scheme of Figure 9.3, the IrVLC-SBIrURC scheme benefits from a different EXIT function for each combination of the component URC code and component 16QAM mapping. As a result, a greater variety of inner EXIT function shapes can be obtained, facilitating the improved joint matching of the inner and outer EXIT functions, as described in Section 9.3.

Note however, that APP SISO SBURC decoders are associated with a significantly higher computational complexity than their bit-based equivalents, owing to the significantly higher number of trellis transitions that they employ [129]. For example, a $M_{\mathrm{QAM}}=16$-ary SBURC employing just one memory element in its LFSR is associated with a trellis that employs $M_{\mathrm{QAM}}=16$ transitions from each of $M_{\mathrm{QAM}}=16$ states for each set of $\log _{2}\left(M_{\mathrm{QAM}}\right)=4$ bits. By contrast, the equivalent bit-based URC employs two transitions from each of two states for each bit. We can therefore expect the APP SISO decoder of the described SBURC to have a 16 times higher complexity than that of the equivalent bit-based URC. With reference to Table 9.7, we may observe that a 16 times increase in the inner APP SISO decoder's complexity would cause it to eclipse that of the outer APP SISO decoder and dominate the iterative decoding complexity. This could be countered, however, by employing the novel modification to the EXIT chart matching algorithm [10] of Section 9.2 for the sake of jointly perform EXIT chart matching while seeking a reduced SBIrURC computational complexity.

In the light of these discussions, our future work will consider the design and characterisation of the IrVLC-SBIrURC scheme of Figure 9.3.

\subsection{Closing remarks}

Throughout this book we have introduced novel IrVLC-aided wireless telecommunication schemes and methodologies for their design, in the pursuit of near-capacity operation. In Chapter 6, we developed a scheme without making any particular effort to facilitate its nearcapacity operation. Here, EXIT chart analysis was only employed in order to quantify how close to capacity the scheme may operate. By contrast, EXIT chart analysis was employed as an integral part of the design process in Chapter 7. More specifically, EXIT chart matching was employed to shape the IrVLC EXIT function to match that of the serially concatenated inner codec and hence to facilitate near-capacity operation. Further gains were achieved in Chapter 8 by challenging the conventional irregular coding design process of Figure 10.8. Instead of selecting a suite of IrVLC components having a wide variety of EXIT function shapes from a set of many candidates, a suite was directly designed using the RV-FDM of Section 8.2 and GA of Section 8.3. In Chapter 9, we invoked an irregular inner codec to complement the IrVLC, facilitating a higher degree of design freedom. This was exploited by the joint EXIT chart matching algorithm of Section 9.3 in order to match the IrVLC and inner EXIT functions to each other, facilitating even 'nearer-to-capacity' operation. Finally, in Section 10.12 outlining our future work, we proposed a method for mitigating the effective throughput loss that was associated with the scheme of Chapter 9, as well as for facilitating the employment of irregular modulation and for providing an even higher degree of design freedom. With these benefits, we may expect to achieve 'very-near-capacity' operation. 


\section{Bibliography}

[1] R. Bauer and J. Hagenauer, "Iterative source/channel-decoding using reversible variable length codes," in Proceedings of the IEEE Data Compression Conference, Snowbird, UT, USA, March 2000, pp. 93-102.

[2] — "Symbol by symbol MAP decoding of variable length codes," in Proceedings of the ITG Conference on Source and Channel Coding, Munich, Germany, January 2000, pp. 111-116.

[3] A. Viterbi, "Error bounds for convolutional codes and an asymptotically optimum decoding algorithm," IEEE Transactions on Information Theory, vol. 13, no. 2, pp. 260-269, April 1967.

[4] L. Bahl, J. Cocke, F. Jelinek, and J. Raviv, "Optimal decoding of linear codes for minimizing symbol error rate (Corresp.)," IEEE Transactions on Information Theory, vol. 20, no. 2, pp. 284-287, March 1974.

[5] R. G. Maunder, J. Kliewer, S. X. Ng, J. Wang, L.-L. Yang, and L. Hanzo, “Joint iterative decoding of trellis-based VQ and TCM," IEEE Transactions on Wireless Communications, vol. 6, no. 4, pp. 1327-1336, April 2007.

[6] _ - "Iterative joint video and channel decoding in a trellis-based vector-quantized video codec and trellis-coded modulation aided wireless videophone," in Proceedings of the IEEE Vehicular Technology Conference, Dallas, TX, USA, September 2005, pp. 922-926.

[7] Q. Qu, Y. Pei, and J. W. Modestino, "An adaptive motion-based unequal error protection approach for real-time video transport over wireless IP networks," IEEE Transactions on Multimedia, vol. 8, no. 5, pp. 1033-1044, October 2006.

[8] Y. C. Chang, S. W. Lee, and R. Komiya, "A low-complexity unequal error protection of H.264/AVC video using adaptive hierarchical QAM," IEEE Transactions on Consumer Electronics, vol. 52, no. 4, pp. 1153-1158, November 2006.

[9] T. Gan, L. Gan, and K.-K. Ma, "Reducing video-quality fluctuations for streaming scalable video using unequal error protection, retransmission, and interleaving," IEEE Transactions on Image Processing, vol. 15, no. 4, pp. 819-832, April 2006. 
[10] M. Tüchler and J. Hagenauer, "EXIT charts of irregular codes," in Proceedings of the Conference on Information Sciences and Systems, Princeton, NJ, USA, March 2002, pp. 748-753.

[11] R. G. Maunder, J. Wang, S. X. Ng, L.-L. Yang, and L. Hanzo, "On the performance and complexity of irregular variable length codes for near-capacity joint source and channel coding," to appear in IEEE Transactions on Wireless Communications.

[12] — , "Iteratively decoded irregular variable length coding and trellis coded modulation," in Proceedings of the IEEE Workshop on Signal Processing Systems, Shanghai, China, October 2007, pp. 222-227.

[13] S. Ahmed, R. G. Maunder, L.-L. Yang, S. X. Ng, and L. Hanzo, "Joint source coding, unity rate precoding and FFH-MFSK modulation using iteratively decoded irregular variable length coding," in Proceedings of the IEEE Vehicular Technology Conference, Baltimore, MD, USA, September 2007.

[14] M. El-Hajjar, R. G. Maunder, O. Alamri, S. X. Ng, and L. Hanzo, "Iteratively decoded irregular variable length coding and sphere-packing modulation-aided differential space-time spreading," in Proceedings of the IEEE Vehicular Technology Conference, Baltimore, MD, USA, September 2007.

[15] R. G. Maunder and L. Hanzo, "Genetic algorithm aided design of variable length error correcting codes for arbitrary coding rate and error correction capability," accepted by IEEE Transactions on Communications.

[16] _ _ "Genetic algorithm aided design of near-capacity irregular variable length codes," submitted to IEEE Wireless Communications and Networking Conference, Las Vegas, NV, USA, March 2008.

[17] — , "Near-capacity irregular variable length coding and irregular unity rate coding," accepted by IEEE Transactions on Wireless Communications.

[18] — - "Concatenated irregular variable length coding and irregular unity rate coding," submitted to IEEE International Conference on Communications, Beijing, China, May 2008.

[19] A. Ashikhmin, G. Kramer, and S. ten Brink, "Code rate and the area under extrinsic information transfer curves," in Proceedings of the IEEE International Symposium on Information Theory, Lausanne, Switzerland, June 2002, p. 115.

[20] V. Buttigieg, "Variable-Length Error-Correcting Codes," Ph.D. dissertation, Department of Electronic Enginnering, University of Manchester, Manchester, U.K., 1995.

[21] C. Lamy and J. Paccaut, "Optimised constructions for variable-length error correcting codes," in Proceedings of the IEEE Information Theory Workshop, Paris, France, March 2003, pp. 183186.

[22] J. Wang, L.-L. Yang, and L. Hanzo, "Iterative construction of reversible variable-length codes and variable-length error-correcting codes," IEEE Communications Letters, vol. 8, no. 11, pp. 671-673, November 2004. 
[23] R. Y. Tee, R. G. Maunder, J. Wang, and L. Hanzo, "Capacity approaching irregular bitinterleaved coded modulation," submitted to IEEE Vehicular Technology Conference, Marina Bay, Singapore, May 2008.

[24] C. E. Shannon, "The mathematical theory of communication," Bell System Technical Journal, vol. 27, pp. 379-423, July 1948.

[25] C. Shannon, "A mathematical theory of communication - part I," Bell Systems Technical Journal, pp. 379-405, 1948.

[26] — , "A mathematical theory of communication — part II," Bell Systems Technical Journal, pp. 405-423, 1948.

[27] — , "A mathematical theory of communication — part III," Bell Systems Technical Journal, vol. 27, pp. 623-656, 1948.

[28] H. Nyquist, "Certain factors affecting telegraph speed," Bell System Technical Journal, p. 617, April 1928.

[29] R. Hartley, “Transmission of information,” Bell Systems Technical Journal, p. 535, 1928.

[30] N. Abramson, Information Theory and Coding. New York: McGraw-Hill, 1975.

[31] A. Carlson, Communication Systems. New York: McGraw-Hill, 1975.

[32] H. Raemer, Statistical Communication Theory and Applications. Englewood Cliffs, NJ: Prentice-Hall, 1969.

[33] P. Ferenczy, Telecommunications Theory, in Hungarian: Hirkozleselmelet. Budapest, Hungary: Tankonyvkiado, 1972.

[34] K. Shanmugam, Digital and Analog Communications Systems. New York: John Wiley and Sons, 1979.

[35] C. Shannon, "Communication in the presence of noise," Proceedings of the I.R.E., vol. 37, pp. 10-22, January 1949.

[36] - "Probability of error for optimal codes in a Gaussian channel," Bell Systems Technical Journal, vol. 38, pp. 611-656, 1959.

[37] A. Jain, Fundamentals of Digital Image Processing. Englewood Cliffs, NJ: Prentice-Hall, 1989.

[38] A. Hey and R. Allen, Eds., R.P. Feynman: Feynman Lectures on Computation. Reading, MA: Addison-Wesley, 1996.

[39] C. Berrou, A. Glavieux and P. Thitimajshima, "Near Shannon Limit Error-Correcting Coding and Decoding: Turbo Codes," in Proceedings of the International Conference on Communications, Geneva, Switzerland, May 1993, pp. 1064-1070.

[40] L. Hanzo, W. Webb, and T. Keller, Single- and Multi-Carrier Quadrature Amplitude Modulation: Principles and Applications for Personal Communications, WLANs and Broadcasting. IEEE Press, 2000. 
[41] J. Hagenauer, "Quellengesteuerte kanalcodierung fuer sprach- und tonuebertragung im mobilfunk," Aachener Kolloquium Signaltheorie, pp. 67-76, 23-25 March 1994.

[42] A. Viterbi, "Wireless digital communications: A view based on three lessons learned," IEEE Communications Magazine, pp. 33-36, September 1991.

[43] L. Hanzo and J. Woodard, "An intelligent multimode voice communications system for indoor communications," IEEE Transactions on Vehicular Technology, vol. 44, no. 4, pp. 735-748, November 1995

[44] L. Hanzo, R. Salami, R. Steele, and P. Fortune, "Transmission of digitally encoded speech at 1.2 Kbaud for PCN,” IEE Proceedings, Part I, vol. 139, no. 4, pp. 437-447, August 1992.

[45] D. J. Costello and G. D. Forney, "Channel coding: The road to channel capacity," Proceedings of the IEEE, vol. 95, no. 6, pp. 1150-1177, June 2007.

[46] I. Jacobs and E. Berlekamp, "A lower bound to the distribution of computation for sequential decoding," IEEE Transactions on Information Theory, vol. 13, no. 2, pp. 167-174, April 1967.

[47] J. Wozencraft, "Sequential decoding for reliable communication," IRE National Convention Record, vol. 5, no. 2, pp. 11-25, August 1957.

[48] J. M. Wozencraft and B. Reiffen, Sequential Decoding. Cambridge, Mass.: MIT Press, 1961.

[49] J. Massey, Threshold Decoding. Cambridge, MA, USA: MIT Press, 1961.

[50] R. Fano, "A heuristic discussion of probabilistic decoding," IEEE Transactions on Information Theory, vol. 9, no. 2, pp. 64-74, April 1963.

[51] P. Elias, "Coding for noisy channels," IRE International Convention Record, vol. 3, no. 4, pp. $37-46,1955$.

[52] G. D. Forney, "Review of random tree codes," NASA Ames Research Center, Moffett Field, CA, USA, Tech. Rep. NASA CR73176, December 1967.

[53] C. Berrou, A. Glavieux, and P. Thitimajshima, "Near Shannon limit error-correcting coding and decoding: Turbo-codes (1)," in Proceedings of the IEEE International Conference on Communications, vol. 2, Geneva, May 1993, pp. 1064-1070.

[54] R. G. Gallager, "Low-density parity-check codes," IRE Transactions on Information Theory, vol. 8, no. 1, pp. 21-28, January 1962.

[55] — _ Low Density Parity Check Codes. Cambridge, Mass.: MIT Press, 1963.

[56] D. J. C. MacKay and R. M. Neal, "Near Shannon limit performance of low density parity check codes," Electronics Letters, vol. 32, no. 18, pp. 457-458, August 1996.

[57] R. Tanner, "A recursive approach to low complexity codes," IEEE Transactions on Information Theory, vol. 27, no. 5, pp. 533-547, September 1981.

[58] M. Luby, M. Mitzenmacher, A. Shokrollahi, D. Spielman, and V. Stemann, "Practical lossresilient codes," in Proceedings of the ACM Symposium on Theory of Computing, El Paso, TX, USA, May 1997, pp. 150-159. 
[59] B. J. Frey and D. J. C. MacKay, "Irregular turbo-like codes," in Proceedings of the International Symposium on Turbo Codes, Brest, France, September 2000, pp. 67-72.

[60] A. J. Viterbi and J. K. Omura, Principles of Digital Communications and Coding. New York, NY, USA: McGraw-Hill, 1979.

[61] L. Hanzo, P. J. Cherriman, and J. Streit, Video Compression and Communications: H.261, H.263, H.264, MPEG4 and Proprietary Codecs for HSDPA-Style Adaptive Turbo-Transceivers. John Wiley and IEEE Press, September 2007.

[62] L. Hanzo, F. C. A. Somerville, and J. P. Woodard, Voice and Audio Compression for Wireless Communications. John Wiley and IEEE Press, 2007.

[63] N. Ahmed, T. Natarajan, and K. R. Rao, "Discrete cosine transform," IEEE Transactions on Computers, vol. C-23, pp. 90-93, January 1974.

[64] A. N. Netravali and J. D. Robbins, "Motion-compensated television coding. I," Bell System Technical Journal, vol. 58, pp. 631-670, March 1979.

[65] D. A. Huffman, "A method for the construction of minimum-redundancy codes," Proceedings of the IRE, vol. 40, pp. 1098-1101, September 1952.

[66] Information technology - Coding of moving pictures and associated audio for digital storage media at up to about 1.5 Mbit/s, ISO/IEC Std. 11 172, June 1996.

[67] Information technology - Generic coding of moving pictures and associated audio information, ISO/IEC Std. 13818

[68] Information technology - Coding of audio-visual objects, ISO/IEC Std. 14496.

[69] Video codec for audiovisual services at $p \times 64 \mathrm{kbit} / \mathrm{s}$, ITU-T Std. H.261, March 1993.

[70] Video coding for low bit rate communication, ITU-T Std. H.263, January 2005.

[71] Advanced video coding for generic audiovisual services, ITU-T Std. H.264, March 2005.

[72] J. L. Massey, "Joint source and channel coding," in Communication Systems and Random Process Theory, J. K. Skwirzynski, Ed. Amsterdam, The Netherlands: Sijthoff and Noordhoff, December 1978, pp. 279-293.

[73] R. E. van Dyck and D. J. Miller, "Transport of wireless video using separate, concatenated, and joint source-channel coding," Proceedings of the IEEE, vol. 87, no. 10, pp. 1734-1750, October 1999.

[74] S. Lloyd, "Least squares quantization in PCM," IEEE Transactions on Information Theory, vol. 28, no. 2, pp. 129-137, March 1982.

[75] J. Max, "Quantizing for minimum distortion," IRE Transactions on Information Theory, vol. 6, no. 1, pp. 7-12, March 1960.

[76] A. Kurtenbach and P. Wintz, "Quantizing for noisy channels," IEEE Transactions on Communications, vol. 17, no. 2, pp. 291-302, April 1969. 
[77] N. Farvardin and V. Vaishampayan, "Optimal quantizer design for noisy channels: An approach to combined source-channel coding," IEEE Transactions on Information Theory, vol. 33, no. 6, pp. 827-838, November 1987.

[78] H. Kumazawa, M. Kasahara, and T. Namekawa, "A construction of vector quantizers for noisy channels," Electronics and Engineering in Japan, vol. 67-B, no. 4, pp. 39-47, January 1984.

[79] N. Farvardin, "A study of vector quantization for noisy channels," IEEE Transactions on Information Theory, vol. 36, no. 4, pp. 799-809, July 1990.

[80] Y. Linde, A. Buzo, and R. Gray, “An algorithm for vector quantizer design,” IEEE Transactions on Communications, vol. 28, no. 1, pp. 84-95, January 1980.

[81] K. Sayood and J. C. Borkenhagen, "Use of residual redundancy in the design of joint source/channel coders," IEEE Transactions on Communications, vol. 39, no. 6, pp. 838-846, June 1991.

[82] N. Phamdo and N. Farvardin, "Optimal detection of discrete Markov sources over discretememoryless channels - applications to combined source-channel coding," IEEE Transactions on Information Theory, vol. 40, no. 1, pp. 186-193, January 1994.

[83] T. Fingscheidt and P. Vary, "Softbit speech decoding: A new approach to error concealment," IEEE Transactions on Speech and Audio Processing, vol. 9, no. 3, pp. 240-251, March 2001.

[84] J. Kliewer and R. Thobaben, "Iterative joint source-channel decoding of variable-length codes using residual source redundancy," IEEE Transactions on Wireless Communications, vol. 4, no. 3, pp. 919-929, May 2005.

[85] F. Lahouti and A. K. Khandani, "Soft reconstruction of speech in the presence of noise and packet loss," IEEE Transactions on Audio, Speech and Language Processing, vol. 15, no. 1, pp. 44-56, January 2007.

[86] R. Thobaben and J. Kliewer, "Low-complexity iterative joint source-channel decoding for variable-length encoded Markov sources," IEEE Transactions on Communications, vol. 53, no. 12, pp. 2054-2064, December 2005.

[87] D. J. Miller and M. Park, "A sequence-based approximate MMSE decoder for source coding over noisy channels using discrete hidden Markov models," IEEE Transactions on Communications, vol. 46, no. 2, pp. 222-231, February 1998.

[88] M. W. Marcellin and T. R. Fischer, "Trellis coded quantization of memoryless and Gauss-Markov sources," IEEE Transactions on Communications, vol. 38, no. 1, pp. 82-93, January 1990.

[89] V. Buttigieg and P. G. Farrell, "Variable-length error-correcting codes," IEE Proceedings on Communications, vol. 147, no. 4, pp. 211-215, August 2000.

[90] V. B. Balakirsky, "Joint source-channel coding with variable length codes," in Proceedings of the IEEE International Symposium on Information Theory, Ulm, Germany, June 1997, p. 419.

[91] M. Park and D. J. Miller, "Decoding entropy-coded symbols over noisy channels by MAP sequence estimation for asynchronous HMMs," in Proceedings of the Conference on Information Sciences and Systems, Princeton, NJ, USA, March 1998, pp. 477-482. 
[92] M. Bystrom, S. Kaiser, and A. Kopansky, "Soft source decoding with applications," IEEE Transactions on Circuits and Systems for Video Technology, vol. 11, no. 10, pp. 1108-1120, October 2001.

[93] N. Görtz, "A generalized framework for iterative source-channel decoding," Annals of Telecommunications, pp. 435-446, July/August 2001.

[94] J. Hagenauer and N. Görtz, "The turbo principle in joint source-channel coding," in Proceedings of the IEEE Information Theory Workshop, Paris, France, March 2003, pp. 275-278.

[95] X. Jaspar, C. Guillemot, and L. Vandendorpe, "Joint source-channel turbo techniques for discrete-valued sources: From theory to practice," Proceedings of the IEEE, vol. 95, no. 6, pp. 1345-1361, June 2007.

[96] J. Modestino and D. Daut, "Combined source-channel coding of images," IEEE Transactions on Communications, vol. 27, no. 11, pp. 1644-1659, November 1979.

[97] M. Grangetto, B. Scanavino, G. Olmo, and S. Benedetto, "Iterative decoding of serially concatenated arithmetic and channel codes with JPEG 2000 applications," IEEE Transactions on Image Processing, vol. 16, no. 6, pp. 1557-1567, June 2007.

[98] J. B. MacQueen, "Some methods for classification and analysis of multivariate observations," in Proceedings of the Berkeley Symposium on Mathematical Statistics and Probability, CA, USA, March 1966, pp. 281-297.

[99] Y. Takishima, M. Wada, and H. Murakami, "Reversible variable length codes," IEEE Transactions on Communications, vol. 43, no. 234, pp. 158-162, February 1995.

[100] J. Wen and J. D. Villasenor, "Reversible variable length codes for efficient and robust image and video coding," in Proceedings of the Data Compression Conference, Snowbird, UT, USA, March 1998, pp. 471-480.

[101] C.-W. Tsai and J.-L. Wu, "On constructing the Huffman-code-based reversible variable-length codes," IEEE Transactions on Communications, vol. 49, no. 9, pp. 1506-1509, September 2001.

[102] C.-W. Lin, Y.-J. Chuang, and J.-L. Wu, "Generic construction algorithms for symmetric and asymmetric RVLCs," in Proceedings of the International Conference on Communication Systems, vol. 2, Singapore, November 2002, pp. 968-972.

[103] K. Lakovic and J. Villasenor, "On design of error-correcting reversible variable length codes," IEEE Communications Letters, vol. 6, no. 8, pp. 337-339, August 2002.

[104] V. Buttigieg and P. G. Farrell, "On variable-length error-correcting codes," in Proceedings of the IEEE International Symposium on Information Theory, Trondheim, Norway, June 1994, p. 507.

[105] V. B. Balakirsky, "Joint source-channel coding using variable-length codes," Problems of Information Transmission, vol. 37, no. 1, pp. 10-23, January 2001.

[106] R. Bauer and J. Hagenauer, "On variable length codes for iterative source/channel decoding," in Proceedings of the IEEE Data Compression Conference, Snowbird, UT, USA, March 2001, pp. 273-282. 
[107] G. D. Forney, “The Viterbi algorithm,” Proceedings of the IEEE, vol. 61, no. 3, pp. 268-278, March 1973.

[108] L. Perros-Meilhac and C. Lamy, "Huffman tree based metric derivation for a low-complexity sequential soft VLC decoding," in Proceedings of the IEEE International Conference on Communications, vol. 2, New York, NY, USA, April 2002, pp. 783-787.

[109] A. Guyader, E. Fabre, C. Guillemot, and M. Robert, "Joint source-channel turbo decoding of entropy-coded sources," IEEE Journal on Selected Areas in Communications, vol. 19, no. 9, pp. 1680-1696, September 2001.

[110] N. Demir and K. Sayood, "Joint source/channel coding for variable length codes," in Proceedings of the IEEE Data Compression Conference, Snowbird, UT, USA, March 1998, pp. 139-148.

[111] M. Park and D. J. Miller, "Joint source-channel decoding for variable-length encoded data by exact and approximate MAP sequence estimation," IEEE Transactions on Communications, vol. 48, no. 1, pp. 1-6, January 2000.

[112] C. Lamy and O. Pothier, "Reduced complexity maximum a posteriori decoding of variablelength codes," in Proceedings of the IEEE Global Telecommunications Conference, vol. 2, San Antonio, TX, USA, November 2001, pp. 1410-1413.

[113] R. Thobaben and J. Kliewer, "Robust decoding of variable-length encoded Markov sources using a three-dimensional trellis," IEEE Communications Letters, vol. 7, no. 7, pp. 320-322, July 2003.

[114] J. Hagenauer, "The turbo principle - Tutorial introduction and state of the art," in Proceedings of the International Symposium on Turbo Codes, Brest, France, September 1997, pp. 1-11.

[115] L. Hanzo, J. P. Woodard, and P. Robertson, "Turbo decoding and detection for wireless applications," Proceedings of the IEEE, vol. 95, no. 6, pp. 1178-1200, June 2007.

[116] L. Hanzo, S. X. Ng, T. Keller, and W. Webb, Quadrature Amplitude Modulation. Chichester, UK: Wiley, 2004.

[117] C. Guillemot and P. Siohan, "Joint source-channel decoding of variable-length codes with soft information: A survey," EURASIP Journal on Applied Signal Processing, pp. 906-927, June 2005.

[118] J. Hagenauer and P. Hoeher, "A Viterbi algorithm with soft-decision outputs and its applications," in Proceedings of the IEEE Global Telecommunications Conference, Dallas, TX, USA, November 1989, pp. 1680-1686.

[119] E. Boutillon, C. Douillard, and G. Montorsi, "Iterative decoding of concatenated convolutional codes: Implementation issues," Proceedings of the IEEE, vol. 95, no. 6, pp. 1201-1227, June 2007.

[120] P. Robertson, E. Villebrun, and P. Hoeher, "A comparison of optimal and sub-optimal MAP decoding algorithms operating in the log domain," in Proceedings of the IEEE International Conference on Communications, vol. 2, Seattle, WA, USA, June 1995, pp. 1009-1013.

[121] V. Franz and J. B. Anderson, "Concatenated decoding with a reduced-search BCJR algorithm," IEEE Journal on Selected Areas in Communications, vol. 16, no. 2, pp. 186-195, February 1998. 
[122] B. Vucetic, Y. Li, L. C. Perez, and F. Jiang, "Recent advances in turbo code design and theory," Proceedings of the IEEE, vol. 95, no. 6, pp. 1323-1344, June 2007.

[123] K. Gracie and M.-H. Hamon, "Turbo and turbo-like codes: Principles and applications in telecommunications," Proceedings of the IEEE, vol. 95, no. 6, pp. 1228-1254, June 2007.

[124] J. Hagenauer, E. Offer, and L. Papke, "Iterative decoding of binary block and convolutional codes," IEEE Transactions on Information Theory, vol. 42, no. 2, pp. 429-445, March 1996.

[125] S. Benedetto and G. Montorsi, "Unveiling turbo codes: Some results on parallel concatenated coding schemes," IEEE Transactions on Information Theory, vol. 42, no. 2, pp. 409-428, March 1996.

[126] R. M. Pyndiah, "Near-optimum decoding of product codes: Block turbo codes," IEEE Transactions on Communications, vol. 46, no. 8, pp. 1003-1010, August 1998.

[127] I. S. Reed and G. Solomon, "Polynomial codes over certain finite fields," Journal of the Society for Industrial and Applied Mathematics, vol. 8, no. 2, pp. 300-304, June 1960.

[128] P. Robertson and T. Worz, "A novel bandwidth efficient coding scheme employing turbo codes," in Proceedings of the IEEE International Conference on Communications, vol. 2, Dallas, TX, USA, June 1996, pp. 962-967.

[129] G. Ungerboeck, "Channel coding with multilevel/phase signals," IEEE Transactions on Information Theory, vol. 28, no. 1, pp. 55-67, January 1982.

[130] J. Kliewer and R. Thobaben, "Parallel concatenated joint source-channel coding," Electronics Letters, vol. 39, pp. 1664-6, November 2003.

[131] S. Benedetto and G. Montorsi, "Serial concatenation of block and convolutional codes," Electronics Letters, vol. 32, no. 10, pp. 887-888, May 1996.

[132] — - "Iterative decoding of serially concatenated convolutional codes," Electronics Letters, vol. 32, no. 13, pp. 1186-1188, June 1996.

[133] N. Görtz, "On the iterative approximation of optimal joint source-channel decoding," IEEE Journal on Selected Areas in Communications, vol. 19, no. 9, pp. 1662-1670, September 2001.

[134] M. Adrat, J.-M. Picard, and P. Vary, "Softbit-source decoding based on the turbo-principle," in Proceedings of the IEEE Vehicular Technology Conference, vol. 4, Atlantic City, NJ, USA, October 2001, pp. 2252-2256.

[135] J. Kliewer and R. Thobaben, "Combining FEC and optimal soft-input source decoding for the reliable transmission of correlated variable-length encoded signals," in Proceedings of the IEEE Data Compression Conference, Snowbird, UT, USA, April 2002, pp. 83-91.

[136] S. X. Ng, F. Guo, J. Wang, L.-L. Yang, and L. Hanzo, "Joint source-coding, channel-coding and modulation schemes for AWGN and Rayleigh fading channels," Electronics Letters, vol. 39, no. 17, pp. 1259-1261, August 2003.

[137] D. Divsalar, H. Jin, and R. McEliece, "Coding theorems for 'turbo-like' codes," in Proceedings of the Allerton Conference on Communications, no. 36, Allerton, IL, USA, September 1998, pp. 201-210. 
[138] C. Douillard, M. Jezequel, C. Berrou, A. Picart, P. Didier, and A. Glavieux, "Iterative correction of intersymbol interference: Turbo equalization," European Transactions on Telecommunications, vol. 6, pp. 507-511, September 1995.

[139] X. Li and J. A. Ritcey, "Bit-interleaved coded modulation with iterative decoding," in Proceedings of the IEEE International Conference on Communications, vol. 2, Vancouver, BC, Canada, June 1999, pp. 858-863.

[140] S. ten Brink, J. Speidel, and R.-H. Yan, "Iterative demapping and decoding for multilevel modulation," in Proceedings of the IEEE Global Telecommunications Conference, vol. 1, Sydney,NSW, Australia, November 1998, pp. 579-584.

[141] G. Caire, G. Taricco, and E. Biglieri, "Bit-interleaved coded modulation," IEEE Transactions on Information Theory, vol. 44, no. 3, pp. 927-946, May 1998.

[142] J. C. Serrato and T. O'Farrell, "Joint demapping and source decoding for multilevel modulation," in Proceedings of the Wireless Communications and Networking Conference, vol. 4, Las Vegas, NV, USA, April 2006, pp. 2140-2144.

[143] S. Benedetto, D. Divsalar, G. Montorsi, and F. Pollara, "Serial concatenated trellis coded modulation with iterative decoding," in Proceedings of the IEEE International Symposium on Information Theory, Ulm, Germany, June/July 1997, p. 8.

[144] D. Divsalar, S. Dolinar, and F. Pollara, "Serial concatenated trellis coded modulation with rate-1 inner code," in Proceedings of the IEEE Global Telecommunications Conference, vol. 2, San Francisco, CA, USA, November 2000, pp. 777-782.

[145] M. Moher, "An iterative multiuser decoder for near-capacity communications," IEEE Transactions on Communications, vol. 46, no. 7, pp. 870-880, July 1998.

[146] W.-J. Choi, K.-W. Cheong, and J. M. Cioffi, "Iterative soft interference cancellation for multiple antenna systems," in Proceedings of the IEEE Wireless Communications and Networking Conference, vol. 1, Chicago, IL, USA, September 2000, pp. 304-309.

[147] J. Wang, S. X. Ng, A. Wolfgang, L.-L. Yang, S. Chen, and L. Hanzo, "Near-capacity three-stage MMSE turbo equalization using irregular convolutional codes," in Proceedings of the International Symposium on Turbo Codes, Munich, Germany, April 2006, electronic publication.

[148] M. Tüchler, "Convergence prediction for iterative decoding of threefold concatenated systems," in Proceedings of the IEEE Global Telecommunications Conference, vol. 2, Taipei, Taiwan, November 2002, pp. 1358-1362.

[149] J. Hagenauer and P. Hoeher, "Concatenated Viterbi decoding," in Proceedings of the Joint Swedish-Soviet International Workshop on Information Theory, Gotland, Sweden, September 1989, pp. 29-33.

[150] D. Divsalar, S. Dolinar, and F. Pollara, "Low complexity turbo-like codes," in Proceedings of the International Symposium on Turbo Codes, Brest, France, September 2000, pp. 78-80. 
[151] M. Tüchler, S. ten Brink, and J. Hagenauer, "Measures for tracing convergence of iterative decoding algorithms," in Proceedings of the ITG Conference on Source and Channel Coding, Berlin, Germany, January 2002, pp. 53-60.

[152] S. ten Brink, "Convergence of iterative decoding," Electronics Letters, vol. 35, no. 10, pp. 806808, May 1999.

[153] I. Land, P. Hoeher, and S. Gligorević, "Computation of symbol-wise mutual information in transmission systems with log APP decoders and application to EXIT charts," in Proceedings of the International ITG Conference on Source and Channel Coding, Erlangen, Germany, January 2004, pp. 195-202.

[154] J. Hokfelt, O. Edfors, and T. Maseng, "A turbo code interleaver design criterion based on the performance of iterative decoding," IEEE Communications Letters, vol. 5, no. 2, pp. 52-54, February 2001.

[155] S. Dolinar and D. Divsalar, "Weight distributions for turbo codes using random and nonrandom permutations," Telecommunications and Data Acquisition Progress Report, vol. 122, pp. 56-65, April 1995.

[156] W. Feng, J. Yuan, and B. S. Vucetic, "A code-matched interleaver design for turbo codes," IEEE Transactions on Communications, vol. 50, no. 6, pp. 926-937, June 2002.

[157] J. Hokfelt, O. Edfors, and T. Maseng, "Turbo codes: correlated extrinsic information and its impact on iterative decoding performance," in Proceedings of the IEEE Vehicular Technology Conference, vol. 3, Houston, TX, USA, July 1999, pp. 1871-1875.

[158] A. Ashikhmin, G. Kramer, and S. ten Brink, "Extrinsic information transfer functions: Model and erasure channel properties," IEEE Transactions on Information Theory, vol. 50, no. 11, pp. 2657-2673, November 2004.

[159] J. Kliewer, A. Huebner, and D. J. Costello, "On the achievable extrinsic information of inner decoders in serial concatenation," in Proceedings of the IEEE International Symposium on Information Theory, Seattle, WA, USA, July 2006, pp. 2680-2684.

[160] R. Thobaben, "EXIT functions for randomly punctured systematic codes," in Proceedings of the IEEE Information Theory Workshop, Lake Tahoe, CA, USA, September 2007, pp. 24-29.

[161] J. Kliewer, N. Görtz, and A. Mertins, "Iterative source-channel decoding with Markov random field source models," IEEE Transactions on Signal Processing, vol. 54, no. 10, pp. 3688-3701, October 2006.

[162] R. Thobaben and J. Kliewer, "Design considerations for iteratively-decoded source-channel coding schemes," in Proceedings of the Allerton Conference on Communications, Control, and Computing, Monticello, IL, USA, September 2006.

[163] J. Kliewer, S. X. Ng, and L. Hanzo, "Efficient computation of EXIT functions for nonbinary iterative decoding," IEEE Transactions on Communications, vol. 54, no. 12, pp. 2133-2136, December 2006. 
[164] M. Adrat, J. Brauers, T. Clevorn, and P. Vary, "The EXIT-characteristic of softbit-source decoders," IEEE Communications Letters, vol. 9, no. 6, pp. 540-542, June 2005.

[165] J. G. Proakis, Digital Communications. McGraw-Hill, 1983.

[166] T. J. Richardson, M. A. Shokrollahi, and R. L. Urbanke, "Design of capacity-approaching irregular low-density parity-check codes," IEEE Transactions on Information Theory, vol. 47, no. 2, pp. 619-637, February 2001.

[167] G. Yue, X. Wang, and M. Madihian, "Design of rate-compatible irregular repeat accumulate codes," IEEE Transactions on Communications, vol. 55, no. 6, pp. 1153-1163, June 2007.

[168] G. Yue, B. Lu, and X. Wang, "Analysis and design of finite-length LDPC codes," IEEE Transactions on Vehicular Technology, vol. 56, no. 3, pp. 1321-1332, May 2007.

[169] J. Xu, L. Chen, I. Djurdjevic, S. Lin, and K. Abdel-Ghaffar, "Construction of regular and irregular LDPC codes: Geometry decomposition and masking," IEEE Transactions on Information Theory, vol. 53, no. 1, pp. 121-134, January 2007.

[170] S. Tong, S. Zhang, B. Bai, and X. Wang, "Fast encodable and decodable irregular repeat accumulate codes from circulant permutation matrices," Electronics Letters, vol. 43, pp. 48-49, January 2007.

[171] A. Mahmood and E. Jaffrot, "Greedy check allocation for irregular LDPC codes optimization in multicarrier systems," in Proceedings of the Wireless Communications and Networking Conference, Hong Kong, March 2007, pp. 687-691.

[172] J. Chen, R. M. Tanner, J. Zhang, and M. P. C. Fossorier, "Construction of irregular LDPC codes by quasi-cyclic extension,” IEEE Transactions on Information Theory, vol. 53, no. 4, pp. 14791483, April 2007.

[173] H. Chen and Z. Cao, "A modified PEG algorithm for construction of LDPC codes with strictly concentrated check-node degree distributions," in Proceedings of the Wireless Communications and Networking Conference, Hong Kong, March 2007, pp. 564-568.

[174] A. Abbasfar, D. Divsalar, and K. Yao, "Accumulate-repeat-accumulate codes," IEEE Transactions on Communications, vol. 55, no. 4, pp. 692-702, April 2007.

[175] M. Tüchler, "Design of serially concatenated systems depending on the block length," IEEE Transactions on Communications, vol. 52, no. 2, pp. 209-218, February 2004.

[176] A. Q. Pham, J. Wang, L.-L. Yang, and L. Hanzo, "An iterative detection aided unequal error protection wavelet video scheme using irregular convolutional codes," in Proceedings of the IEEE Vehicular Technology Conference, vol. 5, Melbourne, Australia, May 2006, pp. 24842488.

[177] O. Alamri, J. Wang, S. X. Ng, L.-L. Yang, and L. Hanzo, "Near-capacity three-stage turbo detection of irregular convolutional coded joint sphere-packing modulation and space-time coding," in Proceedings of the IEEE International Conference on Communications, Glasgow, UK, June 2007. 
[178] J. Wang, N. S. Othman, J. Kliewer, L.-L. Yang, and L. Hanzo, "Turbo-detected unequal error protection irregular convolutional codes designed for the wideband advanced multirate speech codec," in Proceedings of the IEEE Vehicular Technology Conference, vol. 2, Dallas, TX, USA, September 2005, pp. 927-931.

[179] S. Tan, J. Wang, S. X. Ng, S. Chen, and L. Hanzo, "Three-stage turbo MBER multiuser beamforming receiver using irregular convolutional codes," to appear in IEEE Transactions on Vehicular Technology.

[180] N. Wu, O. Alamri, S. X. Ng, and L. Hanzo, "Precoded sphere packing aided bit-interleaved differential space-time coded modulation using iterative decoding," to appear in IEEE Transactions on Vehicular Technology.

[181] S. X. Ng, R. G. Maunder, J. Wang, L.-L. Yang, and L. Hanzo, “Joint iterative-detection of reversible variable-length coded constant bit rate vector-quantized video and coded modulation," in Proceedings of the European Signal Processing Conference, Vienna, Austria, September 2004, pp. 2231-2234.

[182] S. X. Ng, J. Y. Chung, F. Guo, and L. Hanzo, "A turbo-detection aided serially concatenated MPEG-4/TCM videophone transceiver," in Proceedings of the IEEE Vehicular Technology Conference, vol. 4, Los Angeles, CA, USA, September 2004, pp. 2606-2610.

[183] C. Bergeron and C. Lamy-Bergot, "Soft-input decoding of variable-length codes applied to the H.264 standard," in Proceedings of the IEEE Workshop on Multimedia Signal Processing, Siena, Italy, September 2004, pp. 87-90.

[184] K. P. Subbalakshmi and Q. Chen, "Joint source-channel decoding for MPEG-4 coded video over wireless channels," in Proceeding of the IASTED International Conference on Wireless and Optical Communications, Banff National Park, Canada, July 2002, pp. 617-622.

[185] Q. Chen and K. P. Subbalakshmi, "Trellis decoding for MPEG-4 streams over wireless channels," in Proceedings of SPIE Electronic Imaging: Image and Video Communications and Processing, Santa Clara, CA, USA, January 2003, pp. 810-819.

[186] X. F. Ma and W. E. Lynch, "Iterative joint source-channel decoding using turbo codes for MPEG-4 video transmission," in Proceedings of the IEEE International Conference on Acoustics, Speech, and Signal Processing, vol. 4, Montreal, Quebec, Canada, May 2004, pp. 657-660.

[187] Q. Chen and K. P. Subbalakshmi, "Joint source-channel decoding for MPEG-4 video transmission over wireless channels," IEEE Journal on Selected Areas in Communications, vol. 21, no. 10, pp. 1780-1789, December 2003.

[188] Y. Wang and S. Yu, "Joint source-channel decoding for H.264 coded video stream," IEEE Transactions on Consumer Electronics, vol. 51, no. 4, pp. 1273-1276, Novemeber 2005.

[189] Q. Chen and K. P. Subbalakshmi, "An integrated joint source-channel decoder for MPEG-4 coded video," IEEE Vehicular Technology Conference, vol. 1, pp. 347-351, October 2003.

[190] K. Lakovic and J. Villasenor, "Combining variable length codes and turbo codes," in Proceedings of the IEEE Vehicular Technology Conference, vol. 4, Birmingham, AL, USA, May 2002, pp. $1719-1723$. 
[191] M. Grangetto, B. Scanavino, and G. Olmo, "Joint source-channel iterative decoding of arithmetic codes," in Proceedings of the IEEE International Conference on Communications, vol. 2, Paris, France, June 2004, pp. 886-890.

[192] H. Nguyen and P. Duhamel, "Iterative joint source-channel decoding of variable length encoded video sequences exploiting source semantics," in Proceedings of the International Conference on Image Processing, vol. 5, Singapore, October 2004, pp. 3221-3224.

[193] H. Xiao and B. Vucetic, "Channel optimized vector quantization with soft input decoding," in Proceedings of the International Symposium on Signal Processing and Its Applications, vol. 2, Gold Coast, Queensland, Australia, August 1996, pp. 501-504.

[194] D. J. Vaisey and A. Gersho, "Variable block-size image coding," in Proceedings of the IEEE International Conference on Acoustics, Speech, and Signal Processing, vol. 12, Dallas, TX, USA, April 1987, pp. 1051-1054.

[195] A. Makur and K. P. Subbalakshmi, "Variable dimension VQ encoding and codebook design," IEEE Transactions on Communications, vol. 45, no. 8, pp. 897-899, August 1997.

[196] S. X. Ng and L. Hanzo, "Space-time IQ-interleaved TCM and TTCM for AWGN and Rayleigh fading channels," Electronics Letters, vol. 38, no. 24, pp. 1553-1555, 2002.

[197] L. Hanzo, T. H. Liew, and B. L. Yeap, Turbo Coding, Turbo Equalisation and Space Time Coding for Transmission over Wireless Channels. Chichester, UK: Wiley, 2002.

[198] R. C. Bose and D. K. Ray-Chaudhuri, "On a class of error-correcting binary group codes," Information and Control, vol. 3, pp. 68-79, March 1960.

[199] A. Hocquenghem, "Codes correcteurs d'erreurs," Chiffres, vol. 2, pp. 147-156, September 1959.

[200] B. Masnick and J. Wolf, "On linear unequal error protection codes," IEEE Transactions on Information Theory, vol. 13, no. 4, pp. 600-607, October 1967.

[201] M. Rosenblatt, "A central limit theorem and a strong mixing condition," Proceedings of the National Academy of Sciences, no. 42, pp. 43-47, 1956.

[202] D. E. Goldberg, Genetic Algorithms in Search, Optimization and Machine Learning. AddisonWesley, 1989.

[203] G. Golub and C. van Loan, Matrix Computations. Baltimore: Johns Hopkins University Press, 1996.

[204] F. Gray, US Patent 2632 058, March, 1953.

[205] S. ten Brink, "Code characteristic matching for iterative decoding of serially concatenated codes," Annals of Telecommunications, vol. 56, no. 7-8, pp. 394-408, July-August 2001.

[206] A. Chindapol and J. A. Ritcey, "Design, analysis, and performance evaluation for BICM-ID with square QAM constellations in Rayleigh fading channels," IEEE Journal on Selected Areas in Communications, vol. 19, no. 5, pp. 944-957, May 2001. 
[207] J. Tan and G. L. Stuber, "Analysis and design of interleaver mappings for iteratively decoded BICM," in Proceedings of the IEEE International Conference on Communications, vol. 3, New York, NY, USA, April 2002, pp. 1403-1407.

[208] F. Schreckenbach, N. Görtz, J. Hagenauer, and G. Bauch, "Optimized symbol mappings for bit-interleaved coded modulation with iterative decoding," in Proceedings of the IEEE Global Telecommunications Conference, vol. 6, San Francisco, CA, USA, December 2003, pp. 33163320. 


\title{
Glossary
}

\author{
ACS Add, Compare and Select \\ ALU Arithmetic and Logic Unit \\ APP A Posteriori Probability \\ AWGN Additive White Gaussian Noise \\ $\mathrm{BCH} \quad$ Bose-Chaudhuri-Hocquenghem \\ BCJR Bahl-Cocke-Jelinek-Raviv \\ BEC Binary Erasure Channel \\ BER Bit Error Ratio \\ BICM Bit-Interleaved Coded Modulation \\ BPSK Binary Phase Shift Keying \\ CABAC Context Adaptive Binary Arithmetic Coding \\ CAVLC Context Adaptive Variable Length Coding \\ CC Convolutional Coding \\ COVQ Channel-Optimised Vector Quantisation \\ DCMC Discrete-input Continuous-output Modulated Channel \\ DCT Discrete Cosine Transform \\ EWVLC Even Weight Variable Length Coding \\ EXIT EXtrinsic Information Transfer \\ FD Frame Difference \\ GA Genetic Algorithm
}




\begin{tabular}{|c|c|}
\hline HA & Heuristic Algorithm \\
\hline HISO & Hard-In Hard-Out \\
\hline HMM & Hidden Markov Model \\
\hline IIR & Infinite Impulse Response \\
\hline IQ & In-phase Quadrature-phase \\
\hline $\mathrm{IrCC}$ & Irregular Convolutional Coding \\
\hline IrLDPC & Irregular Low Density Parity Check \\
\hline IrURC & Irregular Unity Rate Coding \\
\hline IrVLC & Irregular Variable Length Coding \\
\hline ISI & InterSymbol Interference \\
\hline IV-FD & Integer-Valued Free Distance \\
\hline LBG & Linde-Buzo-Gray \\
\hline LFSR & Linear Feedback Shift Register \\
\hline LLR & Logarithmic Likelihood-Ratio \\
\hline MAP & Maximum A posteriori Probability \\
\hline MB & Macro-Block \\
\hline MC & Motion Compensation \\
\hline MIMO & Multiple-In Multiple-Out \\
\hline ML & Maximum Likelihood \\
\hline MMSE & Minimum Mean-Squared-Error \\
\hline MPEG & Motion Picture Experts Group \\
\hline MSEW & Maximum Squared Euclidean Weight \\
\hline MSP & Modified Set Partitioning \\
\hline MUD & Multi-User Detection \\
\hline PDF & Probability Distribution Function \\
\hline PSK & Phase Shift Keying \\
\hline PSNR & Peak Signal to Noise Ratio \\
\hline QAM & Quadrature Amplitude Modulation \\
\hline QCIF & Quarter Common Intermediate Format \\
\hline RV-FDM & Real-Valued Free Distance Metric \\
\hline RVLC & Reversible Variable Length Coding \\
\hline SER & Symbol Error Ratio \\
\hline
\end{tabular}




$\begin{array}{ll}\text { SIHO } & \text { Soft-In Hard-Out } \\ \text { SISO } & \text { Soft-In Soft-Out } \\ \text { SNR } & \text { Signal to Noise Ratio } \\ \text { SOBIT } & \text { SOft BIT } \\ \text { SOVA } & \text { Soft Output Viterbi Algorithm } \\ \text { SP } & \text { Set Partitioning } \\ \text { SQNR } & \text { Signal to Quantisation Noise Ratio } \\ & \\ \text { TCM } & \text { Trellis Coded Modulation } \\ \text { TCQ } & \text { Trellis Coded Quantisation } \\ & \\ \text { UEP } & \text { Unequal Error Protection } \\ \text { URC } & \text { Unity Rate Coding } \\ & \\ \text { VB } & \text { Video Block } \\ \text { VDVQ } & \text { Variable Dimension Vector Quantisation } \\ \text { VLC } & \text { Variable Length Coding } \\ \text { VLEC } & \text { Variable Length Error Correction } \\ \text { VQ } & \text { Vector Quantisation }\end{array}$




\section{Index}

A

Additive white Gaussian noise (AWGN)...20, 23

Additive white Gaussian noise channel ...... 19

Aging . . . . . . . . . . . . . . 20

AR model properties . . . . . . . . . . . . 42

Autoregressive model . . . . . . . . . . . 41-42

Average conveyed mutual information per symbol

61

Average error entropy

. 62

Average information loss . . . . . . . . . 62

Average joint information . . . . . . . . . 60

Average loss of information per symbol.....663

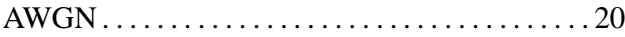

AWGN channel . . . . . . . . . . . . . . 20-23

B

Bahl-Cocke-Jelinek-Raviv (BCJR) algorithm 102 ,

144, 161, 198, 242, 286

Bayes' rule

. 49-52

Binary Phase Shift Keying (BPSK) . . . . 201, 284

Binary symmetric channel example

$.47-49$

C

Capacity of continuous channels

69-77

Capacity of discrete channels

Channel

. 19

channel capacity

84, 214, 252

$E_{b} / N_{0}$ bound

$148,165,216,254$

loss

$188,252,284$

channel coding
Channel coding theorem $\ldots \ldots \ldots \ldots \ldots \ldots 66$

Channel encoder ................. 18

Channel impairments . . . . . . . . . . . 17

channel-optimised source coding . . . . . . 85

Coaxial cable ................... 20

code constraint ........93, 138, 140, 155, 186

Coding efficiency ....................29

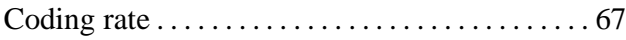

coding rate $\mathbf{8 9}, 146, \mathbf{1 5 5}, 180,186,237,273,278$, 281

Communications channels . ............20

Communications system design considerations 17 computational complexity ....105, 152, 161, 178, $218,228,238,265,279,280$

Cumulative density function . . . . . . . . 17

cutoff rate ......................84

E

effective throughput........148, 165, 214, 252

Entropy ................. 25-28

entropy .......84, 88, 146, 155, 186, 187, 240

Entropy of analogue sources ........... 70

Entropy of sources exhibiting memory . . . 35-37

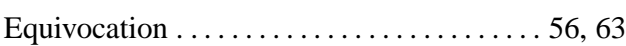

Error entropy ................... 57

Error entropy via imperfect channels . . . . 57-63

EXtrinsic Information Transfer (EXIT)

chart115, 116, 124, 149, 167, 173, 194, 208, $219,248,250,251,258,272,276$ 
decoding trajectory . 116, 117, 149, 172, $174,225,263,277$

matching... 171, 213, 237, 238, 252, 269 tunnel 118, 148, 169, 171, 172, 214, 225 , $252,261,263,270$

function. .112, 148, 166, 171, 185, 190, 234

\section{F}

First-order Markov model. $42-44$

Four-state Markov model for a 2-bit quantizer4041

frame differencing. ..130 free distance

98, 186

lower bound

99, 146, 163, 187

metric

189, 244,274

Free-space

.21

\section{G}

Gaussian

23

Gaussian noise...................20-23

Generating model sources..............41-44

Genetic Algorithm (GA) . 194, 203, 244, 256, 273

Group delay

20

\section{$\mathbf{H}$}

hard information.

100, 127, 163, 191

Huffman coding ... $31-35,90,137,171$

codebook

89,160

decoding

.. 9

Ideal communications system ......... 76-77

Information loss .................... 55

Information loss via imperfect channels . . 55-57

Information of a source ..............23-25

Information properties ................. 24

Information sources .................... 16

Information theory ............... 16-79

inter-frame redundancy ................. 130

Interleaver ......................... 19

interleaver109, 118, 133, 161, 175, 201, 222, 242,

260,277 intra-frame redundancy

130

Irregular Convolutional Coding (IrCC) . 123, 171

irregular trellis ........139, 140, 142, 143, 269

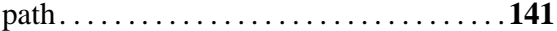

state $\ldots \ldots \ldots \ldots \ldots \ldots \ldots \ldots \ldots \ldots 14$

transition ....................... 140

Irregular Unity Rate Coding (IrURC) . . 240, 275

Irregular Variable Length Coding (IrVLC) . . . 83, $128,157,198,240$

Issues in information theory . . . . . . . . . . 16-20

iterative decoding . . . . 107, 133, 161, 201, 242

convergence. . 111, 147, 165, 179, 213, 251, 261,283

\section{$\mathbf{J}$}

Joint information . . . . . . . . . . . . . . . . 59 joint source and channel coding85, 129, 158, 200, 240, 268

$\mathbf{L}$

latency .................... 147, 278

Linde-Buzo-Gray (LBG) algorithm . . . . . . 137

Log-MAP algorithm . . . . 146, 161, 200, 220, 238

Logarithmic Likelihood Ratio (LLR) . . 101, 144, $161,191,201,242,263$

lossless compression. ...............130

lossy compression . . . . . . . . . . . . . . 130, 159

$\mathbf{M}$

macro-block ..................... 135

Markov model ..................... 35

Maximum entropy of a binary source . . . . 26-27

Maximum entropy of a $q$-ary source . . . . 27-28

Microwave channels .................20

Modulator ....................... 19

Mutual information . . . . . . . . . . 52-53

Mutual information example ........ 53-55

\section{$\mathbf{N}$}

$N$-state Markov model for discrete sources exhibiting memory ......... 36-37 
Noise - man-made

Noise - natural . .

21

21

$\mathbf{O}$

Optical fiber

20

\section{$\mathbf{P}$}

Peak Signal to Noise Ratio (PSNR) ... 150

Power spectral density

Practical evaluation of the Shannon-Hartley law 73-76

Practical Gaussian channels $20-21$

prefix condition ... 89

Probability density function

Q

Quadrature Amplitude Modulation (QAM) .. 147, 165,242

quantisation

87, $158,202,240$

Quantization

..16

\section{$\mathbf{R}$}

Rayleigh fading channel . 147, 158, 201, 240, 242

redundancy .............8. 83, 139, 155, 186

residual redundancy ................. 85

Reversible Variable Length Coding (RVLC) .. 91, 128,155

codebook. . .

$89, \mathbf{1 3 6}, 137$

decoding ...

92,143

encoding ... $138, \mathbf{1 4 2}$

RLC efficiency . 45-47

Run-length coding 44-47

Run-length coding principle. $.44-45$

$\mathbf{S}$

Sampling ... 16 sequence estimation . 95, 101, 104, 162, 171, 191, 202,243

serial concatenation 109, 132, 161, 198

Shannon's channel coding theorem

66-69

Shannon's message for wireless channels . . 77-79

Shannon-Fano coding

29-31, 89
Shannon-Hartley law .................. 73

side information ... 160, 162, 165, 200, 223, 241 ,

260, 261, 282

Signal-to-noise ratio $\ldots \ldots \ldots \ldots \ldots \ldots \ldots 17$

Skin effect.........................20

soft information...............100, 145, 163 a posteriori...108, 133, 145, 161, 171, 201, 243

a priori.......108, 133, 144, 161, 201, 243 extrinsic.........108, 133, 161, 201, 242 mutual information ..... 148, 166, 193, 249 Soft-In Soft-Out (SISO) decoding . 102, 133, 161, 201, 242

source and channel coding separation theorem $\mathbf{8 5}$, 126, 268

Source coding . . . . . . . . . . . 28-35

source coding .................84

Source encoder .............. 18, 28

Speech systems ................. 77

suffix condition $\ldots \ldots \ldots \ldots \ldots \ldots \ldots \ldots \ldots$

\section{$\mathbf{T}$}

T-BCJR algorithm ................ 146

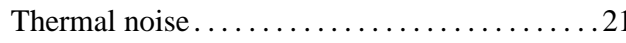

Time Division Multiple Access (TDMA) ... . 17

Transducer...................... 17

Transfer function $\ldots \ldots \ldots \ldots \ldots \ldots \ldots \ldots$

Transmission via discrete channels . . . . . . 47-63

Trellis Coded Modulation (TCM) . . . . . 132, 157

turbo code .................. 107

Turbo principle $\ldots \ldots \ldots \ldots \ldots \ldots \ldots \ldots \ldots$

Twisted pairs...................... 20

Two-state Markov model example . . . . . . 38-40

Two-state Markov model for discrete sources exhibiting memory ......... 35-36

$\mathbf{U}$

Unequal Error Protection (UEP) . . . 86, 156, 282

Unity Rate Coding (URC) . . . . . . . . . 188, 245 
V

Variable Dimension Vector Quantisation (VDVQ)

132

codebook ................. 136, 137

decoding .................... 143

encoding.................138, 142

Variable Length Coding (VLC) .........83, 157

average codeword length . .... 89, 155, 186

tree ........................90

Variable Length Error Correction (VLEC) coding 93, 155, 185, 244

bit-based trellis . . 93, 94, 161, 189, 198, 242

codebook . 89, 193, 204, 206, 246, 247, 257

decoding .....................96

symbol-based trellis .........96, 97, 161

Vector Quantisation (VQ) ............... 88

video

block .......................... 134

coding ....................... 127

compression ................... 129

information.................... 127

Viterbi algorithm ................. 95, 142

W

Waveguides.........................20 